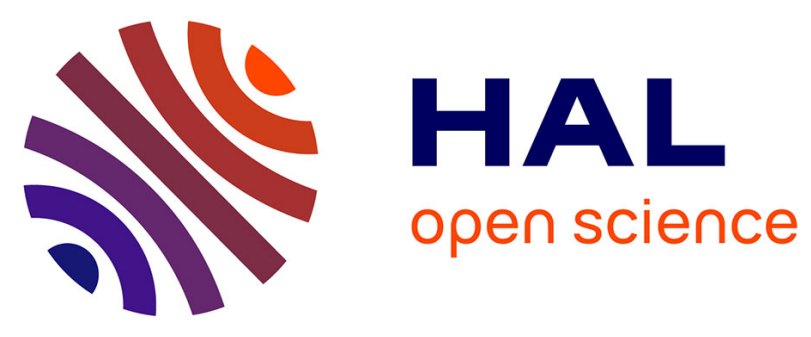

\title{
Échanges et consommation
}

Michel Bonifay, Claude Raynaud, Frédéric Berthault, Stéphane Bien, Élise Boucharlat, Danièle Foy, Tomoo Mukai, Emmanuel Pellegrino, Jean-Pierre Pelletier, Dominique Pieri, et al.

\section{- To cite this version:}

Michel Bonifay, Claude Raynaud, Frédéric Berthault, Stéphane Bien, Élise Boucharlat, et al.. Échanges et consommation. Gallia - Archéologie de la France antique, 2007, Antiquité tardive, haut Moyen Âge et premiers temps chrétiens en Gaule méridionale (seconde partie) : monde rural, échanges et consommation, 64, pp.93-161. 10.3406/galia.2007.3308 . hal-01911700

\section{HAL Id: hal-01911700 https://hal.science/hal-01911700}

Submitted on 29 Jan 2020

HAL is a multi-disciplinary open access archive for the deposit and dissemination of scientific research documents, whether they are published or not. The documents may come from teaching and research institutions in France or abroad, or from public or private research centers.
L'archive ouverte pluridisciplinaire HAL, est destinée au dépôt et à la diffusion de documents scientifiques de niveau recherche, publiés ou non, émanant des établissements d'enseignement et de recherche français ou étrangers, des laboratoires publics ou privés.

\section{(이)(\$)}

Distributed under a Creative Commons Attribution - NonCommercial - NoDerivatives| 4.0 


\title{
ÉCHANGES ET CONSOMMATION
}

\author{
Michel BONIFAY et Claude RAYNAUD
}

\author{
avec les contributions de Frédéric BERTHAULT, Stéphane BIEN, \\ Élise BOUCHARlat, Danièle FoY, Tomoo MUKaI, Emmanuel PELlegrino, \\ Jean-Pierre PELletiER, Dominique PIERI, Jean PITON, Yves RIGOIR, \\ Tony SILVINO, Jean-Christophe TRÉGLIA
}

Mots-clés. Amphore, vaisselle méditerranéenne, vaisselle commune, verrerie, datation, échanges.

Résumé. Au début des années 1980, lorsque les recherches sur l’Antiquité tardive commencèrent à se développer, le problème de datation des vestiges matériels constituait une tâche incontournable, conditionnant tout avancement des études. En Provence et Languedoc, ce fut l'ambition du groupe CATHMA (Céramique de l'Antiquité tardive et du haut Moyen Âge), à l'actif duquel on compte une quinzaine d'études consacrées à l'élaboration d'un cadre de référence typochronologique. Cette activité accompagnait l'essor des chantiers de fouille, tant en ville qu'à la campagne, se nourrissait des découvertes les plus récentes et contribuait en retour à l'affinement des cadres de datation. Livrant une documentation stratigraphique, ces chantiers marquèrent une étape cruciale de cet essor. Un premier essai de Dictionnaire des Céramiques antiques donnait une large place à ces productions tardo-antiques du Midi ainsi qu'aux importations méditerranéennes mais l’ouvrage, incomplet et désormais épuisé, devra être réédité avec de substantielles mises à jour, imposées par l'affinement régulier des connaissances. En Rhône-Alpes aussi, une entreprise collective permit d'élaborer un cadre de référence chronotypologique, tandis que plus récemment et sur une plus grande aire géographique se développèrent plusieurs enquêtes sur le mobilier de parure. Ces recherches métropolitaines trouvèrent par la suite leurs prolongements dans plusieurs régions méditerranéennes, de la Tunisie au Proche-Orient où de nombreux ateliers, d'amphore, de vaisselle ou de verrerie, furent localisés ou revisités à la faveur des nouvelles techniques d'étude.

Ainsi peut-on désormais proposer des datations allant parfois jusqu'au demi-siècle, voire même au quart de siècle près, tout au moins jusqu'au $V I^{e}$ s. Les repères faiblissent ensuite et il reste beaucoup à faire, notamment pour étayer et préciser encore de nombreux éléments grâce à la systématisation des dates par radiocarbone. Cet affinement des critères chronologiques a marqué une étape décisive pour sortir les études des interprétations approximatives. Au-delà des raffinements chronologiques autorisés par les études céramologiques, il s'agissait, bien plus fondamentalement, de sinterroger sur la diffusion des importations lointaines autant que des productions régionales, d'en suivre le flux et le reflux. Ainsi apparaît peu à peu une histoire des échanges qui échappe aux présupposés et aux stéréotypes d'une économie en repli à la fin de l'Antiquité.

Keywords. Amphora, Mediterranean vessels, coarse pottery, glass, date, trade.

Abstract. With the expansion of research on Late Antiquity in the early 80', the question of the datation of finds was a task that could not be escaped, for the advancement of these studies. In Provence and Languedoc, on the credit of the CATHMA programme (Céramique de l'Antiquité tardive et du haut Moyen Âge) which had this ambition, about fifteen reports have been devoted to the finalization of a typochronological reference pattern. In connection with the development of urban excavations, and as well in the countryside, this activity could use new discoveries and in turn help to work out a fine dating pattern. The stratigraphical data given by these excavations constituted a decisive step. A preliminary Dictionnaire des Céramiques antiques gave a large place to these Late southern productions and to Mediterranean imports. The book is not complete and now out-of-print; it should be published again and reactualized with important new evidence. A collective project has been working also in Rhône-Alpes on a chronological typopological reference system and more recently several surveys, on a larger area, were carried out on adornments. This research had effects in several Mediterranean areas. From Tunisia to the Near East amphorae and pottery fabrics and glass workshops have been located or re-examined with the help of new methods of study.

Consequently, from now on, it is possible to propose fine datations up to 50 years, even within a quarter of a century, at least until the $6^{\text {th }}$ century. Markers get poor afterwards and there is still much to do, especially to confirm and determine a number of points, due to systematic radiocarbone datation. This improvement of chronological criteras allow to escape approximative interpretations. Outside 
pottery studies bringing precise datation, more basically one had to examine the distribution of distant imports as of regional products and to follow their ebb and flow. Thus a history of trade is gradually emerging, avoiding presuppositions and stereotypes of a weakened economy in Late Antiquity.

Translation: Isabelle FAUDUET

Schlüsselwörter. Amphoren, mediterranes Geschirr, Gebrauchsgeschirr, Glas, Datierung, Warentausch.

Zusammenfassung. Als die Wissenschaftler zu Beginn der 80iger Jahre des 20. Jahrhunderts begannen sich vermehrt der Spätantike zu widmen, bestand eines der Hauptprobleme in der Datierung des Fundmaterials - Vorbedingung für jeglichen Fortschritt bei den Studien. In der Provence und im Languedoc machte die Studiengruppe CATHMA (Céramique de l'Antiquité tardive et du haut Moyen Âge) sich dies zur Aufgabe. Sie allein verbucht für sich an die fünfzehn Untersuchungen, die die Ausarbeitung eines typochronologischen Referenzrahmens zum Ziel hatten. Die Studien wurden von dem Grabungsboom dieser Jahre, sowohl in den Städten als auch auf dem Land begleitet, sie basierten demzufolge auf den neuesten Entdeckungen und steuerten im Gegenzug zur Verfeinerung der chronologischen Rahmen bei. Diese stratigraphisch dokumentierten Grabungen trugen wesentlich zu dem Aufschwung bei. Eine erste Auflage des Dictionnaire des Céramiques antiques räumte den spätantiken Keramikformen Südfrankreichs und den mediterranen Importwaren großen Raum ein, doch das Werk - unvollständig und inzwischen vergriffen - muss auf den neuesten Stand gebracht und neuaufgelegt werden, da die Erkenntnisse seitdem fortwährend aktualisiert wurden. Auch in der Region Rhône-Alpes konnte von einer Arbeitsgruppe ein typochronologischer Rahmen erarbeitet werden. Darüber hinaus wurden vor kürzerem und in einem weitergefassten geographischen Rahmen mehrere Untersuchungen zum Thema Schmuck durchgeführt. Diese Untersuchungen in Frankreich selbst wurden durch Studien in mehreren Regionen des Mittelmeerraumes von Tunesien bis in den Vorderen Orient ergänzt, wo zahlreiche Werkstätten, in denen Amphoren, Geschirr oder Glas hergestellt worden war, lokalisiert oder unter Berücksichtigung moderner Untersuchungstechniken erneut untersucht wurden.

So können wir heute, zumindest bis zum 6. Jahrhundert, Datierungen mit einer Spanne von nur 50 oder sogar nur 25 Jahren vorschlagen. Danach werden die Bezugspunkte seltener und es steht noch viel Arbeit an, insbesondere um durch den systematischen Einsatz der Radiokarbondatierung zahlreiche Elemente zu untermauern und genauer zu bestimmen. Diese Verfeinerung der chronologischen Kriterien kennzeichnete eine entscheidende Etappe, die es den Studien ermöglichte die Phase der approximativen Interpretationen hinter sich zu lassen. Über die chronologischen Feinheiten hinaus, die die keramologischen Studien ermöglichten, ging es im Grunde viel mehr darum, sich die Frage nach der Verbreitung der Importe aus fernen Regionen und der regionalen Produkte zu stellen, und deren Vordringen und Zurückweichen zu verfolgen. So wird nach und nach eine Geschichte des Warentauschs erkennbar, die von den vorgefassten Meinungen und Verallgemeinerungen von einer rückläufigen Wirtschaft am Ende des Altertums nicht berücksichtigt wird.

Übersetzung: Isa ODENHARDT-DONVEZ

Dresser un bilan des connaissances sur les céramiques de l'Antiquité tardive suppose en préalable de définir des bornes chronologiques. Aucune césure ne s'impose en regard de l'évolution des productions, les unes déclinant lentement tandis que d'autres, appelées à les remplacer, s'imposent graduellement. Amphores, vaisselle fine et vaisselle commune obéissent chacune à un rythme singulier, l'ensemble des transitions tissant une trame complexe.

Ainsi par exemple, si la vaisselle fine des $\mathrm{IV}^{\mathrm{e}}$ et $\mathrm{V}^{\mathrm{e}} \mathrm{s}$. reste dominée par trois principales productions, sigillées africaines $\mathrm{C}$ et $\mathrm{D}$, et Luisante rhodanienne, la première se trouve attestée vers le premier quart du $\mathrm{III}^{\mathrm{e}}$ s., tandis que la seconde s'affirme seulement au siècle suivant, la troisième émergeant, quant à elle, dans l'entre-deux, quelque part dans la seconde moitié du $\mathrm{III}^{\mathrm{e}} \mathrm{s}$.

La même progression caractérise la vaisselle commune, qui voit sa production se diversifier et sa diffusion étendre son rayon, quelque part au $\mathrm{III}^{\mathrm{e}}$ s., lorsque se diffusent vers le sud les productions micacées de la vallée du Rhône, ou bien celle des poteries kaolinitiques au $\mathrm{IV}^{\mathrm{e}} \mathrm{s}$.

S'il existe des traits caractéristiques de la vaisselle tardoantique, rien dans sa morphologie ni dans sa typologie ne laisse alors envisager des usages nouveaux, tandis que se perpétuent formes et fonctions connues depuis le début de la période gallo-romaine.

On ne s'étonnera donc pas de trouver notre enquête bornée de la façon la plus lâche qui soit, depuis le milieu du $\mathrm{III}^{\mathrm{e}} \mathrm{s}$. jusqu'à la fin du VII ${ }^{\mathrm{e}} \mathrm{s}$., ces bornes n'ayant d'autre valeur que de convenance et que rien, ni rupture ni bouleversement, ne justifie un découpage que l'on aurait pu étendre dans un sens ou dans l'autre. Si les faciès céramiques de la seconde moitié du $\mathrm{III}^{\mathrm{e}} \mathrm{s}$. plongent de solides racines dans les traditions du Haut-Empire, il faudrait en revanche poursuivre un bon siècle, jusqu'à la fin du VIII ${ }^{\mathrm{e}}$ s., 
pour voir s'effectuer la " médiévalisation » de la céramique, comme cela est patent en Languedoc (CATHMA, 1993, p. 122-123), en Provence (Pelletier, 1997, p. 111-115) et en Lyonnais (Faure-Boucharlat dir., 2001). Ce processus est marqué par la disparition des vaisselles fines, d'abord celles produites en Gaule, Luisante et Dérivée-de-Sigillées Paléochrétiennes (DS.P.), puis par celles des importations en provenance d'Afrique du Nord ou du Proche-Orient. Si certaines poteries orientales parviennent encore, au début du VIII ${ }^{\mathrm{e}}$ s., sur certains sites portuaires, essentiellement à Marseille, l'équipement domestique est désormais, pour de longs siècles, composé de céramiques communes siliceuses dont le répertoire se limite aux pots et ollae, ustensiles culinaires et de stockage. Très progressivement, les VI ${ }^{\mathrm{e}}$ et VII ${ }^{\mathrm{e}} \mathrm{s}$. voient s'opérer la disparition de la tradition céramique de l'Antiquité, en particulier celle qui reposait sur l'utilisation d'un mobilier de service à pâte calcaire désormais absent des dépotoirs domestiques.

Un autre mouvement se dessine au même moment, celui d'un basculement de l'aire d'approvisionnement en produits lointains. Longtemps restreintes à quelques unités, une céramique d'Argonne, à Marseille (Bonifay et al. dir., 1998, p. 366), deux à Lyon (Ayala, 1998, p. 230), quelques pièces de métallescente en Arles, à Loupian (Rouquette, Pellecuer, 1976-1977) ou Rodez (Boudartchouk, Llech, 1993, p. 154), les importations septentrionales en Gaule méditerranéenne croissent à mesure que faiblissent les flux maritimes. Sans atteindre le volume ni la diversité des céramiques méditerranéennes qu'ils n'ont, du reste, jamais remplacé, les arrivages croissent de façon sensible dès la fin $\mathrm{du} \mathrm{VI}^{\mathrm{e}} \mathrm{s}$., avec pour seul objet actuellement identifié les productions à pâte bistre de la vallée de la Saône, dont la diffusion reste à préciser mais ne fait aucun doute jusqu'au littoral. Verra-t-on là un signe, ou en tout cas un indice, en faveur de la vieille thèse de Pirenne sur le déplacement du centre de gravité de l'économie de la Méditerranée vers l'Europe occidentale? Malgré leur accroissement, les données demeurent lacunaires et leur cartographie encore trop aléatoire pour que l'on tranche ce débat.

Vingt ans après la première synthèse concernant la Gaule méditerranéenne (CATHMA, 1986), l'avancement des recherches autorise d'amples mises à jour et fait apparaître certains traits méconnus jusqu'à ces dernières années. Ces avancées restent néanmoins inégales, tant à l'égard des différentes productions qui ne bénéficient pas d'égale façon de ces progrès, soit à l'égard de la géographie des travaux qui connaît encore des zones d'ombre. Si l'on peut noter l'accroissement sensible des fouilles dans l'ensemble des régions concernées, ce développement reste essentiellement attaché à l'essor de l'archéologie préventive qui n'offre pas toujours un cadre propice à l'exploitation et à l'édition de la documentation, de sorte qu'un important décalage sépare les données potentiellement disponibles et les documents accessibles grâce aux publications. Ces dernières demeurent insuffisantes et concernent essentiellement les villes de Narbonne (Solier dir., 1991) et de Marseille (Bonifay et al. dir., 1998), ainsi que la région lyonnaise (Faure-Boucharlat dir., 2001), auxquelles ont été consacrées d'importantes monographies, de même que l'atelier savoyard de Portout (Pernon, Pernon dir., 1990). Des études plus ponctuelles, mais nombreuses, ont été réalisées en Languedoc, tant en ville (Monteil dir., 1993 ; Ginouvez dir., 1996-1997) qu'à la campagne (Garnier et al., 1995 ; Mauné, 1997-1998 ; Barberan, Pomarèdes, 1991 ; Térès, Rigoir, 2001), et en Provence (Pellegrino, 2001 ; Tréglia, 2004). C'est la même évolution que l'on observe en Rhône-Alpes, à Lyon (Ayala, 1998 ; Horry, 2000) et dans sa région (Ayala, 1996 ; Laroche, Théolas, 2003 ; André, 2003), ainsi que dans la région du lac Léman où le travail pionnier sur Genève (Paunier, 1981) se trouve régulièrement nuancé par des bilans et des études monographiques (Haldimann, 1994 ; David-Elbiali, Paunier dir., 2002).

Ces mises à jour, qui ne citent que les études principales et qui pourraient être multipliées, éclairent une géographie des recherches privilégiant la Gaule du Sud-Est, notamment les provinces de Narbonnaise et de Viennoise, auxquelles on peut annexer la région lyonnaise qui est parcourue par les mêmes courants. Par contraste, le bilan apparaît moins fourni en ce qui concerne les provinces occidentales, l'Aquitaine et la Novempopulanie, où les études céramologiques demeurent peu nombreuses et essentiellement consacrées à l'interprétation de niveaux d'occupation ou de structures de production, notamment à Toulouse (Catalo et al., 1998) ou bien dans l'atelier de potier de Martinière, aux marges de l'Aquitaine (Robin, Chambon, 2002). Si les ateliers régionaux ont bénéficié de nombreuses découvertes (Dieulafait et al., 1996), seules les céramiques fines DS.P. ont fait l'objet d'études suivies, tandis qu'un bilan rassemble des premiers éléments de synthèse sur les importations d'amphores (Amiel, Berthault, 1996). Même constat pour le Massif central où de premiers jalons ont été posés, à Rodez (Boudartchouk, Llech, 1993 ; Bourgeois, 1993), et dans sa région (Boudartchouk, 1993), mais où les centres producteurs, les aires de diffusion et les faciès demeurent méconnus. On ne saurait trop encourager le déploiement de recherches dans ces régions afin de combler le fossé qui sépare le littoral méditerranéen de la Gaule centrale. 
Le bilan sera donc limité, autant par l'inégal avancement des études que par la nécessité de se fier à une documentation bibliographique qui ne permet pas d'établir des similitudes rigoureuses entre les différentes productions. Ce problème concerne particulièrement les céramiques communes dont il est difficile d'établir des faciès et des aires de diffusion, entreprise qui reste à promouvoir par l'analyse physico-chimique ainsi que par des enquêtes systématiques dans les dépôts de fouille, qui supposent un travail collectif de longue haleine. Les pages qui suivent proposent un état des lieux et une série d'hypothèses d'attente.

C. R.

\section{L'APPORT DES AMPHORES À LA CONNAISSANCE DES ÉCHANGES}

Les premières études quantitatives sur les amphores importées en Gaule du Sud durant l'Antiquité tardive avaient bouleversé un certain nombre d'idées reçues sur le commerce (ou plutôt la crise du commerce) de l'Antiquité tardive. Elles faisaient échos aux recherches menées au même moment dans d'autres grandes cités de Méditerranée occidentale : Carthage, Rome, Porto Torres. Qu'en est-il vingt ans après? Les difficultés alors évoquées dans l'interprétation de cette documentation archéologique ont-elles été surmontées ? Connaît-on mieux l'origine et le contenu des amphores de l'Antiquité tardive ? Dans quelle direction doit-on actualiser les tendances économiques mises en évidence en 1986 ?

\section{TOUT LE COMMERCE NE SE FAIT PAS EN AMPHORES}

Dans son introduction sur "Le monde des échanges", P.-A. Février avait bien souligné la difficulté de passer de l'étude des céramiques à l'approche économique (Février, Leyge dir., 1986, p. 166). N'est-il pas illusoire de vouloir reconstituer l'histoire du commerce à partir de la seule documentation céramique, même lorsque celle-ci provient de récipients - les amphores - qui ont servi à transporter des denrées de première nécessité ? Cette réflexion méthodologique est toujours d'actualité. Nous prendrons trois exemples.

\section{LE TRANSPORT DU VIN EN BARRIQUES}

On sait maintenant qu'il faut accorder sans doute une place importante aux tonneaux aussi bien pour le commerce du vin (Marlière, 2002) que celui des salaisons de poissons. Les exemples en sont assez rares en Gaule du Sud : on retiendra cependant la base de tonneau retrouvée dans l'épave Saint-Gervais 2 à Fos-sur-Mer, datée du VII ${ }^{\mathrm{e}} \mathrm{s}$. (Jézégou, 1998, p. 345). Le volume des denrées contenues dans les tonneaux, au premier rang desquelles figure peutêtre le vin de Narbonnaise à partir du IV ${ }^{\mathrm{e}} \mathrm{s}$. (Raynaud dir., 1990, p. 296), constitue donc une inconnue difficile à résoudre dans l'équation des échanges de la Gaule du Sud durant l'Antiquité tardive. L'huile, notamment celle produite localement, pouvait quant à elle être transportée dans des outres (Marlière, 2002).

\section{LE TRANSPORT DU BLÉ EN VRAC}

Autre inconnue de taille : le blé. Alors qu'il est notoire que l'approvisionnement en céréales est une préoccupation majeure des sociétés de l'Antiquité, on reste très mal informé sur ce commerce durant toute l'époque romaine, tout au moins du point de vue archéologique (on ne connaît aucune épave de navire de l'annone). Dans ce contexte, il est intéressant de relever que l'une des rares épaves de Méditerranée portant les traces d'un chargement de blé est tardive et a été découverte à Fos-sur-Mer (Saint-Gervais 2, voir Jézégou, 1998).

\section{RARES CORRESPONDANCES ENTRE LES TEXTES ET L'ARGHÉOLOGIE}

L'examen des textes apporte une preuve supplémentaire des difficultés d'utilisation des amphores pour parvenir à une histoire économique (arguments rappelés par Loseby, 1998, p. 218-220). Certes, il y a parfois correspondance directe entre les textes et la documentation céramique: le vin de Gaza, dont Grégoire de Tours vante les mérites, est effectivement bien attesté par les amphores LRA 4 largement diffusées sur le littoral et le long de la vallée du Rhône (Pieri, 2005). Cependant, on relève également de nombreux décalages, tel le bateau de la peste qui, en 588 , arrive à Marseille en provenance d'Espagne «avec sa cargaison habituelle» alors que les amphores hispaniques ont quasiment disparu des stratigraphies dès le milieu du $\mathrm{V}^{\mathrm{e}} \mathrm{s}$. (Bonifay et al. dir., 1998). En revanche, il n'est jamais question du commerce africain, pourtant le mieux attesté par les céramiques dans cette ville. De même, l'huile est l'une des marchandises le plus souvent citées (Pernoud, 1949, p. 121), alors que la plupart des amphores retrouvées dans le port de Marseille, poissées, n’ont pu en contenir. On pourrait multiplier ces exemples et citer également toutes les 
marchandises mentionnées par les textes mais qui laissent difficilement des traces archéologiques : épices, aromates, peaux et cuirs, papyrus mais aussi esclaves dont Marseille était l'un des marchés principaux (Loseby, 2000, p. 177).

\section{LES DIFFÉRENTES RÉGIONS REPRÉSENTÉES : NOUVELLES DONNÉES SUR LES ZONES DE PRODUCTION}

Toutefois, les progrès accomplis dans l'étude des amphores depuis 1986 sont notables, tout d'abord du point de vue des zones de production. On relèvera trois avancées dans ce domaine, particulièrement spectaculaires.

\section{L'ITALIE ET L'ESPAGNE}

Dans le tableau général sur les importations méditerranéennes à Marseille, Lyon, Arles et Narbonne, dressé en 1986 par F. Villedieu (Février, Leyge dir., 1986, p. 182-183), l'Italie brillait par son absence : il semblait ne plus y avoir aucune trace d'amphore en provenance de la péninsule depuis les décennies centrales $d u \mathrm{IV}^{\mathrm{e}} \mathrm{s}$. Certes, on aurait pu douter du silence de la céramique et supputer que le vin italien parvenait en Gaule dans des barriques mais, au cours des années 1990, l'Italie s'est vu finalement attribuer deux types d'amphores tardives. Il est particulièrement symptomatique qu'il ne s'agit pas de formes nouvelles, mais de formes déjà connues pour lesquelles les hypothèses de provenance ont été radicalement modifiées. Il en est ainsi, tout d'abord, des amphores Ostia IV, 167 (Carandini, Panella dir., 1968) ou Agora M254 (Robinson, 1959) (fig. 50, no 1) dont les variantes tardives sont abondantes au $\mathrm{IV}^{\mathrm{e}} \mathrm{s}$, et qui ont été longtemps considérées comme d'origine tripolitaine, en raison de leur abondance dans les dépôts de fouilles de Leptis Magna; des découvertes récentes ainsi que les analyses pétrographiques permettent aujourd'hui, de façon plus plausible, d'admettre une origine sicilienne. C'est également le cas des amphores Keay 52 (fig. 50, no 2), particulièrement fréquentes à Marseille au $\mathrm{V}^{\mathrm{e}} \mathrm{s}$., que l'on proposait, jusqu'à la fin des années 1980, d'attribuer à la Méditerranée orientale (Bonifay, Villedieu, 1989, p. 33). Or, au même moment (Arthur, 1989), des prospections d'ateliers permettaient de rectifier cette hypothèse: ces conteneurs avaient, en fait, été produits dans le Bruttium! Il est évident que de telles révisions ont considérablement modifié les interprétations des statistiques établies en 1986 à Marseille (Bonifay, 1986, p. 297 ; nouvelle interprétation : Loseby, 1992, p. 185, tabl. 2).
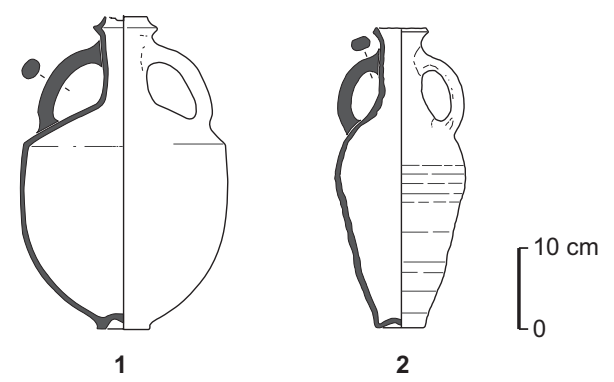

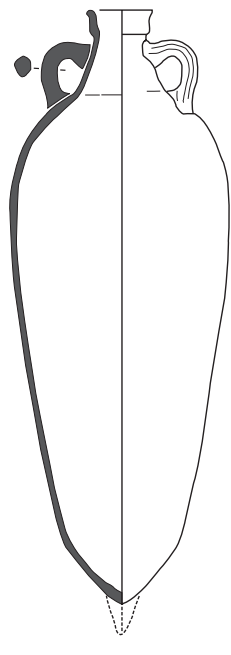

3

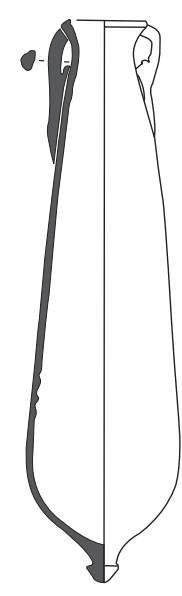

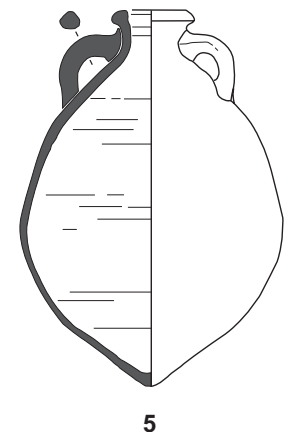

Fig. 50 - Amphores italiques : 1, type Agora M254, Saint-Tropez, épave de Pampelonne (d'après Lequément, 1976) ; 2, type Keay 52, Marseille, la Bourse (d'après Bonifay, 1986). Amphores hispaniques : 3, type Almagro 51a-b, Narbonne (d'après Belbenoit in Ginouvez dir., 1996-1997) ; 4, type Almagro 50, épave Port-Vendres I (d'après Chevalier, Santamaria, 1971) ; 5, type Dressel 23, Portout (d'après Pernon, Pernon dir., 1990) (DAO : M. Bonifay, CNRS).

\section{LA MÉDITERRANÉE ORIENTALE}

La présence massive des amphores orientales dans les grandes villes de Gaule méridionale, à partir du $\mathrm{V}^{\mathrm{e}} \mathrm{s}$., avait été la grande révélation des années 1980. On s'aperçoit aujourd'hui que ces documents alors insolites étaient « comme des invitations à la recherche, des pierres en attente » (Février, Leyge dir., 1986, p. 167). On en sait aujourd'hui beaucoup plus sur ces amphores grâce aux travaux conduits sur les lieux mêmes de production par des archéologues qui étaient préoccupés par la présence de ce mobilier en Méditerranée occidentale, que ce soit P. Reynolds après ses travaux en Espagne (Reynolds, 1995 ; voir aujourd'hui : Reynolds, 2005) ou D. Pieri à l'issue de sa 
thèse sur la diffusion des amphores orientales en Gaule et de son séjour à l'IFAPO de Beyrouth (Pieri, 2005). Le gain est à la fois d'ordre géographique - on s'aperçoit que ces amphores sont issues d'une multitude d'ateliers disséminés sur un vaste territoire et non pas seulement des quelques grands centres connus en 1986-, et d'ordre chronologique - puisque ces objets deviennent aujourd'hui de véritables critères de datation, presque aussi fiables que les sigillées (voir D. Pieri, notice 1, infra, p. 149-152).

\section{L'AFRIQUE}

Du point de vue de leurs productions céramiques, les provinces du diocèse d'Afrique étaient, en 1986, considérées au pire comme un tout, au mieux comme une sorte d'arrièrepays de la mégalopole et du port de Carthage. Il était très difficile, sauf pour quelques types particuliers (par exemple les types tripolitains majoritairement attribués à Leptis Magna et les amphores Dressel 30 supposées d'origine maurétanienne), d'établir des distinctions au sein du mobilier africain recueilli en Gaule méridionale (voir F. Berthault, notice 2, infra, p. 152-153), et notamment de reconnaître les parts respectives de la Zeugitane et de la Byzacène (fig. 51 et 52).

Ce sont tout d'abord les prospections du Sahel tunisien qui ont mis en évidence la densité des ateliers dans cette région et ce jusqu'à une date très tardive (Peacock et al., 1989 et 1990). Ceux de la périphérie de Sullecthum/Salakta, dont les productions sont facilement reconnaissables à leur pâte bicolore orange et grise, constellée de petites particules blanches (Capelli et al., 2006), continuent d'alimenter une bonne part des importations de Gaule méridionale au $\mathrm{IV}^{\mathrm{e}}$ s., comme le prouve la cargaison de l'épave Héliopolis I, au large de l'île du Levant (Joncheray, 1997 ; Bonifay et al., 2002). Après s'être déplacés de la périphérie urbaine vers des zones plus rurales, des ateliers plus tardifs, comme celui de Henchir ech-Chekaff, à $6 \mathrm{~km}$ de Salakta, sont probablement à l'origine de deux types d'amphores (types Keay 61C et Bonifay, 1986, fig. 12, n 55) fréquents à Marseille à la fin du VI ${ }^{\mathrm{e}}$ s. ou au début du VII ${ }^{\mathrm{e}}$ s. (Bonifay, Pieri, 1995, fig. 4). D’autres ateliers, repérés en Byzacène méridionale (Bonifay, 2004, p. 31), produisent des amphores (Keay 8B) principalement diffusées à l'époque vandale et dont on retrouve des exemples à Marseille (Bonifay, Pieri, 1995, fig. 2, $\mathrm{n}^{\text {os }} 13-14$ ) et à Nîmes (Manniez, 1993, fig. 147, no 83).

En Zeugitane, le cap Bon, notamment la région de Nabeul, est également une région très dynamique durant l'Antiquité tardive. Les amphores Africaine II C, bien

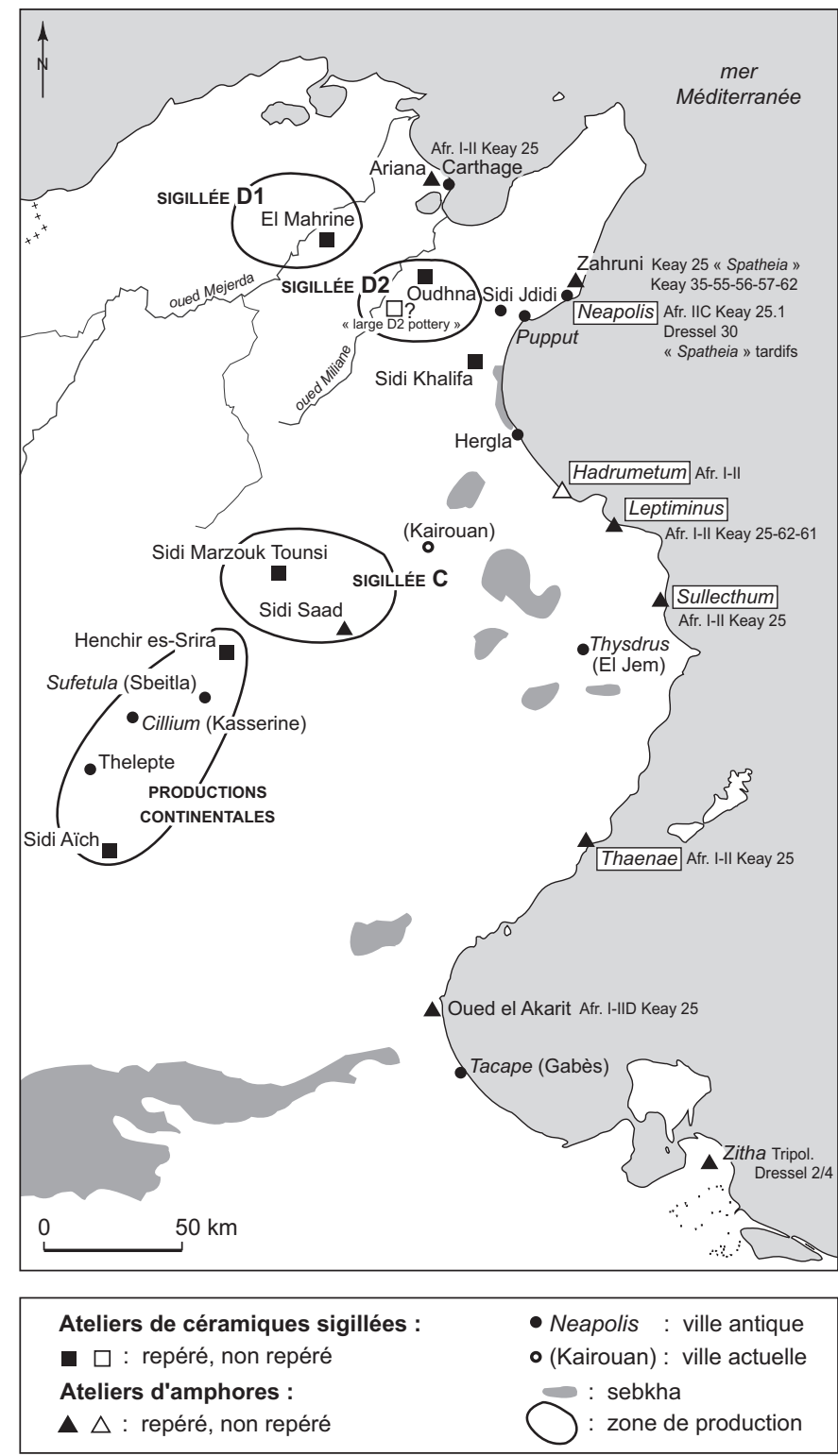

Fig. $\mathbf{5 1}$ - Carte des ateliers africains dont la diffusion est attestée en Gaule du Sud (DAO: M. Bonifay, CNRS).

attestées sur les ateliers de la périphérie de Nabeul (Ghalia et al., 2005), sont connes à Lyon dès la seconde moitié du III $^{\mathrm{e}}$ s. (voir T. Silvino, notice 3, infra, p. 153-155) et les amphores Keay 25 issues des mêmes ateliers composent une partie de la cargaison de l'épave de Pampelonne au IV ${ }^{\mathrm{e}} \mathrm{s}$. (Bonifay et al., 2002). Mais ces ateliers, notamment celui de Sidi Zahruni à Beni Khiar, reconnaissables à leur belle pâte orange vif avec des inclusions de quartz éolien et de grès quartzeux (ibid.), sont surtout actifs aux $\mathrm{V}^{\mathrm{e}}$ et $\mathrm{VI}^{\mathrm{e}}$ s., lorsque les types Keay 35, d'une part, et Keay 55, d'autre part, inondent les sites urbains et ruraux du Midi méditerranéen 


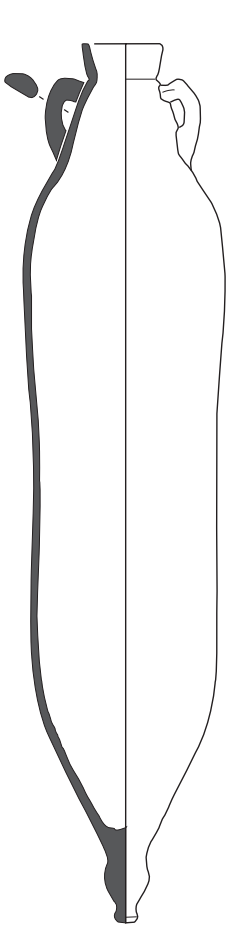

1
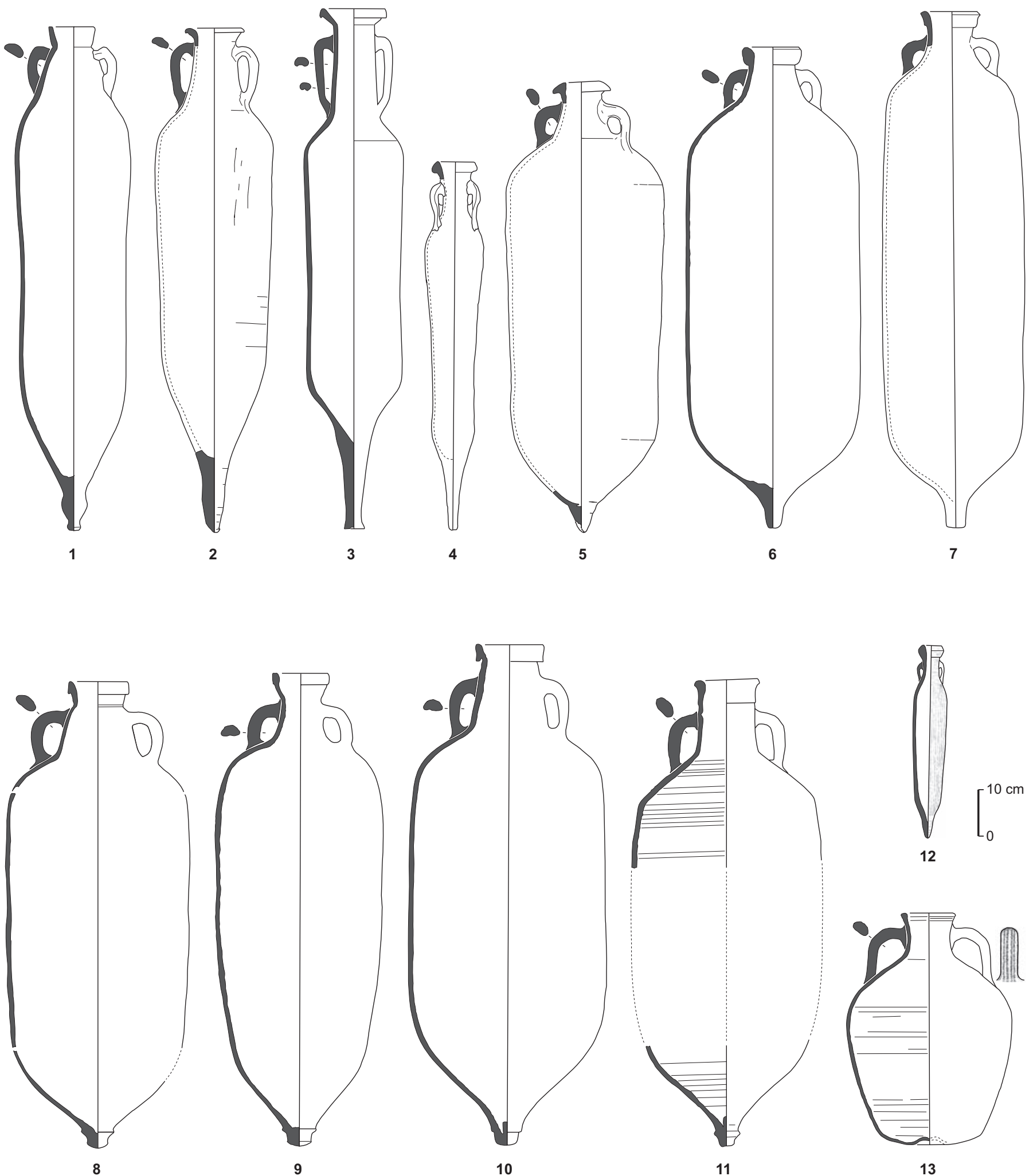

Fig. 52 - Principaux types d'amphores africaines attestés en Gaule du Sud : 1, type Keay 25.1, épave Héliopolis I (d'après Joncheray, 1997) ; 2, type Keay 25.3, musée d'Antibes (d'après Bonifay, 2004) ; 3, type Keay 25.2, épave Dramont E (d'après Joncheray, 1975) ; 4, «spatheion » 1, épave Dramont E (d'après Santamaria, 1995) ; 5, type Keay 35B, épave Dramont E (d'après Bonifay, 2004) ; 6, type Keay 57, golfe de Fossur-Mer (d'après Sciallano, Sibella, 1991) ; 7, type Keay 8B, baptistère d'Albenga, Italie (d'après Pallarès, 1987) ; 8, type Keay 62A, Camargue (d'après Tréglia, 2004) ; 9, type Keay 62 variante, Marseille, la Bourse (d'après Bonifay, 1986, fig. 12.55) ; 10, type Keay 61, Marseille, île de Pomègues (d'après Liou, 1975) ; 11, type Keay 8A, Finale Ligure (d'après Murialdo, 1988) ; 12, "spatheion » 3C, Les Angles, Saint-Étiennede-Candau (d'après Gagnière, 1965) ; 13, type Castrum Perti, Sant'Antonino di Perti (d'après Murialdo, 2001) (DAO : M. Bonifay, CNRS). 
(Ghalia et al., 2005). Deux épaves, celle du Dramont $E$ pour le deuxième quart $\mathrm{du} \mathrm{V}^{\mathrm{e}} \mathrm{s}$. (Santamaria, 1995) et celle de la Palud pour le deuxième quart $\mathrm{du} \mathrm{VI}^{\mathrm{e}} \mathrm{s}$. (Long, Volpe, 1998) sont le témoin de ces échanges (Bonifay et al., 2002).

Paradoxalement, l'ouest de la Zeugitane et la région de Carthage restent encore mal connus mais sont probablement à l'origine d'une bonne part des amphores Keay 25, 27 et 36 , fréquentes en Gaule méridionale au $\mathrm{IV}^{\mathrm{e}} \mathrm{s}$. et au début du $\mathrm{V}^{\mathrm{e}} \mathrm{s}$.

Enfin, les choses se compliquent pour les types africains dont on pensait qu'ils n'étaient pas d'origine tunisienne. On sait maintenant que la partie la plus occidentale de la province de Tripolitaine, autour de l'île de Jerba, en Tunisie, est sans doute à l'origine des amphores de type tripolitain qui possèdent une pâte fine, rosée, au lieu de la pâte classique bicolore rouge et grise à grosses inclusions blanches, qui est en fait caractéristique des productions de Leptis Magna. De même, il semble établi aujourd'hui que la Maurétanie césarienne, et notamment la cité de Tubusuctu, n'est plus le seul centre producteur des amphores Dressel 30 et de leurs variantes tardives, si fréquentes à Arles au IV ${ }^{\mathrm{e}}$ s., mais qu'il faut également compter avec des ateliers situés en Zeugitane et en Byzacène (Bonifay, 2004, p. 148). Parallèlement, il est vraisemblable, compte tenu de la diversité des pâtes, que toutes les formes classiques d'amphores africaines ne proviennent pas de Zeugitane ou de Byzacène et que des prospections menées en Algérie, lorsqu'elles seront à nouveau possibles, sont susceptibles de modifier grandement notre perception actuelle des importations africaines.

Il reste à souligner que la plupart des types connus au milieu des années 1980 ont vu leurs datations modifiées (comparer Keay, 1984 et Keay, 1998), sur la base, notamment, des stratigraphies du midi de la France (Bonifay, Pieri, 1995).

Enfin, s'il est un domaine où il reste encore beaucoup de progrès à faire, c'est celui du contenu des amphores importées en Gaule méridionale durant l'Antiquité tardive. Le contenu des amphores d'Italie du Sud et de la majorité des types orientaux ne paraît guère poser de problème : le vin semble en effet la solution la plus vraisemblable, alors même qu'à la fin des années 1980 il était encore de mise de retenir, au moins partiellement, l'hypothèse d'un contenu oléagineux pour les amphores LRA 1 (Bonifay, Villedieu, 1989 , p. 25). Il semble en revanche qu'on ait accordé une importance peut-être trop exclusive à l'huile en ce qui concerne les amphores africaines. Une enquête menée sur les exemplaires conservés dans les dépôts du Département des recherches archéologiques subaquatiques et sousmarines montre qu'une majorité d'entre eux sont poissés et n'ont pu, en conséquence, contenir de l'huile (Ben Lazreg et al., 1995, p. 125). Pour l'Antiquité tardive, il ne reste guère que les types Keay 59/8B, 35A et peut-être Keay 27 et 61C qui soient susceptibles d'être attribués au transport de l'huile (Bonifay, 2004, tabl. IV). Tous les autres présentent, soit de manière occasionnelle (Keay 27-36 et 55-57), soit de manière systématique (Keay 25 et $35 \mathrm{~B}$ ), des traces de poix. Le problème est de déterminer quel pouvait être le contenu des amphores africaines poissées : des salsamenta, comme les amphores Africaines II de Lyon (voir T. Silvino, notice 3, infra, p. 153-155) ? Du vin, comme les amphores Keay 25 de l'épave Héliopolis 1 (Formenti, Joncheray, 1995) ? Dans ce domaine, il y a beaucoup à attendre du développement des analyses (Garnier, 2007).

\section{NOUVELLES DONNÉES SUR LES FLUX COMMERCIAUX}

Les données dont nous disposons pour évaluer la part des différents flux commerciaux qui atteignent la Gaule du Sud au cours des $\mathrm{IV}^{\mathrm{e}}-\mathrm{VII}{ }^{\mathrm{e}} \mathrm{s}$. sont très lacunaires et inégalement réparties du point de vue chronologique et géographique. Aujourd'hui comme en 1986, quelques grandes villes seulement (Marseille, Arles, Lyon, Bordeaux) bénéficient de comptages précis. Mais, correspondant rarement aux mêmes tranches chronologiques, ces derniers sont, de fait, difficilement comparables entre eux. Les campagnes sont encore moins bien connues, à l'exception de quelques sites du Languedoc (par exemple Lunel-Viel, voir Raynaud dir., 1990) et de Provence (par exemple Eyguières, voir Pelletier et al., 2000). Quelques tendances apparaissent cependant, certaines confirmant les données de 1986, d'autres plus novatrices.

\section{LA DOMINATION DES AMPHORES AFRICAINES AU IV ${ }^{\mathrm{e}} \mathbf{S}$.}

Contrastant avec la période des II $^{\mathrm{e}}$-III ${ }^{\mathrm{e}}$ s., caractérisée à la fois par l'hégémonie du vin de Narbonnaise (quasimonopole des amphores gauloises, notamment en milieu rural, voir Raynaud dir., 1990, p. 295) et une ouverture modeste mais diversifiée aux denrées des autres provinces (principalement dans les grandes villes, par exemple à Marseille, voir Lang, 1998b, p. 93 et tabl. X), le $\mathrm{IV}^{\mathrm{e}} \mathrm{s}$. voit s'affirmer partout les produits africains (amphores Keay 25). Quels sont ces produits? Aux arguments en faveur du vin évoqués plus haut, l'archéologie rurale en 
Languedoc ajoute les siens en démontrant la coïncidence troublante (Pellecuer, 2000 ; voir cependant les remarques sur le tonneau plus haut) entre la disparition des amphores gauloises et les arrivages massifs d'amphores africaines cylindriques de moyennes dimensions (plus de $70 \%$ à Lunel-Viel au début du IV ${ }^{\mathrm{e}}$ s., voir Raynaud dir., 1990, p. 296). Cette «manne africaine » alimente non seulement les deux Narbonnaises mais également des villes très éloignées du littoral méditerranéen, comme Lyon (voir T. Silvino, notice 3, infra, p. 153-155) et Bordeaux (81\%, voir Amiel, Berthault, 1996, tabl. II). Les importations en provenance d'Espagne connaissent une certaine recrudescence, surtout dans les villes : huile de Bétique (amphores Dressel 20 tardives et Dressel 23 présentes à Arles, voir J. Piton, notice 4, infra, p. 155) et salaisons de poissons de même provenance ou de Lusitanie (amphores Almagro 50, 51a-b et 51c sur l'épave de Port-Vendres I) ; elles peuvent même dominer les importations africaines dans les villes proches de l'Espagne (61\% contre $38 \%$ à Toulouse, voir Amiel, Berthault, 1996, tabl. I ; voir F. Berthault, notice 2, infra, p. 152-153). Comme on l'a vu, le vin italien reste présent avec les amphores siciliennes Ostia I, 456 (pour Arles, voir J. Piton, notice 4, infra, p. 155). Le vin oriental est représenté, comme aux siècles précédents, par quelques importations de Crète et d'Asie Mineure (conteneurs mono-ansés de la région d'Éphèse à Lyon).

\section{LA CROISSANCE DES AMPHORES ORIENTALES AU $\mathbf{V}^{\mathbf{e}} \mathrm{S}$.}

Dès le début $d u \mathrm{~V}^{\mathrm{e}} \mathrm{s}$., les amphores africaines perdent leur relative hégémonie. Ce recul profite aux conteneurs hispaniques (Dressel 23, Almagro 51a-b) dans la partie ouest de la Narbonnaise (voir F. Berthault, notice 2, infra, p. 152-153), comme à Toulouse (70\% d'hispaniques contre $16 \%$ d'africaines, voir Amiel, Berthault, 1996, tabl. I) ou à Narbonne (basilique du Clos de la Lombarde : $44 \%$ contre 32 \%, voir Raynaud, 1991b, p. 244 ; Hôtel-Dieu : $68 \%$ contre $25 \%$, voir Ginouvez dir., 1996-1997, fig. 47), mais il est dû surtout à une augmentation spectaculaire des importations de Méditerranée orientale. Durant la majeure partie du V ${ }^{\mathrm{e}}$ s., de part et d'autre du Rhône (Beaucaire : Raynaud, 1987 ; Arles : Congès, Leguilloux, 1991 ; Marseille : Bonifay, Pieri, 1995, p. 116), les amphores orientales atteignent en moyenne $45 \%$ du total des individus (contre $30 \%$ à l'Afrique). Cette tendance est également sensible à Narbonne (Alessandri et al., 1998, p. 121: 50 \% d'amphores hispaniques mais déjà $30 \%$ d'amphores orientales), à Toulouse (Amiel, Berthault, 1996, tabl. I : 70 \% d'hispani- ques pour $16 \%$ d'africaines et $12 \%$ d'orientales) et même à Bordeaux (id., ibid., tabl. II : $9 \%$ ). Il s'agit principalement d'amphores vinaires LRA 1 de Cilicie, LRA 3 d'Asie Mineure et $L R A 4$ de Gaza. On reste mal renseigné sur le mode d'acheminement de ces amphores en Gaule: une seule épave, celle de la Redoute Béar à Port-Vendres (Castellvi et al., 2003), semble comporter un chargement principal d'amphores orientales (type $L R A 3$, associé à des exemplaires moins nombreux de type $L R A 1$ et $L R A 4$, et à quelques amphores africaines et italiques).

Certes, des calculs intégrant la capacité des amphores permettent de voir que les quantités de produits orientaux et africains s'équilibrent (Bonifay, Villedieu, 1989, p. 39), ces derniers arrivant dans des conteneurs de très grandes dimensions. Mais la nature des importations africaines n'est peut-être pas comparable ; elle est en tout cas plus diversifiée, garum (type Keay 35B ?), huile (Keay 35A), olives en conserve (Keay 25/spatheia), comme le montre la composition du chargement de l'épave Dramont $E$ au deuxième quart du V $\mathrm{V}^{\mathrm{e}}$ s. (Santamaria, 1995).

En revanche, le vin italien reste bien représenté, notamment à Marseille, avec le type Keay 52 originaire de Calabre (Bonifay, Pieri, 1995, p. 116).

\section{REPRISE AFRICAINE ET DIVERSIFICATION DES FLUX ORIENTAUX AU VI ${ }^{\mathrm{e}} \mathrm{S}$.}

Les données chiffrées pour le VI ${ }^{\mathrm{e}} \mathrm{s}$. concernent presque exclusivement Marseille. On note dans cette ville une nette reprise des importations africaines, avec l'arrivée de nouvelles générations de conteneurs (types Keay 55 et 62), dont la proportion s'équilibre avec celle des amphores orientales (Pieri, 2005 : $30 \%$ et $30 \%$ dès le début du $\mathrm{VI}^{\mathrm{e}} \mathrm{s}$. ) ; cette tendance persiste (variantes tardives du type Keay 62 et type 61C) jusque dans le premier quart du VII ${ }^{\mathrm{e}} \mathrm{s}$. (ibid. : $40 \%$ pour chacune des deux productions). Ces amphores, bien attestées en milieu urbain, portuaire (Toulon : Bérato et al., 1986 ; Bordeaux : Berthault, 1999, fig. 17, $\mathrm{n}^{\text {os }} 1-2$ ) ou non (Nîmes: Raynaud dir., 1990, fig. 128, no 97), sont également présentes sur des sites ruraux (Eyguières: Pelletier et al., 2000, fig. 41, no 4 ; Camargue : Tréglia, 2004, fig. 1, $\mathrm{n}^{\mathrm{O}} 1$ ) parfois éloignés des côtes (Séviac à Montréal : Amiel, Berthault, 1996, p. 258), et même dans les habitats de hauteur (Saint-Blaise : Villedieu, 1994, fig. 79, $\mathrm{n}^{\text {os }} 12-16$; Constantine à Lançon-de-Provence: Verdin, 2001, fig. 8, $\mathrm{n}^{\circ} 20$ et fig. 9, $\mathrm{n}^{\mathrm{o}} 19$; Roc de Pampelune, voir la contribution de L. Schneider, supra, p. 11-56; Roc d'Enclar : Llovera Massana et al., 1997, fig. 133, $\mathrm{n}^{\text {os }} 1$ et 3 , et fig. 134, $\mathrm{n}^{\circ} 1$ ). 
Le contenu de ces amphores reste controversé mais il s'agirait plus vraisemblablement de garum ou de vin, que d'huile (Bonifay, 2004, tabl. IV).

Du point de vue des amphores orientales, on constate une diversification des sources d'approvisionnement, la Grèce continentale (type $L R A 2$ ) et la région syro-palestinienne (bag shaped amphoras, LRA 5/6) venant s'ajouter aux précédentes. Le vin semble toujours être la denrée transportée dans ces amphores.

L'épave de la Paludà Hyères (Long, Volpe, 1998), datée du deuxième quart $\mathrm{du} \mathrm{VI}^{\mathrm{e}} \mathrm{s}$., est peut-être révélatrice du mode de commercialisation de ces conteneurs: une cargaison principale d'amphores africaines Keay 55 et 62, associée à une cargaison secondaire d'amphores orientales $L R A 1,2$, 4 et 5 . En revanche, la situation particulière de Bordeaux où, comme en Grande-Bretagne, les amphores orientales surpassent les amphores africaines (Amiel, Berthault, 1996, tabl. II : $24 \%$ contre $4 \%$ ), révèle peut-être l'existence de contacts directs avec le monde byzantin, par le détroit de Gibraltar (Berthault, 1999, p. 285).

\section{LA CONTINUITÉ DES ARRIVAGES D'AMPHORES JUSQU'À LA FIN DU VII ${ }^{\mathrm{E}}$ S. OU LE DÉBUT DU VIII ${ }^{\mathrm{E}} \mathrm{S}$.}

L'avancée peut-être la plus spectaculaire concerne le $\mathrm{VII}^{\mathrm{e}} \mathrm{s}$. La documentation s'est considérablement accrue à Marseille avec les fouilles de la place Jules-Verne (Bonifay, Pieri, 1995, annexes II et III ; voir S. Bien, notice 5, infra, p. 156-157), de la place Villeneuve-Bargemon (Bien, 2003) et de l'Alcazar (Bien, 2005a). Les tendances qui avaient été enregistrées en 1986 (Bonifay, 1986) se trouvent confirmées, notamment celles concernant la suprématie des arrivages africains (types Keay 8A, 50 et 61A/D ; spatheia miniatures ; amphores globulaires) à la fin du VII ${ }^{\mathrm{e}} \mathrm{s}$. (75\% d'amphores africaines sur le chantier de la place Jules-Verne, voir Pieri, 2005 ; même pourcentage sur le chantier de l'Alcazar, voir Bien, 2005a, fig. 1, état 2). En revanche, les décennies centrales du $\mathrm{VII}^{\mathrm{e}} \mathrm{s}$. dessinent peut-être une situation plus nuancée avec encore plus de $30 \%$ d'amphores orientales (place Villeneuve-Bargemon: Bien, 2003, tabl. 6) ; ces dernières proviennent majoritairement de régions passées dès 640 sous la domination arabe: amphores de Cilicie (type $L R A 1$ ), de Syrie-Palestine ( $L R A 5 / 6)$, de Gaza ( $L R A 4)$ et d'Égypte ( $L R A 7$ et imitations de $L R A 5)$. Il en est de même, à partir de 675 , des productions africaines du Sahel tunisien, et, à partir des années 690, pour toutes les autres productions. Il n'est pas impossible, en effet, que ces importations se poursuivent durant les premières décennies du VIII ${ }^{\mathrm{e}}$ s., comme tendraient à le prouver, en milieu rural, les contextes de San Peyre à Suzon (types Keay 8A et 61A/D, voir Pellecuer, 1999) et de Dassargues (amphores globulaires : CATHMA, 1993, p. 177).

Ces découvertes font écho à celles de Rome (Crypta Balbi : Saguì dir., 1998), de Sant'Antonino di Perti (Mannoni, Murialdo dir., 2001) et de Tarragone (Macias i Sole, Remolà i Vallverdu, 2000) et à l'épave Saint-Gervais 2, actuellement l'une des plus tardives de Méditerranée (Jézégou, 1998).

Ces importations du VII ${ }^{\mathrm{e}} \mathrm{s}$. apparaissent également, bien que de manière plus sporadique, à Bordeaux (type Keay 61, Berthault, 1999, fig. 17, no 4 et notice 2, infra, p. 152-153) et à Lyon (voir T. Silvino, notice 3, infra, p. 153-155).

Le commerce des amphores, même s'il n'englobe pas tout le commerce de l'Antiquité tardive, reste donc une réalité en Gaule du Sud jusqu'à la fin du VII ${ }^{\mathrm{e}} \mathrm{s}$. ou au début du VIII ${ }^{\mathrm{e}} \mathrm{s}$, principalement sur le littoral méditerranéen, à la fois en milieu urbain et en milieu rural, mais également dans les grandes villes plus septentrionales (Lyon et Bordeaux). Comme aux époques antérieures, les échanges les plus visibles concernent le vin, avec peutêtre des arrivages importants d'Afrique au $\mathrm{IV}^{\mathrm{e}} \mathrm{s}$. et de Méditerranée orientale au $\mathrm{V}^{\mathrm{e}} \mathrm{s}$., associés à un flux italien minoritaire mais constant. Le vin oriental et probablement aussi celui provenant d'Afrique sont attestés jusqu'au début du VIII ${ }^{\mathrm{e}}$ s. Les salsamenta sont encore l'affaire de la péninsule Ibérique $\mathrm{au} \mathrm{IV}^{\mathrm{e}} \mathrm{s}$. et au début du $\mathrm{V}^{\mathrm{e}} \mathrm{s}$., mais ils deviennent probablement exclusivement africains à partir du milieu de ce siècle. Les arrivages d'huile, plus difficiles à mesurer, semblent suivre la même évolution.

M. B.

\section{LA PRÉSENCE DES VAISSELLES IMPORTÉES}

S'il est un caractère qui distingue la Gaule méridionale des régions plus septentrionales durant l'Antiquité tardive, c'est bien la présence régulière, jusqu'à une date tardive, des vaisselles importées. Ces objets jouent un rôle déterminant du point de vue de la chronologie, celui d'un " pivot» permettant de dater les productions locales, rôle plus traditionnellement dévolu aux mobiliers métalliques en Gaule septentrionale. Mais on doit tout d'abord s'interroger sur la signification de la présence de ces céramiques du point de vue de l'histoire des échanges avant d'envisager son impact sur la consommation, du $\mathrm{IV}^{\mathrm{e}}$ au VII ${ }^{\mathrm{e}} \mathrm{s}$. (voir S. Bien, notice 5, infra, p. 156-157). 


\section{QUELLE SIGNIFICATION DONNER À LA PRÉSENCE DES VAISSELLES IMPORTÉES ?}

Selon un schéma commun à l'ensemble du bassin occidental de la Méditerranée, la forte présence des vaisselles importées en Gaule du Sud est une situation qui prévaut dès le II $^{\mathrm{e}} \mathrm{s}$. Elle est principalement due à l'afflux de vaisselles africaines, culinaires dès l'époque flavienne et sigillées A surtout à partir des Antonins, auxquelles s'ajoutent, au III ${ }^{\mathrm{e}}$ s., les productions de Tunisie centrale (sigillée africaine C). Dès cette époque, les céramiques africaines atteignent près de $15 \%$ du total de la vaisselle dans les campagnes du Languedoc oriental (Raynaud, 2007) et dominent toutes les autres catégories dans une ville comme Marseille (Lang, 1998a, tabl. VIII-IX).

Contrairement à une hypothèse solidement établie, il est douteux que les sigillées et les amphores africaines aient été commercialisées ensemble. Les courbes inversées des importations de sigillées et d'amphores africaines en Languedoc oriental (Raynaud dir., 1990, fig. 144 et 145) font écho à la situation de la Méditerranée orientale où l'on a remarqué de longue date « la dissociation entre les vaisselles fines (et les lampes) et les amphores africaines » (Sodini, 2000, p. 193). De fait, on ne connaît pas, pour les $\mathrm{II}^{\mathrm{e}}-\mathrm{IV}^{\mathrm{e}} \mathrm{s}$., d'épave avec une cargaison composée à la fois d'amphores et de vaisselle africaine. Dès lors, on peut se demander si l'explication alléguée en Orient - la sigillée africaine aurait voyagé avec les convois de blé (Panella, 1993) - ne pourrait s'appliquer également à la Méditerranée occidentale (Bonifay, 2003) ? Dans cette hypothèse, les grands ports dotés d'entrepôts auraient joué un rôle important dans la diffusion des sigillées africaines.

La même remarque peut être faite à propos des vaisselles de Méditerranée orientale (sigillées phocéennes et surtout céramiques culinaires de mer Égée, du Levant et de Constantinople) : elles sont peu nombreuses au $\mathrm{V}^{\mathrm{e}} \mathrm{s}$., au moment de l'explosion des importations d'amphores orientales, tandis que leur présence augmente notablement aux $\mathrm{VI}^{\mathrm{e}}-\mathrm{VII}^{\mathrm{e}} \mathrm{s}$. quand celle des amphores diminue. Au VII ${ }^{\mathrm{e}} \mathrm{s}$, sur le chantier de l'Alcazar à Marseille, les céramiques communes orientales dominent l'ensemble des céramiques communes importées (Bien, 2005a). Dans ce cas comme dans celui des productions africaines, les importations de vaisselle trahissent peut-être des arrivages de denrées dont la vente était certainement plus lucrative mais qui n'ont pas laissé de trace archéologique : épices, étoffes, etc. (voir supra, p. 97).
Enfin, on a noté en introduction la présence d'importations de vaisselles de Gaule du Nord, anecdotique jusqu'à la fin $\mathrm{du} \mathrm{VI}^{\mathrm{e}} \mathrm{s}$. (métallescente, sigillée d'Argonne) mais plus conséquente à partir de cette date (céramique commune à pâte bistre du Val de Saône). Ces dernières productions qui apparaissent sur des sites également bien irrigués par les importations méditerranéennes font écho aux découvertes toujours plus nombreuses d'amphores orientales, de sigillées et de lampes africaines en Gaule du Nord et dont il reste à dresser une cartographie.

M. B.

\section{LES SIGILLÉES MÉDITERRANÉENNES}

Les sigillées africaines (fig. 53) fournissent l'essentiel des contingents aux côtés d'arrivages plus modestes de sigillées phocéennes tardives et de quelques importations sporadiques de productions chypriotes et égyptiennes (fig. 54).

\section{LES SIGILLÉES AFRICAINES}

Les recherches sur les ateliers de sigillées africaines ont considérablement progressé depuis le milieu des années 1980. En effet, jusqu'à cette date, on ne connaissait guère sur le terrain que les ateliers de Sidi Aïch et de Henchir es Srira (Stern, 1968) dont la production, essentiellement continentale (diffusée en Algérie), n'a été qu'exceptionnellement exportée outre-mer (un fragment d'assiette Stern VII de Sidi Aïch a été retrouvé à Marseille). Ce sont tout d'abord les prospections menées en Byzacène qui ont permis de mettre en évidence l'un des plus importants ateliers de sigillée africaine C, Sidi Marzouk Tounsi (Peacock et al., 1990), dont les analyses physico-chimiques montrent actuellement qu'il est probablement à l'origine de la plupart des produits exportés en Méditerranée (Mackensen, Schneider, 2002) ; cet atelier, d'où provient également une partie des lampes en sigillée de type Atlante X (groupes C2-4: Bonifay, 2004, p. 80 et fig. 41), a été actif du III $^{\mathrm{e}} \mathrm{s}$. jusqu'aux décennies centrales du $\mathrm{VI}^{\mathrm{e}}$ s. (Mackensen, 2004, p. 133). Le fait le plus marquant a consisté ensuite dans la publication (Mackensen, 1993) de l'atelier d'El Mahrine, dans la basse vallée de la Mejerda, qui est sans doute le grand centre de production de la sigillée africaine D1 (pour la définition de cette catégorie, voir Carandini et al., 1981). Cet atelier est créé vers les années 320-330 et produit jusqu'à la fin du VII ${ }^{\mathrm{e}} \mathrm{s}$. (Mackensen 2004, p. 150), mais il est surtout représenté sur les marchés méditerranéens $\mathrm{au} \mathrm{IV}^{\mathrm{e}} \mathrm{s}$. et dans la première moitié $\mathrm{du} \mathrm{V}^{\mathrm{e}} \mathrm{s}$. (formes Hayes 59, 61A, 67, 76). De même, les recherches 


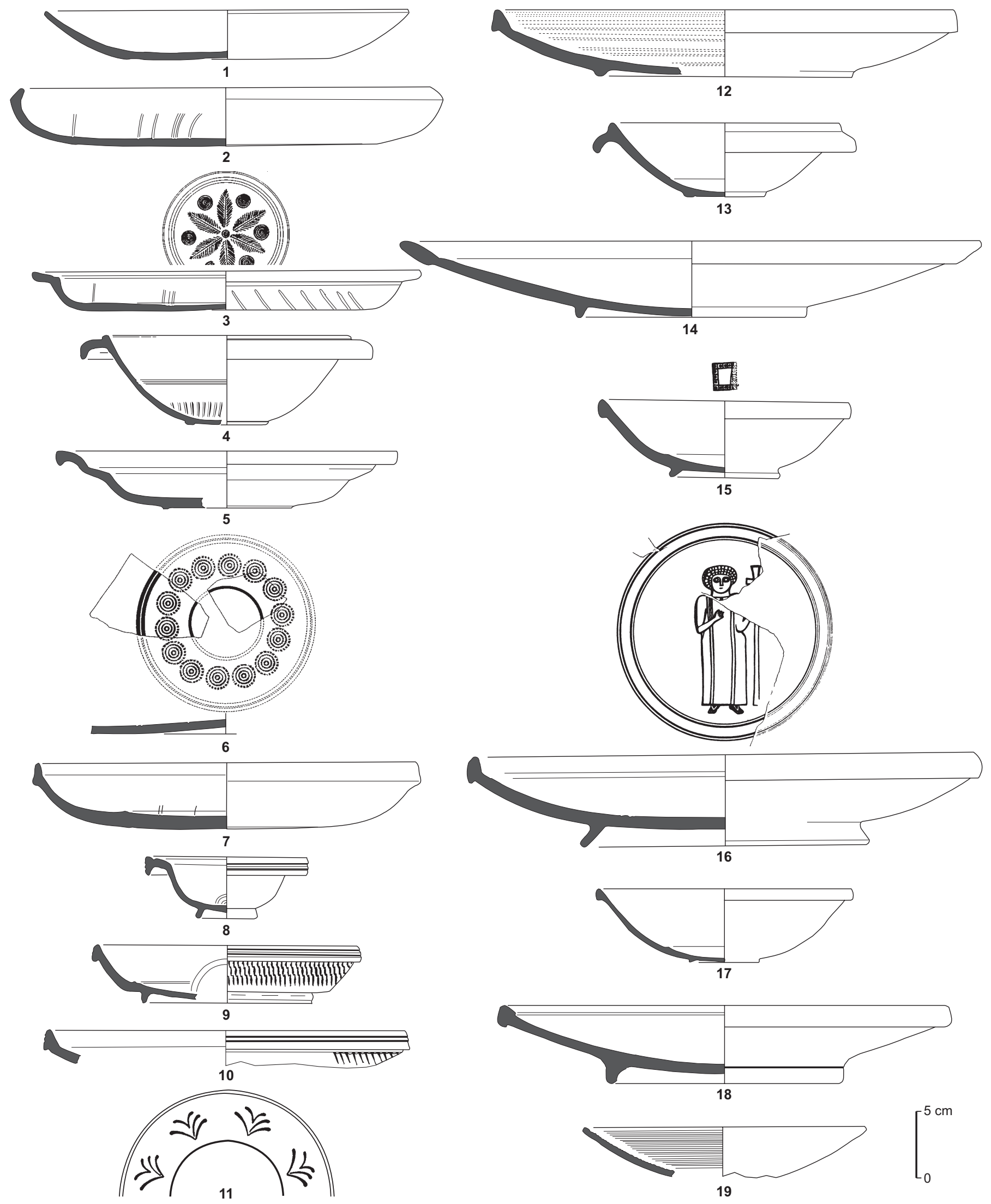


tuniso-françaises menées à Oudhna à partir de 1995 ont permis de reprendre l'étude de cet important atelier de sigillée D2, signalé dès 1896, dont les productions (principalement les formes Hayes 91C et 95-99) sont bien diffusées en Méditerranée aux VI ${ }^{\mathrm{e}}-\mathrm{VII}{ }^{\mathrm{e}} \mathrm{s}$. (Barraud et al., 1998). D'autres ateliers ont été entrevus, celui de Sidi Khalifa, dans le golfe d'Hammamet, dont la production est d'une qualité intermédiaire entre les catégories $\mathrm{C}$ et $\mathrm{D}$ (formes Hayes 61B3, 86, $87 \mathrm{~A}$ et surtout 88, voir Bonifay, 2004, p. 49 et p. 55-56), qui fut actif surtout dans la seconde moitié $d u \mathrm{~V}^{\mathrm{e}} \mathrm{s}$. et la première moitié du $\mathrm{VI}^{\mathrm{e}}$ s., et un atelier mineur de la région de Nabeul Sidi Zahruni, déjà mentionné pour sa production d'amphores (Ghalia et al., 2005), qui a exporté au $\mathrm{V}^{\mathrm{e}} \mathrm{s}$. des variantes locales de formes standard (formes Hayes 50B late variant et Fulford 27). M. Mackensen a également mis en évidence, sur des bases stylistiques, un atelier qui n'a pas encore été reconnu sur le terrain mais qui se situe peut-être dans la région de El Fahs, Zaghouan et Oudhna (Mackensen, 1998), à l'origine de la majeure partie des plats Hayes 103-104 décorés selon le style $\mathrm{E}$ (ii). On reste mal renseigné sur la production de la catégorie E (Carandini et al., 1981), caracté-

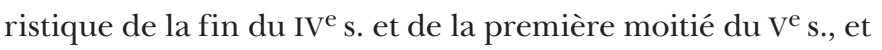
faiblement diffusée en Méditerranée (forme Hayes 68), dont l'un des ateliers paraît cependant avoir été reconnu à Djilma (Mackensen, 1993) et sur celle des formes les plus tardives de la production D (Hayes 105 et 109). Enfin, on ignore tout de la localisation des ateliers de sigillée africaine A mais cette catégorie, en voie de disparition dans la seconde moitié du $\mathrm{III}^{\mathrm{e}}$ s., reste hors des limites chronologiques que nous nous sommes fixées. Est-il possible de reconnaître ces différentes productions au sein du matériel recueilli en Gaule du Sud?

\section{L'afflux des sigillées africaines D au $\operatorname{IV}^{\mathrm{e}} \mathrm{s}$. et au début du $\mathrm{V}^{\mathrm{e}} \mathrm{s}$.}

La Gaule du Sud n'échappe pas au développement considérable des importations de sigillée africaine constaté partout en Méditerranée au IV s. (Sodini, 2000, p. 188).
Prenant la place des sigillées du centre de la Tunisie, les productions des ateliers de la Mejerda (catégorie D1) envahissent, à partir des décennies centrales du $\mathrm{IV}^{\mathrm{e}} \mathrm{s}$., la plupart des sites urbains et ruraux. À Arles, malgré les arrivages de sigillées luisantes, cette vaisselle domine tous les contextes de la seconde moitié du $\mathrm{IV}^{\mathrm{e}} \mathrm{s}$. (formes Hayes 58, 59, 61A) (voir J. Piton, notice 4, infra, p. 155) ; en Languedoc oriental, la sigillée africaine atteint $32 \%$ des vaisselles fines au début $d u \mathrm{~V}^{\mathrm{e}} \mathrm{s}$. (Raynaud, 2007). Dans le premier quart du $\mathrm{V}^{\mathrm{e}} \mathrm{s}$., les sigillées africaines dominent encore à Arles (Congès, Leguilloux, 1991) et font part égale avec les Dérivées-de-Sigillées Paléochrétiennes (DS.P.) à Narbonne (Ginouvez dir., 1996-1997, fig. 48) et à Marseille (chantier de la Bourse, contexte 21 : Cœur-Mezzoud, 1998, tabl. XXVIII et XXXIII), alors que les amphores africaines ont déjà cédé du terrain face aux importations orientales et hispaniques.

\section{Le déclin des sigillées africaines au milieu et dans la seconde moitié du $\mathrm{V}^{\mathrm{e}} \mathrm{s}$.}

La proportion des sigillées africaines décline au milieu $\mathrm{du} \mathrm{V}^{\mathrm{e}} \mathrm{s}$. À Marseille, elles ne représentent plus que 6 à $16 \%$ du total de la vaisselle (De Mitri, 2001, tabl. 1). Durant la seconde moitié $\mathrm{du} \mathrm{V}^{\mathrm{e}} \mathrm{s}$, les sigillées africaines vont conserver ce faible taux : $11 \%$ dans le comblement du puits de la rue du Bon-Jésus à Marseille (Reynaud et al., 1998). On observe également un changement dans les sources d'approvisionnement, les productions du nord de la Tunisie étant remplacées sur les marchés gaulois par celles de Byzacène centrale (production C5 des ateliers de Sidi Marzouk Tounsi : formes Hayes 82-85) et du nord du golfe d'Hammamet (notamment atelier de Sidi Khalifa: formes Hayes $61 \mathrm{~B}$ et $87 \mathrm{~A}$ ). En outre, ces produits ne sont pas régulièrement répartis sur le territoire : bien attestés dans les villes et quelques grandes exploitations agricoles (La

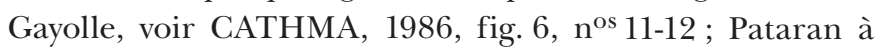
Aigues-Vives, ibid., nº 6 ; Gardanne, voir Pelletier et al., 1991,

Fig. 53 - Principales formes de sigillées africaines attestées en Gaule du Sud: 1, forme Hayes 50B, Lunel-Viel (d'après Raynaud, 1984b); 2, forme Hayes 61A, Lansargues (d'après Girard, Raynaud, 1982) ; 3, forme Hayes 59A, Narbonne (d'après Sabrié, Sabrié, 1991) ; 4, forme Hayes 91A, Marseille, la Bourse (d'après Bonifay, 2004) ; 5, forme Hayes 67, Aix-en-Provence (d'après Guild et al., 1980) ; 6, décor style A(iii), Saint-Blaise (d'après Rivet, 2001) ; 7, forme Hayes 61B, épave Dramont E (d'après Santamaria, 1995) ; 8, forme Hayes 74, Marseille (d'après Bonifay, 1998) ; 9, forme Hayes 84, Marseille (ibid.) ; 10, Marseille, la Bourse (d'après Bonifay, Pelletier, 1983) ; 11, décor lustré, dépotoir de l'anse Gerbal à Port-Vendres (d'après Martin, 1977) ; 12, forme Hayes 87A, Saint-Blaise (d'après Cavaillès-Llopis, Vallauri, 1994) ; 13, forme Hayes 91B, Velaux, Sainte-Propice (d'après Bonifay, Rivet in Boixadera et al., 1987) ; 14, forme Hayes 88, Saint-Blaise (d'après CavaillèsLlopis, Vallauri, 1994) ; 15, forme Hayes 99B, Marseille (d'après Gourvest, 1998) ; 16, forme Hayes 104A, Velaux, Sainte-Propice (d'après Bonifay, Rivet in Boixadera et al., 1987) ; 17, forme Hayes 80B/99, Marseille, la Bourse (d'après Bonifay, 2004) ; 18, forme Hayes 105, Marseille, la Bourse (ibid.) ; 19, forme Hayes 109, Marseille, pavillon Bargemon (d'après Bien, 2003) (DAO : M. Bonifay, CNRS). 
fig. 8, $\mathrm{n}^{\text {os }} 6$-12), voire sur des sites de hauteur (Saint-Blaise, voir Cavaillès-Llopis, Vallauri, 1994, fig. 48 et 49), ils disparaissent entièrement de certaines régions (aucun témoin à Lunel-Viel). Le déclin des sigillées africaines profite aux DS.P. qui, à Marseille comme en Languedoc, constituent un phénomène du milieu et de la seconde moitié du $\mathrm{V}^{\mathrm{e}} \mathrm{s}$., mais on ignore si le développement de ces dernières est la cause de l'affaiblissement des premières ou bien si les DS.P. ont comblé le vide créé par la baisse des importations de vaisselle africaine. En somme, on ne sait comment trancher entre une explication conjoncturelle privilégiant les conditions locales du marché et une explication plus structurelle prenant en compte les conditions générales du commerce méditerranéen. On doit en effet rappeler que la baisse des importations de sigillées africaines est générale en Méditerranée dans la seconde moitié du V $\mathrm{V}^{\mathrm{e}} \mathrm{s}$.

\section{Recrudescence, limitée au littoral, de la présence des sigillées africaines au $\mathrm{VI}^{\mathrm{e}} \mathrm{s}$. et au début du VII ${ }^{\mathrm{e}} \mathrm{s}$.}

On assiste $\mathrm{au} \mathrm{VI}^{\mathrm{e}} \mathrm{s}$. à une double tendance. D'une part, la diffusion de la sigillée africaine en Gaule tend à se restreindre aux sites les plus importants (grandes villes) ou les plus proches du littoral. D'autre part, sur ces sites sa proportion est en hausse. Il est remarquable que cette céramique soit particulièrement bien attestée sur les sites de hauteur (plus de la moitié du total des vaisselles fines de Sainte-Propice à Velaux, voir Boixadera et al., 1987, fig. 7). L'origine des importations change à nouveau : les produits de Byzacène disparaissent pour laisser place à ceux de l'atelier d'Oudhna (forme Hayes 99, présente jusqu'à Lyon et Bordeaux) et de cet atelier non encore localisé qui a produit les plats Hayes 104 (cette forme est bien attestée sur le site de hauteur de Pampelune). À Marseille, les taux les plus forts sont atteints à la fin du VI ${ }^{\mathrm{e}} \mathrm{s}$. et au début du VII ${ }^{\mathrm{e}} \mathrm{s}$., avec les formes Hayes 99B et C, 90B, 105 et 109A (Bonifay, Pelletier, 1983) ; le même faciès mais avec un nombre réduit de tessons se retrouve jusqu'à Bordeaux (fouilles de la place Camille-Jullian, inédit).

\section{Les dernières importations de sigillées africaines dans la seconde moitié du VII ${ }^{\mathrm{e}} \mathrm{s}$.}

Les contextes de la seconde moitié du VII ${ }^{\mathrm{e}} \mathrm{s}$. comportant des sigillées africaines se rencontrent principalement à Marseille (place Jules-Verne : Bonifay, 1998, fig. 3, $\mathrm{n}^{\circ} 6$; place Villeneuve-Bargemon: Bien, 2003; Alcazar: Bien, 2005a). Les principaux marqueurs chronologiques sont les
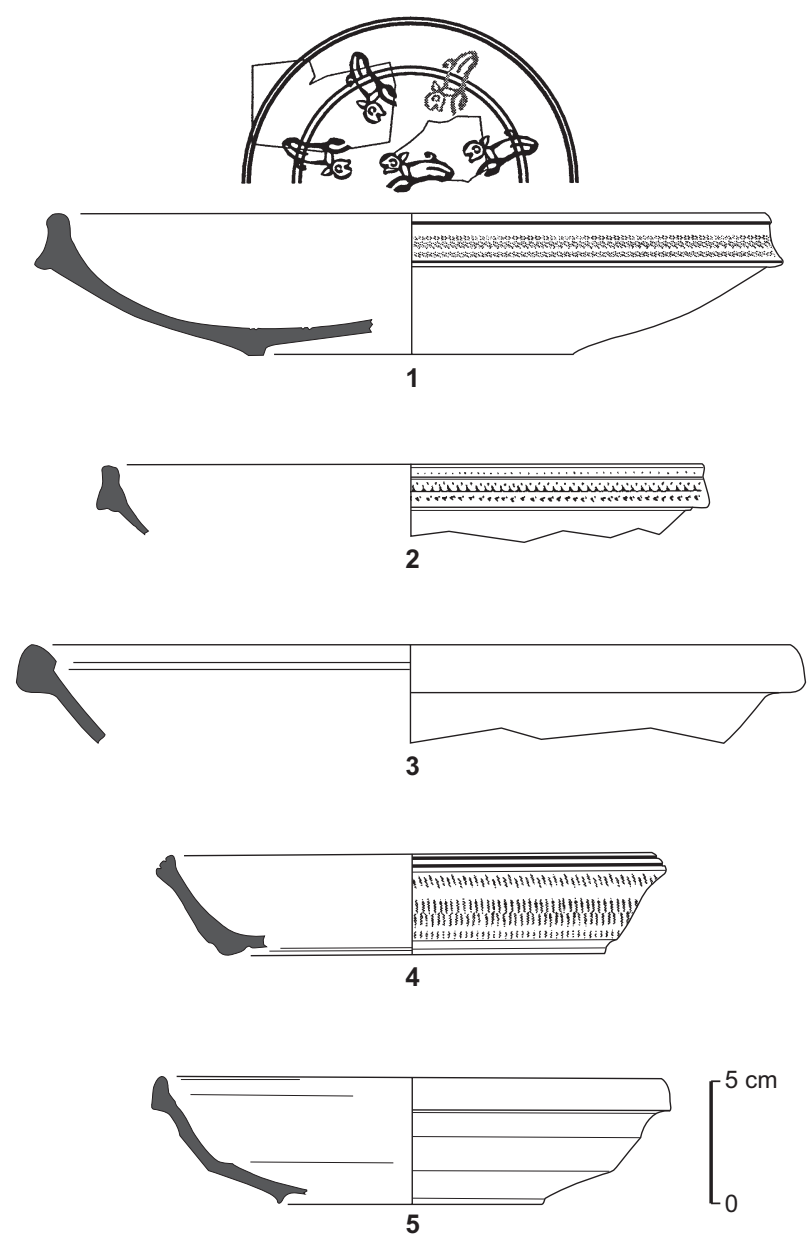

Fig. 54 - Sigillée phocéenne : 1, forme Hayes 3C, Saint-Blaise (d'après Rivet, 2001) ; 2, forme Hayes 3D, Bordeaux (d'après Bonifay, à paraître) ; 3, forme Hayes 10A, Marseille, la Bourse (d'après Bonifay, Pelletier, 1983, fig. 30, no 195). Sigillée chypriote tardive : 4, forme Hayes 2, Saint-Blaise (d'après Rivet, 2001). Sigillée égyptienne A (Assouan) : 5, forme Gempeler T324, Marseille, pavillon Bargemon (d'après Bien, 2003) (DAO : M. Bonifay, CNRS).

plats Hayes 109B (Bonifay, 1998) et les variantes les plus tardives de bols Hayes 99 (variante 80B/99). L'épave SaintGervais 2 semble appartenir au même horizon chronologique (Jézégou, 1998, fig. 308, no 11 : Hayes 109B). Parmi les productions africaines les plus tardivement importées, on trouve également des lampes de type Atlante $\mathrm{X}$ au décor surmoulé (Saint-Gervais 2: Jézégou, 1998, fig. 308, nº 12 ; Mont Bouquet: $C A G, 30 / 2$, fig. 241).

\section{LES SIGILLÉES PHOCÉENNES TARDIVES}

Ces céramiques (Late Roman C ware de Waagé, 1948) originaires de la côte occidentale de l'Asie Mineure, prin- 
cipalement autour de Phocée (Mayet, Picon, 1986), sont diffusées, bien que toujours en faible quantité, sur un bon nombre de sites du littoral (CATHMA, 1991, p. 28 et fig. 2) : Antibes, Fréjus (Béraud et al., 1991, fig. 49A, no 1), La Gayole, Olbia à Hyères, Toulon, Saint-Estève-de-Ménerbes, Marseille (Bonifay et al. dir., 1998, p. 365), Gardanne (Pelletier et al., 1991, fig. 7), Aix-en-Provence, Constantine à Lançon-de-Provence, Saint-Julien-lès-Martigues, SaintBlaise (Cavaillès-Llopis, Vallauri, 1994, fig. 66 ; Rivet, 2001, fig. 3), Arles, Saint-Laurent-d'Aigouze, Port-Vendres. Des témoins ont été retrouvés jusqu'à Bordeaux (fouilles de la place Camille-Jullian, inédit).

À Marseille, ces productions apparaissent dans la seconde moitié $d u \mathrm{~V}^{\mathrm{e}} \mathrm{s}$. avec les formes Hayes 1D et 3C, mais elles sont surtout fréquentes au $\mathrm{VI}^{\mathrm{e}} \mathrm{s}$., avec les formes $3 \mathrm{D}$, E et $\mathrm{F}$ (Bonifay et al. dir., 1998, p. 365). Une forme 10B issue d'un contexte du VII ${ }^{\mathrm{e}}$ s. (place Jules-Verne : Bonifay, Pieri, 1995, annexe III) est l'attestation la plus tardive de l'importation de cette céramique en Gaule du Sud.

\section{AUTRES SIGILLÉES ORIENTALES}

Les sigillées chypriotes tardives (Late Roman D ware de Waagé) sont relativement rares en Gaule du Sud. Trois sites ont livré ce type de céramique : Marseille (Bonifay et al. dir., 1998, p. 365 : formes Hayes 2 et 5), Saint-Blaise (Rivet, 2001, fig. 5 : formes Hayes 1 ?, 2 et 9), Port-Vendres (dépotoir de l'anse Gerbal : Bonifay, et al. dir., 1998 : formes 2, 5, 7 et 11). Ces importations sporadiques couvrent la seconde moitié $\mathrm{du} \mathrm{V}^{\mathrm{e}}$ s. et tout le $\mathrm{VI}^{\mathrm{e}} \mathrm{s}$.

Enfin, les niveaux du $\mathrm{VII}^{\mathrm{e}} \mathrm{s}$. de Marseille ont livré récemment quelques témoins de sigillée égyptienne $\mathrm{A}$ d'Assouan (Bien, 2003, fig. 4, no 12) qui constituent les exportations les plus lointaines, connues à ce jour, de ce type de céramique.

M. B.

\section{LES VAISSELLES COMMUNES D'IMPORTATION MÉDITERRANÉENNE}

Depuis les premières enquêtes (CATHMA, 1991 ; Raynaud, 1993a) qui, vers la fin des années 1980, révélèrent leur présence en Gaule du Sud, le répertoire typologique des céramiques communes importées n'a cessé de s'enrichir, à la faveur notamment des ensembles de mobilier découverts durant les fouilles préventives réalisées ces dernières années à Marseille (place Jules-Verne, place Villeneuve-Bargemon, Alcazar, tunnel de la Major). La collaboration accrue entre archéomètres et archéologues autour des problématiques relatives à la détermination de leur origine permet de disposer aujourd'hui d'une classification régionale des productions : Égypte, Levant, mer Égée, mer de Marmara, Afrique du Nord, Italie du Sud, Latium, Ligurie, Espagne du Sud (voir J.-C. Tréglia, notice 6, infra, p. 157-159) (fig. 83). Si l'attrait gaulois pour les sigillées de Carthage, de Phocée ou de Chypre se justifie par la qualité et l'originalité typologique de ces services de table, on peut s'interroger, en revanche, sur les raisons qui motivent l'importation de vaisselles communes adaptées à de très lointains usages culinaires (Arthur, 2007).

L'introduction d'ustensiles importés dans l'équipement des cuisines de Gaule méridionale s'inscrit de toute évidence dans une longue tradition et pourrait avoir été dictée par le besoin de corriger les fonctionnalités réduites des services utilitaires gaulois. Durant l'Antiquité tardive, aux petites ollae et cocottes à fond plat locales s'opposent en effet les vases de grand module, à paroi mince finement cannelée et à fond bombé importés depuis les rivages africains, égéens et levantins. De façon presque contradictoire, ce vaisselier d'outre-mer admet également de larges récipients culinaires massifs et modelés produits pour la plupart dans les archipels d'Italie du Sud (Pantelleria, îles éoliennes, Sardaigne). Contrairement aux productions gauloises, les caractères techniques de ces vases offraient probablement diverses opportunités de modes de cuisson (rissolé, à l'étouffé, friture) en tolérant peut-être, grâce à de meilleures propriétés réfractaires, une exposition directe au feu.

J.-C. T.

\section{L'AMPLE DIFFUSION DE LA CÉRAMIQUE BISTRE DU VAL DE SAÔNE}

Dénommée depuis quelques années céramique bistre en raison de sa coloration particulière, cette vaisselle à cuisson oxydante domine, du $\mathrm{V}^{\mathrm{e}}$ au VIII ${ }^{\mathrm{e}} \mathrm{s}$., sur une vaste région encadrant les vallées de la Saône, du Doubs et du Rhône, jusqu'au Jura suisse. En l'état des connaissances, l'une des principales aires de production est localisée dans la vallée de la Saône, à la latitude de Chalon-sur-Saône, en rive droite. Connue de longue date par des découvertes fortuites et des ramassages de surface, elle est décrite comme une céramique oxydée, dite "du groupe I de Sevrey ", dès les années 1970 (Renimel, 1974, p. 44-48, fig. 13-17). Si les indices de l'importance et de la pérennité des ateliers du secteur de Sevrey sont relevés dès cette époque (du haut Moyen Âge aux temps modernes), curieusement la question 
de la diffusion à moyenne et longue distance de cette céramique bistre est restée peu explorée jusqu'à ces toutes dernières années, la plupart des chercheurs des secteurs limitrophes regroupant les céramiques en pâte claire du haut Moyen Âge sous l'appellation générique de «produits du Val de Saône", contribuant à créer une confusion entre aire d'origine et aire de diffusion. Toutefois, durant les années 1990-2000, les connaissances ont progressé sur plusieurs fronts: à partir des sites de consommation, funéraires et domestiques, la typochronologie de cette production originale, et donc bien repérable, s'est considérablement perfectionnée en Rhône-Alpes (Horry, 2000 ; Faure-Boucharlat dir., 2001), en Bourgogne (Catteddu, 1992 ; Petident, Poil, 1998), en Franche-Comté (Collectif, 1995, p. 44-46 ; Billoin, 2003) et en Suisse (Haldimann, Steiner, 1996 ; Paratte-Rana et al., 1999) ; dans le même temps, les surveillances archéologiques reprenaient dans le secteur de Sevrey, offrant enfin l'occasion, en 2003, d'une fouille d'envergure sur un des sites de production (Delor Ahu, Simonin, 2006) ; parallèlement, une recherche de détermination et de diffusion de la céramique bistre était réalisée sous l'égide du laboratoire de céramologie de Lyon (CNRS-UMR 5138). Fondée sur une importante série d'analyses pétrographiques et chimiques, cette étude montre que la céramique bistre est issue non seulement des officines de Sevrey, mais aussi d'un ensemble assez disséminé d'ateliers contemporains, dits groupe de la forêt de la Ferté, qui laisse présager une dispersion encore plus vaste des lieux de production dans un Val de Saône élargi. Les produits de Sevrey, en particulier, et ceux du groupe des ateliers chalonnais, en général, atteignent tous les sites de consommation pris en compte par l'étude, dans l'Ain, la Côte-d'Or, le Rhône, l'Isère et le Jura français et suisse. Mais ils n'ont vraisemblablement pas le monopole de la vaisselle bistre : l'étude suggère que d'autres ateliers, encore à découvrir, ont alimenté les mêmes régions (Mani, 2005, p. 213-216). L'approche de la production et de la diffusion du désormais célèbre service bistre pourrait s'avérer plus complexe que ne le laissait supposer l'apparente suprématie des ateliers de Sevrey et du Chalonnais.

Les acquis récents ont également été essentiels en terme de chronologie, avec le concours de nombreuses datations numériques et la révision systématique des attributions traditionnellement admises. Son occurrence la plus précoce sur des sites de consommation se place dans la première partie $\mathrm{du} \mathrm{V}^{\mathrm{e}}$ s., à Lyon comme à Poncin, deux ensembles où elle apparaît en même temps que la sigillée grise (Ayala, 1998, p. 233 ; Faure-Boucharlat dir., 2001, p. 153-154). Sa mani- festation la plus récente est connue à la charnière des VIII ${ }^{\mathrm{e}}$ $\mathrm{IX}^{\mathrm{e}} \mathrm{s}$. toujours à Lyon, rue Pierre-Audry (Faure-Boucharlat dir., 2001, p. 383-388). Enfin, signalons que la phase de production de céramique bistre de l'officine fouillée récemment à Sevrey s'est étendue entre le deuxième tiers du $\mathrm{VI}^{\mathrm{e}} \mathrm{s}$. et le VIII ${ }^{\mathrm{e}}$ s. (Delor Ahu, Simonin, 2006, p. 84-85).

Une grande homogénéité technologique et morphologique caractérise donc cette production. Elle montre généralement une facture de qualité. De couleur beige orangé à brun-rouge, sa surface est fréquemment marquée de coups de feu. La pâte, siliceuse non calcaire, est en majorité granuleuse, comportant de nombreuses inclusions, feldspaths, quartz, oxydes de fer, micas. Sur les sites d'habitat, elle offre partout le même répertoire, composé essentiellement de quatre types : ollae (pots à cuire) piriformes ou globulaires à lèvre en bandeau ou en poulie, cruches à bec pincé, bols carénés et mortiers à collerette (fig. 55). La diversité des tailles, notamment parmi les formes ouvertes, atteste de la polyvalence de ces types principaux. Enfin, des décors imprimés à la molette ou de simples incisions, en particulier sur les ollae et les bols carénés, renforcent la spécificité de la céramique bistre. L'important échantillon provenant de l'officine de Sevrey a quelque peu enrichi ce répertoire, tant pour la table que pour la cuisine, avec des gobelets tronconiques et biconiques, des cruches à bec tubulaire, des marmites à deux anses, des couvercles et de curieux vases à anse de panier, autant de formes peu ou pas représentées dans les habitats (Delor Ahu, Simonin, 2006, pl. 1-7).

Si le service bistre est concurrencé, dans le Jura suisse, par d'autres catégories de céramiques (Parrate-Rana et al., 1999), notamment originaires d'Alsace, en revanche, en Franche-Comté, en Bresse et en Lyonnais, il représente à lui seul l'essentiel de la céramique oxydée aux VI ${ }^{\mathrm{e}}$ et VII ${ }^{\mathrm{e}} \mathrm{s}$. (Horry, 2000 ; Faure-Boucharlat dir., 2001 ; Billoin, 2003). Les données sont beaucoup moins claires au sud de Lyon. Certes, des céramiques bistres originaires du Chalonnais sont localisées depuis quelques années à Vienne, mais les choses restent à préciser pour cette ville qui semble marquer la limite méridionale d'une diffusion massive. Un fragment vient d'être identifié à Valence même (information orale de A. Horry), mais aucune trace n'en a été détectée dans la moyenne vallée du Rhône, de Valence à Pierrelatte, lors des fouilles du TGV Méditerranée (Horry, 2006). En fait, la surprise est venue de la région méditerranéenne où les céramiques bistres sont désormais attestées dans des contextes des VI ${ }^{\mathrm{e}}$ et VII ${ }^{\mathrm{e}}$ s. en Lunellois (Garnier et al., 1995, fig. 11, $\mathrm{n}^{\mathrm{o}}$ 12), à Marseille (information: M. Bonifay) et Arles (observation: C. Raynaud), en Camargue (Tréglia, 

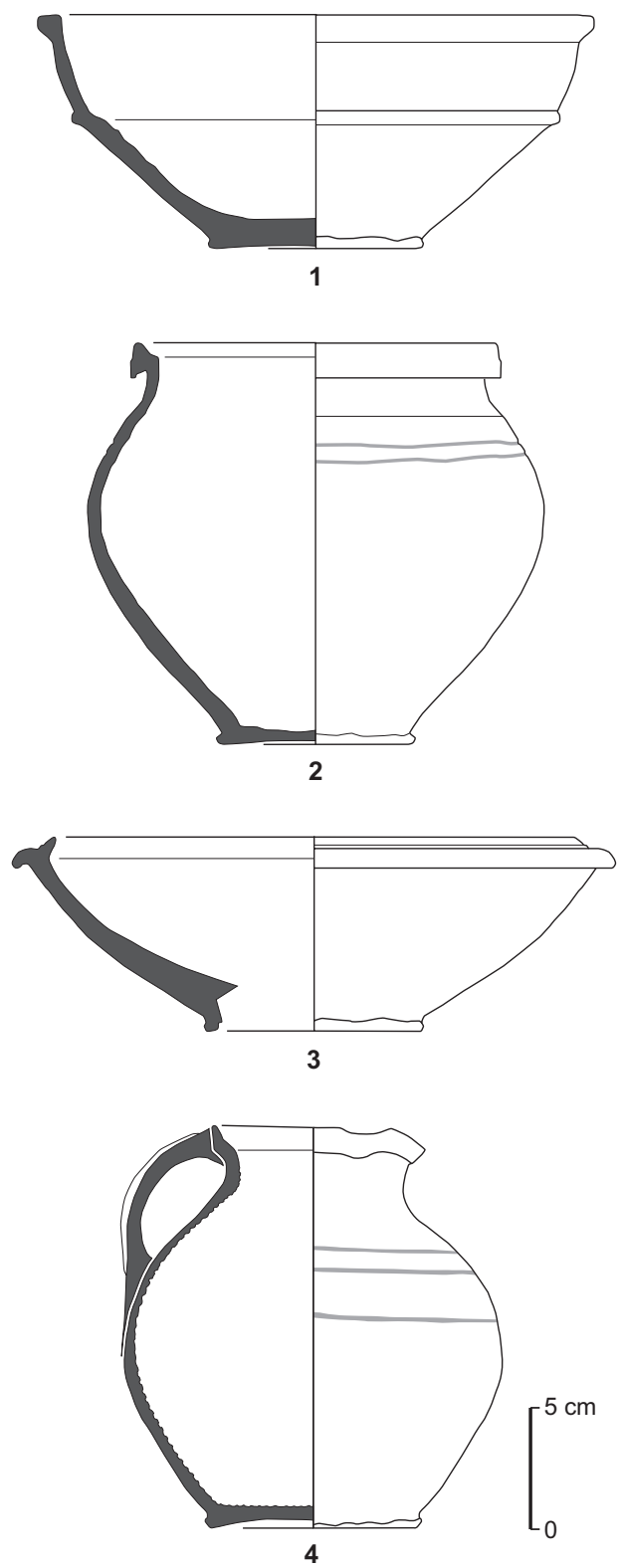

Fig. 55 - Typologie des céramiques «bistres » du Val de Saône (d'après Mani, 2004 ; DAO : M. Ott, INRAP).

2004) ainsi qu’à Maguelone (Raynaud, 2007). Sur chacun de ces sites les quantités restent faibles, mais il semble probable que la diffusion demeure sous-évaluée en raison de la méconnaissance de cette céramique jusqu'à une date récente: il serait surprenant qu'une enquête approfondie dans les collections d'Avignon ou de Nîmes ne révèle pas quelques pièces.

$\mathrm{Du} \mathrm{V}^{\mathrm{e}}$ au VIII ${ }^{\mathrm{e}}$ s., la céramique bistre connaît donc une belle longévité; sans évolution morphologique sensible, elle semble résister, au moins un temps, aux tendances décrites plus haut pour l'ensemble de la vaisselle commune à cuisson réductrice avec laquelle elle rentre progressivement en concurrence à partir du milieu du VII ${ }^{\mathrm{e}} \mathrm{s}$. Par conséquent, la céramique commune bistre, à l'inverse de la commune grise rhône-alpine, se distingue assez nettement des productions méridionales. Si tous ces produits dénotent un fonds culturel commun, la céramique bistre constitue bien une famille à part. En tout état de cause, l'hypothèse de la diffusion jusqu'au littoral méditerranéen de ces productions saônoises, dont l'origine est établie par des analyses physico-chimiques (Mani, 2005), revêt un intérêt particulier. Par ces documents, s'affirme la réalité d'une intense circulation dans le sillon Rhône-Saône, seulement soupçonnée jusqu'à présent à travers des mentions textuelles et quelques amphores égrenées jusqu'en Bourgogne. Données ténues, toujours discutables, mais qui prennent un éclairage singulier dès lors que l'on peut étoffer le dossier avec des céramiques communes, mieux diffusées et plus fréquemment attestées et offrant, de ce fait, une possibilité de cartographier ces échanges, d'une ampleur mésestimée à ce jour. Plus largement s'ouvre la perspective d'un décloisonnement des recherches régionales en insérant les régions méditerranéennes dans les courants qui irriguent la Gaule mérovingienne. Dans cette perspective, la céramique bistre du Val de Saône acquiert le rang de document majeur pour l'archéologie de la période mérovingienne dans la moitié orientale des Gaules.

C. R. et E. B.

\section{DES APPORTS, UNE INFLUENCE FRANQUE?}

Aux VI ${ }^{\mathrm{e}}$ et VII ${ }^{\mathrm{e}}$ s., l'irruption en Gaule méditerranéenne de céramiques produites en Bourgogne repose la question des influences ou des apports liés au phénomène des « grandes invasions ». En première approche, il faut relever la coïncidence de cette diffusion, très succinctement jalonnée, avec l'extension du royaume burgonde au début $\mathrm{du} \mathrm{VI}^{\mathrm{e}} \mathrm{s}$., auquel il faut ajouter le littoral de Marseille à Maguelone. On connaît les limites de ce type de superposition d'une aire de répartition archéologique sur la carte politique: que nous apprend-il hormis l'imprécision d'une cartographie autorisant toutes les extrapolations? D’un tel télescopage, retiendra-t-on l'idée d'une relation entre la mainmise burgonde et les flux commerciaux ? Notons en premier lieu que, si elle fut produite au cœur du royaume des Burgondes, la céramique bistre ne doit rien à la «culture » burgonde mais perpétue au contraire une technologie et une typologie 
ancrées dans les traditions gallo-romaines, dans un répertoire dont j'ai montré l'ubiquité en Gaule méridionale. Seuls peut-être les vases biconiques, si caractéristiques des faciès mérovingiens, pourraient témoigner d'une « ambiance germanique », encore qu'il n'est guère difficile de leur trouver une certaine parenté avec des productions gauloises comme la céramique estampée DS.P. dont la forme 18 présente aussi un profil biconique. Quant au courant d'échange, sa nouveauté ne tient qu'au sens de la diffusion si l'on se rappelle que l'axe Rhône-Saône formait, depuis des siècles, un axe de pénétration. Si l'on connaît surtout l'exemple des céramiques méditerranéennes gagnant le nord, on doit bien penser que les transporteurs chargés de cette diffusion ne rentraient pas à vide. En regard de la fréquence des arrivages de sigillée de type Claire B d'abord, puis de la Luisante des ateliers savoyards dans le quart sud-est de la Gaule, la diffusion de la céramique bistre ne fait qu'introduire un petit saut d'échelle. Était-ce même une première alors que l'on soupçonne une origine bourguignonne (de Gueugnon ?) pour les céramiques à engobe micacé identifiées à Lunel-Viel (Raynaud dir., 1990, p. 229), Arles et Lattes dès le $\mathrm{II}^{\mathrm{e}} \mathrm{s}$. ? Insidieusement, la question des courants commerciaux tardifs invite donc à s'interroger sur les silences et les lacunes de la céramologie des époques antérieures, que l'on aurait tort de supposer exemptes de zones d'ombre.

Ces considérations n'autorisent pas pour autant à éluder la question initiale, une question posée dès 1965 par S. Gagnière lorsqu'il étudiait le mobilier funéraire de la basse vallée du Rhône. Cet auteur établissait un parallèle entre les pots rhodaniens, dits carolingiens car ils étaient alors datés des VIII ${ }^{\mathrm{e}}-\mathrm{IX}^{\mathrm{e}} \mathrm{s}$., et les pots à bec connus à pareille date dans le monde franc, qu'il proposait de considérer comme les prototypes des productions méridionales (Gagnière, 1965, p. 94-97). L'idée s'imposait au sein d'un courant historiographique très influencé par les interprétations ethnicistes et par une vision « massive » des invasions qui auraient introduit des changements brutaux dans les cadres de vie. L'hypothèse cependant demeurait fragile, étayée seulement par des considérations morphotypologiques, par des comparaisons à grande distance d'Avignon à Bâle, ainsi que sur une chronologie incertaine, depuis contredite par des données qui placent la production des pégaus aux X $\mathrm{e}^{\mathrm{e}}$-XII ${ }^{\mathrm{e}}$ s. (CATHMA, 1993, p. 107).

C'est du Sud-Ouest que vinrent les éléments permettant de rouvrir le dossier, grâce au développement de l'archéologie préventive. À Rodez d'abord, le dernier niveau d'occupation d'une boutique du forum livrait, associés à des "productions locales caractéristiques des VI ${ }^{\mathrm{e}}-\mathrm{VII}{ }^{\mathrm{e}} \mathrm{s}$. " une dizaine de fragments appartenant à des vases biconiques à décor estampé de rosettes, en céramique tournée à pâte fine gris-beige à dégraissant de mica blanc, couverte noire interne et polissage externe. Des parallèles étaient établis avec du mobilier de la région rhénane (Boudartchouk, Llech, 1993), que les pièces comparables par la forme et le décor sont plus nombreuses encore en Gaule du Nord, par exemple dans les nécropoles de Vron ou Nouvion (Seillier, 1986, fig. 10, n 5), et que l'épiderme noir interne est obtenu généralement par enfumage en fin de cuisson, plutôt que par ajout d'une « couverte».

Parallèlement, plusieurs pots biconiques ainsi qu'un pot ovoïde à bec tubulaire étaient mis au jour dans des tombes $\mathrm{du} \mathrm{VI}^{\mathrm{e}} \mathrm{s}$. de la nécropole "franque " de l'Isle-Jourdain, $35 \mathrm{~km}$ à l'ouest de Toulouse (Bach, Boudartchouk, 1998, p. 217). Ce lot se singularise du précédent par sa typologie qui, bien que de "faciès mérovingien", emprunte à la tradition gallo-romaine la présence d'un pied élargi et d'un fond plat, ce qui distingue les pots biconiques de leurs voisins nordiques, sans pied et à fond creux. On ne peut donc décider s'il s'agit d'importations rhénanes ou bien d'imitations régionales, ou encore d'une évolution typologique «sous influence », les bols carénés n'étant pas absents des productions méridionales, en particulier en poterie kaolinitique (fig. 73, no 4). Si l'analyse reste à affiner afin de préciser l'origine de ces poteries, leur association dans les tombes avec un mobilier d'armement et de parure ne laisse guère de doute quant à leur attribution à une population d'origine germanique. Malgré son caractère insolite, cette découverte ne doit pas étonner outre mesure dans une région où les textes stipulent la présence de contingents réguliers de population wisigothique puis franque.

De telles données invitent à reposer certaines questions. En premier lieu, s'agit-il de cas exceptionnels ou bien des premiers éléments d'une réalité encore mal cernée? Si la première piste semble la plus probable en regard de la multiplication des fouilles, la faiblesse des études céramologiques dans le Sud-Ouest invite toutefois à conserver une marge d'appréciation à l'égard du phénomène. On ne peut invoquer le même argument dans le Sud-Est où un retour critique sur l'importante documentation disponible livre bien peu d'indices probants d'une «influence » germanique : je n'ai rien trouvé à Marseille, ni à Saint-Blaise ni à Lunel-Viel, mais en cherchant bien j'ai déniché deux pots carénés parmi le mobilier de l'habitat perché de Lombren à Vénéjan, au VI ${ }^{\mathrm{e}} \mathrm{s}$. S'il s'agit de poteries kaolinitiques tout à fait banales dans la région, en revanche la forme, avec un col incurvé et un bec tubulaire détaché du bord, affiche une singularité sans 
antécédent régional (CATHMA, 1993, fig. 4, nº 65 et fig. 5 , $\left.n^{\circ} 97\right)$. Voilà qui laisse la question ouverte et permet d'en poser une seconde: la céramique peut-elle être utilisée comme révélatrice d'une identité ethnique ou culturelle ? L'exemple précédent de la céramique bistre incite à un certain recul à l'égard de cette lecture de documents qui demeurent en eux-mêmes peu probants et qu'il faudrait replacer dans une perspective d'ensemble de la culture matérielle et technique avec, outre la céramique, le mobilier de parure, les formes d'habitat, les techniques de construction comme les fonds de cabane, etc. Après des décennies de «méditerranéocentrisme », il nous incombe désormais d'accepter l'idée d'un métissage nord-sud et d'en repérer les signes.

C. R.

\section{LES PRODUCTIONS DE VAISSELLE FINE}

\section{ATELIERS ET PRODUCTIONS}

Les deux notions d'atelier et de production se trouvent généralement confondues ou employées l'une pour l'autre par les archéologues, autant par convenance que par ignorance de la variabilité des différentes productions au sein d'un même atelier. Il y a donc lieu de poser des définitions exemptes d'ambiguité. La notion d'atelier repose à la fois sur l'unité du lieu et sur la chaîne opératoire. Elle désigne par conséquent le site où sont établies les différentes installations techniques permettant la fabrication de produits de terre cuite, poteries et/ou terres architecturales. Rien n'autorise donc à confondre l'atelier en tant qu'ensemble opératoire avec la ou les productions qui en sont issues. Une production céramique peut être définie comme le résultat d'une série de choix techniques et morphologiques opérés en fonction d'une commande et d'impératifs fonctionnels. En ce sens, un atelier peut fournir, à la demande, une ou plusieurs productions : de la vaisselle à cuire, de la vaisselle pour le service, des terres cuites architecturales ou des récipients de transport, des lampes. Le développement des fouilles d'officines de potiers montre à quel point ces centres artisanaux s'adaptent à une demande en diversifiant leurs productions. On comprend donc dans ces conditions la difficulté inhérente à l'analyse céramologique qui, sans toujours disposer d'éléments d'identification précise de tel ou tel atelier, doit tenir compte à la fois du fait qu'une production spécifique peut provenir - et provient en effet le plus souvent - de plusieurs ateliers distincts, tandis qu'un même atelier peut fournir des produits fort différents, qu'il n'est pas toujours possible de rattacher à une origine commune.
Pour l'Antiquité tardive, les ateliers méridionaux soulignent cette ambivalence de la notion d'atelier, par exemple à Générac (Raynaud, 1982) où l'on a produit au dernier quart du IV ${ }^{\mathrm{e}} \mathrm{s}$. (les contextes d'Afrique ayant livré des lampes analogues laissent envisager une date plus tardive, vers le milieu du V ${ }^{\mathrm{e}}$ s., voir Bonifay, 2004, p. 266), à partir d'une argile calcaire commune à toutes les pièces, une vaisselle engobée à décor estampé du type DS.P., des lampes à huile engobées imitant des modèles africains et enfin une vaisselle commune d'ollae dépourvues d'engobe (fig. 56). À la même date les produits de l'atelier de la Quintarié à Clermontl'Hérault apparaissent plus contrastés, se partageant entre une production de vaisselle fine de DS.P. à pâte calcaire, et une poterie commune à pâte sableuse, les deux séries possédant des répertoires bien distincts (fig. 56) (Barberan, Pomarèdes, 1991). En Savoie, l'atelier de Portout, fameux pour sa production de vaisselle «Luisante", semble au contraire obéir à une forte spécialisation, car il a livré exclusivement de la vaisselle de table, mais il s'agit seulement d'un élément au sein d'un ensemble d'officines, comme le laisse penser la découverte d'une production analogue sur le site voisin de Conjux (Pernon, Pernon dir., 1990). C'est aussi à une production homogène que semblent voués les différents ateliers de céramique commune à pisolithes de la région nîmoise, mais en ce cas l'absence de données de fouille invite à la prudence. Dans la partie rhodanienne de la cité nîmoise, c'est encore une production très restreinte que l'on identifie à Roquemaure où un four unique était établi au $\mathrm{V}^{\mathrm{e}} \mathrm{s}$. dans les bâtiments à l'abandon d'un centre domanial et produisait une vaisselle commune kaolinitique (CAG, 30/3, p. 541).

Il reste beaucoup à faire pour caractériser le cadre technique et l'organisation des ateliers de l'Antiquité tardive. Si les ateliers du Sud-Est que je viens de citer se trouvent épars à la campagne, c'est en revanche en ville que l'on trouve les ateliers du Toulousain et de Novempopulanie, à Toulouse, Eauze et Lectoure assurément, mais probablement aussi à Auch et à Cahors où les ateliers ne sont toutefois pas encore localisés (Dieulafait et al., 1996). En Aquitaine, à l'autre extrémité de notre enquête, c'est à la campagne qu'était produite la vaisselle commune, notamment par l'atelier de Martinière (Robin, Chambon, 2002).

C. R.

\section{CÉRAMIQUE LUISANTE}

Depuis sa caractérisation et sa première typologie au début des années 1960 (Lamboglia, 1963), la céramique «Luisante " a fait l'objet de plusieurs mises au point faisant 


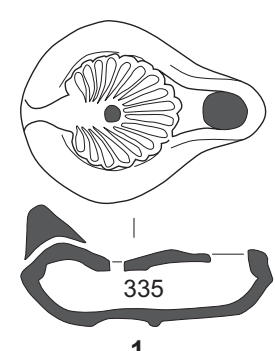

1

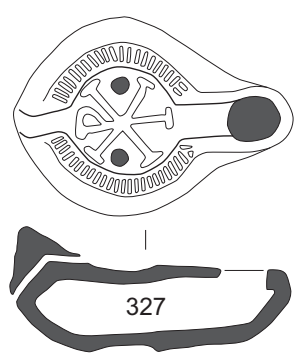

2

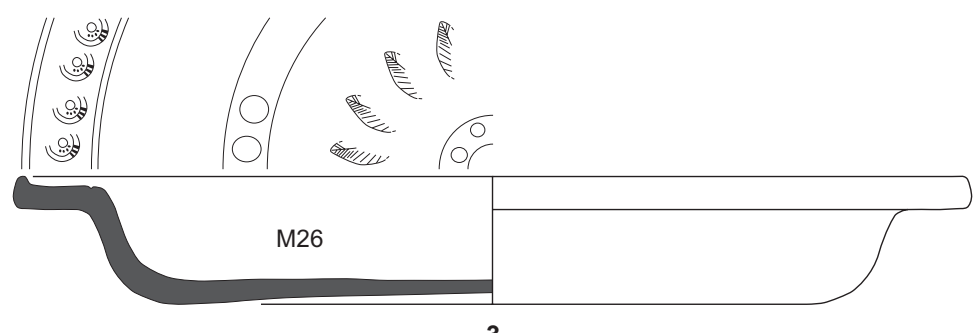

3

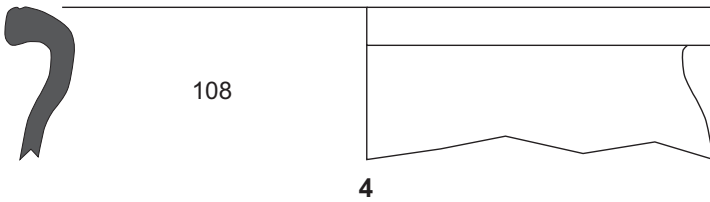

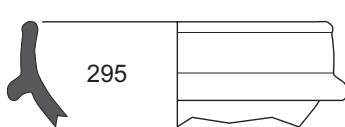

5
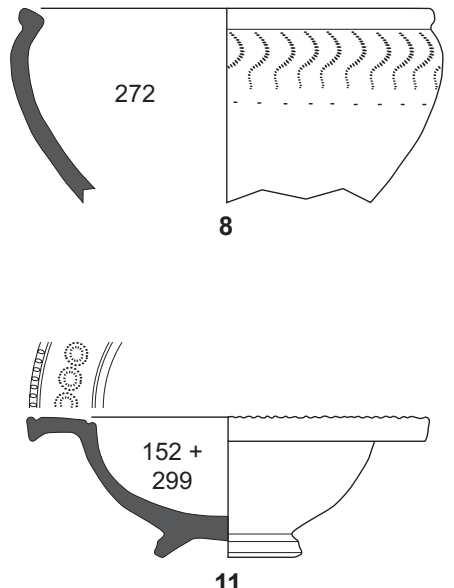

10

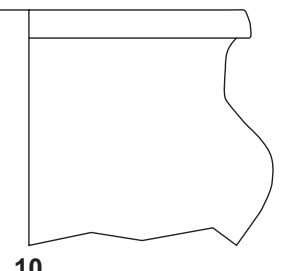

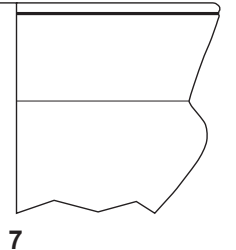

3

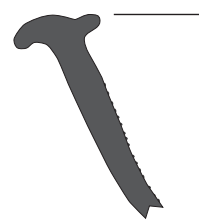

164

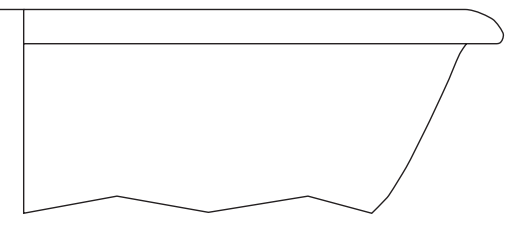

12
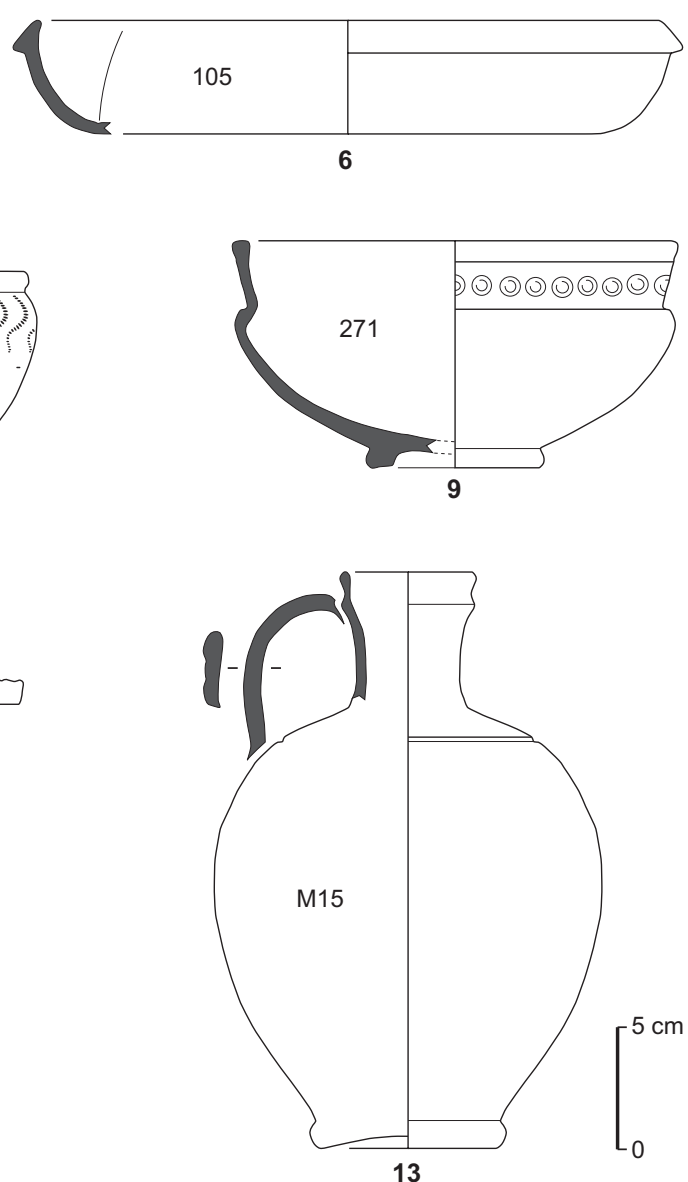

Fig. 56 - Principales productions de l'atelier de Générac (DAO : M. Ott, INRAP).

apparaître les problèmes d'identification et d'attribution, au sein de l'ample famille des "céramiques à revêtement argileux non grésé ». Ce qualificatif a été introduit à la fin des années 1970 lorsque la multiplication de la documentation rendit évidente la multiplicité des zones de production et la complexité de la diffusion, que la typologie initiale n'avait pas envisagées. En particulier, l'étude de collections de plus en plus abondantes mettait en avant l'ambiguité des qualificatifs visuels, la présence de reflets irisés ou métallescents qui justifiait le terme de "luisante " pouvant s'appliquer aussi bien à des poteries que la typologie et la chronologie invitaient à attribuer à la céramique Claire B. A contrario, certaines pièces appartenant à la typologie de la Claire B, révélaient un aspect extérieur « luisant» sans que l'examen visuel de la pâte et du revêtement argileux permette de trancher (Collectif, 1986). L'analyse physico- 
chimique apporta une clarification en établissant qu'il existait bien deux «productions » provenant de deux aires distinctes, l'une rhodanienne pour la Claire B, l'autre savoyarde pour la Luisante (Desbat, Picon, 1986, p. 14-16).

Les connaissances se sont affinées au cours des dernières années, les analyses révélant la multiplicité des ateliers producteurs, officines éparses dans la haute vallée du Rhône entre Thonon-les-Bains, Annecy, le lac du Bourget et Aoste. Tout en révélant la diversité des provenances, ces mises à jour mettent en évidence la longévité d'une production qui n'est en rien spécifique de l'Antiquité tardive mais plonge ses racines dans une activité que l'on suit désormais depuis la fin du $\mathrm{II}^{\mathrm{e}}$ s. apr. J.-C. (André, 2003). Dans la région alpine, la vallée du Rhône et les régions méditerranéennes, durant plusieurs siècles les "céramiques à revêtement argileux " ont constitué l'essentiel de la vaisselle fine de table, dans une proportion variable par rapport aux autres vaisselles fines, en particulier africaines.

Si elle n'est pas toujours facile à distinguer des productions voisines, à l'œil nu, la céramique Luisante conserve néanmoins sa singularité typochronologique et demeure l'un des fossiles directeurs des niveaux d'occupation tardoantiques, en concurrence avec la Claire B qui reste bien attestée jusqu'au $\operatorname{IV}^{\mathrm{e}} \mathrm{s}$. Dans l'établissement du Bivan à L'Albenc où la typologie couplée à des analyses physicochimiques fournit un ensemble de référence du milieu du $\mathrm{IV}^{\mathrm{e}} \mathrm{s}$., la céramique Claire B livre encore $76 \%$ des pièces de vaisselle fine tandis que la Luisante atteint seulement $20 \%$ (Ayala, 1996, p. 255).

On situe généralement l'apparition de la céramique Luisante dans la seconde moitié $\mathrm{III}^{\mathrm{e}} \mathrm{s}$., ce que confirme l'étude d'un ensemble clos découvert à Annecy et provenant probablement d'un atelier local encore non localisé (André, 2003). Cette série, qui comporte au minimum 260 pièces, emprunte une large part de son répertoire à la vaisselle sigillée (fig. 57), types Drag. 33 et Curle 15, ainsi qu'aux productions à revêtement argileux des II $^{\mathrm{e}}$ et $\mathrm{III}^{\mathrm{e}} \mathrm{s}$., gobelets ovoïdes et coupelles hémisphériques, ou encore à la Claire B en ce qui concerne le type Lamb. 2/37-Portout 27 qui reprend le type 14 en Claire B, lui-même avatar de la coupe Drag. 37 en sigillée (André, 2003, p. 124). Avec le mortier Drag. 45Portout 40, lui aussi hérité d'un répertoire antérieur, la coupe Lamb. 2/37-Portout 27 restera tout au long de la production l'une des pièces les plus communes, encore dominante au $\mathrm{V}^{\mathrm{e}} \mathrm{s}$. dans l'atelier de Portout. Sur la rive du lac du Bourget, cette officine bien connue grâce à une étude détaillée paraît vouée à la seule vaisselle Luisante mais avec deux phases distinctes, la phase principale se situant dans les premières décennies du V ${ }^{\mathrm{e}}$ s. (Pernon, Pernon dir., 1990). Cette production tardive révèle une large variété typologique avec un répertoire de 69 formes largement enrichi et renouvelé par rapport aux productions du III et du $\mathrm{IV}^{\mathrm{e}} \mathrm{s}$. Si les formes ouvertes s'inspirent encore largement du répertoire de la Claire B (types Portout 1 à 7), les coupes, cruches et gobelets révèlent par contre des emprunts à la vaisselle commune (fig. 58). Avec les types Portout 37 et 40 déjà cités, les tasses carénées 36 et les gobelets ovoïdes 65, 68 et 76 caractérisent ce faciès tardif, tant dans l'atelier savoyard que sur les sites consommateurs, jusqu'au littoral méditerranéen (Raynaud dir., 1990, p. 184 par exemple).

La fin de la production reste difficile à cerner. $\mathrm{Si}$ la céramique Luisante est régulièrement présente dans les niveaux d'habitat jusqu'au milieu du $\mathrm{V}^{\mathrm{e}} \mathrm{s}$. (Raynaud dir., 1990, p. 199-211; Ayala, 1998), elle est en revanche absente dès la fin de ce siècle et le début du siècle suivant, ce qui invite à envisager un arrêt de la production durant la seconde moitié du $\mathrm{V}^{\mathrm{e}} \mathrm{s}$., selon un rythme - brutal ou progressif - que l'on pourra apprécier lorsque les contextes de cette période seront mieux cernés.

L'aire de répartition s'avère très ample, embrassant une part de la Gaule de l'Est, la Bourgogne, le Lyonnais et la région alpine, ainsi que la Franche-Comté dans une moindre mesure. Elle s'étend largement dans toute la vallée du Rhône, le fleuve apparaissant comme le principal vecteur de la diffusion de ces ateliers riverains. Des niveaux d'occupation du quartier Saint-Jean à Lyon donnent un aperçu de la consommation, la Luisante représentant 36,5\% de la vaisselle fine au milieu du $\mathrm{IV}^{\mathrm{e}} \mathrm{s}$., puis $66 \%$ à $69 \%$ au $\mathrm{V}^{\mathrm{e}} \mathrm{s}$. (Ayala, 1998). Les chiffres s'avèrent équivalents à la campagne avec $20 \%$ de Luisante au Bivan au milieu du IV $^{\mathrm{e}}$ s. (Ayala, 1996) et $32 \%$ au V ${ }^{\mathrm{e}}$ s. à Poncin, avant de chuter à la phase suivante, dans un $\mathrm{V}^{\mathrm{e}} \mathrm{s}$. avancé (Faure-Boucharlat dir., 2001, p. 153). Dans la villa du Vernai à Saint-Romainde-Jalionas, au début du IV ${ }^{\mathrm{e}} \mathrm{s}$., la Luisante représente $16 \%$ à $18 \%$ de la vaisselle fine (Laroche, Théolas, 2003). À Châteld'Arrufens (canton de Vaud), la Luisante fournit $93 \%$ de la vaisselle fine au dernier quart du IV ${ }^{\mathrm{e}} \mathrm{s}$. et au premier quart du $\mathrm{V}^{\mathrm{e}}$ s. (David-Elbiali, Paunier dir., 2002). Simples indicateurs de tendances qui mériteraient des confrontations approfondies, ces chiffres convergent cependant pour souligner la forte progression de la consommation, qui double son score $\mathrm{au} \mathrm{V}^{\mathrm{e}} \mathrm{s}$. avant de décliner vers la fin de ce siècle.

La diffusion se poursuit jusqu'au littoral méditerranéen, mais un contraste oppose les deux rives du fleuve, la rive gauche connaissant une fréquence inférieure de moitié ou des deux tiers par rapport aux volumes attestés sur la rive 

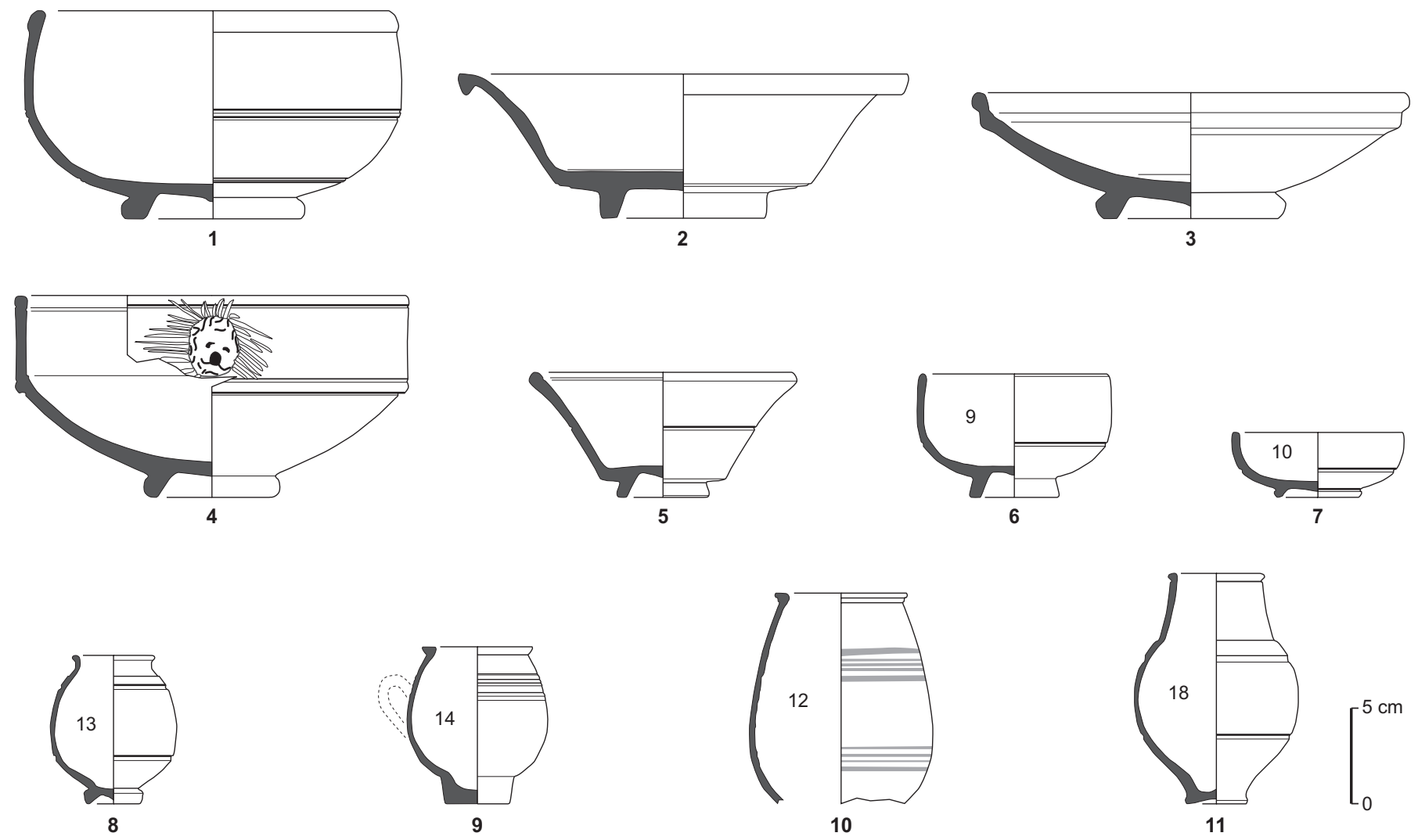

Fig. 57 - Typologie des céramiques « à revêtement argileux » d'Annecy (d'après André, 2003) (DAO : M. Ott, INRAP).

droite. En Languedoc, le village de Lunel-Viel permet de suivre l'évolution de la Luisante, qui représente (avec une part d'incertitude due à quelques Claires $\mathrm{B}$ résiduelles qui n'ont pu être discriminées), $68 \%$ à $73 \%$ de la vaisselle fine aux IV ${ }^{\mathrm{e}}$ et $\mathrm{V}^{\mathrm{e}}$ s. (Raynaud dir., 1990, p. 253-254). Même proportion à Nîmes où la Luisante s'établit entre $64 \%$ et $77 \%$ de la vaisselle fine au $\mathrm{IV}^{\mathrm{e}} \mathrm{s}$. (Monteil, 1993, p. 170-185). La densité reste forte vers l'ouest, notamment dans le bassin de Thau, autour de la villa de Loupian (Pellecuer, 1983), puis faiblit à Narbonne où elle apparaît minoritaire au début du $\mathrm{V}^{\mathrm{e}}$ s., sans que l'on dispose encore de données nécessaires aux comparaisons (Solier dir., 1991 ; Ginouvez dir., 19961997). Vers l'ouest, la diffusion faiblit encore et devient difficile à identifier, par exemple à Rodez et à Toulouse où l'on envisage plutôt une production régionale imitant certaines formes de la vaisselle Luisante (Boudartchouk, Llech, 1993, p. 156 ; Dieulafait et al., 1996, p. 273).

Vers le sud, la céramique Luisante est présente dans les Corbières, à Tuchan, où sa proportion descend à $5 \%$ de la vaisselle fine (Térès, Rigoir, 2001, p. 91). Elle se diffuse au-delà de la province de Narbonnaise, restant bien attestée sur de nombreux sites du nord de la Tarraconnaise (Aicart i Hereu et al., 1991), notamment à Iluro/Mataro où la Luisante représente $10 \%$ de la vaisselle fine dans la seconde moitié du $\mathrm{V}^{\mathrm{e}} \mathrm{s}$. et le début du $\mathrm{VI}^{\mathrm{e}} \mathrm{s}$., et encore $4 \%$ à $5 \%$ au $\mathrm{VI}^{\mathrm{e}} \mathrm{s}$, , ce qui pose le problème de la représentativité de ce mobilier, le plus tardif actuellement connu : doit-on penser à des éléments résiduels (Cela Espin, Revilla Calvo, 2004, p. 365) ? La province de Bétique connaît aussi des arrivages mais en proportion infime (Bourgeois, Mayet, 1991, p. 368369), de même que la Sardaigne (Villedieu, 1984, p. 232) et l'Afrique du Nord (Bonifay et al., 2002-2003).

Sans être négligeable, la diffusion s'avère plus faible à l'est du Rhône où les sites provençaux révèlent des pourcentages bien inférieurs à ceux du Languedoc: $11 \%$ de la vaisselle fine à Arles (Congès, Leguilloux, 1991, p. 212), $20 \%$ à Aix-en-Provence (mais dans des niveaux du $\mathrm{VI}^{\mathrm{e}} \mathrm{s}$.), $21 \%$ à Saint-Julien-les-Martigues au v ${ }^{\mathrm{e}} \mathrm{s}$. et seulement 3,5\% à Marseille-Bourse dans des niveaux de la fin du $\mathrm{V}^{\mathrm{e}} \mathrm{s}$. et du début du $\mathrm{VI}^{\mathrm{e}} \mathrm{s}$. (CATHMA, 1986, p. 39). La diffusion se poursuit vers l'est, sur la Côte d'Azur où les Claires B et les Luisantes représentent ensemble de $5 \%$ à $15 \%$ de la vaisselle fine aux IV et $V^{\mathrm{e}}$ s. (Pellegrino, 2001, p. 446), et sur le littoral ligure où les fouilles de Vintimille et d'Albenga 

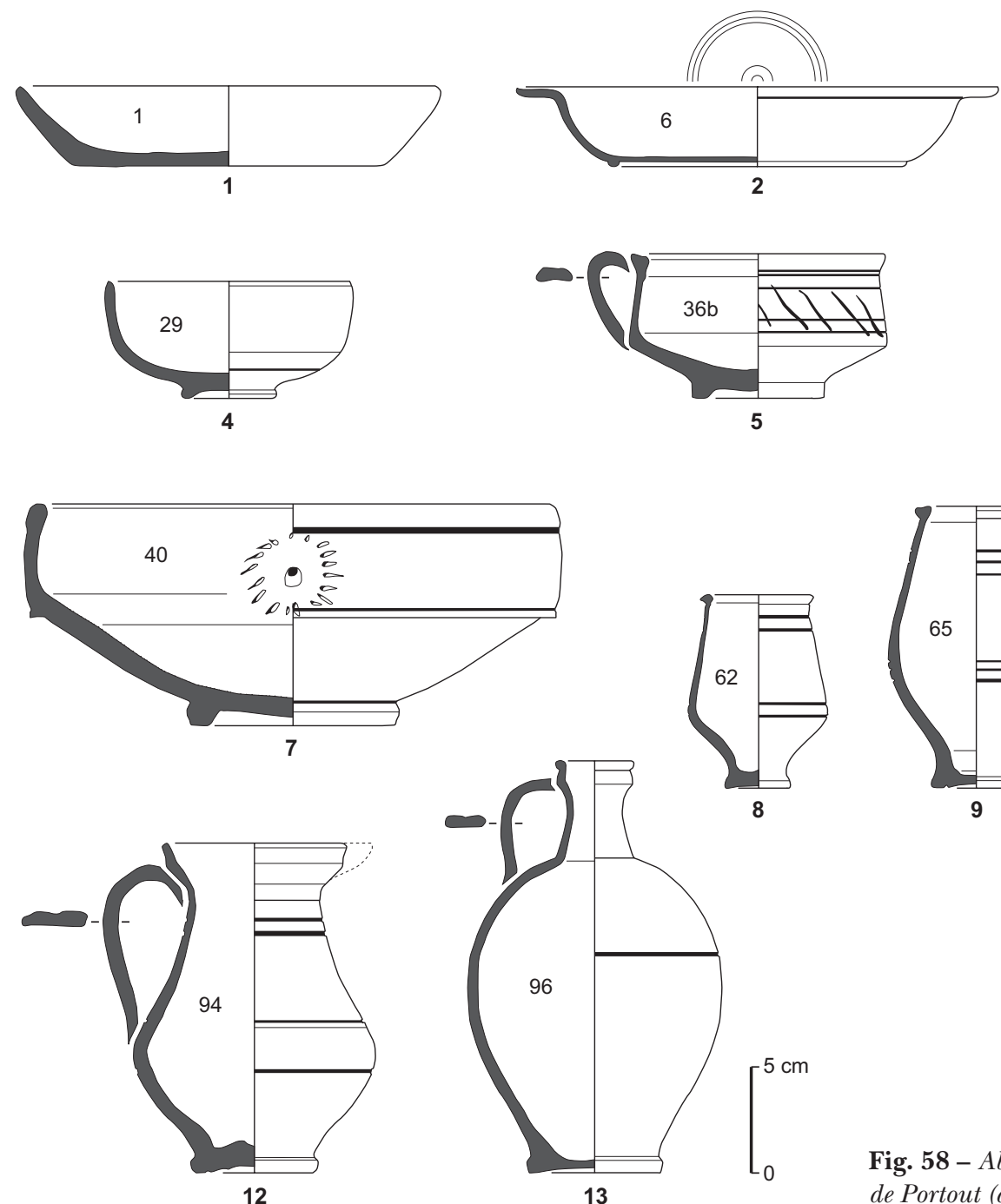

avaient donné à N. Lamboglia les premiers matériaux de son étude typochronologique (Lamboglia, 1963, p. 166). Sans menacer l'hégémonie africaine sur le commerce de la vaisselle fine en Méditerranée occidentale, la vaisselle Luisante prend donc néanmoins place parmi les produits de grande diffusion, à l'échelle de l'économie de l'Empire d'Occident.

C. R.

\section{LES DÉRIVÉES-DES-SIGILLÉES PALÉOCHRÉTIENNES (DS.P.)}

Dans les deux dernières décennies, de nombreuses découvertes, de dimensions variées mais toutes d'un intérêt certain, ont été signalées, précisant la mise en place géographique et chronologique des sigillées tardives de Gaule.
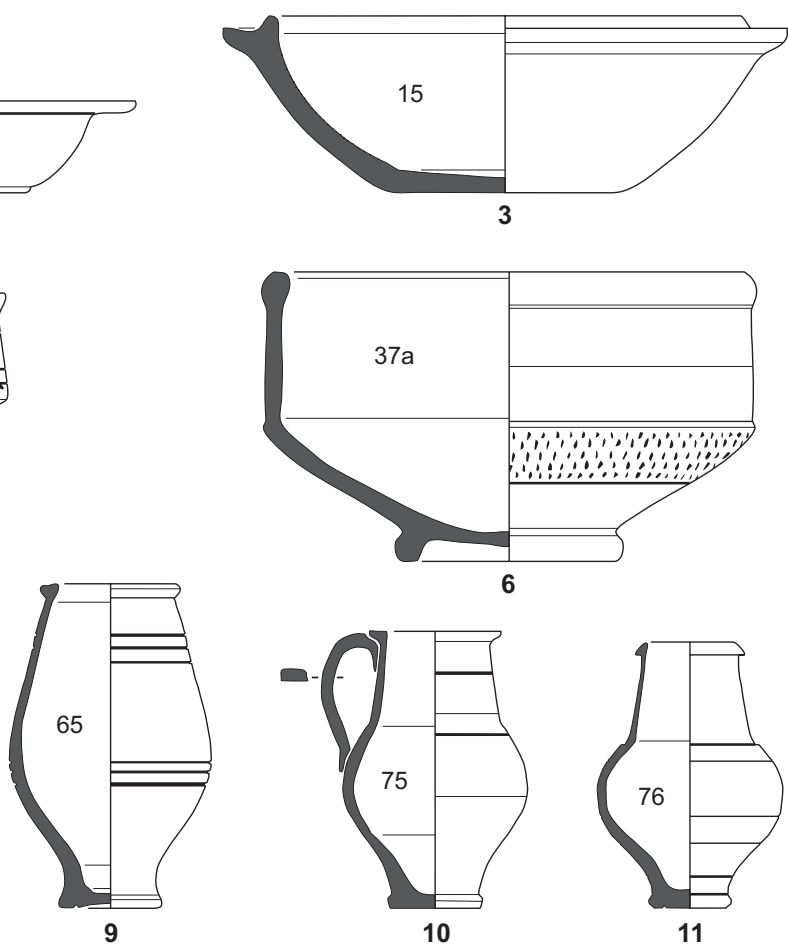

Fig. 58 - Abrégé typologique des céramiques Luisantes de l'atelier de Portout (d'après Pernon, Pernon dir., 1990).

Les plus importantes furent, pour la détermination du groupe Languedocien, la fouille de la basilique de la Lombarde à Narbonne et, pour la mise en place de la chronologie de la production marseillaise, les nombreuses fouilles locales. D'autres découvertes, quantitativement secondaires, ont permis de moduler les caractéristiques de chaque famille.

Les différents groupes ou familles des DS.P. stylistiquement et technologiquement déterminés se répartissent suivant des aires que l'on peut considérer comme " géo-logiques".

Le groupe Atlantique alimente les plaines de l'Ouest, le groupe Languedocien occupe le couloir formé par les Pyrénées et le Massif central ainsi que leurs contreforts, le groupe Provençal pénètre depuis la Méditerranée le long de la vallée du Rhône. Les limites de ces régions correspondent 
assez précisément à ce qui seront par la suite les frontières des royaumes «barbares ».

L'homogénéité du groupe Atlantique et la position centrale de Bordeaux, attestées par une répartition homogène et les catalogues restreints des formes et des poinçons, paraissent se confirmer (Soulas, 2000).

Les caractéristiques du groupe Languedocien, dont la principale est l'utilisation de la postcuisson oxydante parallèlement à la cuisson réductrice, se trouvent principalement développées par les nombreux documents narbonnais (Rigoir, Rigoir, 1991). Des ateliers secondaires se signalent par des structures sur le site de la Quintarié à Clermontl'Hérault (Barberan-Pomarèdes, 1991) et quelques particularités révèlent des ateliers satellites dans la région de Carcassonne (Courtieu et al., 1980), de Béziers ou de Rodez (Bourgeois, 1993).

La disposition des empreintes en couronne sur les fonds d'assiettes, le profil des formes à bords en amande, la postcuisson oxydante témoignent d'analogies certaines avec les sigillées africaines, de même que la datation haute des produits languedociens, et peuvent laisser entrevoir un emprunt direct, sinon une réelle filiation?

Le groupe Provençal est éclaté en multiples ateliers. En Suisse, la cuisson réductrice est généralisée, le gris est assez sombre. Les formes sont lourdes et les parois épaisses. En descendant le Rhône, il est plus difficile de cerner des productions, malgré l'existence de l'atelier des Putiers/Amour de Dieu à Soyons en Ardèche (cf. réunion CATHMA du 20 janvier 1989).

À Valence et sur le site de Mars à Saint-Georges-les-Bains (Durand-Rigoir, 2001), on rencontre un assez grand nombre de formes 56, formes locales d'assiettes à bord simple rabattu à angle aigu vers l'intérieur, accompagnées d'un bol caréné et d'une f.18. Leur particularité est une pâte d'un gris verdâtre recouverte d'un engobe qui se desquame en minuscules écailles. Dans le Vaucluse, les fouilles d'Apt ont fourni un matériel aux caractéristiques propres, dans les profils et le répertoire des poinçons (Kauffmann et al., 1987).

Les caractères languedociens influencent quelques fabrications aux alentours du delta du Rhône, ce que l'on peut constater par exemple à Avignon, mais surtout grâce à la découverte sur le site de Saint-Julien-lès-Martigues (Rigoir et al., 1985, p. 97) d'un lot typologiquement distinct des produits régionaux (par la postcuisson oxydante entre autres), mais cependant distincts des normes languedociennes. Les fouilles des thermes à Aix-en-Provence ont livré un certain nombre de fragments identiques (cf. réunion CATHMA du 17 février 1994).
Le répertoire marseillais est le plus disparate, bien que certaines particularités de profil et de décor autorisent des attributions certaines (Rigoir, Rigoir, 1997 ; Bonifay et al. dir., 1998 ; Mukaï, 2001). Les multiples et importantes fouilles de la ville ont surtout permis d'apporter des précisions chronologiques (voir T. Mukai, notice 7, infra, p. 159-161).

\section{DIFFUSION}

Alors que les ateliers languedociens et marseillais ont exporté tout autour de la Méditerranée, on ne connaît aucune trace de produits bordelais sur les côtes espagnoles ou portugaises. Seuls quelques objets sont signalés en Angleterre. Les deux autres officines se concurrencent, principalement en Catalogne. Leurs poteries se retrouvent ponctuellement dispersées, languedociennes en Corse ou en Grèce, marseillaises sur l'île d'Elbe ou au Maghreb.

Un commerce croisé à proximité des limites de chaque groupe fournit des clientèles communes, bordelais et languedociens fournissent Agen (CATHMA, 1993) et le Gers (Lapart et al., 1986), languedociens et marseillais dans l'Hérault, mais principalement sur la côte, le fleuve faisant frontière dans les terres.

Remarque plus anecdotique, des tessons isolés se retrouvent à bonne distance de leur origine. À Bordeaux, quelques fragments languedociens ont été trouvés ainsi qu'une coupelle de f. 2 marseillaise, un poinçon de même origine, un seul, à Narbonne. Deux poinçons bordelais sont pointés à Toulouse (CATHMA, 1993), deux autres, languedociens, se retrouvent l'un à Digne-les-Bains (Rigoir, 1995), l'autre dans l'établissement rural de Saint-Pierre-de-Vence à Eyguières (Rigoir et al., 2001, p. 73), mais cela est moins insolite, ce site révélant de nombreux documents jamais signalés auparavant, dont le plus bel exemple est la copie d'une palmette animalière d'origine atlantique! (fig. 59).
1

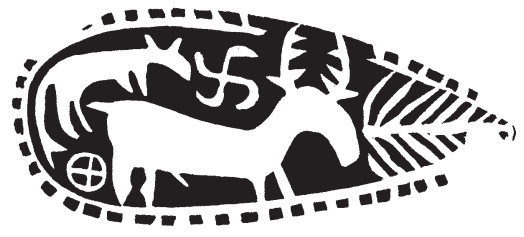

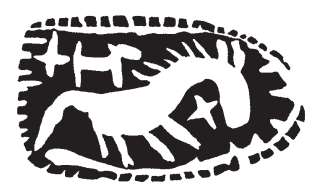

2
Fig. 59 - Dérivée-de-Sigillées Paléochrétiennes : 1, le poincon 894 du groupe Atlantique trouvé à Poitiers, Nantes et Blois ; 2, sa copie 4408 apparue à Eyguières, Saint-Pierre-de-Vence (DAO : Y. Rigoir, LAMM). 


\section{FORMES}

Si les différents profils des vases sont utilisés pour en distinguer les catégories, leur rôle évident est aussi de faire entrevoir leur utilité dans l'organisation des repas. L'utilisation de certaines formes creuses est difficile à préciser: il est impossible de boire dans les bols à marli, f. 3, de contenance assez petite (un quart à un tiers de litre) et le bord resserré du profil type de la forme 6 languedocienne exclut aussi de la porter aux lèvres ; le bord des f. 6 atlantiques, en revanche, est simple et affiné et leur contenance varie entre 0.3 et 2,6 litres. Les diverses f. 15 et 18 sont d'un gabarit équivalent, légèrement supérieur, comme celui des f. 16 atlantiques. Certaines exceptions peuvent recevoir 10 litres de liquide, ce qui donne à ces divers récipients des utilisations très diverses.

Un intéressant échange de vues sur une question de vocabulaire a amené à nuancer les termes évoquant l'utilisation des différentes formes plates suivant leur dimension.

Les graphiques dressés pour les diamètres mettent ainsi en évidence la possibilité de différencier assiettes, plats ou plateaux pour les formes 1 , à marli, 4 , à bord simple et 8 , à bord en amande (fig. 60).

En Provence, le diamètre d'une majorité d'objets de formes 1 et 4 se cantonne entre $20 \mathrm{~cm}$ et $30 \mathrm{~cm}$ autour d'un pic nettement marqué ; les f. 8 se répartissent plus régulièrement dans la même fourchette.

Dans le Languedoc, où la forme 4 est absente, le même pic se remarque pour les autres formes mais une série orangée s'étale entre $40 \mathrm{~cm}$ et $60 \mathrm{~cm}$.

Les formes 1 et 4 de la production atlantique présentent des physionomies à peu près équivalentes, montrant chacune deux courbes nettement différenciées, particulièrement pour la f. 4, l'une de $17 \mathrm{~cm}$ à $20 \mathrm{~cm}$, l'autre de $20 \mathrm{~cm}$ à $35 \mathrm{~cm}$, renfermant des quantités comparables d'objets.

Suivant ces images, il est possible de parler de plateaux pour la série languedocienne et de constater dans l'Ouest l'utilisation individuelle ou collective d'assiettes et de plats.

\section{DÉCORS}

Le guillochage, technique rapide d'animation des surfaces, est diversement employé suivant les productions. S'il est partout présent sur les marlis, il cerne le motif central des fonds d'assiettes et recouvre entièrement certains bols des ateliers atlantiques alors qu'il sépare les différents registres sur les pièces creuses languedociennes. En Provence, il se limite à certaines parties, les cols des formes 18 par exemple.

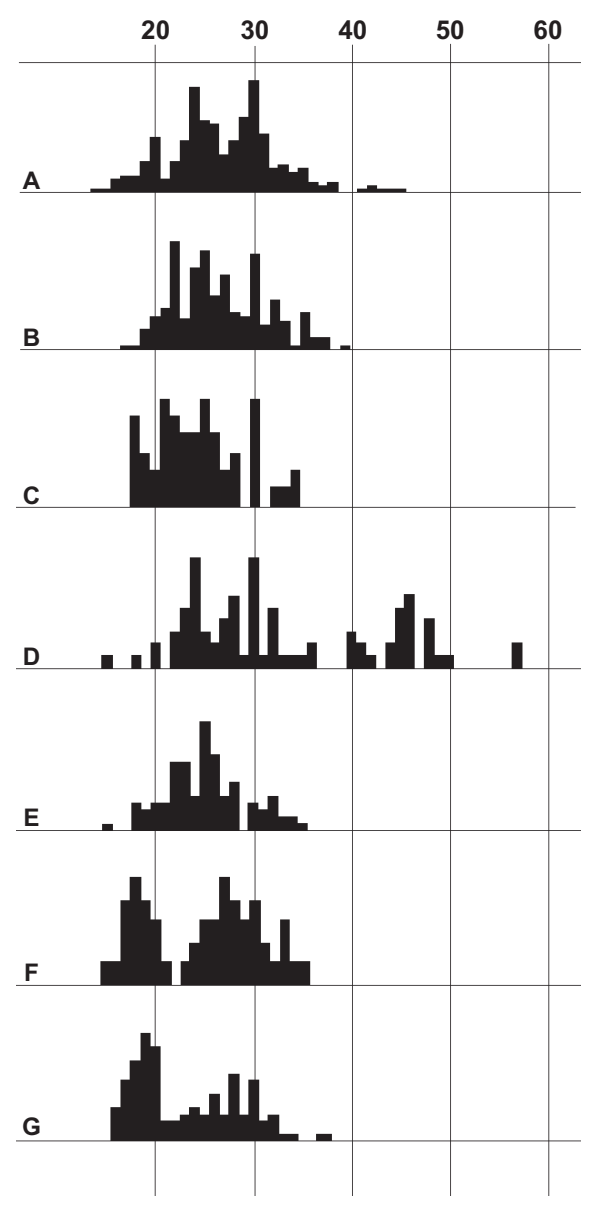

Fig. 60 - Diamètres des formes plates des Dérivées-de-Sigillées Paléochrétiennes. Groupe Provençal: A, forme 1 ; B, forme 4 ; $C$, forme 8. Groupe Languedocien: D, forme $1 ; E$, forme 8. Groupe Atlantique : F, forme 1 ; $G$, forme 4. Les barres ne représentent pas des quantités absolues, mais les rapports entre les nombres de diamètres par forme (DAO: Y. Rigoir, LAMM).

Les impressions de poinçons demeurent le moyen favori de décoration et d'expression des potiers. Les outils utilisés dans l'Ouest sont d'assez grandes dimensions, $4 \mathrm{~cm}$ pour les rouelles, $6-7 \mathrm{~cm}$ pour les palmettes. Ils sont généralement soigneusement gravés et manipulés, ce qui en facilite l'identification. Leur petit nombre, un dixième du répertoire général, renforce encore l'homogénéité de cette production. Les palmettes, dont les nervures sont généralement curvilignes, sont disposées suivant les rayons des surfaces circulaires et à la verticale des parois des bols. À l'inverse, les poinçons utilisés par les ateliers languedociens sont petits, nombreux et leur impression est souvent négligée. Ils forment des couronnes concentriques sur les fonds et leurs dimensions autorisent des registres super- 


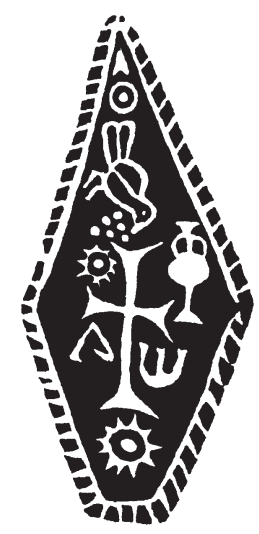

Fig. 61 -Dérivée-de-Sigillées Paléochrétiennes: poincon 3384 attesté à Saint-Blaise, Olbia et complet à Roses (Catalogne) (DAO : Y. Rigoir, LAMM).

posés sur les parois des bols. Aucune règle ne paraît se dégager du catalogue provençal où nervures et hachures sont rectilignes et parallèles.

Le catalogue des poinçons augmente, 5479 aujourd'hui, dans un même répertoire de motifs qui peuvent être soit géométriques, soit figuratifs. Les premiers sont divisés par des pointillés, des hachures, des pastilles : rouelles et palmettes pour les deux tiers d'entre eux, carrés, rectangles et figures diverses pour presque tous les autres. Les représentations animales: cerfs, chiens, agneaux, colombes, poissons se retrouvent associés, parfois accompagnés de silhouettes sommaires ; un personnage est mieux dessiné à Marseille et des masques barbus sont encadrés d'arcatures sur les vases languedociens. Des signes ou symboles : croix, chrismes, étoiles, lunes ou «sceaux de Salomon » complètent ce répertoire pour lesquels le terme de «décors" ne semble alors pas vraiment approprié, mais l'iconographie potière, d'Aphrodite à Hercule, ne fut que rarement laïque ou apolitique. Un récent exemple en est donné par un poinçon dont des fragments avaient été découvert à SaintBlaise et à Olbia (Hyères). Ses impressions rayonnantes sur un fond d'assiette de la citadelle de Roses en Catalogne (Llinas et al., 1997) montrent dans un grand losange une combinaison rassemblant croix monogrammatique, oiseau, vase, raisin, soleil, étoile et lune (fig. 61).

Y. R.

\section{LES AUTRES PRODUCTIONS}

\section{LES LAMPES}

Les lampes africaines des $\mathrm{V}^{\mathrm{e}}-\mathrm{VII}{ }^{\mathrm{e}} \mathrm{s}$, de type Atlante X, ont été fréquemment imitées dans tout le bassin méditerranéen. Le midi de la Gaule ne fait pas exception à cette tendance. On citera deux exemples. Marseille, tout d'abord,
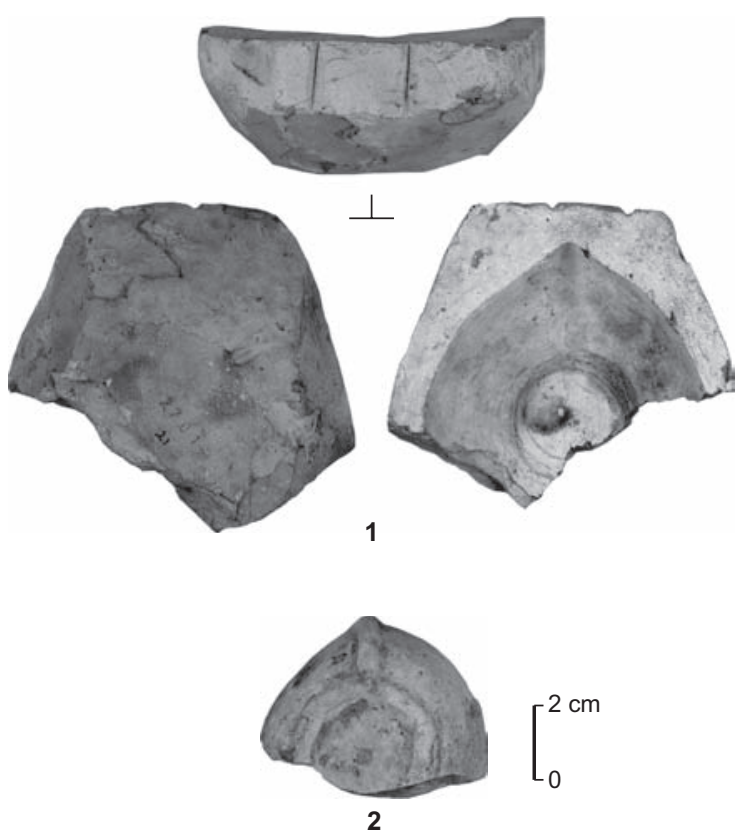

Fig. 62 - Imitations de lampes africaines provenant de Marseille, la Bourse (d'après Bonifay, 2004) (DAO : M. Bonifay, CNRS).

où la production de lampes de type africain est attestée par la découverte sur le chantier de la Bourse, d'un moule de lampe Atlante X (valve inférieure : fig. 62). Les lampes provenant des mêmes contextes et attribuables à cette production locale se différencient bien des modèles africains par une pâte chamois non engobée. Le deuxième exemple est celui de Générac, atelier qui a produit des vaisselles engobées et des lampes de type africain (fig. 56). Cinq moules en céramique y ont été découverts, attribuables au type Atlante VIII C et D du milieu du V ${ }^{\mathrm{e}}$. (Raynaud, 1982, fig. 5).

M. B. et C. R.

\section{LES CÉRAMIQUES COMMUNES}

Longtemps négligées par les études, les céramiques communes gallo-romaines ont néanmoins fait l'objet d'une attention particulière au cours des dernières décennies. Sans bénéficier encore d'une synthèse qui permettrait d'établir la localisation et la carte de la diffusion des nombreux ateliers, ces productions sont désormais assez connues pour que l'on commence à identifier des faciès régionaux ou interrégionaux. On n'oubliera pas cependant le caractère préliminaire d'une telle cartographie qui repose sur les seules études publiées et demeure un travail de seconde main. Une enquête approfondie qui retournerait aux documents 
eux-mêmes et intègrerait les nombreuses données inédites reste incontournable mais ne pourra être conduite sans la constitution d'une équipe réunissant l'ensemble des spécialistes. Les observations qui suivent entendent à la fois poser de premiers jalons et inviter à une entreprise ambitieuse dépassant le cadre du présent dossier.

Une dizaine de productions communes paraissent à même de rendre compte des principales tendances qui caractérisent la vaisselle de la fin de l'Antiquité. Arbitraire et partiel, ce choix s'est porté sur les céramiques les mieux identifiées et les mieux (ou les moins mal ?) datées. J'ai essayé autant que possible de couvrir l'ensemble des régions considérées, mais l'ampleur des lacunes reste considérable, particulièrement en ce qui concerne le Sud-Ouest où les références demeurent rares et n'autorisent encore aucune perception des aires de diffusion et des faciès.

Un constat général s'impose avant d'observer en détail les productions régionales, celui d'une continuité de la vaisselle commune au long de la période gallo-romaine. Tout en marquant des évolutions significatives, les techniques de fabrication, la morphologie et les usages des céramiques communes de la fin de l'Antiquité s'inscrivent dans le droit fil de celles du Haut-Empire, avec lesquelles nulle césure n'est discernable. Si de «nouvelles productions » et/ou de nouveaux ateliers apparaissent, cela se fait progressivement et non de manière synchrone. De même le répertoire des vases produits ne révèle aucune irruption de nouveaux modèles.

\section{LA GÉRAMIQUE COMMUNE GLAIRE}

Cruches, pichets, coupes et mortiers, entonnoirs et pots de fleurs aussi : une part essentielle de la poterie galloromaine provient d'ateliers utilisant une argile calcaire cuite en atmosphère oxydante, dénommée habituellement commune claire ou commune calcaire (DICOCER, 1993, p. 222-243). Issus de nombreuses officines œuvrant dans toutes les provinces de l'Empire occidental, ces produits font apparaître une typologie foisonnante, tandis que la chronologie n'apparaît pas encore clairement fixée, du fait de la longue durée de fabrication de certaines formes, cruches et mortiers notamment. Si la majeure partie de ces productions est dépourvue de revêtement, la production semi-fine, à engobe rouge ou orange, se perpétue jusqu'au $\mathrm{V}^{\mathrm{e}} \mathrm{s}$., notamment dans le Sud-Ouest où de nombreux fours sont connus, de Toulouse à Lectoure (Dieulafait et al., 1996). Si l'on ne peut encore caractériser un faciès tardo-antique au sein de cette production, il convient toutefois d'aborder la question en termes quantitatifs afin de rendre compte d'une permanence dans la composition du vaisselier.

Les données rassemblées dans le village gallo-romain de Lunel-Viel permettent de suivre l'évolution de la consommation du I ${ }^{\mathrm{er}} \mathrm{au} \mathrm{V} \mathrm{V}^{\mathrm{e}} \mathrm{s}$. Présente du $\mathrm{I}^{\mathrm{er}} \mathrm{au} \mathrm{IV}{ }^{\mathrm{e}} \mathrm{s}$. à hauteur de $30 \%$ à $40 \%$ des céramiques communes, la vaisselle à pâte claire connaît un déclin durant le $\mathrm{V}^{\mathrm{e}} \mathrm{s}$., chutant à $20 \%$ puis à $10 \%$ à la fin de ce siècle, avant de disparaître au début du VI ${ }^{\mathrm{e}}$ s. (Raynaud dir., 1990, p. 223). L'évolution est aussi sensible en ville, à Nîmes où la proportion se situe entre $15 \%$ et $18 \%$ à la fin du $\mathrm{IV}^{\mathrm{e}} \mathrm{s}$. et au début du $\mathrm{V}^{\mathrm{e}}$ s., puis entre $10 \%$ et $18 \%$ dans la première moitié du $\mathrm{V}^{\mathrm{e}} \mathrm{s}$. (Monteil dir., 1993, p. 169-185). La tendance est la même à Lyon où l'on note, entre le milieu du IV ${ }^{\mathrm{e}} \mathrm{s}$. et le milieu du siècle suivant, une baisse progressive de $27 \%$ à $10 \%$ (Ayala, 1998), avant que les niveaux des $\mathrm{VI}^{\mathrm{e}}$ et $\mathrm{VII}^{\mathrm{e}} \mathrm{s}$. ne voient disparaître cette poterie (Horry, 2000).

\section{LA CÉRAMIQUE NON TOURNÉE DU BAS-LANGUEDOC ET DU VAR}

Abandonnée dès la période augustéenne, la production de céramique non tournée réapparaît en Bas-Languedoc dans la seconde moitié du $\mathrm{III}^{\mathrm{e}} \mathrm{s}$. et se prolonge jusqu'au début du V $\mathrm{V}^{\mathrm{e}}$. (Raynaud dir., 1990, p. 231-235; DICOCER, 1993, p. 332). Aisément reconnaissable à sa pâte brun-gris, à fines particules de mica, à sa texture feuilletée et à son épiderme poli et satiné, cette production est régulièrement représentée, tant en contexte d'habitat que dans les nécropoles, dans un arc littoral entre le Rhône et l'Aude (Raynaud, in Solier dir., 1991, p. 228-232), peut-être même jusqu'au Tarn si l'on en juge par l'abondance de la vaisselle non tournée dans une nécropole de Castres, sans que l'on puisse encore affirmer qu'il s'agît d'une même production (Pons, 1998), le ou les atelier(s) de production n'étant pas connu(s). Plus à l'ouest, on ignore si cette production atteint la vallée de la Garonne.

Restreint à deux formes, assiette/plat et urne/gobelet et à quelques variantes, le répertoire typologique est bien représenté tant dans les habitats que dans les nécropoles, notamment à Lunel-Viel dont les tombes du Verdier ont livré 21 pièces servant de principales références typologiques (fig. 63).

La région varoise voit apparaître une production modelée $\mathrm{au} \mathrm{VI}^{\mathrm{e}}$ s., vaisselle caractérisée elle aussi par son répertoire essentiellement constitué d'urnes ovoïdes à bord mince éversé, ainsi que de quelques marmites hémisphériques et de couvercles coniques. La pâte granuleuse se caractérise 

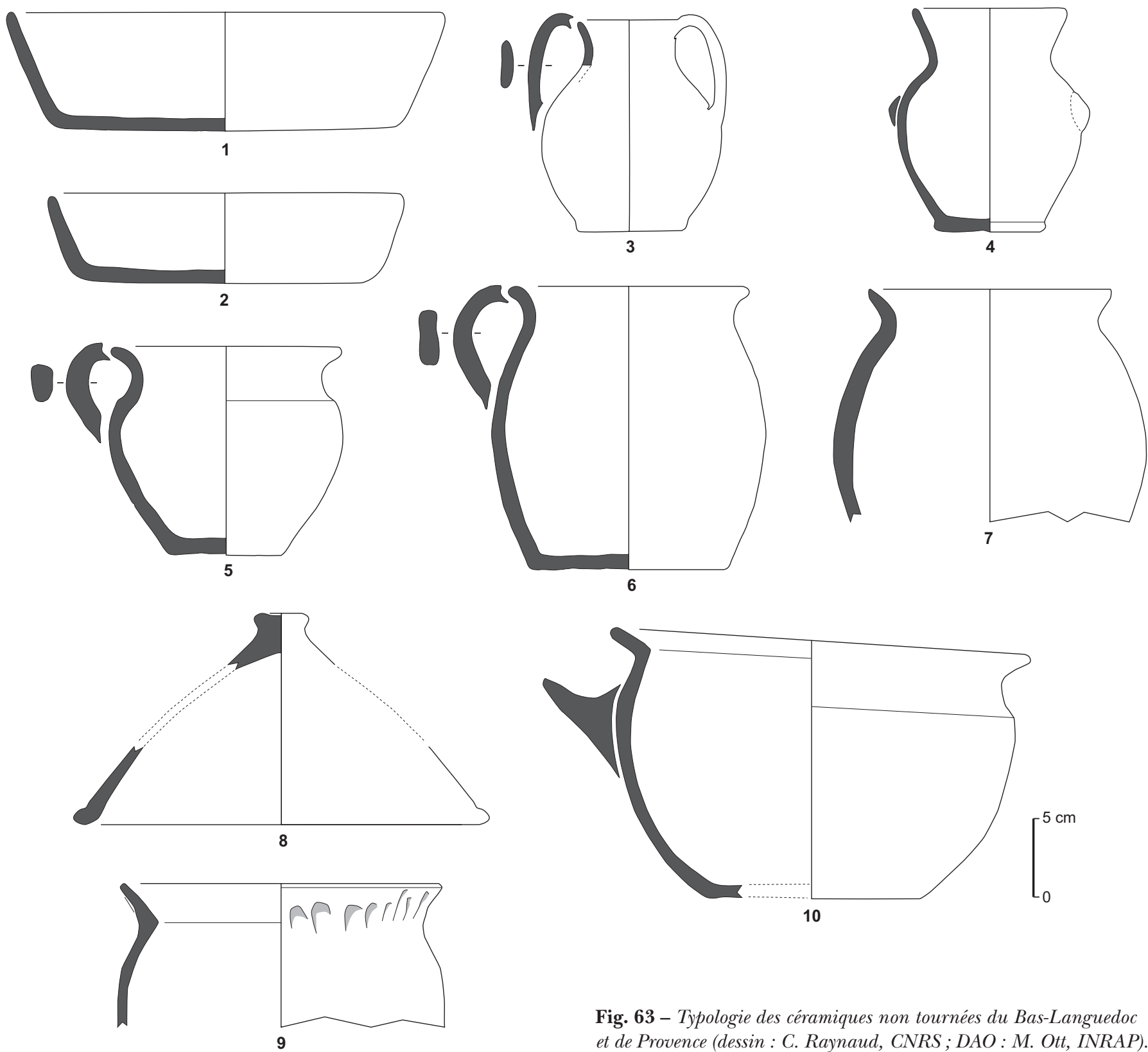

Fig. 63 - Typologie des céramiques non tournées du Bas-Languedoc et de Provence (dessin : C. Raynaud, CNRS ; DAO : M. Ott, INRAP).

par la présence de grains de quartz et de mica, ainsi que par sa couleur brun clair à brun foncé, avec souvent un pseudoengobe obtenu par polissage de l'épiderme. Les fonds sont souvent noircis, dénotant un usage culinaire. Cette vaisselle est régulièrement attestée tout au long des VI ${ }^{\mathrm{e}}$ et VII ${ }^{\mathrm{e}} \mathrm{s}$. sans connaître de diffusion hors de l'aire de production (Bérato, 1998).

Bien caractérisée par ses caractères techniques, la céramique non tournée du Bas-Languedoc et de BasseProvence ne constitue pas pour autant une singularité régionale : plusieurs régions méditerranéennes connaissent, $\mathrm{au} \mathrm{IV}^{\mathrm{e}} \mathrm{s}$. et/ou au $\mathrm{V}^{\mathrm{e}} \mathrm{s}$., un processus analogue : de l'Italie du Sud à la Taraconnaise, de nombreux ateliers diffusent largement des céramiques non tournées dont la typologie, dominée par des formes élémentaires, plats, terrines et marmites, évoque la commercialisation de produits alimentaires qui restent à identifier (Pasqualini, Tréglia, 2003). Loin de marquer un retour à une technique archaïsante, hypothèse insoutenable au moment où ces régions produisent les vaisselles fines les plus élaborées, luisante et 
estampée, le développement de la poterie modelée tardoantique pourrait au contraire dénoter une massification des échanges induisant la réalisation de produits de grande consommation, dont la qualité technique restait secondaire en regard de leur usage : conserver et transporter.

\section{LA CÉRAMIQUE À PISOLITHES DU BAS-LANGUEDOC ORIENTAL}

La poterie à pisolithes constitue l'un des principaux fossiles directeurs de la période tardo-antique en BasLanguedoc (DICOCER, 1993, p. 527-531). Cette poterie, caractérisée par une pâte à nodules ferrugineux, connaît les mêmes variantes de cuisson que les autres céramiques communes mais avec des couleurs généralement plus sombres: rose brique à brun foncé pour la postcuisson oxydante, gris à noir pour la post-cuisson réductrice. La surface est douce et résistante, fréquemment bosselée à cause des pisolithes et parfois craquelée à reflets bleutés, comme la poterie kaolinitique qu'il est parfois délicat de distinguer.

Tout autant que par ses caractères techniques, la céramique à pisolithes se singularise par des parois épaisses, par l'empâtement des profils ainsi que par la lourdeur des bords en bourrelets arrondis ou en amande. S'inscrivant dans la tradition antique, les fonds sont encore concaves et détachés de la panse par un bourrelet, mais celui-ci s'atrophie, marqué seulement par un ressaut. Bien marqués, les épaulements, carènes et gorges animent ces profils aux courbes régulières. La morphologie des vases renforce la singularité de cette production qui comporte un répertoire de vases culinaires : plats, marmites, urnes, mortiers, mais aussi un service de table: assiettes, bols et pichets. Un autre trait distinctif réside dans la prépondérance des formes ouvertes, plats et marmites, qui restent au contraire minoritaires parmi les céramiques communes sableuses ou kaolinitiques.

Apparue dans le dernier tiers $d u I^{e} s$. puis devenue majoritaire $\mathrm{au} \mathrm{V}^{\mathrm{e}} \mathrm{s}$., la céramique à pisolithes se situe à un tournant dans la production de vaisselle commune languedocienne. Elle marque en effet le début de la fabrication exclusive de poterie kaolinitique, trait majeur de l'artisanat potier du haut Moyen Âge. Dans un premier temps, la production est exclusivement à postcuisson oxydante jusque vers le milieu ou le dernier tiers $d u \mathrm{~V}^{\mathrm{e}} \mathrm{s}$., puis cette technique est concurrencée par la postcuisson réductrice qui, elle-même, devient exclusive vers le début $\mathrm{du}_{\mathrm{VI}}^{\mathrm{e}} \mathrm{s}$, , autre aspect du processus de «médiévalisation » de l'artisanat potier. Pour cette raison, la technique de cuisson constitue un indice chronologique significatif, le rapport quantitatif entre vases à postcuisson oxydante ou réductrice permettant d'étayer les observations typologiques. La fin de cette production reste mal cernée mais on peut l'envisager vers le milieu du $\mathrm{VI}^{\mathrm{e}}$ s., date à laquelle la poterie kaolinitique sans pisolithes devient prédominante. Après cette date, on ne connaît plus aucune production régionale utilisant l'argile à pisolithes (Raynaud, 2007).

Plusieurs ateliers ont été localisés au nord-ouest de Nîmes, où abondent les gisements d'argile kaolinitique à pisolithes. Des prospections méthodiques ont permis d'identifier deux ateliers de production caractérisés par d'abondants ratés de cuisson et des éléments de fours, en lisière au nord-est du Bois des Lens, sur les sites de la Devèze à Fons et de la Fontaine à Saint-Geniès-de-Malgoirès (CAG, 30/2, p. 361-362 et CAG, 30/3, p. 606). Deux ateliers sûrement identifiés, un troisième encore incertain, d'autres peut-être restant à localiser: la structure de production s'avère éclatée. Doit-on attribuer cette dispersion à un déterminisme géologique, les carrières d'argiles restant disséminées dans tout le massif, et/ou à une structure économique de petites entreprises peu ou pas connectées au niveau de leur mode d'exploitation? À ces questions, seules des fouilles offriraient des éléments de réponse. Voilà en tout cas évoquée la question de la production artisanale dans l'Antiquité tardive, question méconnue mais à laquelle les découvertes d'ateliers tardo-antiques, ceux de Générac, de la Ramière à Roquemaure et de la Quintarié à Clermontl'Hérault pour la vaisselle fine, les lampes ou la vaisselle commune, apportent un premier éclairage (CAG, 30/3, p. 541-542 ; Barberan, Pomarèdes, 1991 ; Raynaud, 1982). Hasard des découvertes ou reflet de la réalité, cet artisanat paraît, en l'état des données, essentiellement rural.

La céramique à pisolithes s'impose comme une production à large diffusion autour de l'épicentre nîmois où l'on note la plus forte densité de découvertes. Dans la région nîmoise où elles sont les mieux attestées, les céramiques à pisolithes connaissent une progression sensible au sein du vaisselier, en ville comme à la campagne. Dans les contextes du dernier tiers du IV ${ }^{\mathrm{e}}$ s., à Lunel-Viel ou Ambrussum, elles représentent $3 \%$ à $6 \%$ du total de la vaisselle (fine et commune confondues). Dans la première moitié $\mathrm{du} \mathrm{V}^{\mathrm{e}} \mathrm{s}$. leur part atteint plus de $6 \%$ à Lunel-Viel et de $9 \%$ à $16 \%$ à Nîmes, fouille des Halles (Monteil dir., 1993, p. 169-185). $\mathrm{Si}$, à Nîmes, la proportion reste stable jusqu'à la fin $\mathrm{du} \mathrm{V}^{\mathrm{e}} \mathrm{s}$., de $11 \%$ à $14 \%$, en revanche elle s'accroît à Lunel sur le site de Dassargues pour atteindre $40 \%$ (Garnier et al., 1995) 
et plus de $53 \%$ à Lunel-Viel. Au VI ${ }^{\mathrm{e}}$ s. enfin, le décompte est plus difficile en raison de l'étroite parenté avec la céramique kaolinitique, parfois difficile à distinguer : dans un premier temps j'avais classé en pisolithes (96\% de la vaisselle) l'ensemble des poteries de la rue de Sauve, à Nîmes, mais avec le recul j'en viens à penser qu'une part notable de cette série, peut-être $20 \%$, pourrait appartenir à la production kaolinitique (Raynaud dir., 1990 p. 245). Seules des analyses en laboratoire permettraient une évaluation plus ferme, mais en tout état de cause, l'hégémonie de la production à pisolithes ne fait aucun doute durant les premières décennies de ce siècle.

La fréquence décroît lorsque l'on s'éloigne du Languedoc oriental. Vers le sud, la diffusion couvre densément le littoral, de la Camargue jusqu'à la région d'Agde (Gomez et al., 2001). À l'ouest, la céramique à pisolithes est bien attestée dans l'arrière-pays de Montpellier, notamment dans la grotte de l'Hortus à Valflaunès (Démians d'Archimbaud, 1972) et au Roc de Pampelune à Argelliers (Schneider, 2003a et b), dans le bassin de Thau (Pellecuer, 1986, p. 106-112), puis dans la vallée de l'Hérault autour d'Aniane en particulier (Schneider, Paya, 1995), ou près de Pézenas (Mauné, 1997-1998, p. 14). Plus à l'ouest, la région de Béziers ne livre aucune donnée mais ce silence n'est probablement pas significatif dans la mesure où notre vaisselle est présente à Narbonne, point le plus occidental recensé à ce jour (Raynaud, 1991a et b). Plus à l'ouest, la céramique à pisolithes n'est attestée ni en Lauragais ni dans les Corbières où les faciès céramologiques sont bien cernés (Térès, Rigoir, 2001). Pourtant, « quelques fragments de céramique à pisolithes » sont mentionnés à Toulouse dans un contexte de la fin $d u I^{e}$ ou du V $V^{e}$ s., sans que l'on soit assuré qu'il s'agît bien de la production gardoise (Catalo et al., 1998, p. 7).

Vers l'est, la production à pisolithes franchit le Rhône pour atteindre Arles où elle est aussi bien représentée qu'à Nîmes. La représentation faiblit à Eyguières (Pelletier et al., 2000, p. 308), à Saint-Blaise (Démians d'Archimbaud dir., 1994, p. 187) et à Marseille où l'on ne signale plus que quelques fragments (CATHMA, 1986, fig. 14). L'enquête est plus difficile vers le nord où les céramiques à pisolithes ne sont réellement identifiées que dans la région de Bagnolssur-Cèze, sur les oppida de Saint-Vincent (Gaujac), du Camp de César (Laudun) et à Lombren (Vénéjan) (CATHMA, 1993, p. 116-117). Plus au nord encore, la grotte du Pontiar, à Vallon-Pont-d'Arc, a livré une céramique kaolinitique d'un faciès très proche de la vaisselle à pisolithes mais un examen visuel de ces pièces serait nécessaire pour en vérifier l'attribution (Alcamo, Lagrand, 1985, p. 205). Toujours dans l'Ardèche, les fouilles de la cité d'Alba auraient livré des céramiques à pisolithes. Sur la rive gauche du Rhône, la production gardoise est bien attestée dans la région d'Avignon, notamment dans des nécropoles des $\mathrm{IV}^{\mathrm{e}}$ et $\mathrm{V}^{\mathrm{e}} \mathrm{s}$. (Mouraret, 2002). Vers le nord, la diffusion ne semble pas atteindre le Tricastin où mes recherches ont révélé un faciès très proche par son répertoire, mais dans une argile kaolinitique sans pisolithes (Odiot dir., 1992, p. 124-131).

Ollae, urnes, pichets, marmites, bols, plats et mortiers : par la diversité de la vaisselle autant que par le choix des formes, la production à pisolithes prolonge la tradition de la poterie commune gallo-romaine (fig. 64). Cette vaisselle retient cependant l'attention par les singularités évoquées précédemment: lourdeur des profils et empâtement des bords. Ces détails en viendraient même à masquer l'enracinement du répertoire si l'on ne soulignait les nombreux «héritages» perpétuant les productions antérieures. Ces emprunts s'avèrent assez nombreux pour asseoir l'idée d'une stabilité des cadres de production potière et du contexte culturel. Une continuité d'autant plus nette que le répertoire des formes ne révèle aucun emprunt à d'autres poteries que commune et régionale : malgré leur association récurrente dans les contextes de consommation, les poteries à pisolithes n'empruntent aucune forme ni aucun trait aux productions fines, africaines, rhodaniennes (Luisante) ou régionales (estampée DS.P.). Apparaissent, en revanche, de nombreuses parentés avec la vaisselle commune des III $^{\mathrm{e}}$ et $\mathrm{IV}^{\mathrm{e}} \mathrm{s}$.

Lorsque s'efface la céramique à pisolithes, peut-être au milieu du $\mathrm{VI}^{\mathrm{e}}$ s. ou un peu plus tard, on peut donc dire que l'essentiel de la vaisselle commune de la Gaule Narbonnaise s'inscrit encore pleinement dans une ambiance antiquisante. Le premier élément d'une «médiévalisation" de l'équipement culinaire et de table est cependant acquis: désormais la vaisselle est uniformément grise.

\section{LA CÉRAMIQUE À GRAINS DE QUARTZ, DE L'HÉRAULT À LA GARONNE ET EN AVEYRON}

La vaisselle commune de la fin du $\operatorname{III}^{\mathrm{e}} \mathrm{s}$. et $\mathrm{du} \mathrm{IV}^{\mathrm{e}} \mathrm{s}$. apparaît dominée par une gamme de productions siliceuses à parois lissées ou lustrées, attestées de Narbonne (Solier dir., 1991, p. 221-225) à Rodez (Boudartchouk, Llech, 1993, p. 156), avec un large répertoire directement issu des productions du Haut-Empire : mortiers à collerette, bols hémisphériques, urnes ovoïdes à bord en bourrelet quadrangulaire (id., ibid., fig. 2 et 3). Les premiers jalons ne permettent pas encore de cerner la ou les aires de produc- 

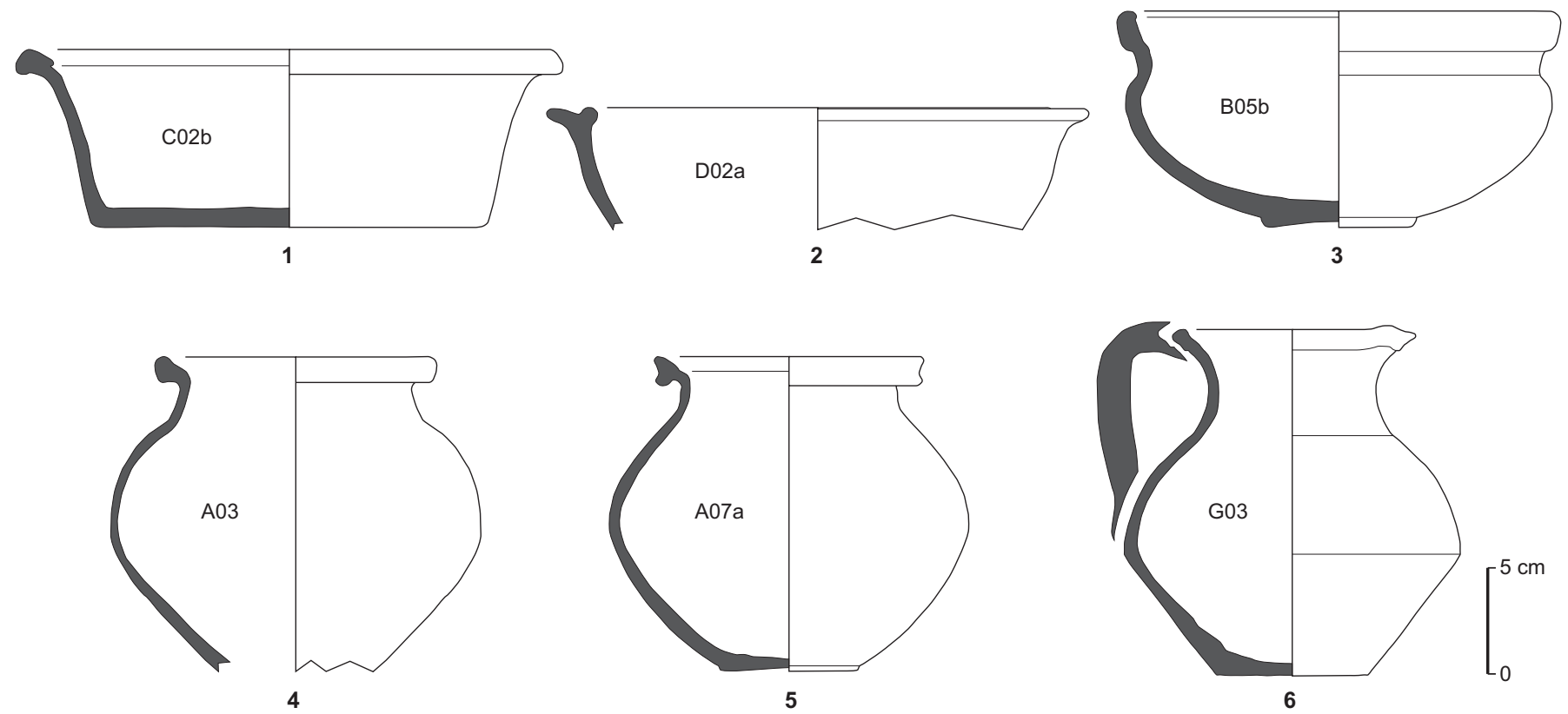

Fig. 64 - Abrégé typologique des céramiques à pisolithes du Bas-Languedoc oriental (dessin : C. Raynaud, CNRS; DAO : M. Ott, INRAP).

tion et de distribution de ce « faciès » aux contours encore incertains, dont la caractérisation nécessitera une étude approfondie.

À partir de la fin du $\mathrm{IV}^{\mathrm{e}} \mathrm{s}$. et aux deux siècles suivants, le Bas-Languedoc occidental et la région toulousaine, ainsi que la région des Causses, voient se diffuser une poterie grise à grains de quartz et de calcite, production montée au tour rapide et comportant de nombreuses irrégularités, que l'on nomme tantôt céramique de «Maubert», tantôt céramique de «Pabiran", du nom des établissements où cette céramique fut pour la première fois identifiée (Mauné, 1997-1998 ; Boudartchouk, Llech, 1993, p. 159160). Le répertoire des formes marque une première étape vers la simplification du haut Moyen Âge, avec trois types seulement, analogues aux céramiques à pisolithes : le pot ou urne globulaire à bord en poulie, qui représente près de $80 \%$ des pièces, le plat tronconique, le mortier à collerette (fig. 65). Une quatrième forme, le bol caréné à lèvre aplatie, trouve au contraire des parallèles dans d'autres productions, d'une part la céramique calcaire engobée du $\mathrm{IV}^{\mathrm{e}} \mathrm{s}$. (DICOCER, 1993, p. 200, type B6), d'autre part la céramique bistre du Val de Saône des $\mathrm{V}^{\mathrm{e}}-\mathrm{VI}^{\mathrm{e}} \mathrm{s}$. (Faure-Boucharlat dir., 2001, p. 67 et p. 227-233).

$\mathrm{Au} \mathrm{V}{ }^{\mathrm{e}}$ s. et $\mathrm{au} \mathrm{VI}{ }^{\mathrm{e}} \mathrm{s}$., cette production granuleuse rayonne dans une vaste région s'étendant jusqu'aux Corbières et le Roussillon au sud-ouest, depuis un épicentre dans la basse vallée de l'Hérault, sans qu'aucun atelier n'y soit encore localisé. La diffusion vers le nord atteindrait la région des Causses où elle est connue sous la dénomination de céramique de Maubert. Vers l'est, la diffusion semble faiblir dans la région de Loupian où elle est peu attestée, mais on la retrouve jusqu'à Maguelone. Plus à l'est encore, elle reste absente à Lunel-Viel et dans la région nîmoise.

Une production grise siliceuse est attestée à Toulouse au $\mathrm{V}^{\mathrm{e}} \mathrm{s}$. avec un répertoire limité à trois formes, l'urne ovoïde à bord en poulie, le bol hémisphérique à lèvre épaissie et le mortier à collerette (Catalo et al., 1998, p. 6-7). «Proches d'exemplaires mis au jour à Rodez », ces poteries esquissent un faciès toulousain qui pourrait s'apparenter à la production de Maubert, mais les documents comparatifs font encore défaut.

Première confrontation, premières constatations : l'aire de diffusion des céramiques «à grains blancs" ne représente pas une entité homogène, les aires de répartition ne sont pas exclusives, mais au contraire se chevauchent, la diffusion de la céramique à pisolithes recouvre la moitié orientale de l'aire «à grains de quartz», et réciproquement. D'autre part il n'est pas inutile de noter, contre l'idée d'une économie imbriquée dans le politique, la totale indépendance de la diffusion des poteries à l'égard des cités gallo-romaines : en aucun cas on ne peut parler d'une production nîmoise à propos des céramiques à pisolithes, pas plus que l'on ne peut attribuer la poterie «à grains blancs » à une cité particulière. Dernière observation, la diffusion 

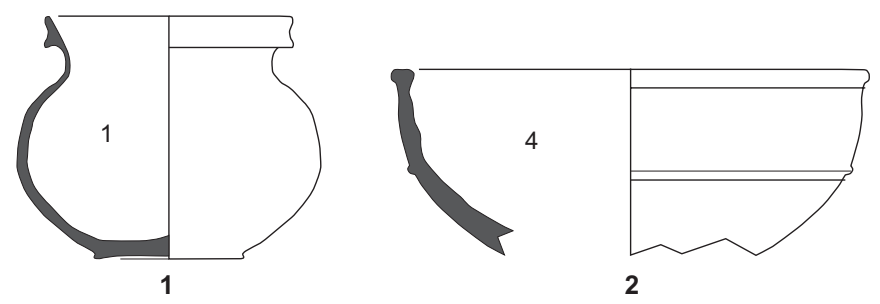

2
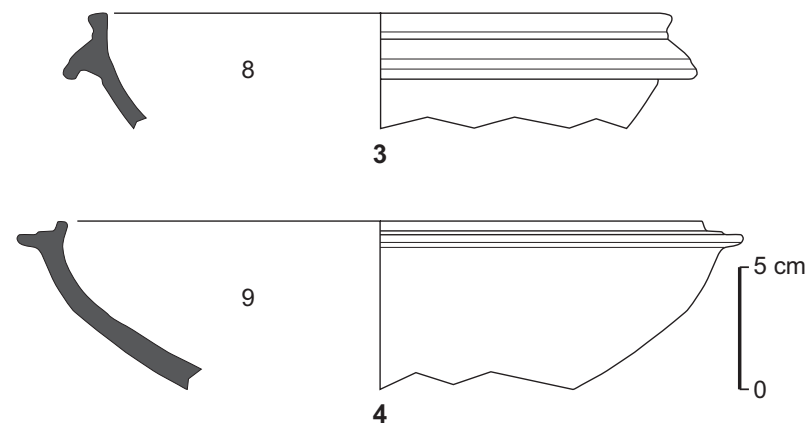

Fig. 65 - Typologie des céramiques "à grains blancs » du Languedoc occidental et des Causses (d'après Mauné, 1997-1998; DAO : M. Ott, INRAP).

des céramiques communes est tout sauf «locale» dans la mesure où la diffusion s'étend dans un rayon de $50 \mathrm{~km}$ à $150 \mathrm{~km}$, voire plus encore.

En l'état des connaissances, la diffusion des productions à pisolithes et à grains blancs semble assurer l'essentiel de l'approvisionnement en céramique commune de la Septimanie wisigothique. Enchâssés dans ces aires de diffusion, d'autres ateliers semblent répondre à une consommation plus locale, à la Quintarié (Clermont-l'Hérault) et à la Ramière (Roquemaure) (fig. 66), à moins que la méconnaissance de leur diffusion ne fonde seule cette première hypothèse. Si, à la Ramière, la production kaolinitique s'inspire surtout d'un répertoire de vaisselle de table, avec bols et assiettes, la production commune de la Quintarié - qui produit aussi de la vaisselle fine et semi-fine - révèle un répertoire très proche des vaisselles à pisolithes et de «Pabiran ». Faut-il encore envisager d'autres productions? Le cas est avéré en Languedoc oriental où les pisolithes subissent dès le $\mathrm{V}^{\mathrm{e}} \mathrm{s}$. la concurrence, d'abord discrète, puis envahissante au $\mathrm{VI}^{\mathrm{e}} \mathrm{s}$., de la production kaolinitique rhodanienne. D'autres ateliers locaux restent certainement à découvrir dans des secteurs délaissés par la recherche, mais il paraît acquis que, pour l'essentiel, l'approvisionnement régional reposait sur les deux principales productions, les seules que l'on reconnaît partout où des fouilles étendues ont livré des lots de mobilier conséquents.

C. R.

\section{LES CÉRAMIQUES COMMUNES GRISES DE L'ANTIQUITÉ TARDIVE EN PROVENGE}

Cette catégorie de céramique se caractérise par sa pâte réfractaire assez grossière dont la couleur, variant du gris clair au gris foncé selon les objets, est due à la cuisson en atmosphère réductrice. L'aire de diffusion de cette technique recouvre, en Provence, les départements des Bouchesdu-Rhône, des Alpes-de-Haute-Provence et du Vaucluse (Pelletier, 1997, p. 112) ; sa limite orientale est située dans le centre du Var et au nord d'une large frange côtière à l'est de Toulon où les productions cuites en atmosphère oxydante sont pratiquement exclusives. Comme à l'ouest du Rhône, la cuisson en atmosphère réductrice apparaît en Provence dans le courant de la seconde moitié $d u \mathrm{~V}^{\mathrm{e}} \mathrm{s}$. et sa production se poursuit tout au long du VII ${ }^{\mathrm{e}} \mathrm{s}$. Durant cette longue période, le répertoire des formes ne change pratiquement pas, comme les habitudes culinaires qu'il reflète, mais on observe néanmoins une certaine évolution notamment avec le remplacement progressif des différentes vaisselles (Claires D et Luisantes importées ou DS.P. régionales en pâte calcaire) par des objets comparables en pâte commune grise. De même, la typologie des rebords présente des modifications dont les détails permettent de préciser la chronologie. Il faut aussi remarquer que les vaisselles importées, toujours bien présentes sur les côtes, ne pénètrent qu'en petite quantité à l'intérieur des terres à partir $\mathrm{du} \mathrm{VI}^{\mathrm{e}} \mathrm{s}$.

Si aucun four n'a encore été mis au jour, l'étude des matériels fait penser à des lieux de fabrication multiples dont le rayon de commercialisation devait être limité à quelques dizaines de kilomètres. Les principaux centres potiers se trouvaient d'une part dans la région marseillaise, où les analyses géochimiques situent dans l'arrière-pays les gisements d'argile qui ont alimenté les ateliers fournissant la ville et ses environs (Pelletier et al., 1995 ; Pelletier, Rigoir, 1998), et d'autre part dans la vallée du Rhône et la région d'Apt.

\section{LES FORMES LES PLUS COURANTES}

Les proportions des différentes formes observées sur deux ensembles de référence (Saint-Blaise et Gardanne) comprenant chacun plus de 400 objets ont montré que les poteries les plus utilisées, les ollae (forme A), marmites (MA), coupes et coupelles (B), ainsi que les couvercles (E) auxquels elles doivent être associées représentent environ $80 \%$ du répertoire des communes grises. Les autres formes, totalisant $20 \%$ des individus, ont été qualifiées de « rares ». 

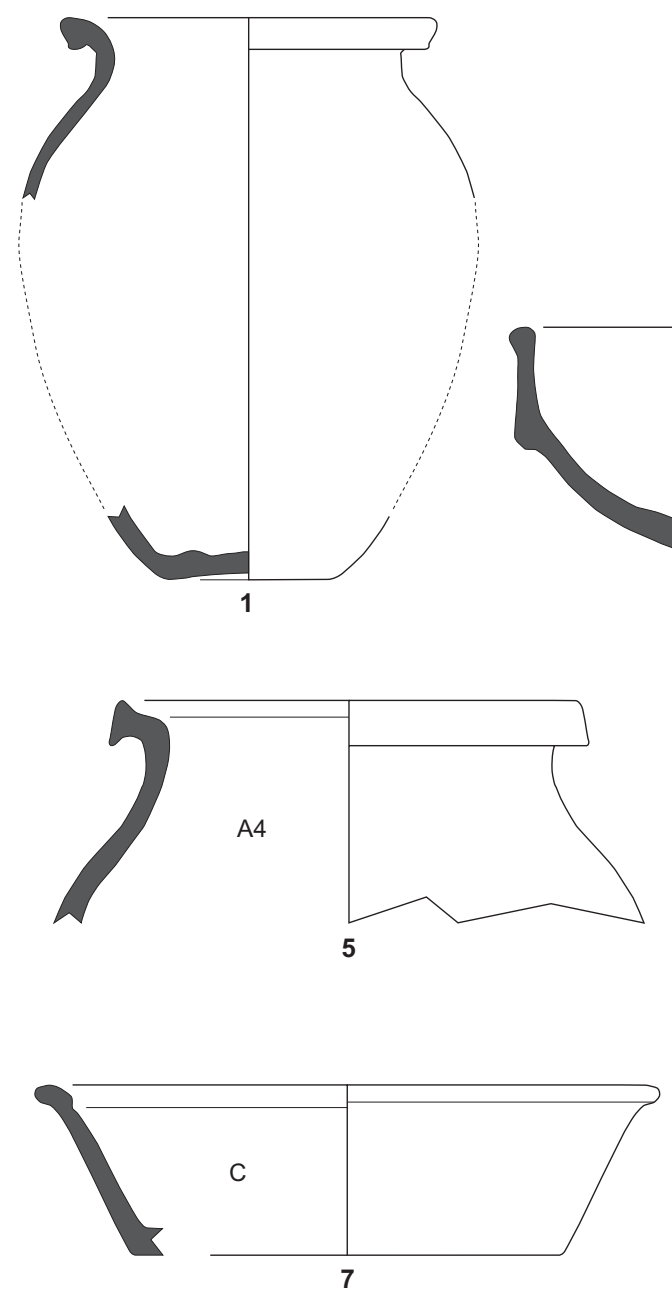
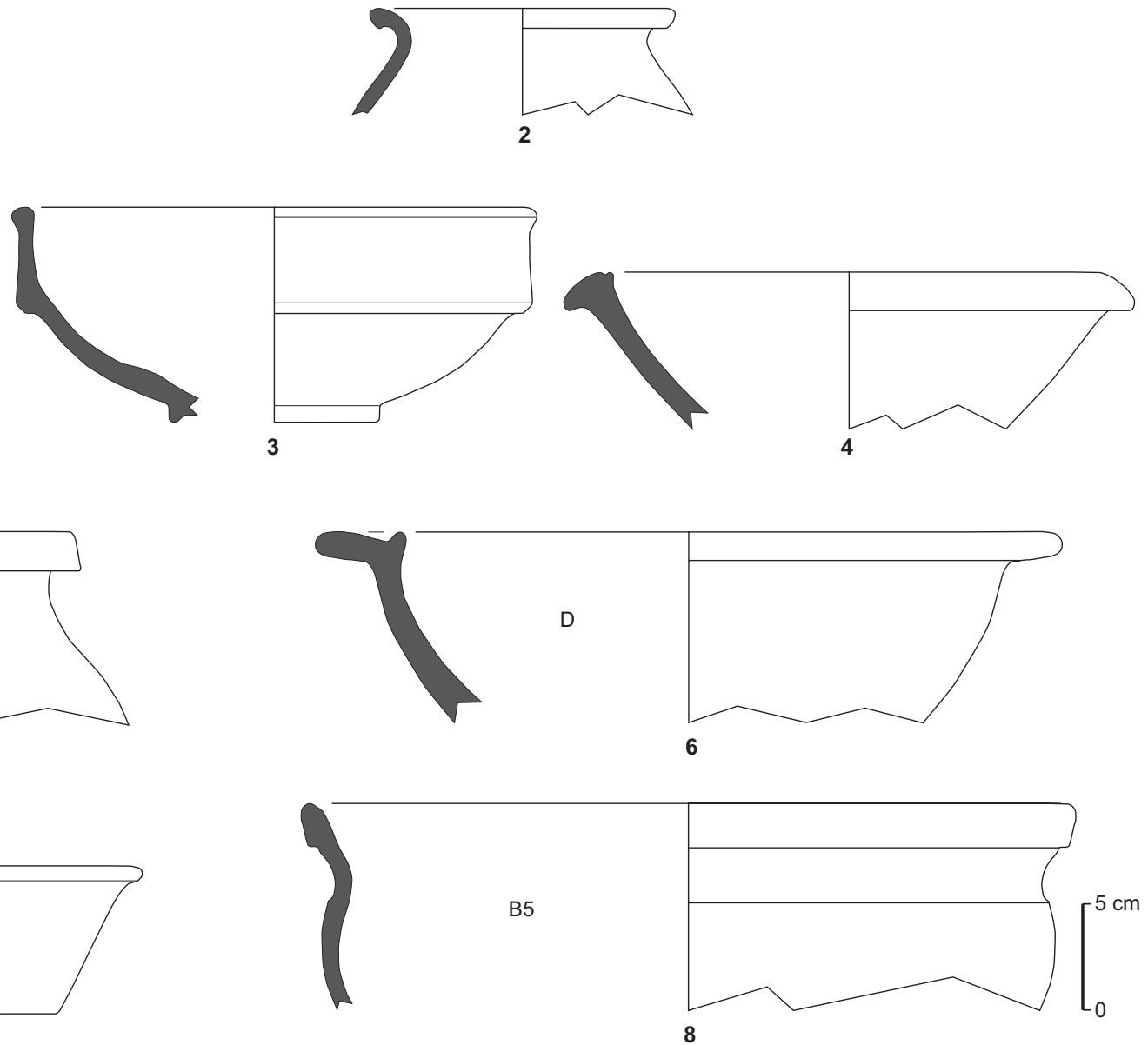

Fig. 66 - Typologie des céramiques des ateliers de Roquemaure, la Ramière (1-4) et de Clermont-l'Hérault, la Quintarié (5-8) (d'après CAG, 30/3 ; DAO : M. Ott, INRAP).

\section{Les formes fermées : ollae et marmites}

Ces poteries, dont le total équivaut à la moitié des objets recensés, étaient destinées pour l'essentiel à un usage culinaire, mais aussi au service de la table (fig. 67). Le catalogue est largement dominé par les ollae (formes A), pots à tout faire d'une contenance moyenne de 1,5 à 3 litres, dont le profil un peu plus large que haut, avec diamètre maximum placé un peu au-dessus de la mi-hauteur, reprend les modèles des urnes antiques. Leurs fonds, qui comportent parfois l'ébauche d'un petit pied, sont plus ou moins plats et toujours étroits, moins de la moitié du diamètre maximum ; le diamètre du rebord équivaut en moyenne aux deux tiers du diamètre maximum (Pelletier, 1998, p. 218-219). Les formes A sont dépourvues d'anses. Beaucoup moins fré- quentes, les marmites (formes MA) sont d'allure tout à fait comparable mais comportent deux anses diamétralement opposées, fixées sur le rebord ; leur contenance est de 3 à 5 litres. Sur ces deux types d'objets peuvent s'adapter des couvercles au profil tronconique munis d'un bouton de préhension (forme E).

\section{Typologie et datation des rebords}

On ne retrouve à Marseille qu'une partie des rebords de la typologie établie à partir de séries découvertes sur différents sites provençaux (Pelletier, Vallauri, 1994; Pelletier, 1997). Les types les plus courants sont les formes A1 (profil simple, avec ou sans bourrelet), A2 (profil simple à gorge interne plus ou moins marquée) et A3 (fig. 67 et 68). 

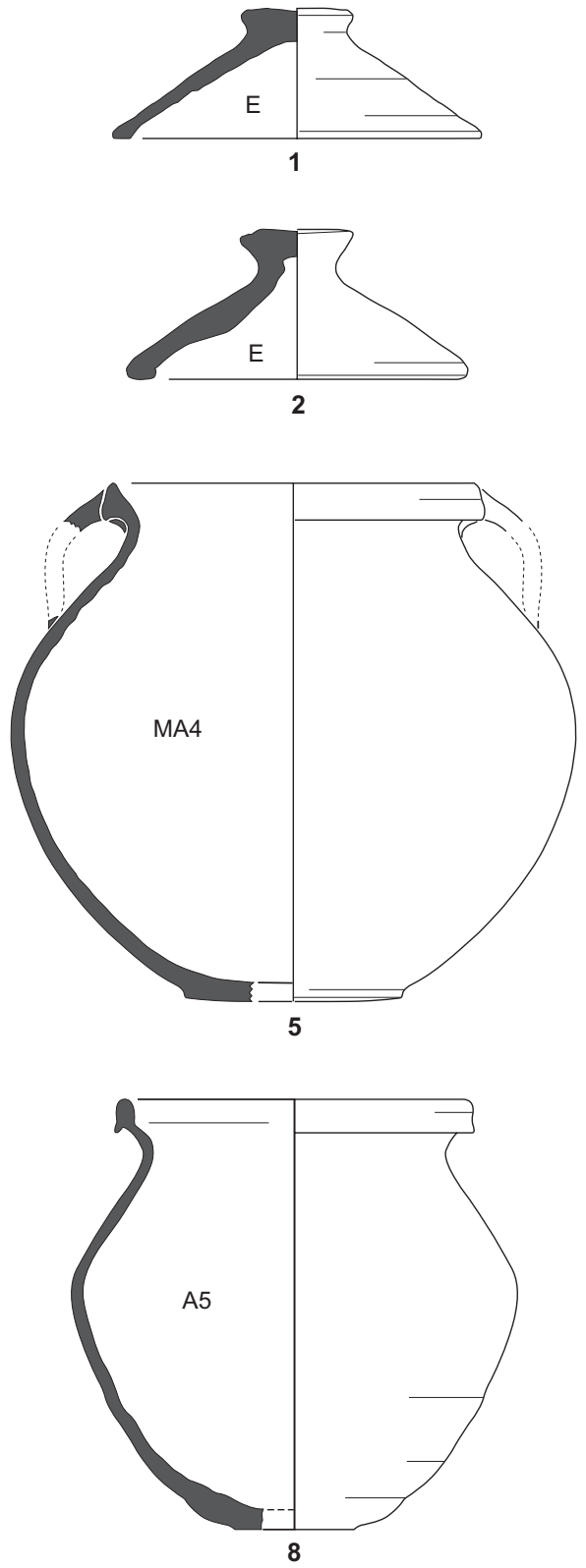
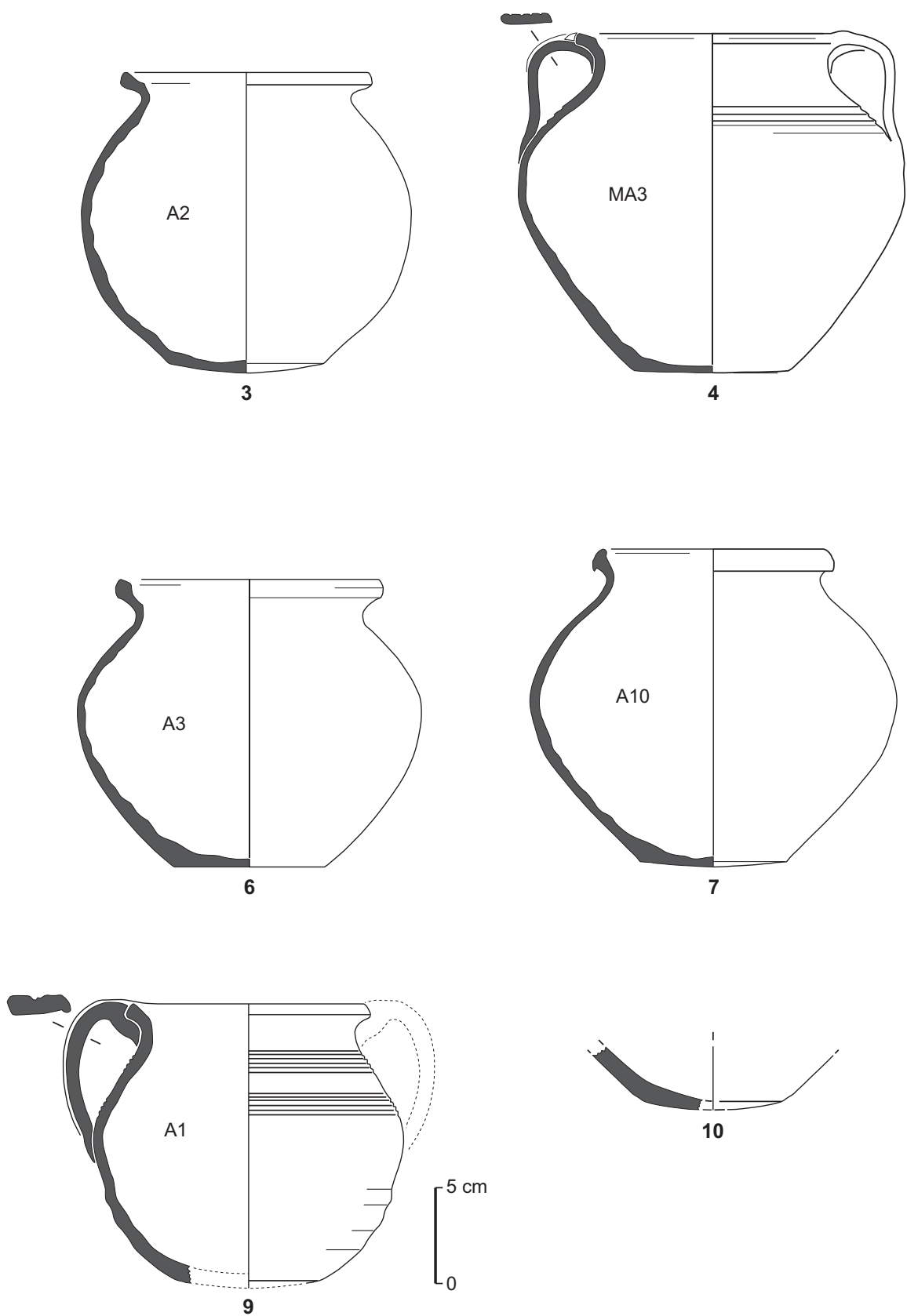

Fig. 67 - Typologie des formes des céramiques à pâte grise provençales : A (ollae), MA (marmites) et E (couvercles). Origine des exemples présentés : 1, Marseille, la Bourse ; 2, Velaux ; 3, 5, Gardanne ; 4, 6, Marseille, Bon-Jésus ; 7, Marseille, la Fourbine ; 8 (avec fond de transition), Marseille, Alcazar; 9 (avec fond de transition), Eyguières ; 10 (avec fond de transition), Jouques (DAO : J.-P. Pelletier, CNRS).

Ils constituent la majorité des éléments dès la fin du $\mathrm{V}^{\mathrm{e}} \mathrm{s}$. et se retrouvent de façon continue jusqu'au VII ${ }^{\mathrm{e}} \mathrm{s}$. (Bonifay, Pelletier, 1983 et 1998 ; Pelletier, 1998 ; Bien, 2001) ; les profils A3 de section plus ou moins carrée présentent des variantes de détail, et la forme triangulaire A3T est bien représentée à Gardanne. Les rebords A4 (section trian- gulaire), très répandus à partir $\mathrm{du} \mathrm{VI}^{\mathrm{e}} \mathrm{s}$. dans la vallée du Rhône et le Languedoc, sont assez rarement attestés à Marseille mais bien présents dans ses environs. Peu fréquente, la forme A5, identifiée à Saint-Blaise, comporte toujours une gorge en poulie ou réa. Très caractéristique, la forme A6 (section en bandeau à bourrelet interne) 


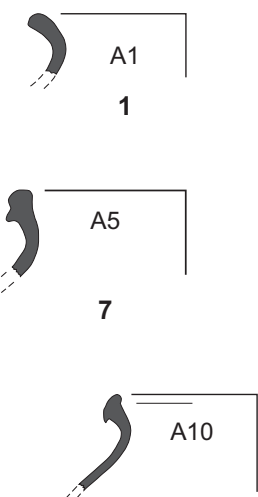

13

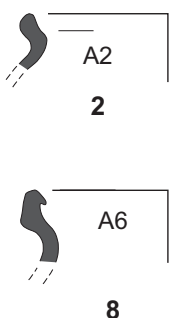

8

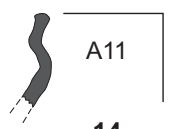

14
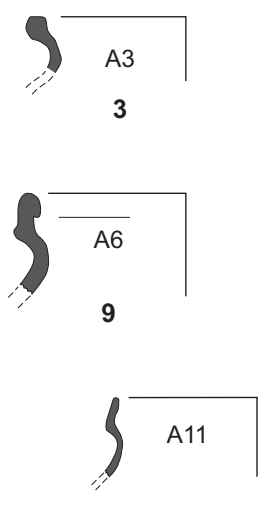

15

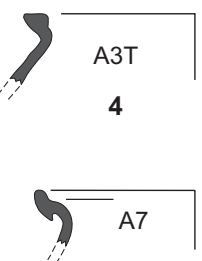

10

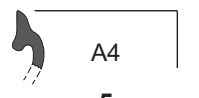

5

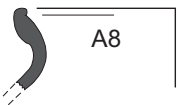

11

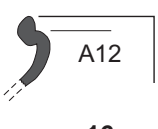

16

17

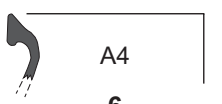

6

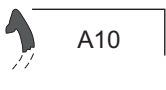

12

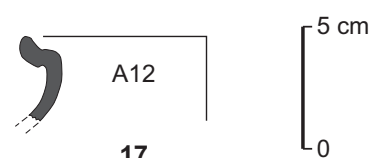

Fig. 68 - Typologie des bords des formes des céramiques à pâte grise provençales : A (ollae) et MA (marmites). Origine des exemples présentés : 1, Marseille, la Bourse ; 2, 3, 8, Marseille, Saint-Victor ; 4, Gardanne ; 5, 13, Marseille, la Fourbine ; 6, Lancon-de-Provence, oppidum de Constantine ; 7, 11, Saint-Blaise ; 9, Eyguières ; 10, 15, étang de Berre, Saint-Estève-de-Ménerbes ; 12, Marseille, Bon-Jésus ; 14, Marseille, Providence; 16, 17, Apt (DAO : J.-P. Pelletier, CNRS).

n'apparaît que dans la seconde moitié ou à la fin du $\mathrm{VI}^{\mathrm{e}} \mathrm{s}$. et reste fréquente au VII ${ }^{\mathrm{e}} \mathrm{s}$. La forme A7 qui apparaît dans la première moitié $\mathrm{du} \mathrm{VI}{ }^{\mathrm{e}} \mathrm{s}$. est surtout présente à l'ouest de la Provence et semble avoir été fabriquée dans la vallée du Rhône. La forme A8, dont la section est comparable à celle des rebords de forme $\mathrm{B} 5$ produits dans la vallée du Rhône et en Languedoc, figure dans des contextes de la fin du $\mathrm{V}^{\mathrm{e}}$ s. et $\mathrm{du} \mathrm{VI}^{\mathrm{e}}$ s., et reste assez rare. La forme A10 (section triangulaire, avec une partie plus ou moins fine pendante à l'extérieur), assez exceptionnelle, ne se retrouve que dans des niveaux du VII ${ }^{\mathrm{e}} \mathrm{s}$. La forme A11 (section en bandeau mince déporté au-dessus de l'épaulement très fin), retrouvée en plusieurs exemplaires à Saint-Victor (Marseille), est attestée au VII ${ }^{\mathrm{e}} \mathrm{s}$. dans divers contextes marseillais et provençaux. Les formes A12, fabriquées dans la région d'Apt comme les formes ouvertes B9, G et $\mathrm{H}$, ont connu à la fin $\mathrm{du} \mathrm{V}^{\mathrm{e}} \mathrm{s}$. et au $\mathrm{VI}^{\mathrm{e}} \mathrm{s}$. une faible diffusion en Provence occidentale, jusque dans la région aixoise où les productions marseillaises sont largement majoritaires.

Les fonds des formes A, comme ceux des formes B, restent toujours assez étroits et à peu près plats jusqu'au VII ${ }^{\mathrm{e}}$ s., mais on observe parfois dans les rares niveaux de cette séquence (Pelletier, 1997, p. 115-116; Pelletier et al., 2000 ; Bien, 2001, fig. 524, no 6) l'apparition sur des formes A de fonds de transition (fig. 67, nos 8 à 10). Avec une certaine épaisseur des parois en bas de panse qui constitue encore un caractère antique, ils présentent une convexité marquée tendant au bombement qui semble marquer le passage entre la fin de l'Antiquité tardive et le haut Moyen Âge, caractérisé par la présence des larges fonds bombés aux parois assez minces.

\section{Les formes ouvertes : coupes et coupelles}

Les coupes et coupelles (formes B), qui représentent le quart du total des objets, comportent des fonds identiques à ceux des formes $\mathrm{A}$ et $\mathrm{MA}$, mais avec un angle plus réduit entre le bas de la panse et l'horizontale (fig. 69). Comme les formes A, elles montrent des proportions constantes : le diamètre maximum des panses, soit plus du double de la hauteur, est à peine inférieur à celui des rebords évasés. Leurs dimensions peuvent varier du simple au double.

\section{Typologie et datation des rebords}

En ce qui concerne les formes les plus répandues dans toute la Provence, les types B1, B2 et B3 reprennent les caractères des ollae $\mathrm{A} 1, \mathrm{~A} 2$ et A3; ils apparaissent dans la seconde moitié $\mathrm{du} \mathrm{V}^{\mathrm{e}} \mathrm{s}$. et restent très courants au $\mathrm{VI}^{\mathrm{e}} \mathrm{s}$. La forme B3B constitue avec son bourrelet interne une variante bien attestée à Gardanne au $\mathrm{VI}^{\mathrm{e}} \mathrm{s}$, mais aussi à Saint-Blaise : son profil est comparable à des productions ligures en pâte rouge. La forme B4, avec une section en bandeau plus ou moins allongé, est assez rare. La forme B5, de même section que les ollae A8 originaires de la vallée du Rhône, se retrouve aussi en Provence occidentale dans des contextes de la fin $d u \mathrm{~V}^{\mathrm{e}} \mathrm{s}$. et du VI ${ }^{\mathrm{e}} \mathrm{s}$. La forme B6, identifiée à Saint-Blaise et mieux connue dans la vallée du Rhône $\mathrm{au} \mathrm{VI}^{\mathrm{e}}$ s., reste assez exceptionnelle. La forme B7, bien attestée à Gardanne, se définit surtout par une panse assez rectiligne, sans épaulement, et un diamètre supérieur à la moyenne avec des rebords de différents types. Assez rare, la forme $\mathrm{B} 8$, avec une section en bandeau mince, présente 


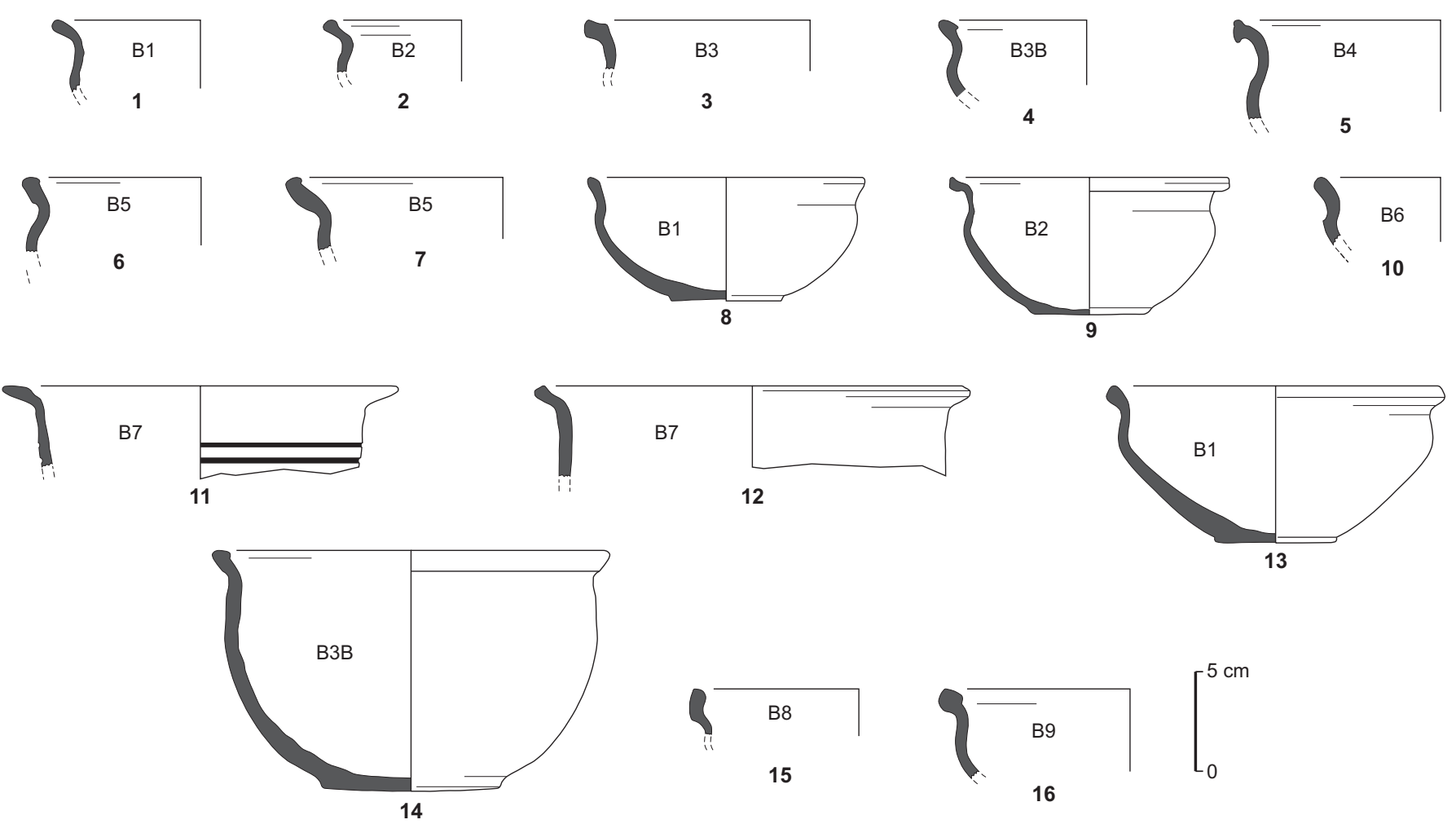

Fig. 69 - Typologie des rebords des formes $B$ des céramiques à pâte grise provençales. Origine des exemples présentés : 1, 2, 3, 9, Marseille, Saint-Victor ; 4, 6, 11, 12, 14, 15, Gardanne ; 5, 7, 8, 10, 13, Saint-Blaise ; 16, Apt (DAO : J.-P. Pelletier, CNRS).

des analogies avec la forme d'olla A11, mais apparaît dans des contextes un peu moins tardifs, du courant du VI ${ }^{\mathrm{e}} \mathrm{s}$. La forme B9 est propre aux ateliers de la région d'Apt à la fin $\mathrm{du} \mathrm{V} \mathrm{V}^{\mathrm{e}} \mathrm{s}$. et au VI ${ }^{\mathrm{e}} \mathrm{s}$. À ce jour, les formes ouvertes connues dans les niveaux du VII ${ }^{\mathrm{e}}$ s. restent peu nombreuses.

\section{LES FORMES RARES}

Les mortiers (forme $\mathrm{D}$, fig. 70, $\mathrm{n}^{\mathrm{O}} 1$ ), qui concurrencent ou tendent à remplacer les objets issus d'autres productions régionales ou importées, apparaissent au $\mathrm{VI}^{\mathrm{e}} \mathrm{s}$. et représentent encore une quantité non négligeable du matériel (environ $5 \%$ du total des objets) jusqu'au siècle suivant.

Les autres formes de poteries « rares ", assez exceptionnelles, ne représentent que $10 \%$ environ du total des objets. On ne connaît pas d'amphorettes complètes (forme F, dont l'équivalent existe en DS.P., forme 63), mais les rebords en poulie de ces productions $\mathrm{du} \mathrm{VI}^{\mathrm{e}} \mathrm{s}$. sont bien typiques (fig. 71, $\mathrm{n}^{\text {os }} 7$ et 8). Les cruches (forme K, fig. 71, $\mathrm{n}^{\text {os }} 9$ et 10) existent dans diverses productions et naturellement elles ont été aussi fabriquées en commune grise dès la fin du $\mathrm{V}^{\mathrm{e}} \mathrm{s}$., comme les gobelets (forme L1, fig. 70, $\mathrm{n}^{\mathrm{o}} 4$ ) et les petits bols (forme L2, fig. 70, $\mathrm{n}^{\mathrm{O}} 3$ ) dont la panse présente souvent un décor d'incisions horizontales; un bol présente un profil proche des couvercles E (forme L/E, fig. 70, no 5 ). Aux formes $\mathrm{F}$ et $\mathrm{K}$, dont le rebord est plus ou moins étroit, on peut associer des éléments retrouvés en exemplaires uniques qui semblent destinés à leur servir de bouchon (fig. 71, $n^{0} 1$ ) ou de couvercle à collerette (fig. 71, $n^{0} 3$ ) ; un autre couvercle à collerette, pouvant aussi servir de bol (fig. 71, $\mathrm{n}^{\mathrm{o}} 2$ ), est sans doute une copie de la forme DS.P. 54.

Typiques des productions marseillaises, les formes $\mathrm{O}$, marmites à large ouverture et tenons de préhension (fig. 71, $\mathrm{n}^{\mathrm{o}} 4$ ), se retrouvent dans des contextes de la fin du $\mathrm{VI}^{\mathrm{e}} \mathrm{s}$. et du VII ${ }^{\mathrm{e}}$ s. (Pelletier, Vallauri, 1994, p. 179-180 ; Bien, 1998, p. 281 ; Bonifay, Pelletier, 1998, p. 371). La forme AB 36 à bec tubulaire (fig. 71, no 5), connue par deux exemplaires à Glanum et à Gardanne, est surtout bien représentée dans le répertoire des DS.P. (Pelletier et al., 1991, p. 316 ; Pelletier, 1997, p. 112-113). Des jarres (forme J) ont été retrouvées uniquement à Velaux (fig. 71, $\mathrm{n}^{\mathrm{o}} 6$ ) et au prieuré de SaintJean-de-Garguier à Gémenos ; leur rareté peut s'expliquer par la concurrence des amphores. Pour mémoire, la jatte forme N n'a été identifiée qu'à Saint-Blaise (fig. 70, no 2). 

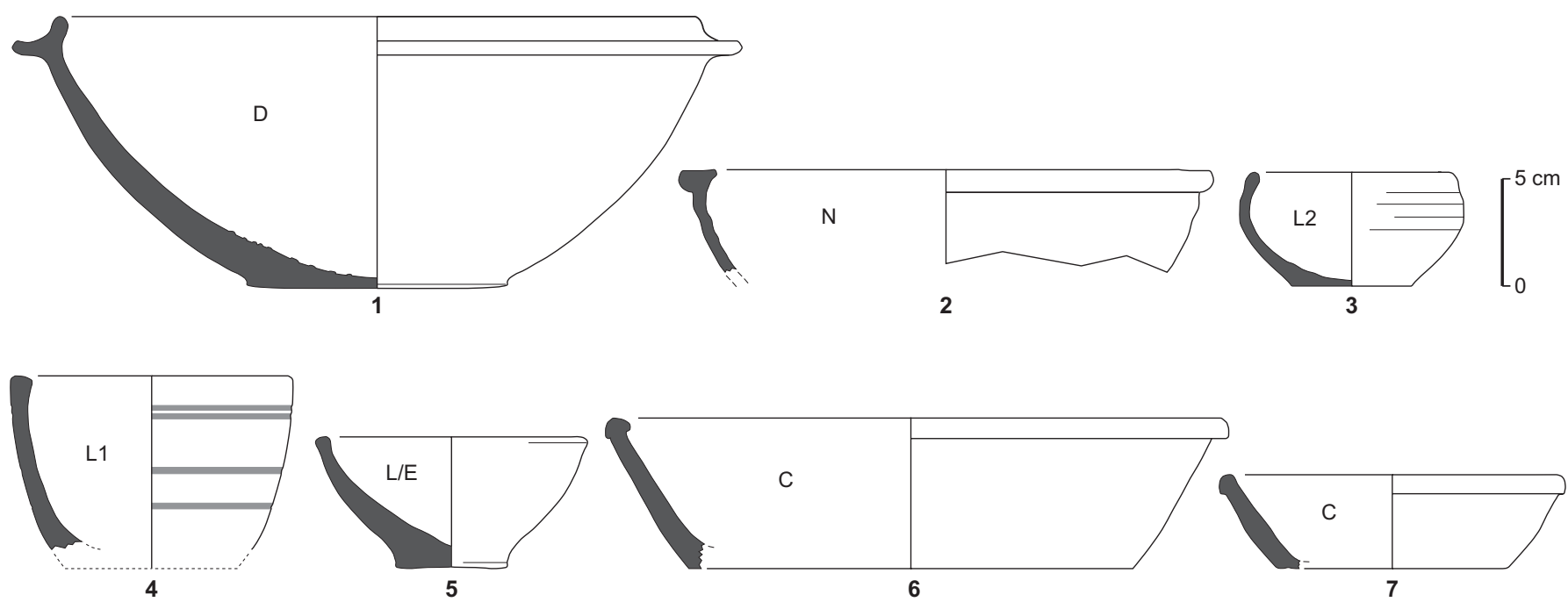

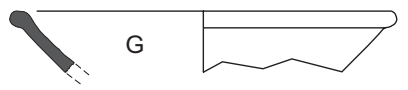

8
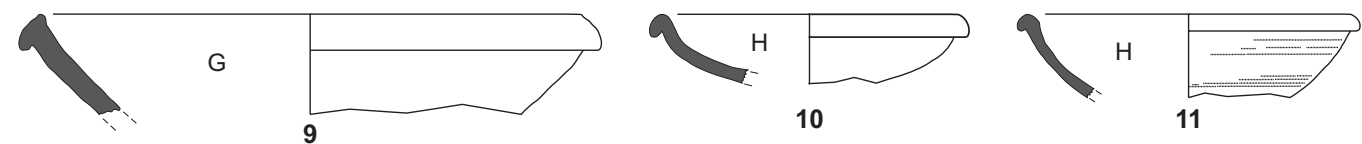

Fig. 70 - Typologie des formes ouvertes plus ou moins rares des céramiques à pâte grise provençales. Origine des exemples présentés : 1, 5, Gardanne ; 2-4, Saint-Blaise; 6-11, Apt (DAO : J.-P. Pelletier, CNRS).

Les plats de forme $\mathrm{C}$ (fig. $70, \mathrm{n}^{\mathrm{os}} 6$ et 7 ), dont le fond très large contraste avec l'étroitesse des fonds habituels, ne se retrouvent que dans quelques contextes du $\mathrm{V}^{\mathrm{e}} \mathrm{s}$. Les coupes et coupelles de forme $\mathrm{G}$ et $\mathrm{H}$ (fig. 70, $\mathrm{n}^{\text {os }} 8$ à 11), comme les formes B9 (fig. 69, no 16), ont été produites uniquement dans les ateliers de la région d'Apt et diffusées en quelques exemplaires à la fin du $\mathrm{V}^{\mathrm{e}} \mathrm{s}$. et au $\mathrm{VI}^{\mathrm{e}} \mathrm{s}$. vers le sud, jusqu'à Eyguières notamment et dans la région aixoise.

Dans son état actuel, cette typologie comprend la plupart des formes assez bien définies, mais un certain nombre d'éléments trop fragmentaires ou découverts très ponctuellement, difficiles à rapprocher des formes les plus répandues, ne sont pas évoqués. De nouvelles fouilles devront permettre de mieux préciser, d'une part la chronologie de l'apparition des céramiques communes grises, et d'autre part les séquences de la fin de l'Antiquité tardive encore mal connues à partir de la seconde moitié du $\mathrm{VI}^{\mathrm{e}} \mathrm{s}$.

J.-P. P.

\section{LES CÉRAMIQUES COMMUNES BRUNES DE PROVENCE ORIENTALE}

Lors des fouilles de Vintimille fut identifiée une céramique à pâte brune de l'Antiquité tardive (Lamboglia,
1950). La découverte d'une série de ratés de cuisson semblait indiquer une production locale. Une première typologie et plusieurs groupes de pâtes furent définis à partir d'analyses pétrographiques (Olcese, 1989 et 1993). Lors de l'étude du mobilier tardif de l'oppidum de Saint-Blaise, ont été mises en évidence de petites quantités de céramiques culinaires à pâte rouge qualifiées de "production italique, ligure et/ou indeterminée » (Vallauri, 1994). Des analyses réalisées par M. Picon ont montré qu'une partie de ces objets avait la même composition que ceux de Vintimille. Cependant, il n'a pas été possible d'attribuer une provenance certaine au reste du mobilier à la texture plus grossière, mais apparenté par la typologie aux objets de la catégorie précédente.

Une origine ligure a été retenue par le groupe CATHMA dans une typologie où les céramiques brunes apparaissent parmi diverses productions africaines et orientales (CATHMA, 1991 ; Raynaud, 1993a) sous l'appellation collective de «commune méditerranéenne tardo-antique » (com-medit). Différents travaux ont montré parallèlement que les céramiques brunes représentent la plus grande partie des céramiques communes durant l'Antiquité tardive, à Toulon (Bérato et al., 1986), Antibes (Güll, 1993), Nice/ Cimiez (Galassi, 1999) et, en règle générale, dans les départements du Var et des Alpes-Maritimes. Elles apparaissent 

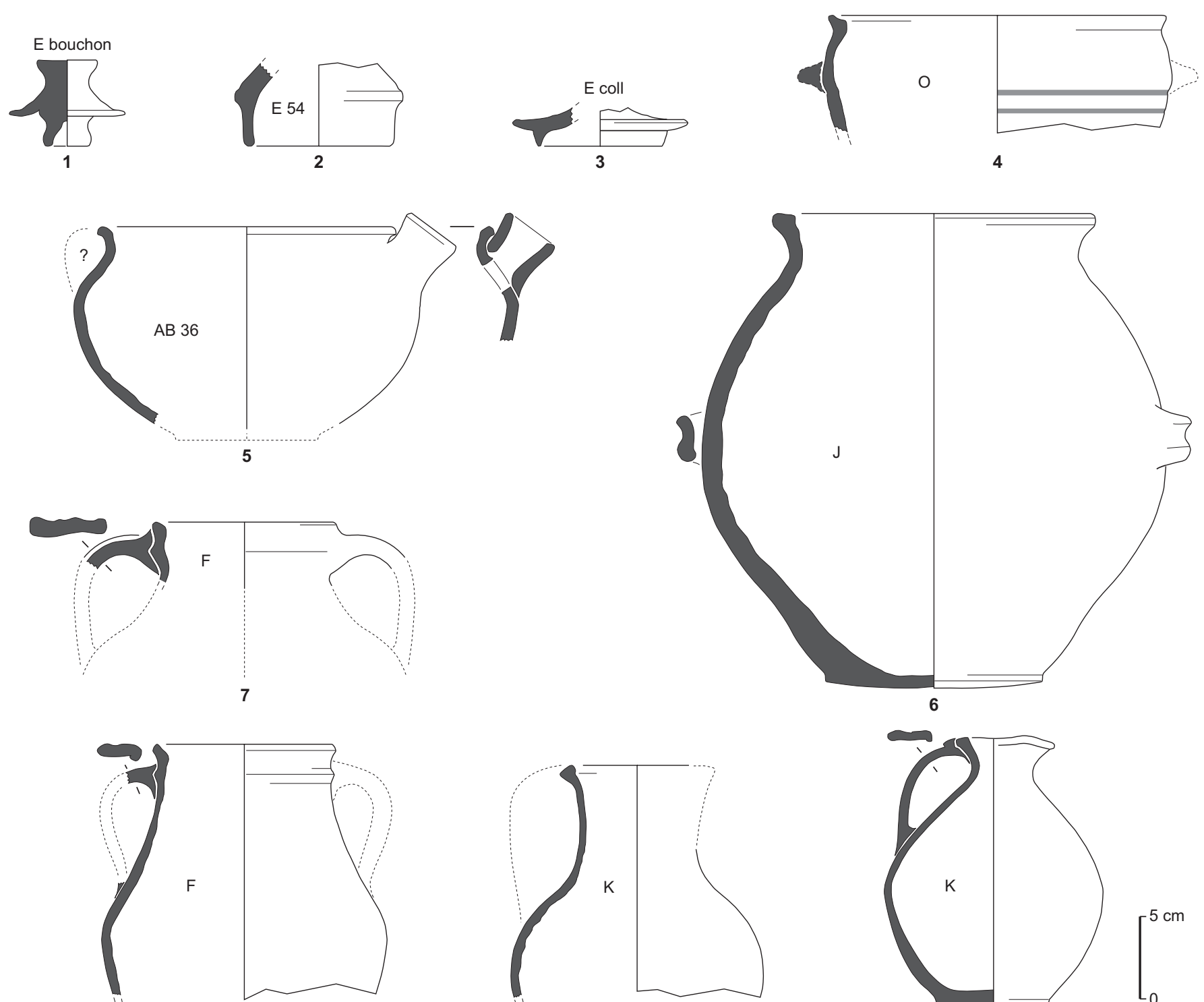

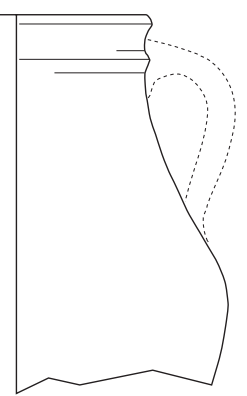

8

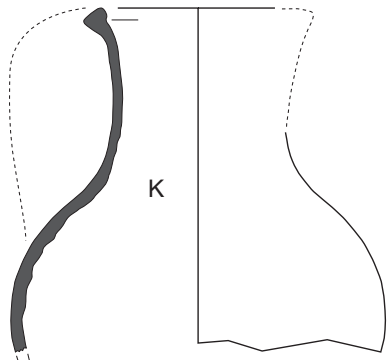

9

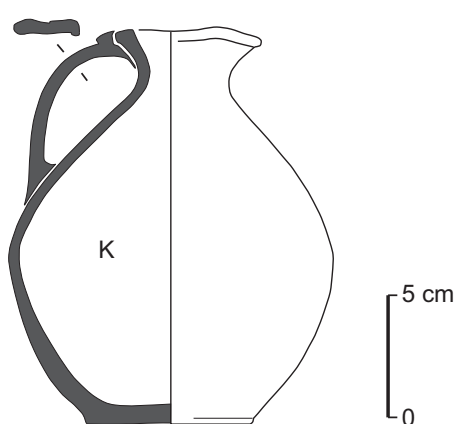

10

Fig. 71 - Typologie des formes rares "fermées » des céramiques à pâte grise provençales. Origine des exemples présentés : 1, 6, 8, Velaux; 2, 5, 9, Gardanne ; 3, Saint-Blaise ; 4, Marseille, Phocéens ; 7, Marseille, Saint-Victor ; 10, Marseille, Bon-Jésus (DAO : J.-P. Pelletier, CNRS).

le plus souvent associées à du mobilier des $\mathrm{V}^{\mathrm{e}} \mathrm{s}$. et $\mathrm{VI}^{\mathrm{e}} \mathrm{s}$, mais sont déjà présentes au milieu du $\mathrm{IV}^{\mathrm{e}} \mathrm{s}$. à Notre-Damed'Avinionet à Mandelieu-la-Napoule (Rivet, 1990) et à la fin du même siècle à Fréjus (Béraud et al., 1991) et à Olbia (Tréglia, 2001).

La Provence a donc pu apparaître divisée en deux, les Alpes-Maritimes et le Var dépendant d'importations ligures tandis que les Bouches-du-Rhône restaient le domaine des céramiques à pâte grise. Cependant, comment expliquer cette dépendance de la Provence orientale durant l'Anti- quité tardive, compte tenu de l'importance des productions régionales du Haut-Empire, notamment à Fréjus, dans la vallée de l'Argens et, dans une moindre mesure, entre Cannes et Mandelieu-la-Napoule, productions massivement distribuées en Ligurie?

La diversité des pâtes, des traitements de surface et du répertoire des céramiques à pâte brune de l'Antiquité tardive ont permis de s'interroger sur l'idée d'une source d'approvisionnement unique. La découverte de ratés de cuisson d'objets entrant dans le répertoire de Vintimille à 
Mandelieu (Rivet, 1982) et à Cagnes-sur-Mer (Pellegrino, 2003) a remis en question l'hypothèse d'une origine ligure exclusive. Il est assez difficile de définir cette catégorie en raison de la diversité de son aspect. Il est rare que l'on retrouve des produits semblables d'un site à l'autre et des objets identiques sur le même site. On a l'impression d'une production peu normalisée, provenant de plusieurs ateliers à la diffusion limitée, mais liés par des techniques et des traditions communes. Il s'agit d'une céramique granuleuse, cuite en atmosphère oxydante. Les parois sont minces. Le répertoire comprend une majorité de petits pots à feu pansus à fond lenticulaire et deux courtes anses verticales, différents types de coupes ou faitouts, des couvercles, quelques mortiers à collerette et quelques rares formes fermées (fig. 72). Il faut noter que les mortiers à collerette ont aussi été fabriqués dans une pâte calcaire, parfois micacée, qui rappelle celle des productions de l'Argens du Haut-Empire.

En dehors des produits de Vintimille, majoritaires sur certains sites comme Cimiez (Grandieux, 2004), ou Olbia (Tréglia, 2001), il est possible de distinguer au moins trois groupes de production, celui de Mandelieu-la-Napoule, de Cagnes-sur-Mer et de l'abbaye du Thoronet. C'est surtout à Cannes-la-Bocca, au pied de la butte Saint-Cassien, que l'on trouve les produits de Mandelieu. Ils se distinguent par leur pâte assez grossière, très sableuse et riche en paillettes de mica, de couleur rouge orangée assez vive. Les formes les plus caractéristiques sont de petits pots à feu pansus. Leur bord est évasé, terminé par une lèvre en bourrelet légèrement saillant à gouttière interne pour accueillir un couvercle. L'autre forme caractéristique est une coupe à bord rentrant, aux parois côtelées, avec des languettes de préhensions horizontales. L'atelier de Cagnes-sur-Mer a essentiellement produit deux types de pots à feu. Le premier est globulaire avec une lèvre déversée très développée et un ressaut interne. Le second possède une lèvre déversée, massive, de section triangulaire avec un ressaut interne, forme Cathma 13, qui appartient aussi au répertoire de Vintimille. La texture de la pâte est plus fine et plus homogène que celle de Mandelieu-la-Napoule, les parois beaucoup plus minces. Sa couleur est jaune orangé.

À l'abbaye du Thoronet, trois fours ont été identifiés dans un contexte du $\mathrm{V}^{\mathrm{e}}-\mathrm{VI}^{\mathrm{e}} \mathrm{s}$. Les produits associés, dont de nombreux rebuts de cuisson, sont irrégulièrement gris, bruns, orangés ou beiges. Le répertoire est apparenté à celui des céramiques communes à pâte grise de Provence occidentale (Pelletier, 1997). D'après J.-C. Tréglia, on retrouve ces productions à Olbia. Des objets, indifféremment bruns ou gris, sont courants tout particulièrement dans le Var, à Taradeau (Bérato, 2003), mais aussi au Montet à Gourdon (Pellegrino, 2002). Cela rend délicate l'identification du mobilier qu'on hésite souvent à ranger dans une catégorie, entre la «céramique commune liguro-provençale de l'Antiquité tardive ", préférentiellement cuite en atmosphère oxydante et les «céramiques communes grises provençales » (Pelletier et al., 1993), contemporaines et au répertoire très proche.

E. P.

\section{LA POTERIE KAOLINITIQUE RHODANIENNE}

Les données abondent en ce qui concerne les grandes productions kaolinitiques rhodaniennes et leurs ateliers, tant de la rive droite, ceux de l'Uzège, que de la rive gauche, tels les ateliers de Bollène et de Dieulefit. Leur production est intense dès le $\mathrm{V}^{\mathrm{e}} \mathrm{s}$., alors que naît l'un des grands centres de production potière qui dominera l'approvisionnement du Midi jusqu'à la fin du Moyen Âge et plus tard encore (Thiriot, 1986) (fig. 73). Dans le Languedoc rhodanien, de Bagnols-sur-Cèze à Beaucaire, la poterie kaolinitique concurrence dès lors la vaisselle à pisolithes, avec un répertoire typologique analogue. En Languedoc, la poterie kaolinitique prend progressivement le dessus au vie s. pour occuper une position hégémonique aux siècles suivants (CATHMA, 1993). Alors seulement, à partir du VII ${ }^{\mathrm{e}} \mathrm{s}$, s'opère la «médiévalisation » de l'équipement culinaire.

Caractérisée autant par sa typologie que par sa pâte blanche à surface noire ou grise craquelée bleutée, la poterie kaolinitique rhodanienne occupe une position singulière, encore mal cernée en l'état des recherches. Dominante dans la basse vallée du Rhône, au moins d'Avignon à Arles, elle s'étend vers le nord jusqu'à Lyon, où elle est présente en faible quantité (3\%) dans des niveaux du VII ${ }^{\mathrm{e}} \mathrm{s}$. (Horry, 2000, fig. 4, no 32). Sans qu'il soit possible, à ce jour, de retracer précisément la diffusion des grands ateliers cités plus haut, l'aire de répartition des produits en argiles kaolinitiques est néanmoins désormais mieux connue dans la vallée du Rhône moyen, entre Pierrelatte et Valence, grâce au panorama offert par les fouilles préventives sur les tracés de l'autoroute A49 et du TGV Méditerranée. Dans la seconde moitié du III ${ }^{\mathrm{e}}$ s., ils représentent près de $90 \%$ de la vaisselle commune dans la région de Crest et encore $54 \%$ deux siècles plus tard, alors que se fixent les principales formes du vaisselier du haut Moyen Âge. Plus au nord, dans la région de Valence, mais à la même période, la céramique kaolinitique le cède à d'autres catégories de vaisselles communes grises et rouges (Bonnet, Batigne-Vallet, 2001, p. 85, tabl. 3). 

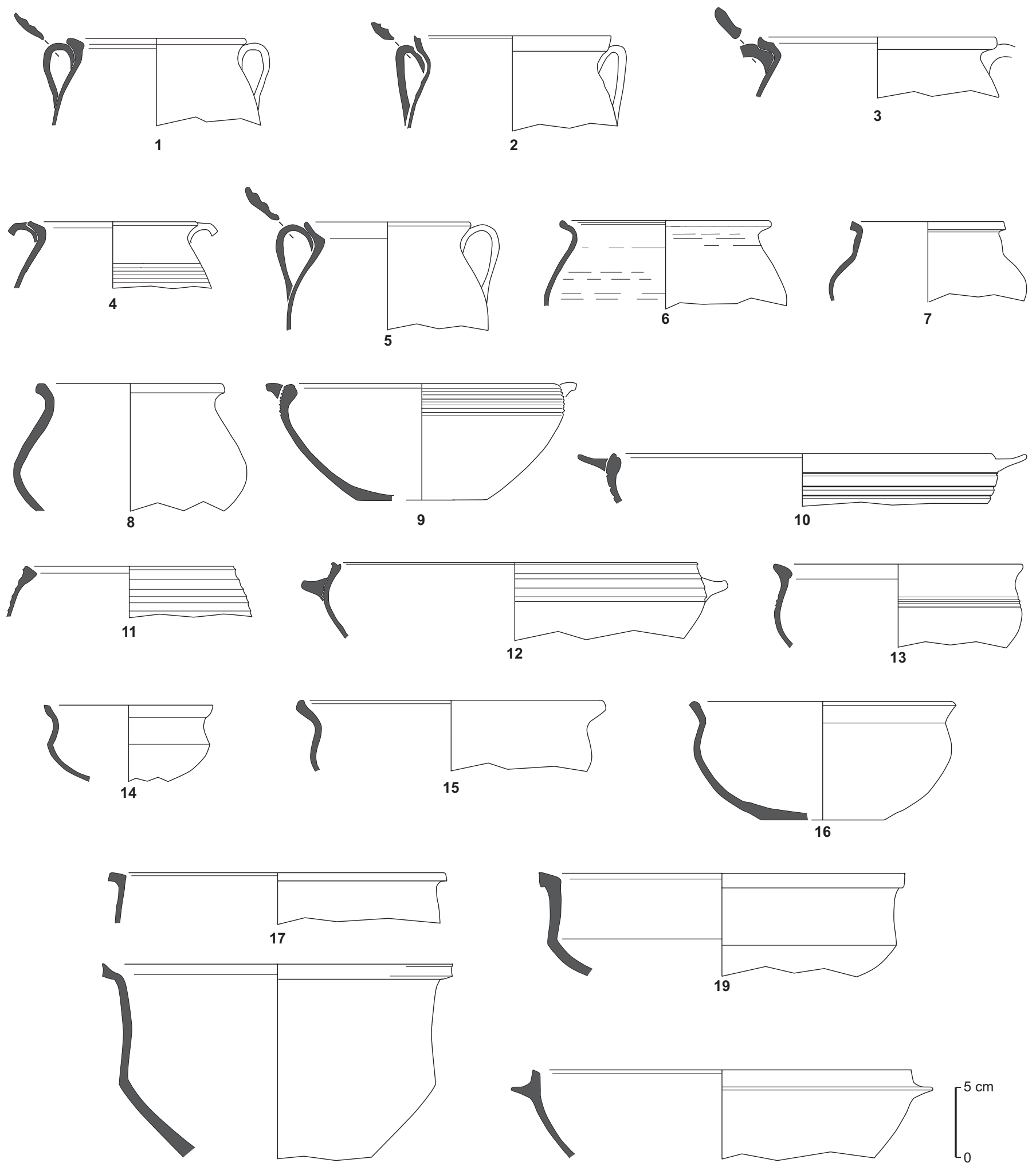

18
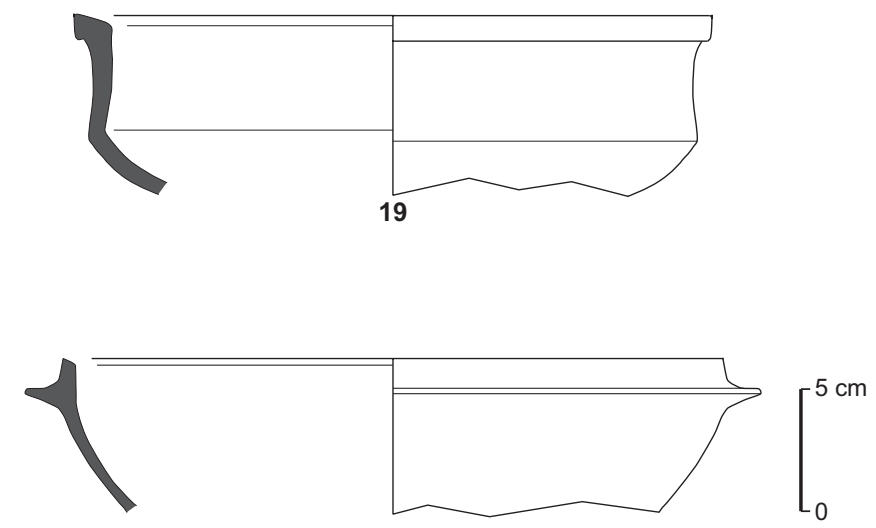

20

Fig. 72 - Exemples de formes significatives en céramique brune tardo-antiques de Provence-Orientale : 1, 5, 11, 13, atelier Saint-Véran à Cagnes-sur-Mer ; 2, le Montet à Gourdon ; 3, 7, 12, 14, 15, 17, hôtel des Mèdes, Île de Porquerolles à Hyères ; 4, église paroissiale de Beaulieu-sur-Mer ; 6, Vaugrenier à Villeneuve-Loubet ; 8, 19, villa des arènes de Cimiez à Nice ; 9, 18, Notre-Dame-d'Avinionet à Mandelieu-la-Napoule; 10, stade de Saint-Cassien à Mandelieu-la-Napoule; 16, 20, l'Ereste à Vence. 

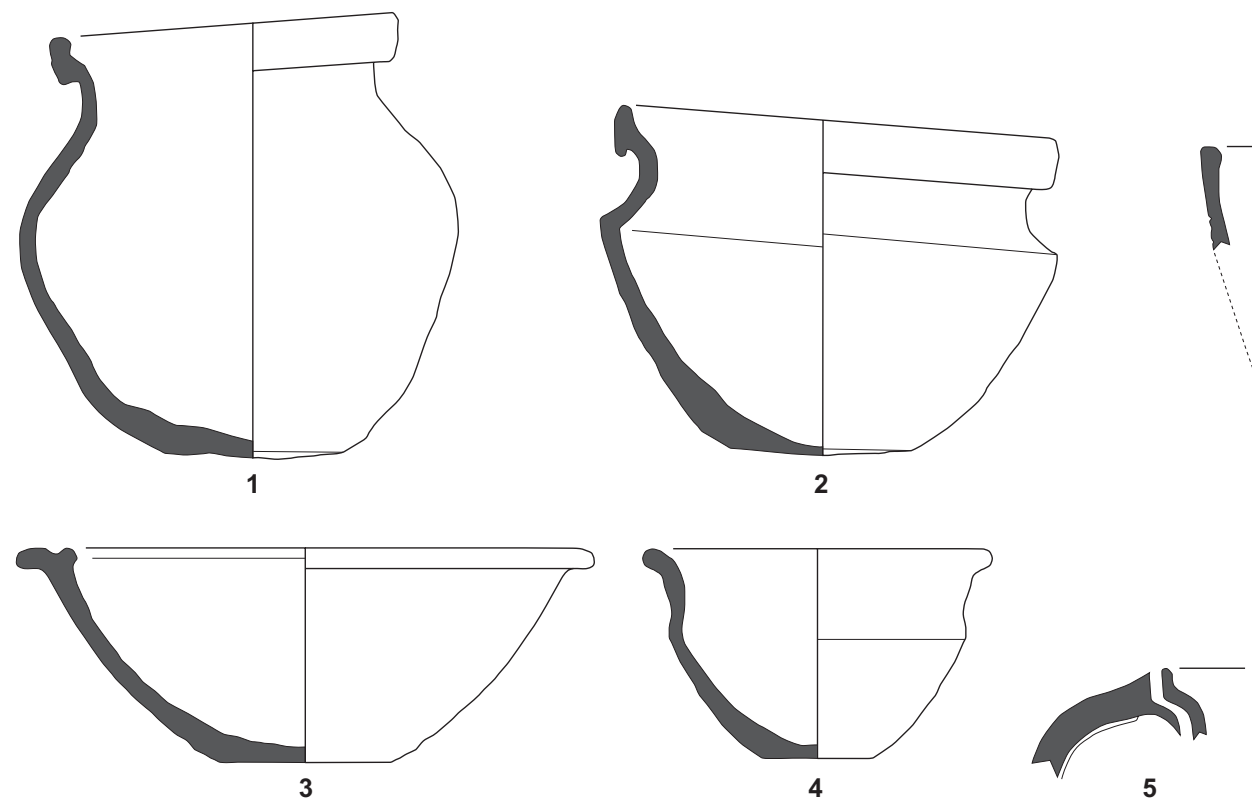

5

.+

Fig. 73 - Typologie des céramiques kaolinitiques de Bollène, Jonqueirolle (d'après Thiriot, 1986 ; DAO : M. Ott, INRAP).

Les observations faites sur les sites du haut Moyen Âge, dans le même secteur, sont tout aussi éloquentes. Composant l'intégralité de la vaisselle commune à Lapalud, moins de $10 \mathrm{~km}$ au sud de Pierrelatte, durant les $\mathrm{VII}^{\mathrm{e}}$-VIII $\mathrm{e}$., les poteries kaolinitiques sont encore présentes à $90 \%$ à Montboucher-sur-Jabron, à la latitude de Montélimar, entre le $\mathrm{VI}^{\mathrm{e}} \mathrm{s}$. et le $\mathrm{VIII}^{\mathrm{e}} \mathrm{s}$. Leur proportion faiblit régulièrement en remontant vers le nord. Ainsi, à Saint-Martin (Chabrillan), à une trentaine de kilomètres, elles représentent $53 \%$ des céramiques communes dans les niveaux des $\mathrm{V}^{\mathrm{e}}-\mathrm{VI}^{\mathrm{e}}$ s. et $70 \%$ dans les niveaux des $\mathrm{VI}^{\mathrm{e}}-\mathrm{VIII}^{\mathrm{e}}$ s. (Horry, 2006, tabl. XIII). Pour cette période, comme dans l'Antiquité, le secteur de Valence et la vallée de l'Isère semblent bien marquer la limite des scores élevés des produits à base d'argile à kaolinite: l'habitat hautmédiéval du Bivan à L'Albenc, en rive droite de l'Isère, livre moins de $20 \%$ de vaisselle kaolinitique, mais signale leur pénétration vers l'est, dans la vallée, pratiquement jusqu'à l'ombilic de Moirans (Faure-Boucharlat dir., 2001, p. 337-339).

Présente à peu près partout dans le Midi, mais souvent en faible quantité, cette vaisselle semble connaître une diffusion ramifiée à l'extrême, coiffant l'ensemble des productions régionales, pisolithes, Pabiran, grise provençale kaolinitique, sans pourtant les supplanter avant le $\mathrm{VII}^{\mathrm{e}} \mathrm{s}$. Une telle répartition s'explique-t-elle, par exemple, par l'accompagnement d'autres produits, alimentaires? Voilà un paradoxe qu'il faudrait mieux cerner afin de comprendre des processus commerciaux qui nous échappent actuellement.

C. R. et E. B.

\section{DIVERSITÉ DES PRODUCTIONS, HOMOGÉNÉITÉ DU FACIÈS ALPIN}

Quatre études consacrées respectivement au mobilier de l'atelier de Portout, à l'habitat de hauteur de Châteld'Arrufens (canton de Vaud), à l'établissement de plaine du Bivan à L'Albenc, aux pays du Lyonnais et enfin au quartier Saint-Jean à Lyon permettent d'esquisser un premier bilan sur la région rhodano-alpine, du Léman à Lyon et à l'Isère (Pernon, Pernon dir., 1990 ; Ayala, 1996 et 1998 ; FaureBoucharlat dir., 2001 ; David-Elbiali, Paunier dir., 2002). Sélectionnées pour la qualité de leur présentation autant que pour leur localisation géographique, ces quatre études font apparaittre, entre le milieu du IV ${ }^{\mathrm{e}}$ s. et le milieu du V $\mathrm{V}^{\mathrm{s}}$., une certaine homogénéité des productions. Le terme est à dessein mis au pluriel dans la mesure où tout incline à envisager de multiples provenances. Par exemple, au IV ${ }^{\mathrm{e}} \mathrm{s}$., le seul établissement du Bivan révèle sept productions distinctes provenant de plusieurs officines (Ayala, 1996, p. 262). Cette considération n'enlève, pour autant, rien à la convergence révélée par la typologie des poteries et par leurs caractères techniques. Ces derniers font apparaître la 
domination de la céramique commune, parfois nommée « grossière » (David-Elbiali, Paunier dir., 2002, p. 125), à pâte sableuse, dure et rugueuse, avec deux variantes imputables au mode de cuisson, oxydante pour les pâtes rouges ou brunes, réductrice pour les pâtes grises ou noires, dont la typologie apparaît voisine de celle des productions méridionales (Ayala, 1998, fig. 25-26).

Laissant à une enquête ultérieure le soin de mieux caractériser les faciès de cette vaste région, considérons globalement les grandes tendances de la vaisselle commune. Celle-ci fait apparaître nombre de traits communs dans la typologie comme dans la morphologie des différentes productions dominées, comme au Haut-Empire, par les formes ouvertes, assiettes tronconiques et marmites à panse hémisphérique, majoritairement caractérisées par des bords en amande, tandis que s'impose le mortier à listel. Les ollae perpétuent elles aussi le type ancien à panse ovoïde, tout en se distinguant par des bords en amande (David-Elbiali, Paunier dir., 2002, p. 213), ou par des bords en bourrelet (id., p. 212 ; Pernon, Pernon dir., 1990, pl. X). L'échantillon représentatif provenant de l'établissement du Bivan illustre la diversité des dispositifs de cols et rebords en cette seconde partie du IV s. (Ayala, 1996, fig. 3)

Si les formes évoluent peu au cours de ce siècle, une tendance se fait jour dans le choix du mode de cuisson avec la progression sensible des poteries grises; faisant presque jeu égal à Lyon au $\mathrm{IV}^{\mathrm{e}} \mathrm{s}$. $(35,6 \%$ de poterie noire contre $30,3 \%$ de poterie rouge), les poteries réductrices deviennent ensuite majoritaires tandis que les productions rouges stagnent autour de $30 \%$ (Ayala, 1998, p. 231). Plus au sud et en milieu rural, dans les rejets domestiques du Bivan, pâtes grises et sombres représentent $96 \%$ de la vaisselle commune (Ayala, 1996, fig. 2).

On peut désormais suivre l'évolution du contexte au-delà de la période antique, pour une vaste partie de l'arc rhônealpin, du Léman genevois et vaudois à la région lyonnaise, en passant par les pays du haut Rhône et la vallée du Rhône moyen, depuis l'Isère jusqu'à la Drôme. Les études récentes montrent que le début du Moyen Âge voit, d'une certaine manière, se renforcer les traits d'un «faciès rhône-alpin ", non tant à travers les modes de cuisson, critères dont on verra les limites ci-dessous, qu'à travers l'homogénéisation et la simplification morphologique du vaisselier.

C'est entre la fin $d u V^{e} s$. et la fin du VII ${ }^{e} s$. que la vaisselle grise gagne définitivement son hégémonie, mais avec des décalages régionaux très marqués. Si la poterie est uniformément grise dès le début $d u \mathrm{~V}^{\mathrm{e}} \mathrm{s}$. à Châtel-d'Arrufens dans le secteur vaudois, le processus est à la fois plus tardif et plus lent dans le secteur lyonnais. Ainsi, à Trévoux, à une vingtaine de kilomètres au nord de Lyon, la céramique cuite en atmosphère oxydante, en l'occurrence la production dite pâte bistre (voir supra, p. 107-109), représente $80 \%$ du vaisselier à la charnière des VI ${ }^{\mathrm{e}}-\mathrm{VII}{ }^{\mathrm{e}} \mathrm{s}$. (FaureBoucharlat dir., 2001, p. 227, fig. 4), tandis qu'à Lyon même (sites des Célestins, de la rue Pierre-Audry et de la rue des Chartreux), à partir du milieu du VII ${ }^{\mathrm{e}}$ s., la céramique en cuisson réductrice l'emporte régulièrement: entre $60 \%$ et $80 \%$ (Horry, 2000, p. 18-23 ; Faure-Boucharlat dir., 2001, p. 66, fig. 20 ; Ayala et al., 2003, p. 39-40, fig. 4-5). Mais les observations faites en Lyonnais ne sont pas transposables en aval de Valence, voire de Saint-Vallier: sur les habitats $\mathrm{du} \mathrm{V}^{\mathrm{e}}$ au VII ${ }^{\mathrm{e}}$ s., les rares céramiques communes non kaolinitiques sont exclusivement en pâte grise (Horry, 2006). Il apparaît bien que la diversité de ces situations est probablement à mettre davantage en relation avec les performances des argiles des sites d'approvisionnement qu'avec des faits d'ordre culturel.

On l'a dit plus haut, partout, depuis la fin du $\mathrm{IV}^{\mathrm{e}}$ s., la vaisselle culinaire est dominée par les pots ovoïdes ou ollae. Celles-ci connaissent une évolution assez sensible durant le $\mathrm{V}^{\mathrm{e}} \mathrm{s}$. : les panses évoluent vers un profil globulaire, les cols sont moins marqués et les profils des lèvres perdent de leur diversité au profit d'une certaine standardisation, à travers l'adoption du rebord en bandeau (ou en poulie) à profil anguleux (Ayala, 1998, p. 245, $\left.\mathrm{n}^{\text {os }} 174-176\right)$. Les formes ouvertes, quant à elles, perpétuent un répertoire commun à l'ensemble des régions rhodaniennes, jusqu'à la Méditerranée : gobelets tronconiques, bols carénés à lèvre en amande, mortiers hémisphériques à collerette (fig. 57, nº 3).

L'apparition du rebord en bandeau et son succès immédiat dans toute la région considérée dès le milieu $\mathrm{du} \mathrm{V}^{\mathrm{e}} \mathrm{s}$. constituent un phénomène à souligner. Tout en évoluant et en se diversifiant, épais et court (première génération), puis mince et plus haut (deuxième génération), il équipera pratiquement toute la vaisselle culinaire, claire ou sombre, du VI ${ }^{\mathrm{e}}$ au VIII ${ }^{\mathrm{e}}$ s., du Léman aux portes de la Provence et du Languedoc (Faure-Boucharlat dir., 2001, p. 69-72 ; Haldimann, 1994, p. 57-58 ; Horry, 2006). Quant au répertoire des formes alors disponibles, si la vaisselle de table résiste relativement bien jusqu'au milieu du VII ${ }^{\mathrm{e}}$ s., surtout en contexte urbain (Lyon, Célestins et rue Pierre-Audry, état 1) où elle occupe près de la moitié des ensembles étudiés, sa chute est rapide au-delà de ce seuil (17\% à $27 \%$ ), à la ville comme à la campagne : les jattes et mortiers disparaissent en premier, puis les bols carénés, plus ou moins remplacés par des gobelets étroits, cylindri- 
ques, galbés ou tronconiques, aux parois souvent striées, qui, aux côtés des pots globulaires et de rares cruches, sanctionnent définitivement la «médiévalisation " du vaisselier (Horry, 2000, tabl. 12 ; Faure-Boucharlat dir., 2001, p. 69, fig. 23 ; Ayala et al., 2003, fig. 9 ; Horry, 2006).

Diversité des productions mais homogénéité du faciès, par les traits que l'on vient d'évoquer le « faciès » rhône-alpin partage les tendances générales observées dans l'ensemble de la Gaule méridionale même s'il reste alimenté par des ateliers régionaux. Pour l'approvisionnement de la ville de Lyon, par exemple, plusieurs officines sont envisagées en Beaujolais et en rive de Saône, l'analyse permettant d'identifier, au sein du mobilier de Saint-Jean, au moins six pâtes distinctes, du milieu du $\mathrm{IV}^{\mathrm{e}} \mathrm{s}$. au V $\mathrm{V}^{\mathrm{e}}$ s. (Horry, 2000, p. 18). Au niveau régional, de nombreuses similitudes apparaissent dans la morphologie et les modes de cuisson, qui entrent dans une ambiance rhodanienne où la vaisselle grise de Châtel-d'Arrufens rappelle bien des traits observés sur la poterie kaolinitique de la région ardéchoise ou encore les productions à pisolithes de la région nîmoise.

Mais alors que disparaît la vaisselle brune rhodanienne, seule désormais la production du Val de Saône va perpétuer, durant un à deux siècles, la production des ollae, des bols carénés et des mortiers à pâte claire, dans la tradition antique.

C. R. et E. B.

\section{LE VERRE : UNE PRODUCTION HIÉRARCHISÉE}

Depuis la tenue en 1986 de l'exposition « Les premiers temps chrétiens en Gaule méridionale », la poursuite des études archéologiques et les nouvelles recherches archéométriques sur la verrerie de l'Antiquité tardive en Gaule du Sud ont permis :

- tout d'abord d'enrichir le catalogue du mobilier en verre en usage $d u \mathrm{IV}^{\mathrm{e}} \mathrm{s}$. au début $\mathrm{du} \mathrm{VIII}^{\mathrm{e}} \mathrm{s}$. (vaisselle, luminaire et verre architectural), de préciser quelques datations et surtout de distinguer quelques produits manufacturés importés ;

- de localiser quelques ateliers ;

- de retracer la chaîne de production du verre et d'observer l'évolution des techniques.

Le colloque "Le verre de l'Antiquité tardive et du haut Moyen Âge ( $\mathrm{IV}^{\mathrm{e}}-\mathrm{VIII}{ }^{\mathrm{e}} \mathrm{s}$.) » a été l'occasion de proposer des typochronologies de la verrerie du midi de la Gaule. Ces synthèses qui reposent sur de nombreuses études monographiques de la Provence et du Languedoc (Foy, 1995), mais aussi de l'Aquitaine (Foy, Hochuli-Gysel, 1995), pourraient aujourd'hui être confortées par une documentation nouvelle (ou nouvellement exploitée), mais dispersée et provenant principalement des sites littoraux d'Olbia (Fontaine, 2006, p. 365-371), de divers secteurs de Marseille (Foy, 1998) et d'Arles, de l'oppidum de Constantine (fouilles Gourvest et récemment F. Verdin), de Narbonne (fouilles de la Lombarde et de l'Hôtel-Dieu : Ginouvez dir., 1996-1997), de Loupian et du port du Bourbou et des épaves Port-Vendres I et la Redoute Béar. À l'intérieur des terres, les verreries des fouilles anciennes et récentes de la région Midi-Pyrénées et des territoires limitrophes (divers chantiers de Toulouse, Cahors, Rodez, mais aussi sites de La Valentine, Saint-Bertrand-de-Comminges, Eauze, L'IsleJourdain, Chastel-sur-Murat, Arpajon-sur-Cèze, Lapanousede-Cernon) font aujourd'hui l'objet de travaux universitaires (Cornardeau, 2004-2005). Les fouilles menées à Lyon (Foy, 2006), à Milhaud et, plus anciennement, sur l'habitat perché de Larina à Hières-sur-Amby, fournissent aussi un mobilier significatif.

En suivant l'ordre chronologique, nous pouvons présenter les nouveaux apports en insistant plus particulièrement sur le mobilier importé qui comprend des produits manufacturés et du verre brut.

Bien que le répertoire typologique de la verrerie employée durant le $\mathrm{IV}^{\mathrm{e}} \mathrm{s}$. soit moins bien connu que celui des deux siècles suivants, nous pouvons assurer que les formes les plus communes sont les grands verres cylindriques (Isings, 1957, forme 109a) que l'on rencontre par exemple dans les nécropoles de l'Esplanade à Arles et Saint-Michel à Montpellier (Majurel et al., 1970-1973). Les gobelets ovoïdes et les coupes au rebord coupé sans lèvre, soufflés dans une matière claire, légèrement verdâtre ou bleutée, composent l'essentiel des services de verres de la fin $\mathrm{du} \mathrm{IV}^{\mathrm{e}} \mathrm{s}$. Ces formes perdureront plusieurs décennies, mais seront alors fabriquées dans une autre matière.

À côté de cette vaisselle commune existent des verres décorés qui constituent la documentation la plus neuve.

La gravure, procédé décoratif le plus fréquent à la fin du $\mathrm{IV}^{\mathrm{e}} \mathrm{s}$. ou du début du siècle suivant, apparaît principalement sur des gobelets tronconiques, des bols et de larges coupes hémisphériques au rebord coupé, et plus rarement sur des bouteilles cylindriques.

Le thème, la syntaxe et les caractéristiques techniques du décor permettent de reconnaître plusieurs types de gravure et dans certains cas de proposer une origine. Les décors géométriques, ordonnés en registres horizontaux ou en médaillons 


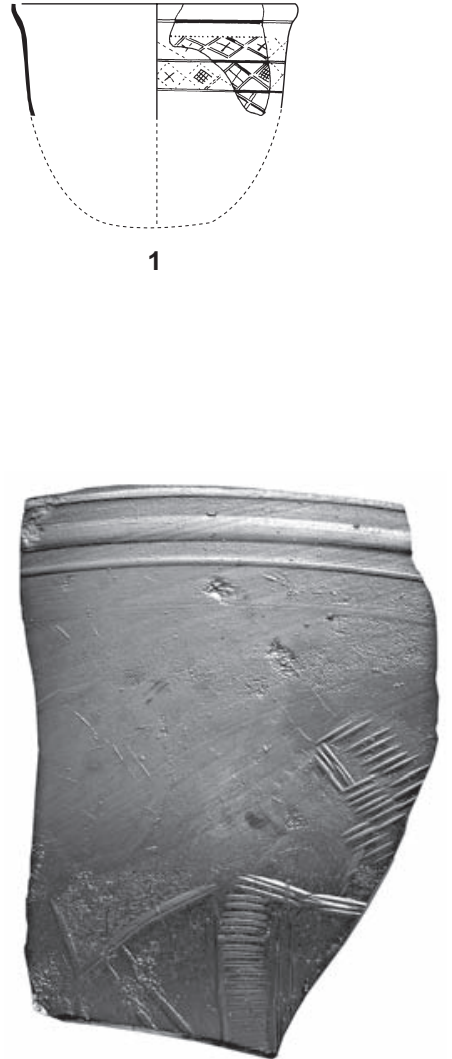

4
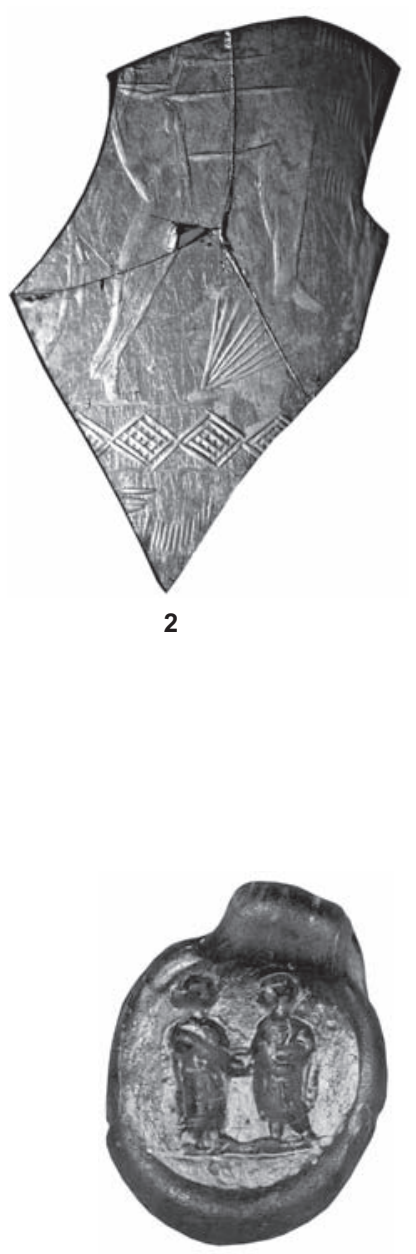

5

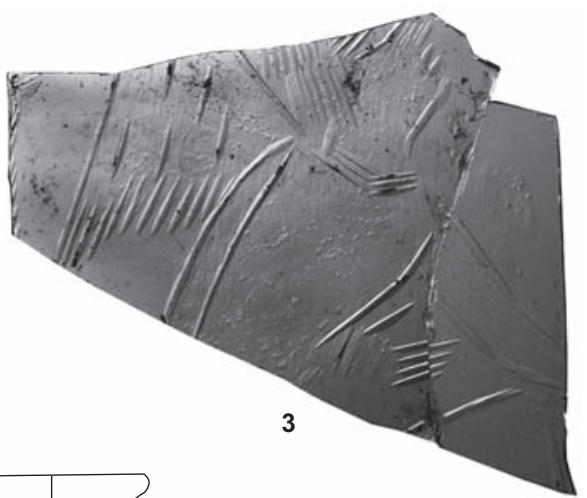

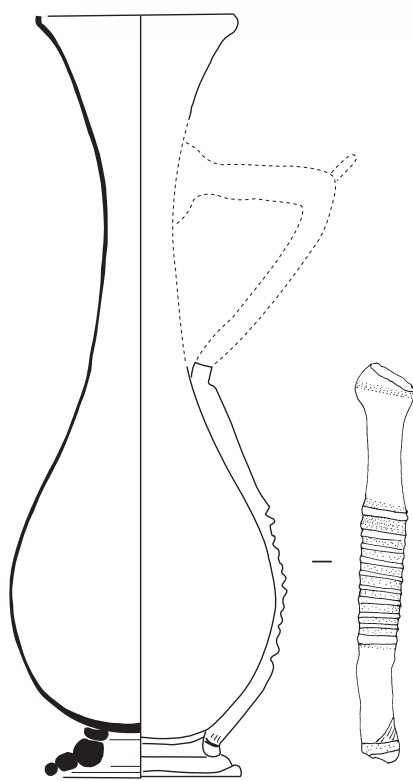

6

Fig. 74 - Production de verre : 1, rebord de gobelet à décor gravé, production régionale ?, Narbonne, Hôtel-Dieu (fouilles : O. Ginouvez, INRAP), début $V^{e}$ s. ; 2, fragment de gobelet à décor gravé, Gadagne, Lyon (fouilles : C. Becker, Service municipal de Lyon), cortège bachique, importation de Rome ?, début du $V^{e} s$., longueur maximale $7 \mathrm{~cm} ; 3$, fragment de bouteille à décor gravé, Milhaud (fouilles: F. Conche, INRAP), importation de Rome ?, début du $V^{e} s . ; 4$, fragment de coupe à décor gravé, Arles, fouilles anciennes, importation de Rhénanie ?; 5, médaillon estampé, nécropole de Lunel-Viel (fouilles : C. Raynaud), IV s., longueur maximale : 2,10 cm, importation syro-palestinienne; 6, cruche bleu cobalt, Marseille, basilique de la rue Malaval (fouilles : M. Moliner), seconde moitié du IV s. ?, importation de Méditerranée orientale $\left(n^{o s}\right.$ 1, 4, 6, dessins et cliché : D. Foy, CNRS; $n^{o}$ 2, cliché : Y. Rigoir, LAMM; $n^{0}$ 3, cliché : C. Durand, CNRS; $n^{o} 5$, cliché :

L. Damelet, CNRS).

au milieu du fond des objets, sont les plus habituels. Ces motifs très soignés, qui couvrent partiellement ou totalement la surface des verres, pourraient provenir d'ateliers régionaux (fig. 74, $\mathrm{n}^{\mathrm{O}} 1$ ). En revanche, sur d'autres pièces, subsiste un décor historié dont le thème et la facture évoquent des productions romaines ou plus rarement rhénanes : c'est le cas pour une coupe à décor chrétien de Narbonne dont l'iconographie très mutilée pourrait être complétée sur le modèle d'une pièce de San Lorenzo d'Ostie. Les scènes de pêche compartimentées dans des médaillons (Foy, Nenna dir., 2001, $n^{\circ} 409$ ), les cortèges dionysiaques trouvés sur plusieurs sites (Milhaud: Foy, Michel, 2003 ; Lyon, fouilles de Gadagne) (fig. 74, $\mathrm{n}^{\mathrm{o}}$ 2), la représentation de Dionysos appuyé sur son thyrse (Foy, Nenna dir., 2001, no 402-1), une bouteille avec un Éros (fig. 74, $\mathrm{n}^{\circ}$ 3), la coupe mettant probablement en scène Bellérophon et Pégase (fouilles de C. Pellecuer à Loupian, inédit) relèvent de styles différents les uns des autres, mais sont probablement les œuvres d'ateliers romains. En revanche, d'autres pièces provenant de la villa de Plassac (Foy, Hochuli-Gyssel, 1995, fig. 2-13), de l'hôpital Larrey, 
place Saint-Étienne à Toulouse (Cornardeau, 2004-2005, $\mathrm{n}^{\text {os }} 299$ et 376), d'Arles (fig. 74, $\mathrm{n}^{\circ}$ 4) et la dernière probablement de Dieulefit (Foy, Nenna dir., 2001, no 407), témoignent par leur iconographie et leur facture (taille dite paralleler Furchenschliff) de liens avec la Rhénanie.

Ces grandes coupes au décor très élaboré, d'inspiration mythologique ou chrétienne ou encore reproduisant des scènes de la vie quotidienne (pêche, chasse) étaient principalement signalées dans le nord de la Gaule (découvertes d'Amiens, Boulogne-sur-Mer, Abbeville, Vermand), en Suisse et surtout en Italie et en Rhénanie. Les découvertes anciennes de Carthage, ou plus récentes en Espagne, à Valence (Soriano Sánchez, 1992, p. 22-23) et à Barcelone (Beltrán de Heredia Bercero dir., 2001, p. 156-157), tout comme celles du midi de la France, montrent une plus large distribution, reflet de la circulation de ces verreries de grande valeur ou peut-être aussi témoignage de la diffusion des modèles (par exemple le thème de la traditio legis traité de manière très comparable sur la coupe de Valence et d'Obernburg-am-Main : Wamser, Zahlhaas dir., 1998, no 161) ou encore de l'itinérance des artistes graveurs puisque la gravure est la seule technique décorative à froid qui puisse se faire en dehors de l'atelier du verrier.

Les verres diatrètes taillés en très haut-relief, pièces de qualité exceptionnelle, très rares en Gaule, ne sont pas inconnus dans le Midi. La pièce découverte au $\operatorname{XIX}^{\mathrm{e}} \mathrm{s}$. à Arles (Chavigny, 1873) est malheureusement aujourd'hui disparue, mais deux fragments modestes trouvés dans le Sud-Ouest, sur les sites voisins de la villa de Séviac à Montréal (Hochuli-Gysel, 1993, fig. 3-8 et fig. 4) et de la domus de Cieutat à Eauze (Cornardeau, 2005, p. 50), confirment l'arrivée de produits de luxe fabriqués dans diverses officines occidentales et orientales.

Les importations de verres syro-palestiniens pourtant très fréquentes dans le Haut-Empire sont par la suite peu manifestes. Aussi la présence d'un médaillon estampé dans une tombe $d u I^{e}$ s. de Lunel-Viel est-elle surprenante (fig. 74, $\left.n^{\circ} 5\right)$; en effet, ces amulettes extrêmement communes en Palestine et en Syrie sont rarissimes en Occident (Collectif, 1990, n 116 ; Stern, 2001, p. 362-363). On signalera aussi la présence de cruches colorées en bleu cobalt et dotées d'une anse plissée et d'un pied formé par l'enroulement d'un cordon de verre rapporté. Ces supports caractéristiques ont été remarqués sur divers sites, en particulier sur l'oppidum de Saint-Blaise à Saint-Mitre-les-Remparts (Foy, 1994, p. 212-213, nos 124-126), à Arles (inédit) et à Marseille (fouilles de la Bourse : Foy, 1998, p. 132, fig. 97-209 et p. 191, fig. 162-225), mais seules les récentes explorations qui ont mis au jour la basilique de la rue Malaval à Marseille offrent une pièce presque complète, datée de la fin $d u I^{e} s$. par l'amphore qui la contenait (fig. 74, $\mathrm{n}^{\circ} 6$ ). Ces verreries sont vraisemblablement originaires de Méditerranée orientale (Israeli, 2003, p. 291).

Deux ateliers de verriers actifs dans le courant du $\mathrm{IV}^{\mathrm{e}} \mathbf{s}$. sont attestés : l'un, à Vienne, s'est installé dans une domus abandonnée à la fin du III ${ }^{\mathrm{e}}$ s. (Foy, Tardieu, 1986) ; l'autre, dans Arles sur le site de l'Esplanade, est identifié par de nombreux indices d'artisanat du verre concentrés dans des contextes datables de la seconde moitié du IV $\mathrm{s}$. (fig. 75, $\left.\mathrm{n}^{\text {os }} 1-3\right)$.

Le trait distinctif de la verrerie $d u \mathrm{~V}^{\mathrm{e}} \mathrm{s}$. est sa coloration sombre, vert olive ou jaune-brun, qui s'impose sans doute au cours du deuxième quart du siècle. Les formes déjà communément utilisées, gobelets et coupes à rebord sans lèvre, subsistent mais sont désormais réalisées dans cette nouvelle matière vitreuse. Le répertoire des formes composant cette catégorie comprend aussi des verres à pied, des coupes variées et plusieurs bouteilles ansées et flacons.

L'abondance particulière de ce type de verre en Égypte et ses caractéristiques chimiques incitent à considérer cette matière vitreuse comme originaire de cette province (Foy et al., 2003 ; Freestone et al., 2005). Dans l'Antiquité tardive comme aux époques précédentes, il ne semble pas exister en Gaule d'ateliers de verriers producteurs de matière vitreuse (ateliers primaires), mais uniquement des officines secondaires transformant le verre brut importé en objets manufacturés. De nombreux témoignages attestent que la matière brute de teinte olive a été mise en œuvre dans plusieurs officines secondaires, particulièrement à Marseille dans les quartiers suburbains de la cité comme le prouvent les découvertes de la Bourse : verre brut et déchets de soufflage, en particulier des mors évasés, calottes de verre qui sont les restes de verre attenants à la canne après découpage de l'embouchure de l'objet - ces mors ont naguère été publiés comme des embouchures de récipients (Foy, Bonifay, 1984, fig. 2, 26, 27 ; Foy, 1998, p. 167) - et les trouvailles plus récentes des chantiers de Jules-Verne et de l'Alcazar. Sur ces derniers sites comme à Arles (rue Truchet), les blocs de verre brut et les mors (fig. 75, $n^{\text {os }} 4-6$ ) constituent des éléments éloquents pour localiser des officines de verriers bien que les fours n'aient jamais été exhumés. D’autres blocs, mis au jour dans les places de transit (Bordeaux : fouilles de la place Camille-Jullian; Toulouse : fouilles du métro, voir fig. 75, $\mathrm{n}^{\circ} 7$; La Valentine : épave Port-Vendres I), attestent non pas la présence de centres artisanaux, mais la circulation des matières vers les ateliers secondaires. 


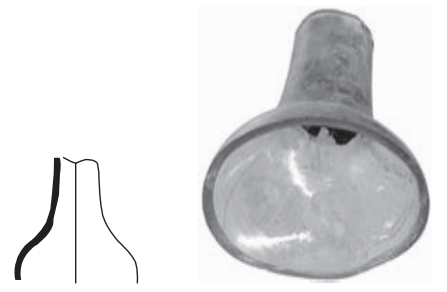

1
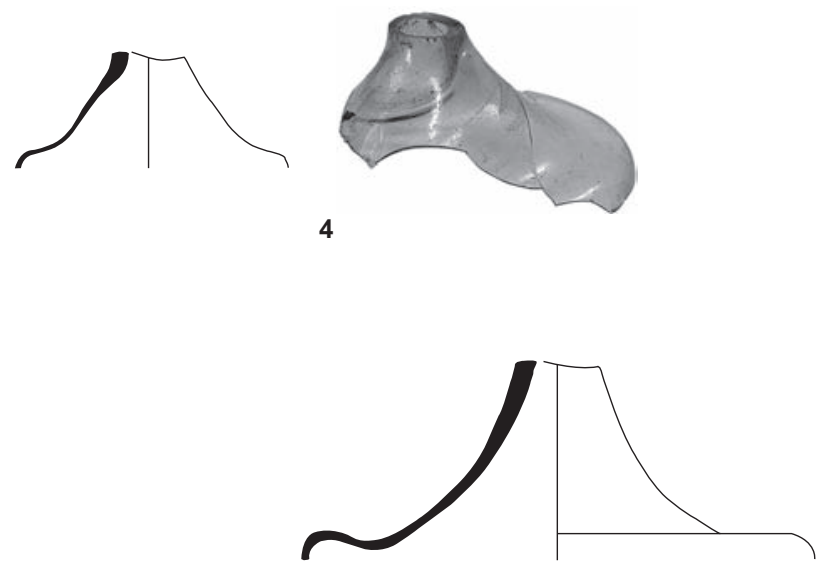

6
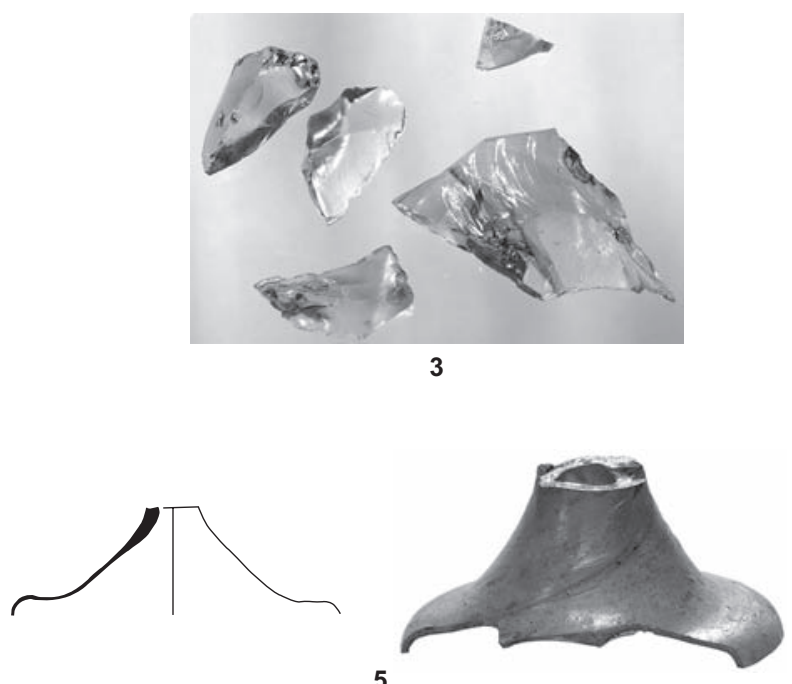

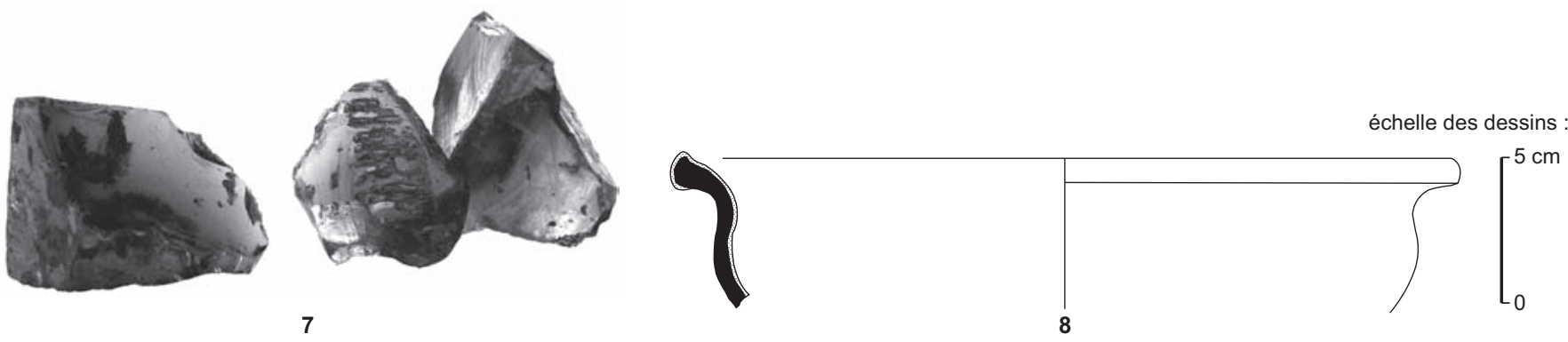

Fig. 75 - Production de verre : 1, 2, mors de verrier; déchets d'atelier, Arles, Esplanade (fouilles : MAPA, us 398), seconde moitié du IVe s. ; 3, éclats de blocs de verre brut incolore, déchets d'atelier, Arles, Esplanade (fouilles : MAPA, us 368), seconde moitié du IVe s. ; 4, mors de verrier, incolore verdâtre, Marseille, Alcazar (fouilles: M. Bouiron, us 13297), IV $V^{e} V^{e}$. ? ; 5, mors de verrier, vert-jaune, Marseille, place Jules-Verne, zone 10, us 73 (fouilles: A. Hesnard), Ves. ?; 6, mors de verrier, vert olive, Arles, rue Truchet (fouilles : MAPA), Ves. ?; 7, verre brut, vert olive, Toulouse, fouilles du métro (fouilles: INRAP), Ves.; 8, creuset de verrier, Marseille, la Bourse, aire 1, période 2A, phases 2 et 3, fin $V^{e} s$. (fouilles: M. Bonifay et Service régional de l'archéologie), Ves. (dessins et clichés : D. Foy, CNRS).

Les creusets de verriers sont aussi de bons indices pour repérer les officines secondaires; ces outils connus à partir $\mathrm{du} \mathrm{IV}^{\mathrm{e}} \mathrm{s}$. ne sont que des céramiques culinaires détournées de leur fonction domestique. Plusieurs formes sont connues, mais il s'agit toujours de jattes ou de coupes à large ouverture (Foy et al., 1990). Leur apparition signifie sans doute un changement de techniques : les fours à multiples creusets semblent se substituer aux fours à bassin maçonné formant un seul réceptacle pour la fonte du verre brut et du verre à recycler.

Une infime partie des blocs de verre brut n'était pas destinée à être refondue dans les officines mais à être débitée en tesselles pour les mosaïques. Sur plusieurs sites de l'Antiquité tardive et principalement dans les riches 
villae d'Aquitaine se retrouvent les produits semi-finis qui ont servi à la fabrication des mosaïques en verre. Les blocs de verre brut ne servaient qu'à obtenir des tesselles de verre incolore et translucide, mais la majorité des cubes de verre coloré et opaque provient de produits semi-finis qui voyageaient sous forme de galettes grossièrement circulaires ou ovales et de taille variable (de $10 \mathrm{~cm}$ à $40 \mathrm{~cm}$ de diamètre). Ces disques de verre, retrouvés en Italie, en Allemagne et en Gaule, sont peut-être originaires de l'Orient méditerranéen où ils sont bien attestés, mais d'autres provenances sont envisageables (Afrique, Espagne ?). La plupart sont colorés dans la masse en jaune, plus rarement en rouge et surtout par de multiples nuances de bleu et de vert; plus rares sont les plaques, non colorées, renfermant une mince feuille d'or (Saint-Sever; découvertes de P. Dubedat). Les disques ou les plaques de verre de couleur, pour l'instant reconnus à Châteauneuf-du-Rhône (fig. 76, no 2), à Marseille (fouilles de l'Alcazar), Arles (fouilles des thermes de Constantin), Rodez, Montmaurin et Séviac à Montréal (fig. 76, $\mathrm{n}^{\mathrm{o}} 1$ ) étaient utilisés pour le décor monumental (divers placages, inscriptions à la feuille d'or) ou même pour la joaillerie dans le cas d'un disque de verre très homogène.

Il est possible de distinguer au sein des verreries de teinte olive $d u V^{\mathrm{e}} \mathrm{s}$., découvertes en Provence et Languedoc, les pièces qui relèvent de productions régionales et celles qui ont été assurément ou probablement importées avec le verre brut. Au compte des importations égyptiennes, on peut mettre la vaisselle à décor géométrique abrasé ; ce procédé ornemental à peine visible étant relativement fréquent sur les bouteilles ansées cylindriques présentes dans les contextes du tout début du $\mathrm{V}^{\mathrm{e}} \mathrm{s}$. (fig. 77, $\mathrm{n}^{\mathrm{o}}$ 2) et plus rares sur les formes ouvertes (fig. 77, $\mathrm{n}^{\mathrm{o}} 1$ ) (Foy, 1995, p. 223). Les flacons bicéphales soufflés dans un moule (Marseille, Saint-Blaise et Narbonne: Foy, 1995, p. 222, $\mathrm{n}^{\text {os }} 17-19$; Foy et al., 2003, p. 54, no 274, voir ici fig. 77, no 3) et un verre à piédouche incisé (Marseille : Foy, 1995, p. 229, $n^{\circ} 107$, voir ici fig. $\left.77, n^{\circ} 4\right)$ ont vraisemblablement la même origine. Les importations orientales de vaisselle de verre en Méditerranée occidentale sont aussi visibles dans les fouilles de Tarragone qui ont livré quelques objets appartenant au répertoire des ateliers égyptiens (flacon à col resserré et bouteille et amphore cylindriques à décor abrasé : Benet i Arqué, Subias i Pascual, 1989, fig. 185 no 9.32, fig. 186, $n^{\circ} 9.43$ et fig. $187, n^{\circ} 9.47$ ).

Au nombre des importations de Méditerranée orientale, il faut rajouter des bouteilles ansées à panse prismatique dont le fond porte une inscription en grec. Rarement reconnus en Méditerranée occidentale (Calvi, 1968, p. 86, no 214 et fig. E), ces récipients retrouvés dans les fouilles urbaines de Nice et de Marseille (fouilles du tunnel de la Major, voir ici fig. 77, no 5 , et de la rue Lecca : Foy, Nenna dir., 2006, p. 195-200) viennent très certainement d'Asie Mineure (Tek, 2003). On notera que ces importations concernent presque toujours des récipients fermés et l'on a tout lieu de croire que ces verres n'ont pas été transportés pour eux-mêmes mais pour les denrées qu'ils contenaient.

La plus grande part des formes qui composent cette vaisselle olive, les coupes quelquefois dotées de dépressions sur la panse (fig. 77, $n^{\circ} 6$ ), les gobelets, parfois ornés de minces pastilles de verre bleu foncé appliquées (fig. 77, $\mathrm{n}^{\text {os }} 7$ et 8), et les verres à pied sont cependant des productions régionales réalisées à partir de verre brut importé d'Égypte. On notera que des pièces comparables (tant dans les profils, les décors et l'aspect de la matière vitreuse) se rencontrent aussi dans les productions de la mer Noire, mais pour l'instant aucun lien n'a été établi entre fabrications pontiques et méditerranéennes occidentales. Cette vaisselle à décor de pastilles bleues se différencie nettement des productions syro-palestiniennes et rhénanes qui sont réalisées dans un verre clair et plus épais. Rares sont les fragments en verre à peine verdâtre et décorés de pastilles bleues et vertes attestant de relations commerciales avec les centres de Cologne (une attestation dans le dépotoir portuaire de Port-Vendres I) ou du Levant (exemples provençaux d'Arles et de la villa du Viely à Cucuron, fouilles de l'Esplanade).

C'est au début $\mathrm{du} \mathrm{V}^{\mathrm{e}} \mathrm{s}$. que nous remarquons l'apparition des premiers panneaux de verre à vitre soufflé. Ce verre présente un aspect très différent de celui que l'on a coutume de rencontrer dans les habitats gallo-romains du HautEmpire. Beaucoup plus fins, ces fragments de verre ont été soufflés en cylindre et présentent deux surfaces parfaitement lisses. Plusieurs fragments proviennent des fouilles de la Bourse à Marseille et surtout des sites languedociens : Sainte-Cécile à Loupian et villa de Milhaud (Foy, Michel, 2003). Les colorations et les analyses chimiques montrent clairement que diverses matières ont été utilisées, mais nous ne pouvons savoir si les vitres ont été importées ou réalisées dans les ateliers régionaux.

La vaisselle et le luminaire en verre verdâtre clair, souvent ornementés de filets de verre blanc opaque, rapportés en lignes horizontales ou en festons ou encore sous forme de bouton terminal, sont aujourd'hui identifiés sur de nombreux sites du midi de la France, mais aussi en Espagne (Sanchez de Prado, 1984, fig. 9 ; Marcos Pous, Vicent Zaragoza, 2000 ; Perez-Sala i Rodés, 2004). De plus, certaines coupes portent dans le fond un décor moulé chrétien. 


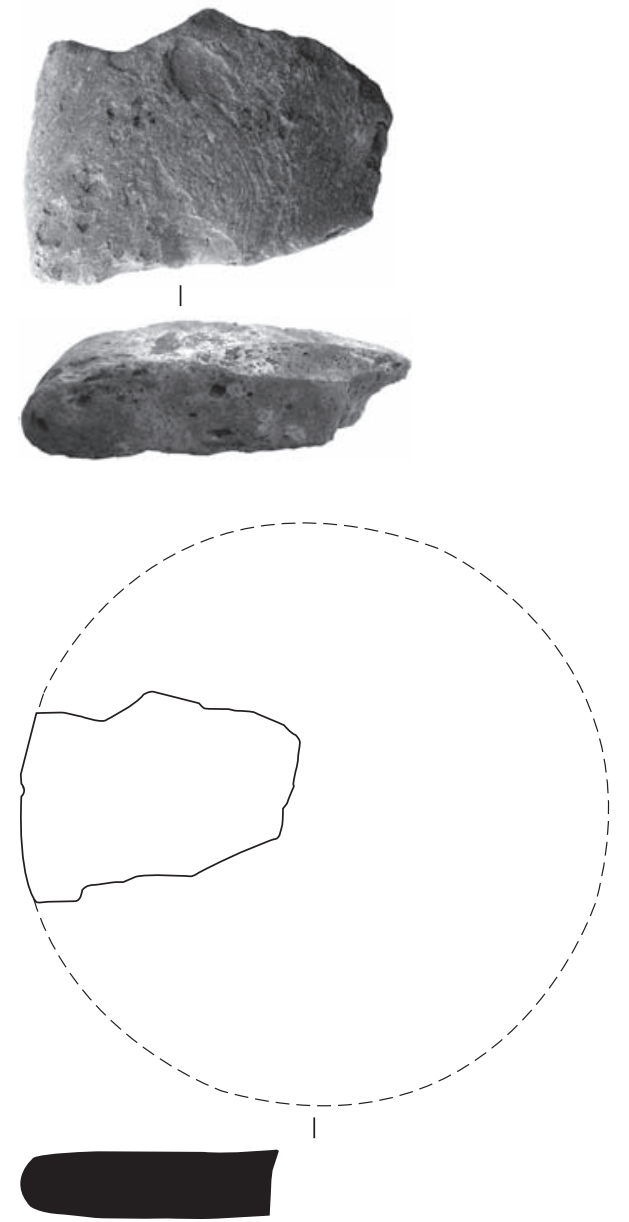

1
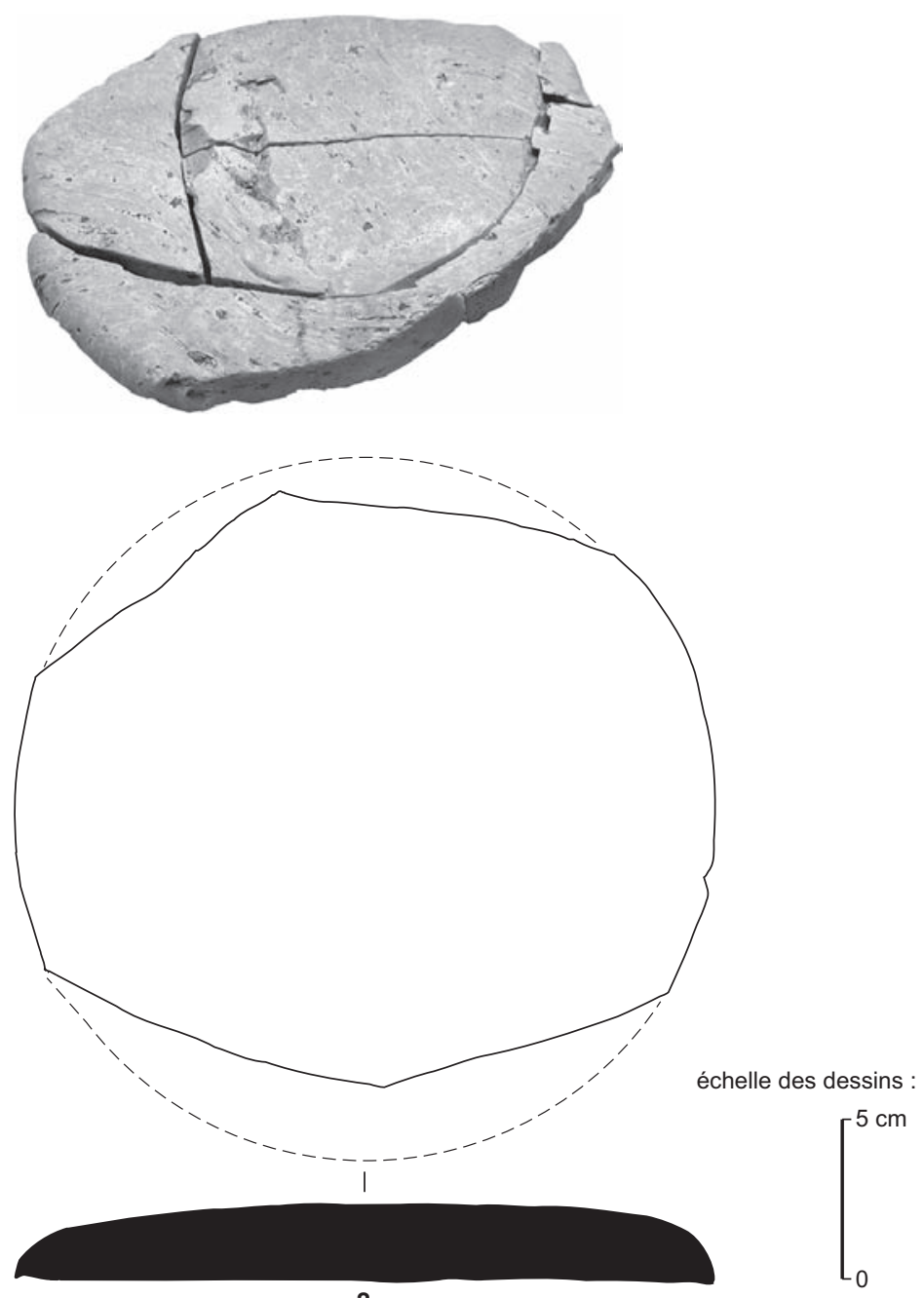

Fig. 76 - Production de verre : 1, galette de verre gris-bleu opaque, veiné de rouge, produit semi-fini pour tesselle, villa de Séviac, musée de Montréal, inv. 84 ; 2, galette de verre jaune opaque, produit semi-fini pour tesselles, Châteauneuf-du-Rhône (fouilles P. Carlier, 1983), musée d'archéologie tricastine, Saint-Paul-Trois-Châteaux (dessins et clichés : D. Foy, CNRS).

Fig. 77 - Production de verre : 1, coupe vert olive à décor abrasé, épave Port-Vendres I (fouilles : DRASSM), Ve s., importation égyptienne ?; 2, bouteille vert olive à décor abrasé, Narbonne, Hôtel-Dieu, début du $V^{e}$ s., importation égyptienne? ; 3, fiole soufflée dans un moule, Narbonne, Hôtel-Dieu, Ve s., importation égyptienne ?; 4, verre à pied incisé, verre brun, Marseille, la Bourse, Ve s., importation égyptienne ?; 5, fond de bouteille carrée avec inscription en grec, verre vert-jaune, Marseille, tunnel de la Major (fouilles : O. Maufras, INRAP), Ve-VI ${ }^{e}$., importation d'Asie Mineure; 6, coupe vert olive, Loupian, port du Bourbou (fouilles: C. Pellecuer), Ve s. ; 7, 8, gobelets vert olive à décor de pastilles bleues, épave Port-Vendres I, Ve s. ; 9, coupe à décor chrétien moulé, Toulouse, donjon du Capitole (fouilles : J. Catalo, INRAP), début $d u V^{e}$ s., longueur maximale: 7,2 cm; 10, coupe à décor de filets blancs rapportés, Marseille, la Bourse, début du VI $I^{e}$ s.; 11, fond d'un bol à

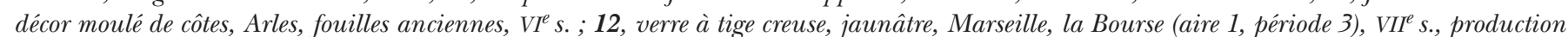
locale; 13, verre à tige creuse, jaunâtre, Marseille, place Jules-Verne (JV 5, us 325), seconde moitié du VI e., production locale; 14, verre à tige pleine, jaunâtre, Marseille, la Bourse (D II 15, période 4A), VI ${ }^{e}-V I I^{e} s$. ?, importation africaine; 15, verre à tige pleine, verdâtre clair, Marseille, la Bourse (aire 2, secteur 2, us 549), VII ${ }^{e}$. ?, importation; 16, verre à tige torsadée, bleutée, Perpignan/Ruscino, VIII ${ }^{e}$., production locale ou importation d'Espagne; 17, bol bleuté, Marseille, place Jules-Verne (JV 4, us 87), VII ${ }^{e}-V_{I I}{ }^{e}$ s. ? (n ${ }^{o s} 1,2,4-8,10-17$, dessins et clichés : D. Foy, CNRS ; $n^{\circ}$ 3, cliché : Y. Rigoir, LAMM; $n^{\circ}$ 9, cliché : C. Durand, CNRS). 

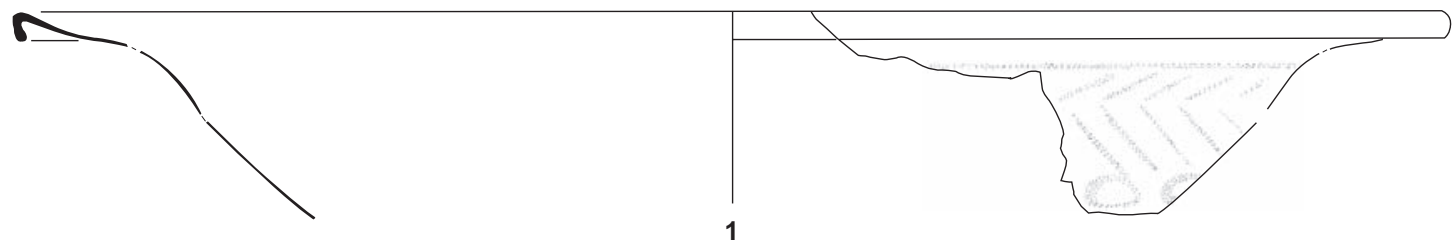

échelle des dessins
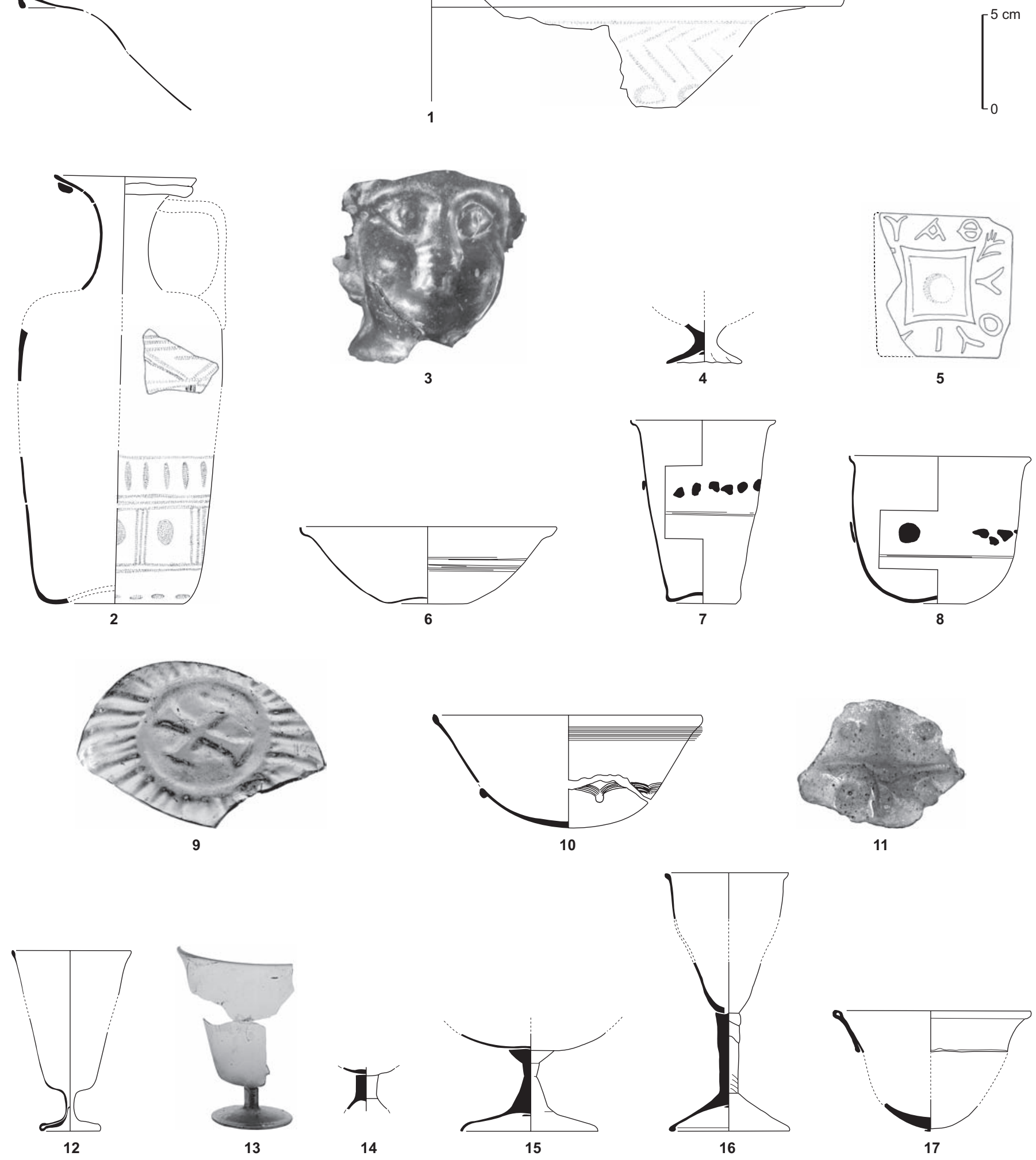
D'abord reconnue à Marseille dans les fouilles de la Bourse, cette catégorie de matériel présente dès la fin $\mathrm{du} \mathrm{V}^{\mathrm{e}} \mathrm{s}$. apparaît comme prédominante dans le premier tiers du VI ${ }^{\mathrm{e}}$ s. (fig. 77, $\mathrm{n}^{\mathrm{o}} 10$ ). Les fouilles d'Olbia (Fontaine, 2006, fig. 29 et 30), de Toulon, de nombreux secteurs de Marseille, de Saint-Blaise, de l'oppidum de Constantine, le dépotoir portuaire de Port-Vendres I mais aussi les habitats du sud-ouest de la France (Foy, Hochuli-Gysel, 1995, fig. 10 et 14 ; Catalo et al., 1998 ; Cornardeau, 2005) et de la région lyonnaise (Faure-Boucharlat, Vicherd, 2001) ont fourni un mobilier important. Le catalogue des pièces portant un décor chrétien moulé (croix flanquée ou non de croisettes ou autres emblèmes et chrismes, voir Foy, 1993) s'est aussi considérablement élargi. Une quarantaine de pièces, réparties entre Toulon, Notre-Dame-du-Bourg à Digne-les-Bains, Olbia, Gardanne, Marseille, l'oppidum de Constantine, Toulouse (fouilles du rectorat Saint-Jacques et donjon du Capitole) (fig. 77, $\mathrm{n}^{\mathrm{o}} 9$ ) et Bordeaux, sont à ce jour comptabilisées. Cette verrerie, qui présente de nombreuses parentés avec le mobilier des régions plus septentrionales (Cabart, Feyeux, 1995 ; Feyeux, 2003), a sans doute été soufflée dans les ateliers régionaux comme le laissent penser les indices recueillis à Gardanne (Pelletier et al., 1991, p. 337-338) et à Marseille (fouilles de la Bourse et du quartier Sainte-Barbe : Foy, 1997, p. 37 et 1998 p. 171173).

Les bols à décor moulé de côtes se rejoignant au centre $\mathrm{du}$ fond autour d'un motif cruciforme (Feyeux, 2003, forme 55), en usage dans la seconde moitié du VI ${ }^{\mathrm{e}} \mathrm{s}$. et au début du siècle suivant, sont extrêmement rares dans le sudest de la Gaule; nous n'en connaissons qu'un exemplaire anciennement trouvé à Arles (fig. 77, no 11). En revanche, cette forme bien documentée dans le nord de l'Europe est signalée en Aquitaine, à Bordeaux (Foy, Hochuli-Gysel, 1995, fig. 15, no 2), Chastel-sur-Murat (Cornardeau, 2005, fig. 4, $\mathrm{n}^{\mathrm{o}} 72$ ) et la Gravette à L'Isle-Jourdain (Cornardeau, 2004-2005, fig. 10,143).

Le verre à tige qui apparaît sans doute dans la première moitié du VI ${ }^{\mathrm{e}} \mathrm{s}$. devient, dans la seconde partie de ce siècle, le verre à boire le plus commun (fig. $77, \mathrm{n}^{\text {os }} 12$ et 13 ) ; il le restera pendant plus d'un siècle. Contrairement aux verreries à filets blancs qui sont communes à de nombreuses régions qui s'étendent des bords de la Méditerranée jusqu'au nord de la Gaule, cette nouvelle forme est principalement en usage dans les régions méditerranéennes et dans la vallée du Rhône (Faure-Boucharlat, Caclin, 2001). De part et d'autre de la Méditerranée, ce verre, utilisé pour boire mais peut-être aussi pour s'éclairer, présente de nombreuses variantes dont certaines peuvent être rattachées à des centres de fabrication. Le modèle le plus fréquent en Méditerranée occidentale se caractérise par sa coupe tronconique et sa tige évidée (fig. $77, \mathrm{n}^{\mathrm{os}} 12$ et 13) ; il est produit dans différentes officines dont celle de Maguelone en Languedoc (fig. 78) qui fabriquait aussi des lampes coniques et celle de Marseille (creusets et nombreux déchets de soufflage et de verre brut analysés : Foy, 1998, p. 132 ; Foy et al., 2003, p. 55, analyse d'un mors VRR 337). De rares importations peuvent être distinguées : les verres à tige pleine épaisse et courte (Foy, 1998, p. 131-132, nº 198 ; voir ici fig. 77, $\left.\mathrm{n}^{\mathrm{o}} 14\right)$, sont caractéristiques des ateliers africains (Foy, 2003, p. 73-75, type A) ; les tiges pleines, mais fines et resserrées en leur milieu (fig. 77, no 15) sont présentes en de nombreux lieux distants les uns des autres (Italie du Nord, mer Noire, Afrique, Égypte) sans que l'on puisse déterminer leur origine.

Vers le milieu du VII ${ }^{\mathrm{e}}$ s., se dessine un nouveau faciès de la verrerie qui fait en partie écho à ce que l'on connaît dans une grande partie de l'Europe. La teinte bleutée caractérise l'ensemble de ce mobilier qui comprend deux types de bols (dits palm cup) bien représentés dans le nord de la Gaule et les pays limitrophes (formes: Feyeux, 1995 et 2003, n ${ }^{\text {os }} 57$ et 60 ou Foy, 1995, $\mathrm{n}^{\text {os }} 28 \mathrm{a}$ et b). Les bols à fond arrondi et rebord formant un large ourlet rabattu à l'extérieur (fig. 77, $\left.n^{\circ} 17\right)$ sont signalés sur plusieurs sites, de Bordeaux jusqu'en Provence orientale. Ces verreries semblent encore en usage au IX ${ }^{\mathrm{e}}$ s., selon la datation fournie par les analyses de laboratoire du mobilier du prieuré Saint-Côme-et-Saint-Damien à La Cadière-d'Azur (Broecker, 2001). Des bols plus étroits à rebord ourlé à l'intérieur, parfois décorés de côtes ou de filets rapportés sous le bord, sont tout aussi présents même dans les habitats ruraux.

Les verres à tige pleine lisse ou torsadée semblent en revanche spécifiques des régions méditerranéennes, et peut-être plus précisément des terres wisigothiques. Ils ont été découverts à Perpignan et dans l'habitat languedocien de Dassargues dans des contextes qui ne peuvent être antérieurs au VIII ${ }^{\mathrm{e}}$ s. (Foy, 1995, forme 27 ; voir ici fig. 77, $\mathrm{n}^{\mathrm{o}} 16$ ). Nous ignorons si ces verres relèvent de productions locales ou bien d'importations de la péninsule Ibérique. Des pièces comparables sont connues en Espagne (Ortiz Palomar, 2001, fig. 131-132).

L'ensemble des indices de production (principalement déchets de soufflage et creusets vitrifiés) permet de recenser une vingtaine d'officines secondaires actives entre le $\mathrm{IV}^{\mathrm{e}}$ et le VIII ${ }^{\mathrm{e}} \mathrm{s}$. Les ateliers sont implantés aussi bien aux portes des grandes cités, à l'intérieur de villages parfois éloignés 


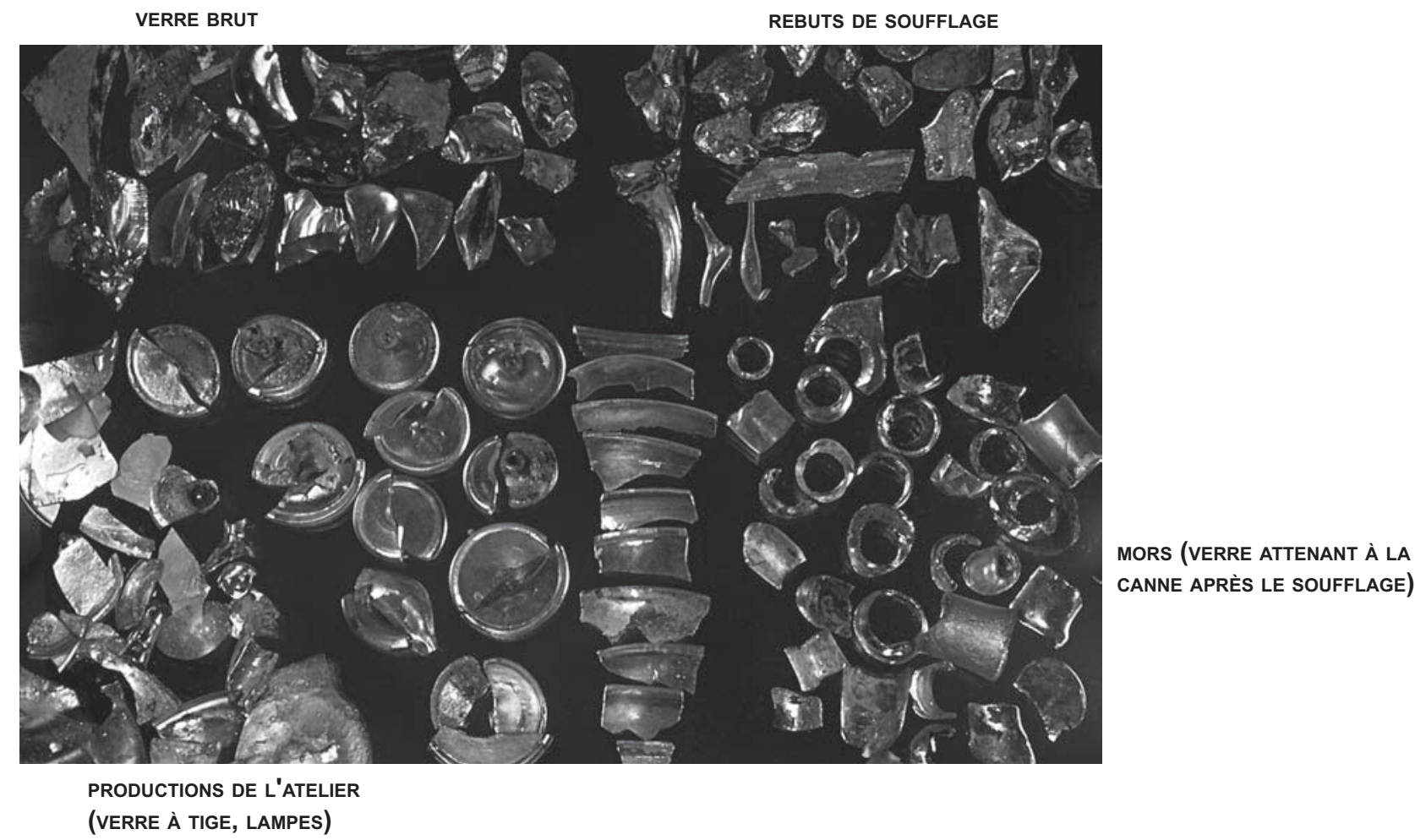

Fig. 78 - Atelier de verrier de Maguelone, fin $\mathrm{VI}^{e}-\mathrm{VII}^{e}$ s. (fouilles : J.-C. Richard) : en bas à droite, mors de verrier; en bas à droite et au centre, fragments de pieds et de rebords de verres à tige, produits localement (cliché : C. Durand, CNRS).

du littoral d'où arrivait le verre brut, ou encore dans l'orbite d'un centre épiscopal : Bordeaux, Perpignan, Maguelone, Roc de Pampelune à Argelliers (Schneider, 2003), Arles (fouilles des thermes, de l'Esplanade et rue Truchet), Eyguières, Méthamis, Saint-Mitre-les-Remparts, Marseille, Gardanne, Gémenos, Fréjus, Antibes, Viviers et Vienne (Foy, Nenna dir., 2001, p. 47-60). Nous sommes surtout renseignés sur l'artisanat marseillais dont les traces sont perceptibles en de nombreux points de la ville (la Bourse, tunnel de la Joliette, butte Saint-Laurent, quartier SainteBarbe, parking des Phocéens, Alcazar, parking Jules-Verne), indiquant sans doute l'existence de petites unités de fabrication dispersées dans la cité et surtout hors les murs, principalement au sud-est de la ville. Les fouilles de la Bourse, en particulier, permettent d'assurer que des ateliers ont été actifs durant cinq ou six générations, entre le deuxième quart du $\mathrm{V}^{\mathrm{e}}$ s. et la fin du VI ${ }^{\mathrm{e}}$ s. (Foy, 1998, p. 374-375).

Il est néanmoins rarement possible de connaître les produits qui sortaient de ces centres. Nous savons seulement que les coupes et les gobelets de teinte olivâtre ou jaune sombre étaient fabriqués à Marseille et sans doute aussi à Arles. Au début $\mathrm{du} \mathrm{VI}^{\mathrm{e}} \mathrm{s}$., nombreuses étaient sans doute les officines qui soufflaient la vaisselle et le luminaire décorés de filets de verre blanc appliqués et parfois de motifs chrétiens estampés sous le fond. Ces pièces sont uniformément répandues sur tout le territoire de la Gaule, mais peu d'ateliers sont formellement reconnus (peut-être Gardanne et Marseille). L'un des acquis de la recherche récente est d'avoir montré que ce type de verre caractérisé par son ornementation n'est pas absent des terres méridionales puisqu'on le trouve jusque dans le sud de l'Espagne (voir supra, p. 139).

Les verres à tige des $\mathrm{VI}^{\mathrm{e}}-\mathrm{VII}^{\mathrm{e}} \mathrm{s}$. sont des productions de Marseille et de l'atelier de Maguelone qui présente les témoins les plus éloquents (fig. 78). D’autres centres fabriquaient certainement les mêmes formes à partir de verre brut issu de quelques ateliers primaires de Méditerranée orientale. L'uniformité des matières et des formes explique la difficulté, voire l'impossibilité, de mettre en corrélation produits finis et centres de fabrication.

L'apport des études archéométriques a été fondamental pour déterminer les sources d'approvisionnement des artisans. Ces travaux de laboratoire ont contribué à la mise en évidence de la chaîne de production du verre antique dans laquelle les ateliers occidentaux apparaissent comme 
dépendants des centres primaires de Méditerranée orientale qui élaborent la matière première. À partir des analyses chimiques du verre brut et des produits finis, plusieurs groupes de composition utilisant les mêmes gisements de sable ont été identifiés (Foy et al., 2003). Ses résultats révèlent ainsi un commerce du verre brut important et permettent de distinguer trois temps dans l'histoire des techniques verrières occidentales.

- Pendant une longue période antique, les artisans dépendants des ateliers primaires orientaux refondent le verre brut importé. Celui-ci provient essentiellement - mais non exclusivement - des fabriques de la côte syro-palestinienne. Au tout début du $\mathrm{V}^{\mathrm{e}}$ s., il semble que l'Égypte ait réussi à ravir une part du marché destiné à la Méditerranée occidentale. Ces importations égyptiennes, certainement concurrencées par les produits syro-palestiniens dont l'arrivée n'a jamais cessé, occupent une place importante pendant le $\mathrm{V}^{\mathrm{e}} \mathrm{s}$. Dans la seconde moitié du VI ${ }^{\mathrm{e}} \mathrm{s}$. et durant une grande partie du siècle suivant, les officines du littoral méditerranéen occidental (rives septentrionale et méridionale) reçoivent du verre brut en provenance d'une même région encore non localisée. Vers la fin du VII ${ }^{\mathrm{e}}$ s., le verre brut de Palestine alimente encore de nombreux ateliers orientaux et quelques centres occidentaux, en particulier des officines africaines. En revanche, peu d'apports sont perceptibles sur les côtes septentrionales.

- Les pratiques de refusion du verre, déjà perceptibles dans les productions du $\mathrm{V}^{\mathrm{e}} \mathrm{s}$., s'amplifient à partir de la fin du VII ${ }^{\mathrm{e}} \mathrm{s}$. Elles pourraient traduire la volonté de s'affranchir de la dépendance des ateliers primaires extérieurs, mais elles peuvent aussi signifier, sinon un arrêt total des apports, du moins une réduction de la circulation des marchandises en Méditerranée qui oblige les artisans à travailler en autarcie en recyclant le verre.

- C'est probablement vers la fin du IX ${ }^{\mathrm{e}} \mathrm{s}$. qu'apparaît un changement radical dans la fabrication du verre. L'adoption de nouvelles recettes, sur les deux rives de la Méditerranée (utilisation de cendres végétales locales), rend les ateliers occidentaux indépendants.

\section{F. LE MOBILIER MÉTALLIQUE}

\section{LES AUTRES PRODUCTIONS}

\section{LA PIERRE OLLAIRE}

Plusieurs roches métamorphiques d'origine alpine, généralement de couleur verte, ont été utilisées dans l'Antiquité pour la fabrication de récipients, ce qui leur a valu l'appellation générique de pierre ollaire. Précocement et particulièrement attestées dans les régions alpines, du Jura au Piémont, ces productions connaissent une plus ample diffusion à la fin de l'Antiquité où on les trouve tout au long de l'axe Saône-Rhône, atteignant le Massif central ainsi que la vallée du Rhin (Billoin, 2003). Bien attestés dans la région Rhône-Alpes du $\mathrm{III}^{\mathrm{e}}$ au VIII ${ }^{\mathrm{e}} \mathrm{s}$. (Lhemon, 2003), les récipients en pierre ollaire atteignent plus tardivement le rivage méditerranéen où ils sont principalement représentés à la fin $\mathrm{du} \mathrm{V}^{\mathrm{e}} \mathrm{s}$. et au $\mathrm{VI}^{\mathrm{e}} \mathrm{s}$., ainsi que l'ont montré de nombreuses fouilles d'habitat, notamment à Saint-Blaise où a été recueillie la série la plus conséquente du Midi méditerranéen (Démians d'Archimbaud dir., 1994, p. 198-200). Quant à la diffusion vers le Sud-Ouest, elle reste méconnue en l'état des recherches.

Réalisés par tournage dont les parois conservent de nombreuses cannelures, les récipients affectent pour la plupart des profils cylindro-coniques à fond plat et à bord droit à lèvre arrondie (fig. 79). Sans être abondants, partout les vases en pierre ollaire entrent dans le vaisselier domestique, à la ville comme à la campagne où ils prennent place parmi le mobilier culinaire, ainsi qu'en témoignent de fréquentes traces de feu ayant altéré la roche, ou encore des dépôts de suie observés sur leurs parois. Certains exemplaires découverts dans des ateliers métallurgiques posent par ailleurs la question de leur utilisation comme creusets, qui a été envisagée mais reste à démontrer. Plus anecdotique apparaît la réalisation de fusaïoles comme celle découverte dans une sépulture du VI ${ }^{\mathrm{e}} \mathrm{s}$. à Lunel-Viel (Raynaud dir., à paraître). Si ces récipients apparaissent de façon récurrente dans les contextes tardo-antiques, ces éléments demeurent cependant de médiocres indicateurs chronologiques dans la mesure où leur typologie ne laisse apparaître aucune évolution autorisant un découpage fin. Cette production ne semble pas attestée par la suite, les contextes du haut Moyen Âge n'en ayant livré aucune attestation.

C. R.

Depuis la parution en 1977 de la synthèse d'E. James, la connaissance du mobilier de parure de l'Antiquité tardive a connu de sensibles avancées. Depuis une décennie, cette avancée est redevable au développement des fouilles de nécropoles, singulièrement à L'Isle-Jourdain (Bach, Boudartchouk, 1998), Lunel-Viel et Maguelone (Raynaud dir., à paraître). Les fouilles d'habitat livrent aussi leur contribution, moins sensible toutefois que dans les contextes 

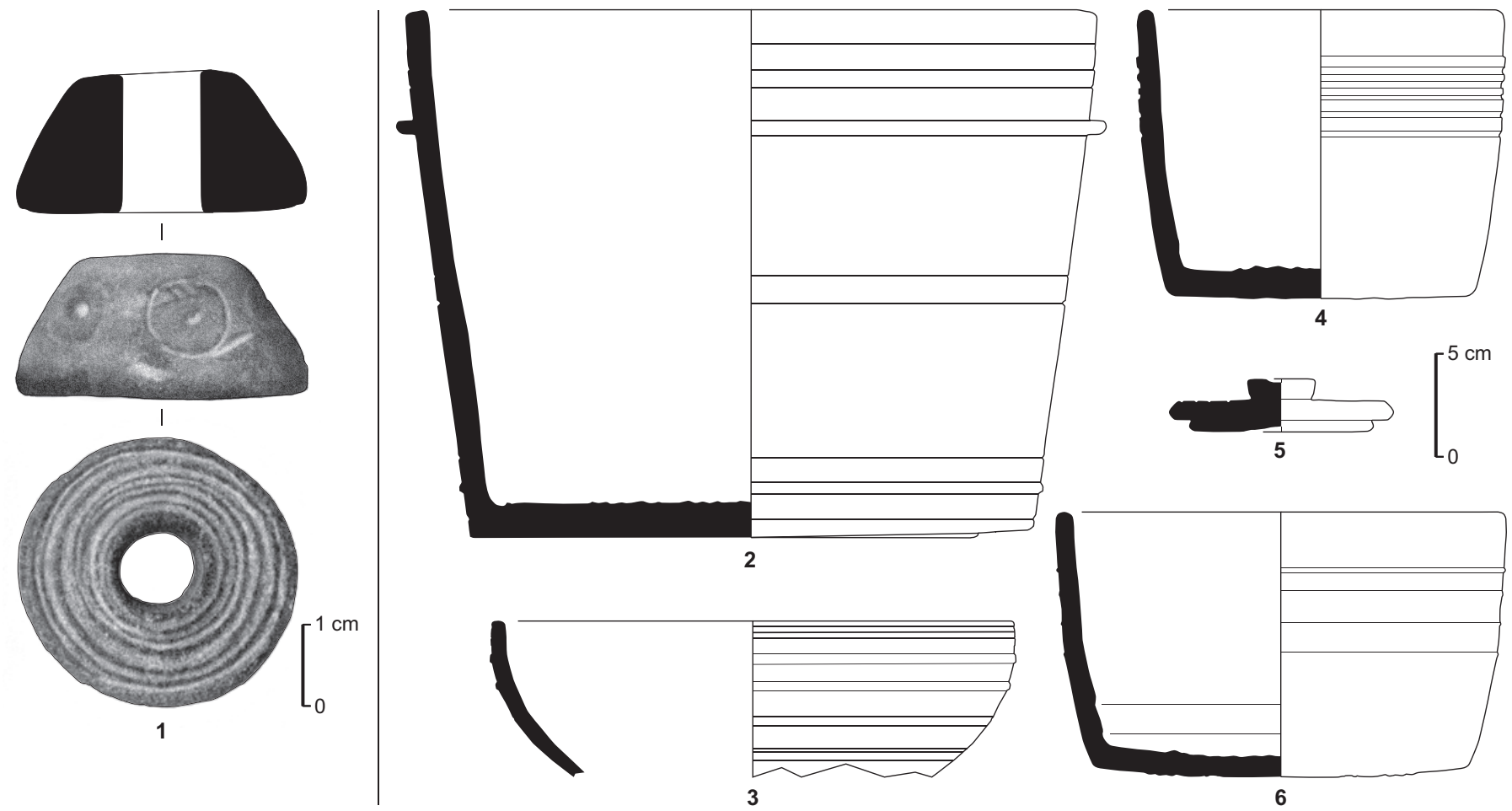

Fig. 79 - Production en pierre ollaire : Lunel-Viel, nécropole des Horts (Raynaud dir., à paraître, pl. 4) ; région Rhônes-Alpes (Lhemon, 2003, $n^{o s} 1$-5) ; oppidum de Saint-Blaise (Démians d'Archimbaud dir., 1994, nos 8-9) (DAO : M. Ott, INRAP).

funéraires. Cet enrichissement documentaire, doublant parfois le volume des connaissances antérieures, a suscité dans un second temps la réalisation de recherches systématiques reposant sur d'amples corpus. Une première approche a permis de mesurer la permanence, aux $\mathrm{IV}^{\mathrm{e}}$ et $\mathrm{V}^{\mathrm{e}} \mathrm{s}$., d'un fonds commun à l'ensemble des provinces occidentales (Feugère, 1993). L'évolution est plus sensible dès la fin du $\mathrm{V}^{\mathrm{e}} \mathrm{s}$. avec la diffusion d'un mobilier original au sein duquel se mêlent influences wisigothiques, septentrionales (Stutz, 1996), et plus tard byzantines. Si nombre d'objets composent un fonds d'usage courant dans l'ensemble de l'Europe occidentale, les assemblages permettent de définir plusieurs faciès, en Aquitaine (Lerenter-Jilet, 1991), Toulousain, Septimanie (Hernandez, 2001) ou Provence (Stutz, 2005). Pour autant, ces ensembles régionaux demeurent rétifs à une cartographie précise, les assemblages observés tant dans les tombes que dans l'habitat faisant apparaître de nombreuses nuances qui interdisent d'envisager les contextes régionaux comme autant d'entités à bords francs. Partie d'une première approche à visée ethniciste, la recherche incline donc de plus en plus nettement vers une approche en termes de changement culturel et de métissage, à l'instar des nécro- poles de Septimanie où il est bien délicat de distinguer ce qui, des influences septentrionales, wisigothiques, byzantines et du fonds régional, domine la mode vestimentaire (Hernandez, Raynaud 2005). Dans cette région en effet, la domination wisigothique durant près de deux siècles ne se traduit guère par une influence manifeste sur les modes vestimentaires. Pour autant, la région s'avère très ouverte aux influences exogènes, particulièrement sensibles à travers les pièces de mobilier d'origine septentrionale, deux à trois fois plus nombreuses que les éléments de faciès wisigothique. Dans les habitats et les nécropoles récemment fouillés, à Lunel-Viel comme à Maguelone, l'association d'objets de provenances diverses témoigne de contacts fréquents et d'un intense brassage culturel. Tout en invitant à nuancer l'impact effectif de la culture wisigothique en Septimanie, le mobilier de parure confirme les observations fondées sur les céramiques, révélant l'intensité des échanges nord-sud. Ainsi faiblit l'idée ancienne d'une frontière étanche avec le monde franc (James, 1980) : plus qu'une ligne de démarcation, ce sont des franges perméables qu'il faut désormais retenir entre les royaumes romano-germaniques.

C. R. 


\section{CONCLUSION}

Voilà donc posés les éléments d'un cadre typochronologique pour la Gaule du Sud-Est et les régions voisines. Éléments foisonnants mais encore disparates dans leur répartition géographique, éléments affinés mais parfois incertains, ces jalons donnent à l'historien une prise sur la vie quotidienne et les échanges, sur les techniques aussi, quoique beaucoup reste à faire pour localiser les ateliers et caractériser leurs processus de production et de diffusion. Immanquablement, l'avancement des travaux pose des questions jusqu'alors hors d'atteinte, et ouvre des chemins peu frayés à ce jour. Dans cet échafaudage encore partiel, l'interprétation des monnaies demeure délicate en raison, d'une part, de leur relative rareté dans les fouilles récentes et, d'autre part, de la pratique du surmoulage qui rend difficile la détermination des phases d'émission. Il en est de même des objets de parure dont on voit progresser trop faiblement la datation. Enfin, si l'on a vu s'améliorer de manière spectaculaire la fiabilité de la chronologie de la vaisselle de verre, il reste que les données céramologiques occupent une fonction décisive et souvent exclusive, au point que l'on peut craindre la circularité des datations : des céramiques datées par des céramiques qui sont datées par des céramiques, en abyme... Pour échapper à cette boucle, il conviendrait d'aborder la chronologie sous l'angle des datations absolues, en favorisant les analyses ${ }^{14} \mathrm{C}$ qui, malheureusement pour l'heure, offrent une précision bien relative, souvent inférieure à celle d'une «bonne vieille » typologie céramique. Doit-on le regretter? Non si l'on fouille dans une région littorale - et moins encore si l'on est dans un port - où affluent les importations et où les possibilités de comparaisons se multiplient, mais c'est par contre dommageable aux recherches conduites dans les arrière-pays où les produits méditerranéens ne parviennent pas ou peu. D’où la nécessité de poursuivre l'accumulation documentaire qui, sans jamais devenir une fin en soi, doit demeurer le fond de la démarche archéologique, particulièrement pour ce qui concerne les productions régionales, plus largement diffusées à l'intérieur des terres.

Si la constitution et l'édition des corpus documentaires demeurent une préoccupation constante, parce que la discipline revient de loin, de très loin dans la vacuité, les premiers éléments autorisent à s'interroger sur les modes de diffusion et de transfert, aussi bien des poteries que des habitudes alimentaires qu'elles dénotent: de Palestine en Tunisie, de Tunisie en Provence, de Provence en Bourgogne et retour, la circulation demeure intense jusqu'à un $\mathrm{VI}^{\mathrm{e}} \mathrm{s}$. avancé, avant d'entrer en décrue. Qu'échange-t-on, dans quels termes, à quelles échelles ? Chaque production, de la plus modeste production «commune" (mais ce n'est pas un critère de qualité, nous l'avons assez dit) jusqu'aux plats africains imitant la vaisselle de métal, contribue à façonner une ambiance culturelle qui à la fois affirme son ampleur mais en même temps laisse émerger de forts régionalismes. Nous sommes pourtant à cent lieux de la fragmentation attendue, aux antipodes de l'émiettement annoncé après la désagrégation du régime impérial. Est-ce une surprise? Certainement pas si l'on veut bien se défaire de la vision empesée d'un Empire unifié aux modes de vie stéréotypés qui n'a jamais existé que dans la rusticité des schémas historiques.

En vingt ans, la datation et la détermination de l'origine des différentes céramiques méditerranéennes importées, amphores, vaisselle fine et vaisselle commune, ont considérablement gagné en précision, bénéficiant du développement des recherches sur les lieux mêmes de production. Il est à souligner que certaines de ces recherches ont eu pour point de départ des questions posées en Provence et en Languedoc au milieu des années 1980 (Février, Leyge dir., 1986), qu'il s'agisse des productions africaines (Bonifay, 2004) ou des amphores et des céramiques culinaires orientales (Pieri, 2005 ; Tréglia, 2005).

Toutes proportions gardées, ces progrès sont comparables à ceux effectués dans les années 1960 et 1970, au moment où les «sigillées Claires » A-C-D de N. Lamboglia ont été reconnues comme africaines et leur datation, initialement limitée au V ${ }^{\mathrm{e}}$ s., étendue jusqu'au VII ${ }^{\mathrm{e}} \mathrm{s}$. Ainsi, alors que les «sigillées africaines " étaient comprises de façon globale en 1986, il est aujourd'hui possible de reconnaître les productions du nord et du centre de la Tunisie, voire même les produits de quelques ateliers spécifiques: El Mahrine, Oudhna ou Sidi Khalifa. Dans le domaine des productions orientales, on sait distinguer des amphores d'Égypte, de Syrie-Palestine, de Cilicie, de Chypre, même lorsque les formes sont semblables et, avec un peu d'expérience, on peut identifier des marmites provenant du Levant, de Lycie ou de Constantinople. De même, la multiplication des publications de contextes stratigraphiques de référence en Méditerranée nord-occidentale (Tarragone, Rome, Naples), en Afrique (Carthage) et en Méditerranée orientale (Alexandrie, Istanbul) permet de consolider les chronologies de Gaule du Sud, établies de manière encore très hésitante en 1986. On a gagné en précision, notamment à l'intérieur $\mathrm{du} \mathrm{V}^{\mathrm{e}} \mathrm{s}$., et en durée, jusqu'à atteindre sans grand risque d'erreur les dernières décennies du $\mathrm{VII}^{\mathrm{e}} \mathrm{s}$. ou le début du VIII ${ }^{\mathrm{e}} \mathrm{s}$. 
Ces avancées, en partie redevables à l'engagement des archéologues français dans les missions à l'étranger après deux décennies de repli (postcolonial ?) sur l'archéologie métropolitaine, montrent combien, jusqu'à la fin de l'époque mérovingienne, le sud de la Gaule reste soumis aux pulsations de l'activité économique et des conditions politiques du Bassin méditerranéen. Qu'il s'agisse des flux d'amphores orientales au $\mathrm{V}^{\mathrm{e}} \mathrm{s}$., de la diminution des importations de sigillées africaines dans la seconde moitié du $\mathrm{V}^{\mathrm{e}}$ s., de la part encore importante de l'Afrique dans les approvisionnements de denrées aux $\mathrm{VI}^{\mathrm{e}} \mathrm{s}$. et $\mathrm{VII}^{\mathrm{e}} \mathrm{s}$., toutes ces observations faites en Gaule du Sud trouvent un écho dans d'autres parties du Bassin méditerranéen. Il n'est pas jusqu'au renouveau des productions de vaisselle fine en Provence dans la seconde moitié $d u V^{e}$ s. qui ne puisse être comparé au développement des productions de sigillées phocéennes, chypriotes ou égyptiennes à la même époque. Et ce ne sont pas seulement les villes du littoral qui réagissent à l'unisson des autres grands ports de Méditerranée ; il en est ainsi également des villes reliées à la mer par un fleuve navigable - à l'exemple de Lyon - dont la consommation céramique, sur bien des points, répond à celles de Saragosse, des villes de la vallée du Pô ou même de la HauteÉgypte. Enfin, il est remarquable, de façon peut-être plus originale, que bien des sites ruraux du sud de la Gaule, villae de plaine autant qu'habitats de hauteur, demeurent dans ce réseau jusqu'au milieu du $\mathrm{VI}^{\mathrm{e}} \mathrm{s}$. et même dans certains cas jusqu'au début du VIII' s., à l'image de San Peyre à Suzon, au pied des Cévennes.

Il reste maintenant, par des études régionales plus systématiques et n'hésitant pas à réviser certaines études anciennes, à mieux cerner, dans ces grandes tendances, la part des conditions macro-économiques et celle des conditions locales du marché et de la production. Cette démarche comporte toutefois un point faible : notre mauvaise connaissance du contenu de nombreuses amphores. Quelles étaient les denrées importées dans les amphores africaines et dans certains types d'amphores orientales (par exemple le type $L R A$ 2) des $\mathrm{V}^{\mathrm{e}}, \mathrm{VI}^{\mathrm{e}}$ et $\mathrm{VII}^{\mathrm{e}} \mathrm{s}$. ? C'est sans doute, pour mieux comprendre l'économie régionale de l'Antiquité tardive, l'un des points essentiels sur lesquels doivent porter nos efforts dans les années à venir.

Esquissée à grands traits, la géographie des productions de la Gaule méridionale à la fin de l'Antiquité fait apparaître de nombreuses lacunes mais encourage aussi à poursuivre l'enquête, de nombreux dossiers restant à étudier dès que l'on s'éloigne des rivages méditerranéens et de la vallée du Rhône (fig. 80). Bien évidemment, les premiers éléments

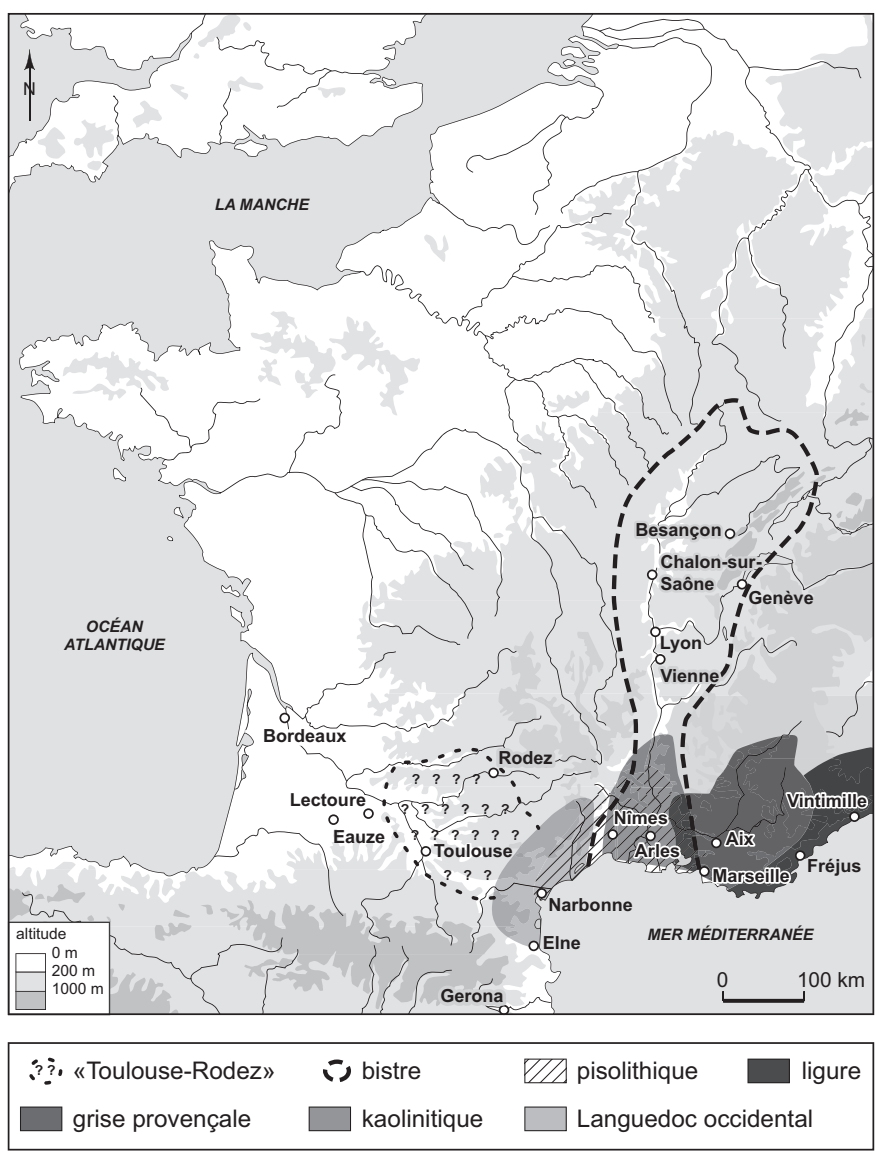

Fig. 80 - Carte de diffusion des principales productions de céramique commune, du $V^{e}$ s. au VI $I^{e}$. (dessin : C. Raynaud, CNRS; DAO : M. Ott, INRAP).

manquent de sûreté, notamment en ce qui concerne la cartographie des aires de diffusion. Il reste à réaliser une enquête systématique dans les collections, ainsi qu'un cortège d'analyses de laboratoire seules à même de préciser les aires de production et les identifications.

De la fin $\mathrm{du} \mathrm{IV}^{\mathrm{e}} \mathrm{s}$. au VI ${ }^{\mathrm{e}} \mathrm{s}$, la production à pisolithes du Bas-Languedoc, notre point de départ, se trouve enchâssée dans un maillage de productions que l'on peut qualifier de « régionales». Le terme doit être compris dans le sens où des ateliers ou des groupes d'ateliers diffusent leurs produits à des distances comprises entre $100 \mathrm{~km}$ et $200 \mathrm{~km}$, voire plus pour certaines, sortant du cadre local. Voilà un trait marquant qui émerge des études récentes, comme le montre l'évolution de la distribution dans la région liguroprovençale entre le Haut- et le Bas-Empire. C'est une piste encore cahotante, car la cartographie des faciès demeure très lacunaire, mais voilà une idée à creuser autour d'une possible mutation socio-économique du monde artisanal, 
qui verrait sa production échapper aux petites structures éclatées des $\mathrm{I}^{\mathrm{er}}$-III ${ }^{\mathrm{e}} \mathrm{s}$. et passerait à de grands ateliers à large diffusion. Conjecture certes, bien téméraire encore, mais nage-t-on sans se jeter à l'eau ?

Cette dimension régionale va (irait?) de pair avec de nombreuses parentés, tant dans le répertoire des formes que dans le détail de la typologie, ou encore dans le choix de privilégier presque systématiquement les argiles kaolinitiques. Le choix des techniques de cuisson entre aussi dans cette ambiance générale avec l'abandon progressif de la diversité alliant cuisson oxydante et cuisson réductrice, au profit de la monotonie qui voit s'imposer la cuisson réductrice donnant à la vaisselle sa grisaille, tôt en Provence et dans la Drôme où le basculement est opéré dès le $\mathrm{V}^{\mathrm{e}} \mathrm{s}$., plus tard en Septimanie où l'évolution semble aboutir seulement au début $d u \mathrm{VI}^{\mathrm{e}} \mathrm{s}$. Dans cette ambiance, la tradition galloromaine domine jusqu'au milieu du $\mathrm{VI}^{\mathrm{e}} \mathrm{s}$., dans la production méridionale comme dans la production saônoise, cette dernière demeurant étonnamment fidèle à la cuisson oxydante jusqu'au VIII ${ }^{\mathrm{e}}$ s., de façon tout à fait exceptionnelle. Ces importations saônoises, loin d'introduire une note exotique, ne font qu'étoffer la tradition antique dans laquelle toutes ces productions puisent l'essentiel de leur répertoire. La seule innovation introduite par la vaisselle du Val de Saône réside dans la récurrence des décors à la molette, absents jusqu'alors dans le Midi, et qui ne semblent pas introduits dans la production locale avant le VII ${ }^{\mathrm{e}} \mathrm{s}$. voire le VIII ${ }^{\mathrm{e}}$ s., bien après la disparition de la vaisselle à pisolithes. Lorsque les poteries kaolinitiques, désormais hégémoniques, adoptent cette technique, c'est pour orner l'épaulement de pots globulaires, après l'arrêt de la production des formes antiquisantes, bols, coupes, cruches et mortiers. La filiation avec la vaisselle bourguignonne est donc loin d'être établie. Les données font défaut pour cerner ce tournant « carolingien ».

Un autre mouvement se dessine dans le même temps, celui d'un basculement de l'aire d'approvisionnement en produits lointains. Longtemps restreintes à quelques unités, une céramique d'Argonne à Marseille et deux seulement à Lyon (Bonifay et al. dir., 1998, fig. 316 ; Ayala, 1998, p. 230), quelques pièces de céramique métallescente à Arles (Piton, 1998), à Loupian (Rouquette, Pellecuer, 1976-1977) ou Rodez (Boudartchouk, Llech, 1993, p. 154), les importations septentrionales en Gaule méditerranéenne croissent à mesure que faiblissent les flux maritimes. Sans atteindre le volume ni la diversité des céramiques méditerranéennes qu'ils n'ont jamais remplacés au demeurant, les arrivages croissent de façon sensible dès la fin $\mathrm{du} \mathrm{VI}^{\mathrm{e}} \mathrm{s}$., avec pour seul objet actuellement identifié les productions à pâte bistre de la vallée de la Saône, dont la diffusion reste à préciser mais ne fait aucun doute jusqu'au littoral. Verra-t-on là un signe, tout au moins un indice, en faveur de la vieille thèse de Pirenne sur le déplacement du centre de gravité de l'économie, de la Méditerranée vers l'Europe occidentale ? Malgré leur accroissement, les données demeurent lacunaires et leur cartographie encore aléatoire pour que l'on tranche ce débat: du moins ces questions en élargissent-elles le cadre.

Cette première cartographie des importations autant que des productions régionales répond ainsi à l'une de nos questions de départ, à propos de la fragmentation ethnique de la culture matérielle. Sans surprise s'impose l'image d'une continuité de la culture matérielle, peu troublée par la nouvelle donne politique. Si Burgondes, Wisigoths et Francs dominent la nouvelle géographie politique, les cadres de vie n'enregistrent aucun bouleversement jusqu'au début $\mathrm{du} \mathrm{VII}^{\mathrm{e}} \mathrm{s}$. au moins. Les changements s'opèrent plus tard et très progressivement, à une date encore mal cernée au VII ${ }^{\mathrm{e}}$ ou au VIII ${ }^{\mathrm{e}} \mathrm{s}$. En cela, la céramologie confirme les observations effectuées sur les réseaux de pleuplement, les formes de l'habitat et les techniques de construction. En regard de ces glissements progressifs, seulement perceptibles à l'échelle séculaire, combien formel et rigide apparaît désormais le concept de «haut Moyen Âge ».

M. B. et C. R. 


\section{NOTICES}

\section{NOTICE 1}

\section{AMPHORES DE MÉDITERRANÉE ORIENTALE DURANT L'ANTIQUITÉ TARDIVE (IVe-VII' S.) : RÉGIONS DE PRODUCTION ET COMMERCE VERS LA GAULE}

Nos connaissances acquises sur les amphores ces vingt dernières années ont permis de renouveler entièrement la vision historique mais surtout économique de l'Antiquité tardive et du haut Moyen Âge en Méditerranée. C'est notamment grâce aux études réalisées sur les matériels originaires du bassin oriental de la Méditerranée que les progrès en matière d'évaluation des échanges sont assez remarquables.

Les avancées les plus notables concernent deux domaines en particulier : d'une part, la mise en place de typologies qui permettent de suivre précisément l'évolution chronologique des différentes productions et d'évaluer ainsi les flux commerciaux ; d'autre part, la localisation des régions de fabrication qui permet de mieux connaître l'organisation générale de la production agricole associée aux mécanismes de distribution et d'approvisionnement.

Le phénomène le plus prégnant, mis en évidence dès 1987 lors d'une enquête menée en France (Bonifay, Villedieu, 1989), consiste en l'abondance, voire la suprématie, dès le début du $\mathrm{V}^{\mathrm{e}} \mathrm{s}$., des amphores originaires de Méditerranée orientale, omniprésentes sur tous les grands sites de consommation occidentaux : Rome, Naples, Narbonne, Marseille, Tarragone, Carthage. Dans le sud de la France, l'arrivée spectaculaire des importations orientales se traduit dans les statistiques par des chiffres très élevés durant une grande partie du $\mathrm{V}^{\mathrm{e}} \mathrm{s}$., atteignant en moyenne $30 \%$ à $45 \%$ du total des amphores recueillies dans les stratigraphies narbonnaises, arlésiennes et marseillaises.

Depuis, le corpus n'a cessé de s'enrichir révélant une diversité exceptionnelle des centres de production et un dynamisme important des régions orientales impliquées dans le grand commerce (Pieri, 1998 et 2005).

La destructuration des centres producteurs occidentaux depuis le milieu du III $^{\mathrm{e}}$ s., marquée par la disparition progressive des corporations de marchands romains et par le déclin des ateliers d'amphores ibériques et gaulois, ainsi que l'attrait commercial exceptionnel qu'exerce Constantinople à partir du milieu du $\mathrm{IV}^{\mathrm{e}} \mathrm{s}$. sur les provinces orientales, occasionne l'émergence de nouvelles régions productives jusqu'alors peu actives. Certaines régions se distinguent même par leur production spécialisée de denrées destinées à l'exportation. Tel est le cas de la Grèce continentale (Péloponnèse), de certaines îles de la mer Égée (Samos, Chios, Thasos), de la Crète, de Chypre, des franges occidentales et méridionales de l'Asie Mineure, des côtes méridionales de la mer Noire, du Levant et de l'Égypte.

Depuis les premières tentatives entreprises dans le dessein de définir des classements typologiques (Riley, 1982), notre connaissance du matériel oriental a considérablement évolué, mettant en valeur une production de conteneurs généralement circonscrite aux grands centres urbains orientaux mais également complétée par une multitude d'ateliers secondaires disséminés sur des aires géographiques vastes (Jal'ad, Galilée, Néguev, Maréotide, moyenne vallée du Nil). Les sept types originellement définis par Riley à Carthage (LRA 1-7) se sont vu complétés par bon nombre de variantes permettant de mieux suivre les évolutions chronologiques de chaque amphore (fig. 81). De plus, de nouvelles formes viennent régulièrement compléter le classement général en fonction des découvertes réalisées sur les sites consommateurs ou producteurs (Apamée, Beyrouth, Sinope, Aqaba). Une des nouveautés apportée par les études typologiques porte sur la reconnaissance d'amphores possédant des capacités standard déclinées en modules de plus petite contenance, mettant ainsi en évidence un système métrologique relativement complexe pour l'époque tardive.

Le vin oriental semble être le principal produit transporté dans ces amphores, connues des spécialistes sous le nom de Late Roman Amphoras (LRA). Les vins orientaux connaissent alors une grande notoriété, largement célébrée en Occident par des chantres aussi illustres que Sidoine Apollinaire, saint Jérôme et Grégoire de Tours ou Isidore de Séville.

À côté du commerce oriental très lucratif de l'ivoire, des pierres précieuses, des parfums, des papyrus, des épices et des étoffes, le vin semble donc être une des principales richesses de la pars orientalis. L'Orient a produit du vin partout, en grande quantité et d'excellente qualité mais aussi souvent très onéreux (fig. 82). Les auteurs anciens nous parlent des crus fameux de tout le Bassin méditerranéen 
LATE ROMAN AMPHORA 1

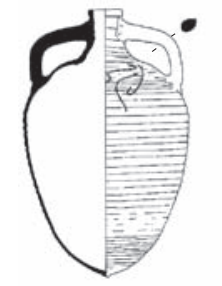

LRA 1A (fin IV $\left.-V^{e} s.\right)$

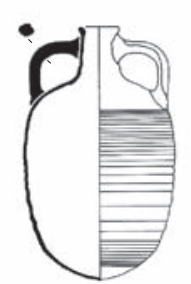

LRA 1A transition (fin $\mathrm{V}^{\mathrm{e}}$ - début $\mathrm{Vl}^{\mathrm{e}} \mathrm{s}$.)

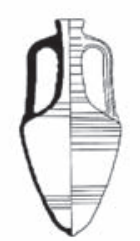

LRA 1A

sous-module
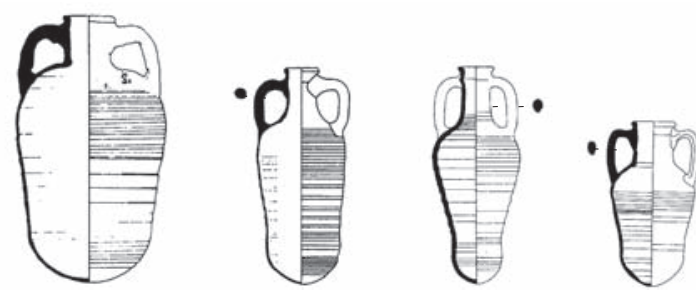

LATE ROMAN AMPHORA 2
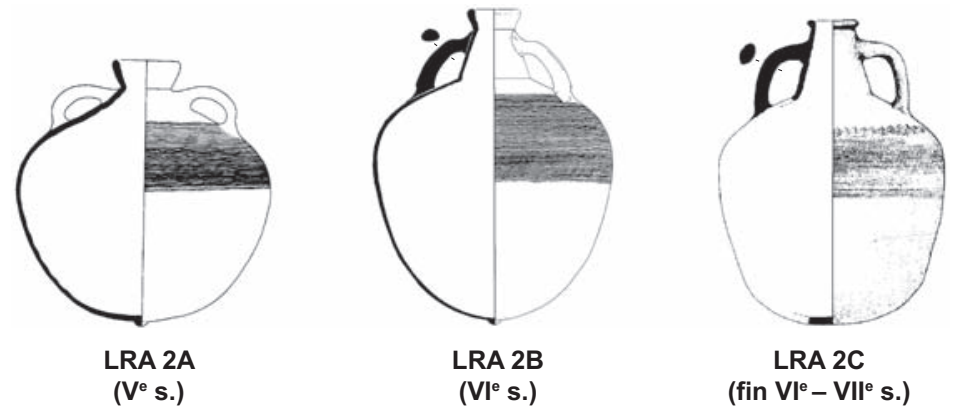

LATE ROMAN AMPHORA 3

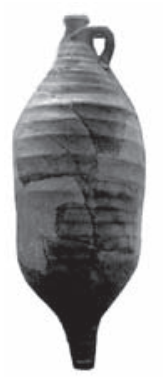

A1

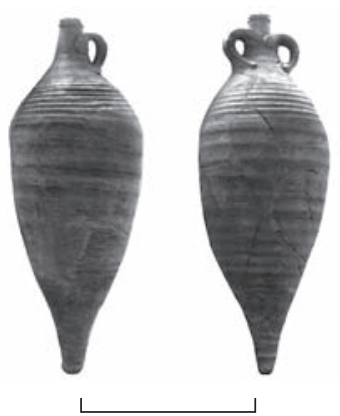

A2

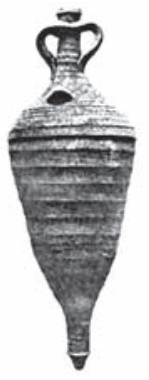

A3

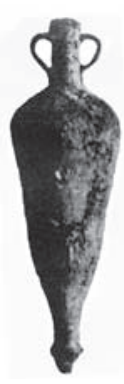

A4

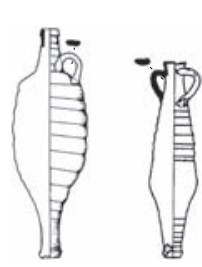

B1 B2
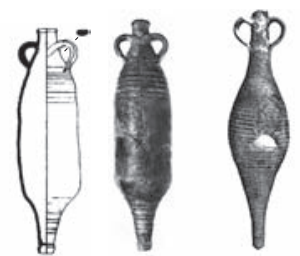

B4

LATE ROMAN AMPHORA 4

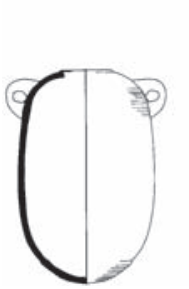

A1

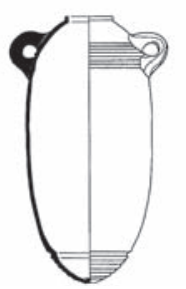

A2

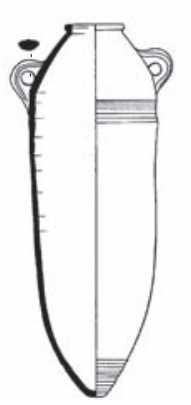

B1

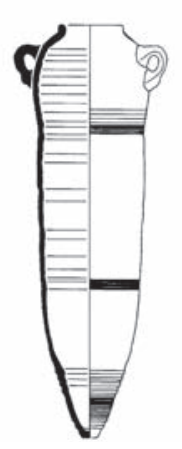

B2

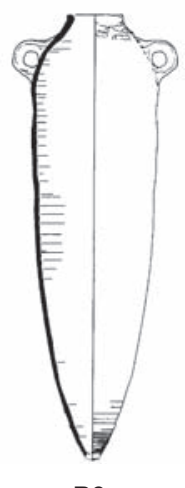

BAG-SHAPED AMPHORA

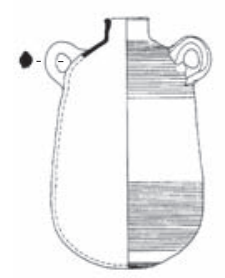

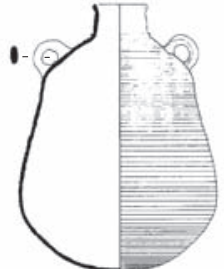

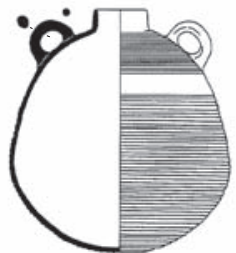

3

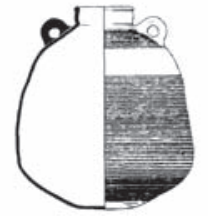

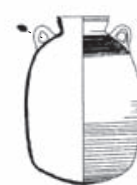

Fig. 81 - Typologie des amphores orientales (DAO : D. Pieri, Université Paris-I). 


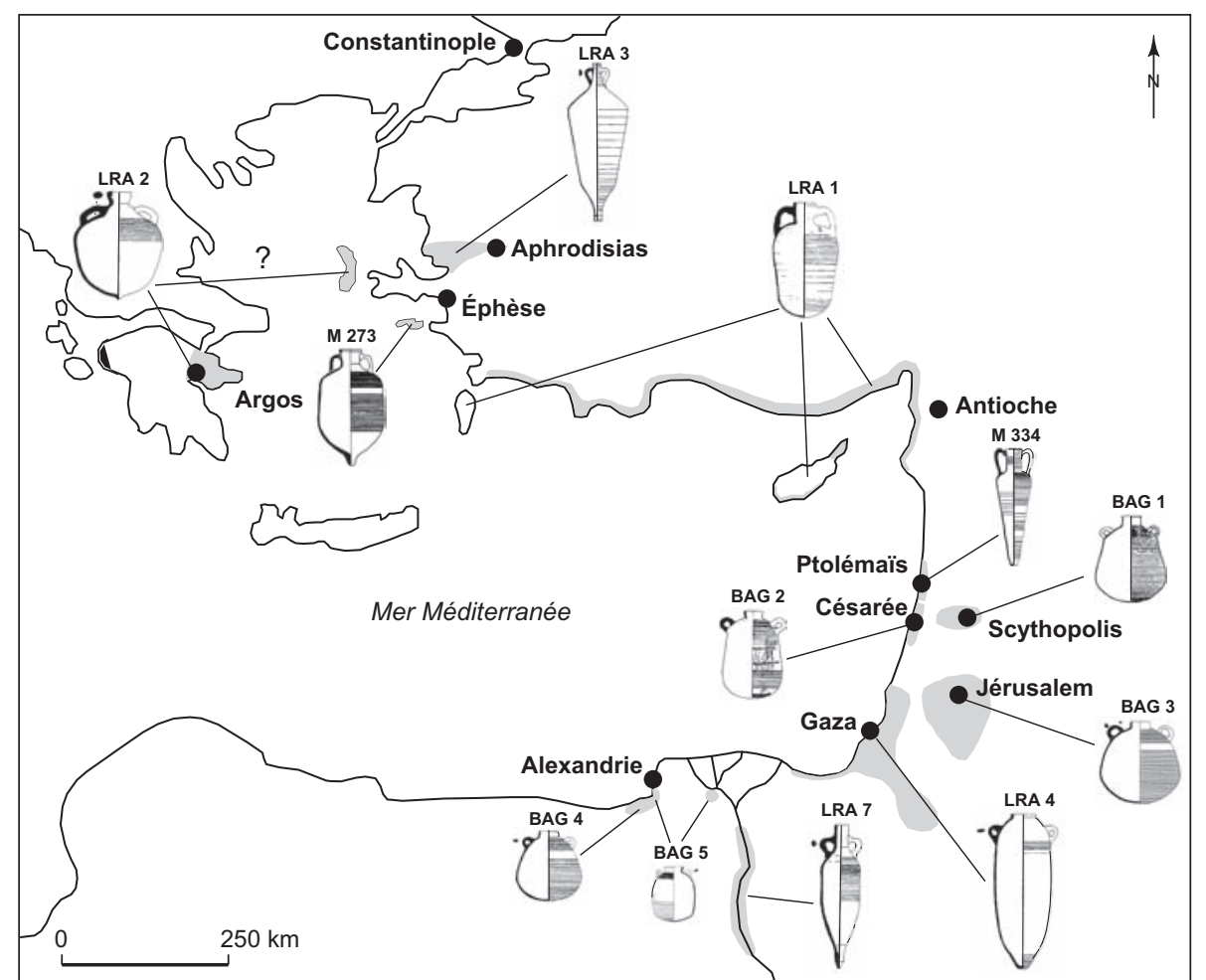

Fig. 82 - Carte des ateliers orientaux dont la diffusion est attestée en Gaule du Sud (DAO : D. Pieri, Université Paris-I). oriental, réputés non seulement pour leurs qualités hédoniques mais aussi pour leurs vertus curatives. Plusieurs recettes médicinales prescrites par d'éminents médecins de l'époque comme Alexandre de Tralles et Paul d'Égine relatent l'emploi de vins orientaux - en particulier les vins de Gaza et d'Ascalon - nécessaires dans le traitement d'affections diverses.

La présence et la diffusion en Gaule méridionale des emballages venus d'Orient semblent conditionnées par plusieurs facteurs.

Les importations orientales sont prédominantes en Gaule méridionale, attestées en particulier dans les zones d'arrivage des cargaisons le long du littoral méditerranéen. On remarque en effet une concentration très dense dans le Sud et plus particulièrement autour des sites portuaires du Sud-Est: on perçoit l'importance de Marseille dans cette diffusion qui essaime ces produits dans toute la basse vallée du Rhône, mais également, dans une moindre mesure, de Fos-sur-Mer et des sites portuaires secondaires de cabotage du Sud-Est. La pénétration dans les terres est concentrée dans la basse vallée du Rhône, conditionnée par la proximité des deux plus grands ports méditerranéens de Gaule, Marseille et Fos-sur-Mer. On en retrouve peu d'exemplaires en Gaule au-delà de Lyon, mais il est intéres- sant de souligner que ceux-ci sont principalement retrouvés le long des axes fluviaux et, en moindre quantité, le long du réseau routier terrestre. Les échanges commerciaux sur le territoire gaulois se font de préférence grâce aux cours d'eau, le réseau routier étant en partie délaissé du fait de sa dégradation, largement évoquée par les textes.

Le commerce du vin d'Orient paraît, avant tout, destiné aux consommateurs urbains. Le poids des villes dans la consommation est un fait incontestable. Cette disparité entre villes et campagnes est d'autant plus marquée au nord de la Gaule du fait d'une diffusion restreinte des emballages. Elle est limitée essentiellement aux cités de moyenne et de grande importance (Strasbourg, Nantes, Bordeaux, Paris, Tours).

Les principaux consommateurs appartiennent aux élites laïques et religieuses. Les quelques textes qui font mention de crus orientaux sont assez explicites sur ce point. Comme le montre Grégoire de Tours, seuls des personnages de haut rang social et fortunés, tels que le chambrier Eberulf ou cette femme de l'aristocratie lyonnaise qui faisait quotidiennement don d'un setier de vin de Gaza à la sacristie d'une basilique de la ville, pouvaient se permettre de tels achats. Les meilleurs vins de l'époque sont orientaux, comme le souligne Venance Fortunat, et ils sont indispensables à l'art 
de la table, appréciés par les élites locales jusqu'aux cours royales et impériales.

En France, les découvertes d'amphores à l'intérieur des terres sont loin d'illustrer l'ensemble des types importés, contrairement aux sites côtiers. Seules deux formes semblent bien diffusées: les LRA 1 de Cilicie et de Chypre et les $L R A 4$ de Gaza, mais cette diffusion semble avoir une valeur différente. Les LRA 1 étant des amphores qui «inondent» les marchés à cette époque, il est logique de les retrouver ailleurs que dans les ports, parfois même dans des endroits excessivement reculés comme à Castellu dans la haute montagne corse ou sur la petite île de Lavret au large des Côtes-d'Armor. En revanche, la présence et la diffusion en Gaule des LRA 4 et des bag-amphoras sont vraisemblablement sous-tendues par d'autres intentions. Ce sont des amphores qui ne réalisent pas un score d'enre-gistrement statistique suffisant pour que l'on soit en droit d'attendre de les voir diffusées si loin dans les terres, comme c'est le cas des $L R A 1$. Il est peut-être intéressant de relever qu'à distance des zones d'arrivage, lorsqu'elles sont retrouvées, les $L R A 4$ sont majoritaires sur les sites à vocation religieuse, comme à Ménerbes, Viviers, Digne-les-Bains, Lyon, Sion, Genève, Tours et Bordeaux qui, à de rares exceptions près, constituent pour l'instant les seuls lieux de découverte de formes LRA 4 en Gaule intérieure.

De plus, des textes de Grégoire de Tours et de Jean l'Aumônier indiquent clairement l'utilisation liturgique du vin de Gaza. Il n'est pas non plus à exclure que ce vin, très prisé, ait eu une connotation symbolique auprès des riches particuliers, suite à la promotion certainement faite par les nombreux pèlerins revenus de Terre sainte. Les bag-amphoras type 1, originaires de la région de Ptolémaïs (Saint-Jean-d'Acre), ont vraisemblablement elles aussi transporté un vin particulier. La présence systématique sur leur panse d'un décor peint stylisé, avec parfois une figuration de menorah, permet de supposer que ces emballages conditionnaient du vin peut-être destiné aux communautés juives, abondamment attestées dans les écrits des auteurs anciens.

En Occident, l'activité commerciale issue du grand commerce échappe en grande partie aux Occidentaux et ce sont des étrangers, surtout orientaux, qui s'en chargent désormais. Ils sont Grecs, Égyptiens, Juifs mais ce sont surtout les Syrii, terme général qui désigne des commerçants syriens, palestiniens et phéniciens, qui se spécialisent dans le grand commerce maritime. Ils sillonnent la Méditerranée et organisent des communautés dans les principaux centres en relation avec ce commerce méditerranéen (Rome, Ravenne,
Naples, Marseille, Arles, Carthage, etc.). Le cas de la Gaule en est une illustration parfaite : on peut retenir les exemples de Marseille, Arles, Narbonne, Toulouse, Bordeaux, Lyon, Tours, Nantes, Paris mais aussi Trèves et Cologne qui établissent un lien direct entre les découvertes de conteneurs orientaux et les attestations d'Orientaux mentionnées dans les textes. Les transmarini negociatores orientaux apparaissent donc bien comme des vecteurs essentiels de la diffusion des produits de luxe en Occident à l'époque romaine tardive et au haut Moyen Âge, produits qui sont vraisemblablement connus et recherchés grâce aux communautés originaires d'Orient.

Dans le courant du VIII ${ }^{\mathrm{e}}$ s., nous perdons la trace en Occident de ces conteneurs orientaux. Avec l'avènement du pouvoir carolingien, on assiste à un délaissement progressif de la Méditerranée au profit d'un nouvel axe économique plus septentrional. Le cœur des échanges se déplace vers l'Angleterre et la Scandinavie. En Orient même, la vitalité des grands centres producteurs s'effondre, d'une part par la perte des débouchés occidentaux, d'autre part par la promulgation de l'interdiction de consommer du vin et le durcissement des mesures antichrétiennes par la dynastie abbasside. On retrouve néanmoins sur place quelques exemples du maintien de productions héritées de la tradition céramique byzantine.

D. P.

\section{NOTICE 2}

\section{AMPHORES TARDIVES DANS LE SUD-OUEST DE LA GAULE}

Bordeaux et Toulouse ont commercé avec l'Afrique dès l'émergence de cette province dans le concert économique transméditerranéen (on a retrouvé, respectivement, 6 et 7 individus africains du $\operatorname{III}^{\mathrm{e}} \mathrm{s}$. dans ces deux villes). Bordeaux montre également quelques relations avec l'Orient à la même époque (3 Kapitän II).

Mais c'est surtout à partir du IV ${ }^{\mathrm{e}} \mathrm{s}$. que le développement du nombre des amphores tardives est important. Toulouse présente 61 individus originaires de la péninsule Ibérique et 38 individus africains ; Bordeaux, seulement 10 ibériques, mais 81 africains. Tous les types sont alors représentés: Africaine I et II, Africaines de moyenne dimension.

À Toulouse, au $\mathrm{V}^{\mathrm{e}}$ s., les importations lusitaniennes progressent (70 individus recensés), alors que les vais- 
seaux africains se raréfient (NMI : 16). En revanche, à la même époque, Bordeaux boude les produits ibériques (7 individus), au profit des amphores africaines (35). Les produits orientaux (LRA la et LRA 4a) sont présents dans les deux cités (12 à Toulouse, 10 à Bordeaux).

L'absence de niveaux importants $d u \mathrm{VI}^{\mathrm{e}} \mathrm{s}$. à Toulouse ne nous permet plus de fournir d'indications significatives sur le nombre et la répartition des amphores tardives dans cette ville. En ce qui concerne Bordeaux, le nombre des amphores africaines fléchit (NMI : 4), alors que les fabrications orientales (LRA 1b, LRA 2, LRA 3, LRA 4b, $L R A 5 / 6)$ connaissent un développement important (NMI : 24) tout au long de ce siècle et même au début du suivant. On notera toutefois que les produits africains arrivent, eux aussi, jusqu'à la fin du VI ${ }^{\mathrm{e}}$ s. et au début du VII ${ }^{\mathrm{e}}$ s. (Keay 62 et Keay 61).

En zone rurale, qu'il s'agisse des agglomérations ou des villae, il est impossible d'établir des statistiques : le nombre des amphores est trop peu important et il est seulement possible de relever la présence ou l'absence de ces amphores tardives. On constate alors que les chefs-lieux des cités plus modestes cessent assez tôt de recevoir ces vaisseaux lointains. Le problème se présente différemment pour les villae. Alors que certaines n'offrent rapidement plus d'amphores au-delà du $\mathrm{V}^{\mathrm{e}} \mathrm{s}$., d'autres, au contraire, possèdent dans leur sous-sol des types $\mathrm{du} \mathrm{VI}^{\mathrm{e}} \mathrm{s}$.

Ces constatations amènent plusieurs remarques. Tout d'abord, on aurait pu penser que la vague des envahisseurs barbares qui franchit le Rhin dans la nuit du 31 décembre 406 au $1^{\text {er }}$ janvier 407, et qui sema la désolation dans toute la Gaule jusqu'en Aquitaine, avant de passer pour partie en Espagne, avait mis fin à l'économie antique fondée sur le commerce à grande distance des surplus produits par les grands domaines. En fait, il n'en fut rien et, une fois la vague passée et les Wisigoths installés par un fodus en 418 , les pratiques commerciales antérieures reprirent leur cours.

On relève, ensuite, que tout se passe comme si, dès la fin du $\mathrm{V}^{\mathrm{e}}$ s., les capitales des cités moins importantes ne possédaient plus d'élites suffisamment fortunées pour importer des productions lointaines. On notera, à ce propos, que lorsque Grégoire de Tours évoque le vin de Gaza (qui a été conditionné dans les amphores de type LRA 4) ou le vin de Laodicée (conditionné, peut-être, dans des amphores de type $L R A 1$ ), c'est toujours une élite qui le consomme : l'évêque de Lyon, comme vin de messe, ou Claude et Eberulf, des familiers du roi. La même constatation est valable pour certaines grandes villae. L'archéologie montre bien que celles-ci ne sont pas abandonnées et qu'une activité persiste, mais la fonction résidentielle pour l'élite a disparu (les piscines sont remblayées ou utilisées à d'autres fins, par exemple). Il ne s'agit plus que d'un domaine administré par un régisseur pour le compte d'un grand propriétaire, mais celui-ci ne réside plus à cet endroit. La concentration des domaines en peu de mains, comme nous le laissent voir les textes contemporains, fait que les honestiores ne séjournent que dans quelques lieux et ne demeurent plus dans les autres.

Enfin, l'observation de l'origine des amphores tardives d'Aquitaine montre que les lieux d'approvisionnement diffèrent entre Toulouse et Bordeaux. Alors que Toulouse privilégie ses relations avec la Lusitanie et commerce peu avec l'Afrique, Bordeaux, au contraire, se tourne en priorité vers la Byzacène et délaisse la péninsule Ibérique. Et si l'on cherche des points de comparaison possibles, on constate que Toulouse présente un faciès identique à celui de Narbonne, alors que Bordeaux est à rapprocher de Marseille ou de Toulon, sans que l'on sache, au reste, pourquoi. Cela montre, cependant, l'importance de la route maritime par le détroit de Gibraltar; route qui acheminait les produits lusitaniens à Toulouse par Narbonne, alors que les amphores africaines et orientales empruntaient le chemin inverse pour gagner Bordeaux, et même toute la façade atlantique, puisque l'on retrouve des amphores tardives en grand nombre jusqu'en GrandeBretagne.

F. B.

\section{NOTICE 3}

\section{LES IMPORTATIONS D'AMPHORES À LYON PENDANT L'ANTIQUITÉ TARDIVE}

La position excentrée de Lugdunum par rapport au Bassin méditerranéen laissait présumer la précarité de son commerce extérieur au cours de l'Antiquité tardive. De surcroît, la perte de son statut politique au III $^{\mathrm{e}} \mathrm{s}$. suggérait l'anémie d'une cité qui se regroupait alors autour du quartier épiscopal, en rive droite de la Saône. Cependant, le développement des opérations d'archéologie préventive, à partir des années 1980, dans le quartier Saint-Jean, centre historique de Lyon, a fourni l'occasion de constituer des ensembles dont l'étude apporte des éléments déterminants sur la connaissance des relations commerciales 
de Lyon au cours de l'Antiquité tardive. L'exploitation $\mathrm{du}$ mobilier issu de la fouille de l'avenue Adolphe-Max a permis de constater l'existence et la diversité des exemplaires des amphores importées des régions méditerranéennes (Villedieu, 1985 et 1990 ; Bonifay, Villedieu, 1989). Depuis, les interventions archéologiques de l'îlot Tramassac et de l'îlot Clément-V opérées dans ce secteur du vieux Lyon ont livré leurs lots d'informations qui accréditent l'ouverture de la ville aux influences extérieures (Ayala, 1994 et 1998). Les résultats de ces études ont été largement enrichis par le développement des connaissances sur les amphores tardives à Lyon, portant sur l'identification des types en circulation, la détermination de leurs origines et de leur(s) contenu(s). De fait, le volume du matériel amphorique non identifié a décru en 20 ans passant de $40 \%$ à seulement $10 \%$. Ces progrès permettent d'établir le panorama des importations méditerranéennes et de vérifier le maintien des relations de Lyon avec les principales régions exportatrices de l'Empire. La récente fouille du parc Saint-Georges, réalisée sur la place Benoît-Crépu, a mis au jour de nombreux gisements amphoriques qui apportent par conséquent des informations supplémentaires sur la commercialisation des denrées du III ${ }^{\mathrm{e}} \mathrm{s}$. au $\mathrm{VI}^{\mathrm{e}} \mathrm{s}$. La présence à proximité d'un des ports de Lugdunum et l'utilisation de la berge comme dépotoir expliquent ce potentiel amphorique.

Les amphores à vin de Gaule Narbonnaise, principalement de type Gauloise 4, sont encore en circulation au milieu du $\mathrm{IV}^{\mathrm{e}} \mathrm{s}$. La fin de leur production semble être plus tardive.

Dans la péninsule Ibérique, les amphores à huile de Bétique Dressel 20 sont progressivement remplacées par les modèles tardifs Dressel 23 à partir de la seconde moitié du III ${ }^{\mathrm{e}}$ s., dont les importations perdurent jusqu'au début $\mathrm{du} \mathrm{V}^{\mathrm{e}} \mathrm{s}$. Les sauces de poisson proviennent principalement de Lusitanie à partir de la fin du $\mathrm{III}^{\mathrm{e}} \mathrm{s}$. dans les types Almagro 51C et également à partir du milieu du IV ${ }^{\mathrm{e}}$ s. dans les Almagro 5lA/B. Certaines d'entre elles pourraient être originaires des côtes andalouses. Elles sont encore présentes dans des contextes du début du $\mathrm{V}^{\mathrm{e}} \mathrm{s}$. Quant au vin de Bétique, réputé d'après les textes antiques, il a été probablement transporté en partie par les amphores Matagallares I, récemment recensées dans la région lyonnaise. Ces conteneurs abondamment attestés au $\operatorname{III}^{\mathrm{e}} \mathrm{s}$. et au début du siècle suivant n'apparaissent plus dans les ensembles postérieurs. Les denrées d'origine hispanique sont progressivement éclipsées à partir du milieu du $\mathrm{III}^{\mathrm{e}} \mathrm{s}$. par les produits nord-africains.
La pénétration des amphores africaines dans le marché lyonnais était jusqu'ici considérée comme timide au III ${ }^{\mathrm{e}} \mathrm{s}$, pour devenir importante seulement au siècle suivant. Or les données du parc Saint-Georges ont montré que leur indice de fréquence est très important dès le milieu du $\mathrm{III}^{\mathrm{e}} \mathrm{s}$. et ce, jusqu'au début du $\mathrm{V}^{\mathrm{e}} \mathrm{s}$. Les types identifiés permettent de percevoir des courants d'échanges privilégiés avec, tout d'abord, la province de Proconsulaire et, en second lieu, avec celles de Tripolitaine et de Maurétanie Césarienne. À partir du début du III ${ }^{\mathrm{e}}$ s., l'huile africaine est commercialisée dans les amphores Africaine I et Tripolitaine III qui disparaissent au début du siècle suivant. Les Africaine IIA, IIB et IIC1, transportant des saumures ou du vin, sont abondantes au milieu du III ${ }^{\mathrm{e}} \mathrm{s}$, pour être progressivement remplacées par les modèles Africaine IID à partir de la seconde moitié de ce siècle. Les variantes tardives Africaine IIC2 apparaissent dans les ensembles du milieu du $\mathrm{IV}^{\mathrm{e}} \mathrm{s}$. simultanément avec les types Keay 27A. Au début du IV s., les amphores de dimensions moyennes type Keay 25 sont commercialisées à Lyon jusqu'au siècle suivant avec différentes variantes. Les conteneurs africains cylindriques de petites dimensions Keay 26, ou spatheion, semblent apparaître dès la fin $\mathrm{du} \mathrm{IV}^{\mathrm{e}} \mathrm{s}$. et sont attestées avec quelques amphores cylindriques de grandes dimensions jusqu'au $\mathrm{VI}^{\mathrm{e}} \mathrm{s}$. Les conteneurs vinaires de Maurétanie Césarienne Dressel 30 sont progressivement remplacés par les types Keay lB à partir de la fin du $\operatorname{III}^{\mathrm{e}} \mathrm{s}$. Ces derniers perdurent jusqu'au milieu du IV ${ }^{\mathrm{e}}$ s. Par ailleurs, même si leur nombre est inférieur aux exemplaires italiques, quelques conteneurs vinaires Agora M254 d'origine africaine sont importés du $\mathrm{III}^{\mathrm{e}} \mathrm{s}$. jusqu'au milieu du IV ${ }^{\mathrm{e}} \mathrm{s}$.

Les importations d'amphores de Méditerranée orientale présentent une grande variété de productions. Dans les contextes du $\mathrm{III}^{\mathrm{e}} \mathrm{s}$. et du début du $\mathrm{IV}^{\mathrm{e}} \mathrm{s}$., des types récemment déterminés ou rares à Lyon sont attestés : Agora G199, Cnossos 18, Célestins 1A et Bitronconique égyptienne. Leur nombre reste tout de même faible par rapport à celui des conteneurs vinaires fréquents Kapitän I et II, Agora F65/66 et Rhodienne. Leur taux de fréquence devient par la suite totalement marginal. Au début du $\mathrm{V}^{\mathrm{e}} \mathrm{s}$., de nouveaux produits apparaissent sur le marché lyonnais. Il s'agit des Late Roman Amphora 1, 3 et 4. Les données récentes n'apportent pas d'informations supplémentaires sur ces conteneurs.

Enfin, les amphores italiques restent certes minoritaires, mais plusieurs types ont été identifiés. Les Agora M254, transportant probablement du vin de Sicile, sont commer- 
cialisées du $\mathrm{III}^{\mathrm{e}} \mathrm{s}$. jusqu'au milieu du $\mathrm{IV}^{\mathrm{e}} \mathrm{s}$. Au début du siècle suivant, les conteneurs vinaires Keay 52, dont l'origine est située en Calabre, sont introduits à Lyon. Quelques amphores à alun des îles Lipari, abondantes au III ${ }^{\mathrm{e}}$ s., sont encore attestées au début du IV ${ }^{\mathrm{e}} \mathrm{s}$.

Ces recherches ont permis de déceler que les dernières empreintes de la culture matérielle antique, au sein de laquelle les champs d'influence nord-africaine et orientale restent prédominants, se poursuivent jusqu'au $\mathrm{VI}^{\mathrm{e}} \mathrm{s}$., à l'aube de l'établissement du royaume burgonde. Quelques traces d'importations méditerranéennes sont visibles dans certains ensembles du VII ${ }^{\mathrm{e}}$ s., mais cela reste du domaine de l'anecdotique.

T. S.

\section{NOTICE 4}

\section{LES AMPHORES À ARLES AU IV'S.}

Depuis les années 1980, les fouilles menées dans différents quartiers de la ville d'Arles correspondaient en grande partie à des contextes de l'Antiquité tardive, avec une fourchette de datation allant de l'extrême fin du IV ${ }^{\mathrm{e}} \mathrm{s}$. à la fin du $\mathrm{VI}^{\mathrm{e}} \mathrm{s}$. Comme on a pu l'observer depuis quelques années, les niveaux du $\mathrm{IV}^{\mathrm{e}} \mathrm{s}$., jusqu'à maintenant, étaient pratiquement inexistants. Si on maîtrisait bien les importations d'amphores pour les contextes plus tardifs, on avait en revanche de la peine à imaginer les différents échanges commerciaux dans le courant $d u I^{e} \mathbf{s}$. Les fouilles menées sur le site de l'Esplanade en 2000 ont, pour la première fois, donné l'occasion de dégager des contextes d'occupation du milieu et du troisième quart $d u I^{\mathrm{e}} \mathrm{s}$., avec un mobilier céramique et amphorique très abondant. Les premières observations sur les amphores sont les suivantes :

- les amphores vinaires de Gaule Narbonnaise sont toujours très bien représentées au milieu du $\mathrm{IV}^{\mathrm{e}} \mathrm{s}$. et dans le troisième quart $\mathrm{du} \mathrm{IV}^{\mathrm{e}} \mathrm{s}$. On pourrait situer la fin de leur production et de leur diffusion vers la fin $d u \mathrm{IV}^{\mathrm{e}} \mathrm{s}$. ;

- les amphores à huile de Bétique Dressel 23B et 23C, peu répandues dans des contextes de la fin du $\mathrm{III}^{\mathrm{e}}$ s., sont en revanche très bien attestées au milieu du $\mathrm{IV}^{\mathrm{e}} \mathrm{s}$. Leur production et leur diffusion s'étendra jusqu'au premier tiers du $\mathrm{V}^{\mathrm{e}} \mathrm{s}$. ;

- les amphores Almagro 51A/B (22 ex.) et Almagro 51C (13 ex.), servant au transport des sauces de poisson, sont aussi très bien attestées au milieu du $\mathrm{IV}^{\mathrm{e}} \mathrm{s}$. Les différents types de pâte laissent supposer plusieurs lieux de production. Pour certains de ces containers ce serait la Bétique et pour d'autre la Lusitanie ;

- le commerce du vin de Bétique est attesté par les dernières diffusions d'amphores Matagallares 1 représentées seulement par trois exemplaires;

- les amphores italiques sont assez bien attestées au milieu et dans la seconde moitié du IV ${ }^{\mathrm{e}} \mathrm{s}$. Conçues pour le transport du vin, elles sont représentées par des amphores de petits modules de type Keay 52 en provenance de Calabre (9 ex.) et de type Agora M254 en provenance de Sicile (27 ex.). Ces dernières, majoritaires, ont une pâte de couleur rouge avec de nombreuses inclusions noires et de microfossiles qui rappellent les pâtes de Campanie. D'autres ont une pâte fine, plus claire, allant du rouge brique au rouge orangé, que l'on pourrait facilement identifier à une pâte africaine. La surface de ces amphores est souvent de couleur blanchâtre à jaune ou gris clair. Elles seront diffusées jusque dans la première décennie $d u \mathrm{~V}^{\mathrm{e}} \mathrm{s}$. ;

- au milieu du $\mathrm{IV}^{\mathrm{e}} \mathrm{s}$., les amphores africaines sont abondantes et dominent toutes les autres catégories. Servant au transport des saumures et de l'huile mais également du vin, elles sont représentées par 146 exemplaires. Les Keay 25 sont majoritaires avec 130 individus attestés essentiellement par les sous-types 1 (66 ex.) et 3 (64 ex.). Les Keay 25 sous-type 2 , absentes au milieu du IV ${ }^{\mathrm{e}}$ s. et dans le troisième quart $\mathrm{du} \mathrm{IV}^{\mathrm{e}} \mathrm{s}$., sont présentes dans des contextes datés du quatrième quart du $\mathrm{IV}^{\mathrm{e}} \mathrm{s}$. et dans le premier tiers du $\mathrm{V}^{\mathrm{e}}$ s. ;

- le type Keay 27 est attesté seulement par 4 exemplaires ainsi que les dernières productions africaines IIC et IID avec 3 individus ;

- les amphores vinaires Dressel 30/Keay 1B, encore bien présentes au milieu du $\mathrm{IV}^{\mathrm{e}} \mathrm{s}$. et dans la seconde moitié du $\mathrm{IV}^{\mathrm{e}} \mathrm{s}$., sont caractérisées par une pâte de couleur orange clair et surface jaunâtre. Le bandeau du col est légèrement cannelé. Pour ce type de conteneurs, jopterais plutôt pour des productions en provenance de Tunisie que de Mauritanie Césarienne (Algérie) ;

- enfin, les amphores orientales restent minoritaires au milieu du $\mathrm{IV}^{\mathrm{e}} \mathrm{s}$. et dans le troisième quart du $\mathrm{IV}^{\mathrm{e}} \mathrm{s}$. Seul le type $L R A 3$ est attesté avec 8 individus.

Si l'étude du mobilier céramique du site de l'Esplanade est loin d'être terminée, nous pouvons déjà réfléchir sur les différents échanges commerciaux de cette période, méconnus jusqu'à maintenant.

J. P. 


\section{NOTICE 5}

\section{LES FACIÈS CÉRAMIQUES DU VII' S. À MARSEILLE}

Les travaux récents menés sur les contextes du VII ${ }^{\mathrm{e}} \mathrm{s}$. marseillais ont permis de mieux cerner l'évolution chronologique d'un répertoire de céramiques dont le classement avait déjà été abordé dans la synthèse du matériel tardif marseillais (faciès D et $\mathrm{E}$ : Bonifay et al. dir., 1998). Si le faciès $\mathrm{D}$ (extrême fin du VI $\mathrm{I}^{\mathrm{e}} \mathrm{s}$. et première moitié $\mathrm{du} \mathrm{VII}^{\mathrm{e}} \mathrm{s}$.) était alors relativement bien documenté, il n'en était pas de même du faciès E (seconde moitié du VII ${ }^{\mathrm{e}} \mathrm{s}$. et début du VIII ${ }^{\mathrm{e}}$ s.), représenté seulement par un très petit nombre de contextes (essentiellement ceux de la place Jules-Verne). Les nouveaux ensembles de mobilier des sites de l'Alcazar et du pavillon Bargemon que concernent ces études n'apportent que quelques compléments au faciès D (Bien, 2001, 2003, 2005a et b ; Waksman et al., 2003 et 2005). En revanche, ils permettent de renouveler complètement notre connaissance du faciès $\mathrm{E}$ et d'établir une première ébauche de typologie évolutive de ce matériel très tardif.

Les premiers contextes (phase 1: période 1a, 1b, Marseille-Alcazar), datés du premier tiers du $\mathrm{VII}^{\mathrm{e}} \mathrm{s}$. (faciès D), sont marqués par la présence de sigillées africaines D : Hayes 90B/105, 109A, 105, 107 et 108, 99C (variante au rebord atrophié), 91D, associées à des lampes de type Hayes IIB/Atlante $\mathrm{X}$ à décor surmoulé. La vaisselle culinaire qui compose ce premier ensemble est majoritairement d'origine orientale: groupe «atelier X » (Waksman et al., 2005), cruche égyptienne Egloff 227, marmite égéenne de type indéterminé. Elle concerne également quelques céramiques communes africaines (bol à listel, voir Fulford, Peacock, 1984, fig. 76, no 2) et italiques (marmites de type 13 : CATHMA, 1991). Les amphores africaines Keay 61C, Keay 61D et Keay 62 tardive apparaissent également dans ce premier groupe, aux côtés des amphores orientales Carthage $L R A 4 \mathrm{~B}, L R A 5 / 6$ auxquelles il faut très certainement ajouter les types $L R A 1 \mathrm{~B}$ et $2 \mathrm{~B}$, absents dans nos contextes.

Un deuxième ensemble légèrement plus tardif (phase 2 : période 1c, Marseille-Alcazar, et premier état du pavillon Bargemon) correspond à la fin du faciès D (autour du milieu du VII ${ }^{\mathrm{e}} \mathrm{s}$. ?). Il regroupe des coupelles de type Hayes 108 et proches du type Hayes 108 qui présentent un profil différent des précédentes. Marqué également par la présence du conteneur oriental Carthage LRA 1B soustype 2 , il contient des rebords de DS.P. et de céramique commune à pâte grise. Il regroupe également les coupelles égyptiennes Gempeler T344 (Gempeler, 1992) et africaines Hayes 80B/99, auxquelles est associée une variante du plat Hayes 109 possédant une paroi plus fine qui tendrait à se rapprocher de la variante B à paroi mince (Bonifay, 1998, p. 78) sans pour autant égaler sa finesse. Cette forme, que nous classons en variante $\mathrm{A} / \mathrm{B}$, pourrait correspondre à une production intermédiaire peut-être légèrement plus tardive que la forme Hayes 109A. Cet ensemble regroupe aussi la marmite égéenne de type Reynolds, 1995, pl. 57, nº 468.

Une troisième phase (période $1 b$, Marseille-Alcazar, second état du pavillon Bargemon) correspondant au faciès $\mathrm{E}$ est datée du troisième quart du $\mathrm{VII}^{\mathrm{e}} \mathrm{s}$. Elle est marquée par l'apparition des conteneurs africains Keay 50, Keay 61A et Keay 26 tardif à paroi fine qui sont notamment associés à des variantes tardives des amphores Keay 61 et Keay 62 dont des exemplaires con orlo a fascia sont jusqu'ici non répertoriés (type 52, 8 dans Bonifay, 2004). Les amphores orientales, présentes à travers les types Carthage $L R A 1 \mathrm{~B}, L R A 4 \mathrm{~B}$ et $L R A 4 \mathrm{C}$, regroupent également des variantes plus atypiques du conteneur LRA 5/6 qui comportent un rebord au profil en $\mathrm{S}$ plus ou moins épais et qui sont sans doute fabriquées en Égypte. Cette phase est également marquée par la présence d'une variante tardive de l'amphore d'Italie méridionale Keay 52 (Calabre) pour laquelle nous avons trouvé des éléments de comparaison dans les niveaux les plus tardifs de la Crypta Balbi à Rome (Saguì, 1998, fig. 11). La vaisselle de table d'origine africaine rencontrée concerne dans un premier temps un répertoire connu: types Hayes 99C, Hayes 105 et Hayes 109B. D'autres exemplaires tunisiens sont plus atypiques, certains d'entre eux sont ou paraissent semblables à des céramiques mises au jour sur le site de Sidi Jdidi (contexte 6 : Bonifay et al., 2004). Pour la vaisselle, il faut noter la présence d'une cruche orientale de type indéterminé mais surtout des marmites égéennes qui sont dominantes : type Reynolds, 1993, pl. 57, no 468 et pl. 65 , no 760 ; type Ricci, 1998, fig. 4, no 5 ; type Hayes, 1968, fig. 108. Des productions locales en céramiques communes à pâte grise et en DS.P. viennent compléter ce répertoire.

La phase suivante (période 2, Marseille-Alacazar) s'inscrit dans la continuité de la précédente. Quelques objets, dont les profils apparaissent sensiblement différents, montrent toutefois des signes d'évolution par rapport à ceux qui composaient la phase 3 : type Keay 26 tardif, amphore con orlo a fascia, variante tardive de conteneur $L R A 5 / 6$, des sigillées africaines Hayes 105 et Hayes 80B/99. On observe également la présence de la marmite de type Reynolds, 1993, pl. 65, no 760, une amphore classée en variante tardive 
Keay 61C ainsi que des conteneurs orientaux Carthage $L R A 1 C$ et $L R A 4 C$. Deux exemplaires d'amphores indéterminées leur sont associés. Quelques formes de céramiques communes à pâte grise ou égéennes atypiques viennent compléter ce groupe. Un follis en bronze de Constantin IV (668-685), frappé en Sicile, mis au jour dans cette phase, nous apporte par ailleurs un précieux terminus post quem.

La dernière phase (période 3, Marseille-Alcazar) qui compose ce faciès $\mathrm{E}$ reste à ce jour peu documentée. L'association d'un fond bombé en céramique commune à pâte grise, d'un exemplaire au rebord plus court que les précédents de la marmite de Saraçhane (type Hayes, 1968, fig. 108) - ce qui correspond sans doute à un signe d'évolution encore mal connu à ce jour - et d'une amphore globulaire, indique sans doute une chronologie plus tardive située dans le courant du VIII ${ }^{\mathrm{e}}$ s., peut-être vers le deuxième quart de ce siècle. Cette phase regroupe également un plat Hayes 105.

Loin d'être exhaustive, cette courte liste de matériel (à compléter par l'apport des nouvelles études en cours) apporte une quantité importante d'informations sur la dynamique de la cité phocéenne à la fin du VII ${ }^{\mathrm{e}} \mathrm{s}$. On perçoit nettement un changement dans la nature du mobilier au cours de cette période. Ainsi, les importations de céramiques culinaires levantines diminuent tandis qu'augmentent celles en provenance de Constantinople (Saraçhane : Hayes, 1968), des Cyclades ou d'Anatolie égéenne (Waksman et al., 2005). Il semble ainsi probable que pour cette partie du mobilier le commerce était plus orienté vers Constantinople et le monde égéen dans la seconde moitié du VII ${ }^{\mathrm{e}} \mathrm{s}$. qu'au début du siècle. On note en revanche une persistance des importations d'Afrique du Nord: les conteneurs africains semblent devenir plus fréquents que les amphores orientales tandis que la céramique sigillée constitue environ $13,2 \%$ de l'ensemble de la vaisselle. Un des faits marquants de ces premières études réside sans aucun doute dans la découverte de DS.P. produites assez tardivement au VII ${ }^{\mathrm{e}} \mathrm{s}$.

S. B.

\section{NOTICE 6}

\section{TYPOLOGIE DES CÉRAMIQUES COMMUNES MÉDITERRANÉENNES}

Dans cette notice, les numéros en gras renvoient à la figure 83 .

\section{CÉRAMIQUES COMMUNES AFRICAINES}

1. Céramique culinaire africaine. Prototype de la forme Cathma 3 (Fulford 69/19.1). Marseille, fouilles du tunnel de la Major. Premier tiers du V ${ }^{\mathrm{e}} \mathrm{s}$. Étude S. Bien, dessin G. Frommhertz.

2. Céramique culinaire africaine. Ultime variante de la cocotte Hayes 197 et couvercle Hayes 196. Arles, fouilles de l'IRPA. Fin IV ${ }^{\mathrm{e}} \mathrm{s}$--premier quart $\mathrm{du} \mathrm{V}^{\mathrm{e}} \mathrm{s}$. Étude J. Piton, dessin de l'auteur.

3. Céramique africaine à pâte orange et surfaces blanchâtres. Mortier. Marseille, fouilles de la Bourse (De Mitri, 2001, fig. 12, $\mathrm{n}^{\mathrm{o}} 1$ ). Première moitié du $\mathrm{V}^{\mathrm{e}} \mathrm{s}$.

4. Céramique africaine à pâte orange et surfaces blanchâtres. Mortier Cathma 10 (Fulford 76/2.4). Hyères, Olbia, îlot 6. Fin du V ${ }^{\mathrm{e}}$ s.-premier tiers du $\mathrm{VI}^{\mathrm{e}} \mathrm{s}$.

5. Céramique africaine à pâte orange et surfaces blanchâtres. Mortier type Fulford 77/11.3. Marseille, fouilles de la place Jules-Verne (Cœeur-Mezzoud, 1996, fig. 69, nos 324325). Fin du VI ${ }^{\mathrm{e}} \mathrm{s}$.

6. Céramique africaine à pâte orange et surfaces blanchâtres. Cruche à décor peint. Marseille, fouilles de la Bourse. Première moitié du $\mathrm{V}^{\mathrm{e}} \mathrm{s}$.

\section{CÉRAMIQUES COMMUNES DE MÉditerRANÉE ORIENTALE}

7. Céramique culinaire du Proche-Orient. Marmite à fond bombé. Marseille, fouilles de la Bourse (CourMezzoud, 1998, fig. 130, $\mathrm{n}^{\text {os }}$ 204-205). Deuxième quart du $\mathrm{V}^{\mathrm{e}} \mathrm{s}$.

8. Céramique culinaire du Proche-Orient (Waksman et al., 2003, fig. 3 et 2005). Production de Tell Keisan (Florimont, 1984, pl. 16, nos 1-5) ? Marmite à fond bombé Cathma 29. Type très fréquent à Beyrouth. Sète, quartier du Barrou. Seconde moitié du $\mathrm{V}^{\mathrm{e}} \mathrm{s}$.

9. Céramique culinaire du Proche-Orient. Cocotte et couvercle à bord coupé. La Ciotat, île Verte (Vasselin, 2003, fig. 13, no 4). Fin du VI ${ }^{\mathrm{e}}$ s.-début du VII ${ }^{\mathrm{e}} \mathrm{s}$.

10. Céramique culinaire de mer Égée. Pot à cuire Cathma 17. Marseille, fouille de la Bourse. Fin du IV ${ }^{\mathrm{e}}$ s.première moitié du $\mathrm{V}^{\mathrm{e}} \mathrm{s}$.

11. Céramique culinaire de mer Égée. Marmite Cathma 5. Port-Vendres, gisement sous-marin Port-Vendres I (Pasqualini, Tréglia, 2003, fig. 9, n 50). Seconde moitié du $\mathrm{V}^{\mathrm{e}}$ s.-début du $\mathrm{VI}^{\mathrm{e}} \mathrm{s}$.

12. Céramique culinaire de mer Égée. Marmite Cathma 5, variante tardive. Marseille, fouilles de la Bibliothèque muni- 

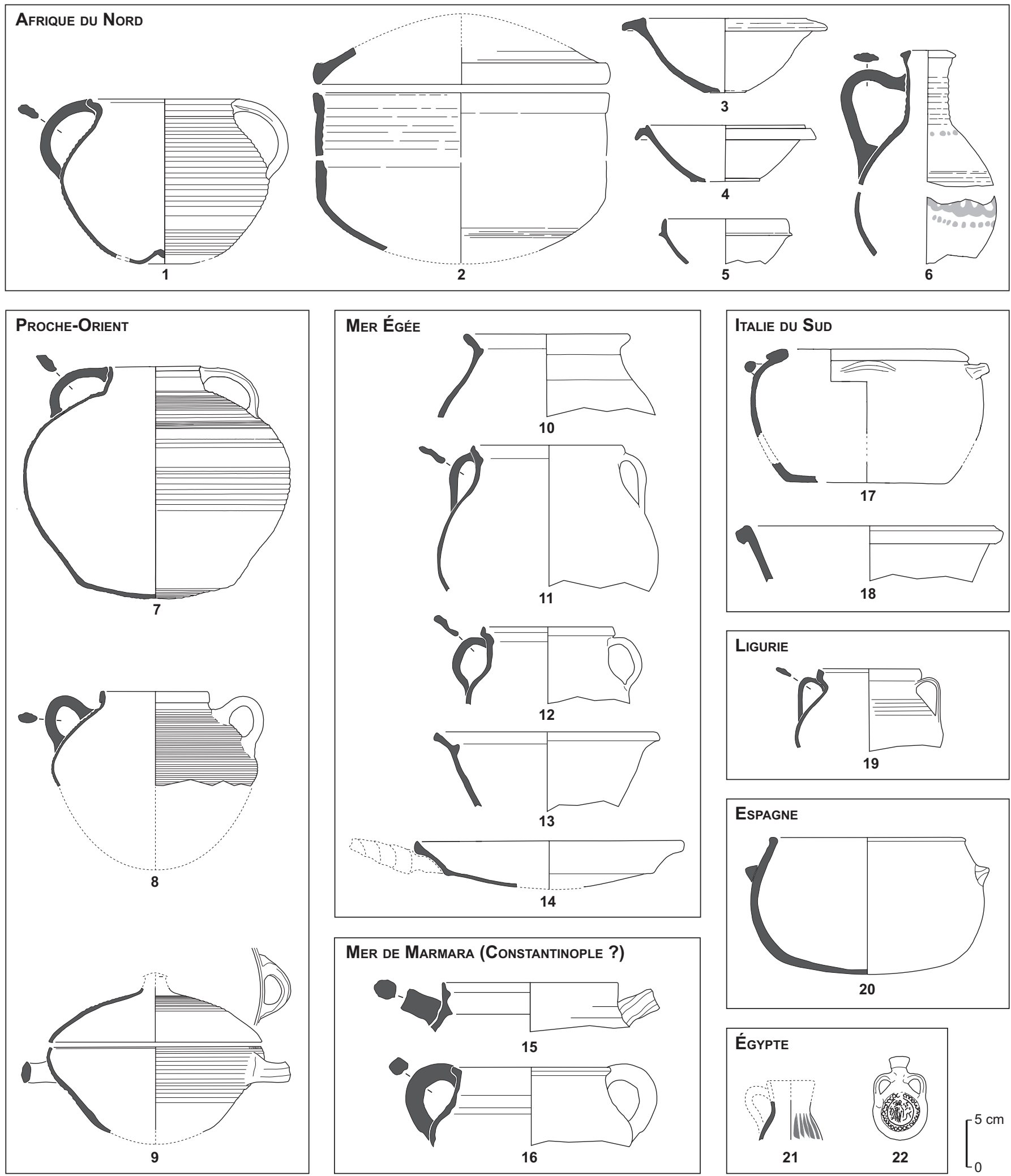

Mer de Marmara (Constantinople ?)
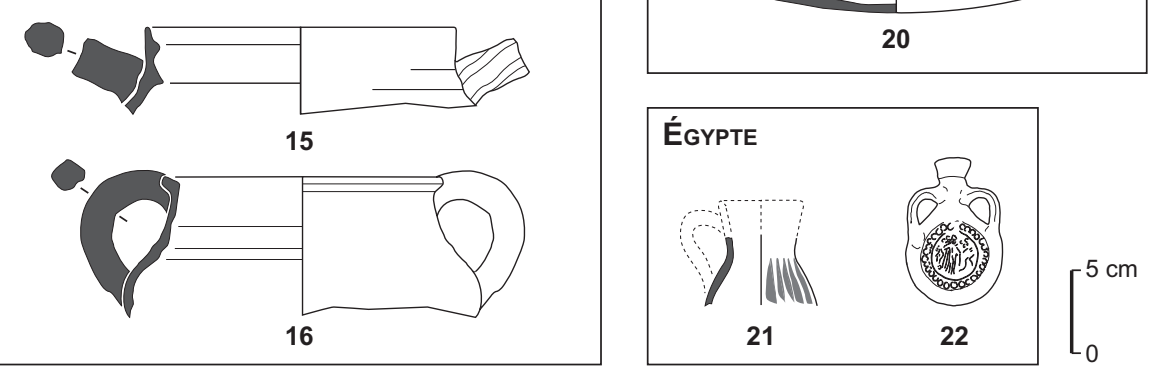

Fig. 83 - Sélection d'ustensiles culinaires importés en Gaule du Sud durant l'Antiquité tardive (DAO : J.-C. Tréglia, CNRS). 
cipale à vocation régionale (BMVR) Alcazar. Fin du VI ${ }^{\mathrm{e}}$ s.premier tiers du VII ${ }^{\mathrm{e}} \mathrm{s}$.

13. Céramique culinaire de mer Égée. Cocotte. PortVendres, gisement sous-marin Port-Vendres I (Pasqualini, Tréglia, 2003, fig. 10, $\mathrm{n}^{\mathrm{o}}$ 51). Fin du $\mathrm{IV}^{\mathrm{e}} \mathrm{s}$.-premier tiers du V $\mathrm{v}^{\mathrm{e}} \mathrm{s}$.

14. Céramique culinaire de mer Égée. Poêle à fond bombé Fulford 71/5.1. Hyères, Olbia, îlot 6. Premier tiers $\mathrm{du} \mathrm{VI}^{\mathrm{e}} \mathrm{s}$.

15. Céramique culinaire de la mer de Marmara. Marmite de Saraçhane (Istanbul), type Cathma 23. Marseille, fouilles de la BMVR Alcazar. Seconde moitié du VII ${ }^{\mathrm{e}} \mathrm{s}$.

16. Céramique culinaire de la mer de Marmara. Même groupe de production que le type Cathma 23. Marseille, fouilles de la BMVR Alcazar. Seconde moitié du VII ${ }^{\mathrm{e}} \mathrm{s}$.

\section{CÉRAMIQUES COMMUNES DES ÎLES DE MÉDITERRANÉE CENTRALE}

17. Céramique non-tournée. Production de la côte occidentale de la Sardaigne? Marmite Cathma 7 (Fulford 59/32.1). Toulon, fouilles du quartier Besagne (Bérato et al., 1986, fig. 34, $\mathrm{n}^{\mathrm{o}} 55$ ). Seconde moitié du $\mathrm{V}^{\mathrm{e}}$ s.premier tiers du $\mathrm{VI}^{\mathrm{e}} \mathrm{s}$.

18. Céramique culinaire sud-italique. Production de Pantelleria? (Italie). Cocotte carénée Carsana 121/1121

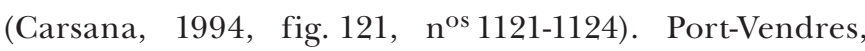
gisement sous-marin Port-Vendres I (Pasqualini, Tréglia, 2003, fig. 3, $\mathrm{n}^{\mathrm{o}} 16$ ). Fin du $\mathrm{IV}^{\mathrm{e}} \mathrm{s}$.-premier tiers du $\mathrm{V}^{\mathrm{e}} \mathrm{s}$.

19. Céramique culinaire ligure. Marmite Cathma 13. Fouille de Saint-Blaise (Vallauri, 1994, fig. 71, $\mathrm{n}^{\text {os }} 68-73$ ). Fin du VI ${ }^{\mathrm{e}}$ s.-premier tiers du VII ${ }^{\mathrm{e}} \mathrm{s}$.

\section{AUTRES CÉRAMIQUES COMMUNES}

20. Céramique non-tournée. Espagne méridionale? Hyères, Olbia, fouilles anciennes, hors stratigraphie. Ce type est très fréquent à Arles dans les contextes datés entre le milieu du $\mathrm{IV}^{\mathrm{e}} \mathrm{s}$. et le début du $\mathrm{V}^{\mathrm{e}} \mathrm{s}$.

21. Céramique à pâte calcaire égyptienne. Maréotide. Cruche Egloff 227-228. Marseille, fouilles de la BMVR Alcazar. Fin du $\mathrm{VI}^{\mathrm{e}}$ s.-premier tiers du $\mathrm{VII}^{\mathrm{e}} \mathrm{s}$.

22. Céramique à pâte calcaire égyptienne. Maréotide. Production des monastères d'Abu Mina? Ampoule à eulogie de saint Ménas. Paris, fouilles de la rue Dante en 1901 (Périn, 1985, p. 137, fig. 37). D’autres exemplaires sont attestés à Arles et à Marseille (BMVR Alcazar, ancien cimetière Saint-Charles).

J.-C. T.

\section{NOTICE 7}

\section{LES DÉRIVÉES-DES-SIGILLÉES PALÉOCHRÉTIENNES «MARSEILLAISES »}

La céramique DS.P. est un type de céramique fine dont plusieurs productions se trouvent dans la moitié sud de la Gaule. La production dite marseillaise est l'une des plus importantes (Rigoir J., 1968). Elle est largement diffusée dans le bassin nord-ouest de la Méditerranée, plus faiblement vers l'intérieur du pays où existent de nombreuses petites productions locales.

Aucun grand atelier de DS.P. n'est actuellement connu (seulement quelques petits ateliers ont été mis au jour à Générac, à la Quintarié et à Saint-Péray). L'argile utilisée pour les vaisselles des DS.P. marseillaises nous amène à penser que les ateliers se trouvaient dans la région de Marseille et d'Aubagne (Pelletier et al., 1995).

L'évolution typologique des DS.P. marseillaises, qui n'a pas connu de changements rapides, reste toujours une question délicate. Les études récentes arrivent peu à peu à dessiner ses grandes lignes (fig. 84).

Dans la première phase de production, qui commence vers la fin $\mathrm{du} \mathrm{IV}^{\mathrm{e}} \mathrm{s}$. et le début $d u \mathrm{~V}^{\mathrm{e}} \mathrm{s}$., il semble que les DS.P. reproduisent des formes proches de celles déjà fabriquées dans la production languedocienne, comme les formes Rigoir 6, 8 et 18b. C'est le cas également des décors qui présentent des combinaisons de poinçons d'une richesse comparable à celle des compositions languedociennes (Rigoir et al., 1985). Mais l'activité des ateliers marseillais se traduit très vite par une grande diversité de formes qui persiste jusqu'à la fin de la standardisation de la production.

C'est vers le deuxième quart $d u \mathrm{~V}^{\mathrm{e}} \mathrm{s}$. que la production commence à se standardiser et la période de grande diffusion se situe vers la seconde moitié de ce même siècle. Les formes les plus emblématiques de cette production standardisée sont l'assiette Rigoir 1 au marli quadrangulaire et épais, et le bol caréné Rigoir 18a portant un décor de guillochis sur le col et un décor estampé, souvent des arceaux Rigoir 199 ou 200, sur la panse. Le haut degré de standardisation de ces formes permet une identification morphologique très précise. 


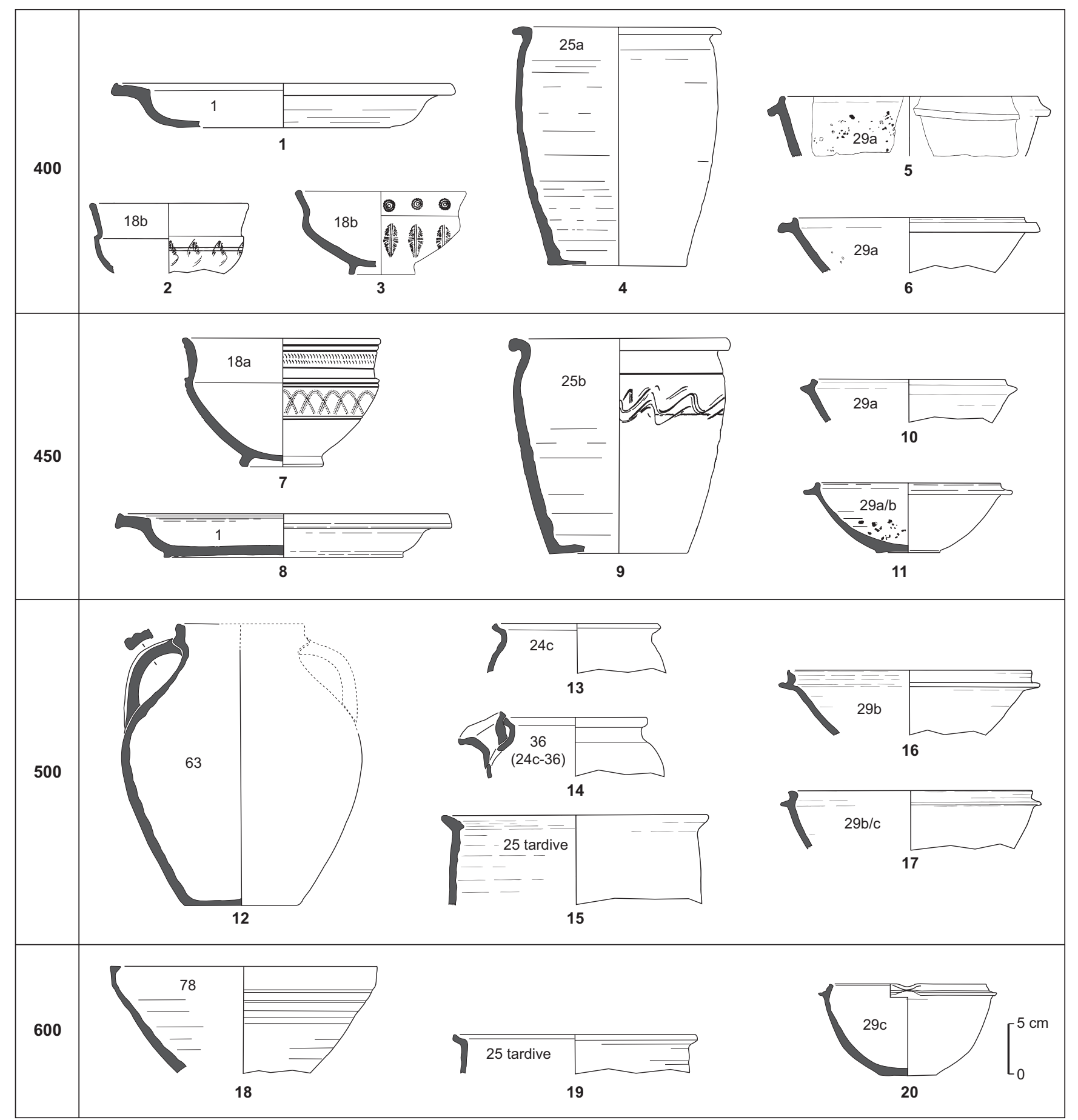

Fig. 84 - Évolution des Dérivées-des-Sigillées Paléochrétiennes « marseillaises » selon la typologie de J. et Y. Rigoir (DAO : T. Mukai). 
Des tentatives de classement en plusieurs groupes durant cette grande époque de production s'amorcent, d'une part, sur la base des analyses chimiques qui opposent deux groupes d'argiles (Pelletier et al., 1995, p. 115 et fig. 8) et, d'autre part, à la suite de l'examen attentif de la morphologie des vases et de la composition des poinçons utilisés pour les décorer (Mukai, 2001; Rigoir et al., 2001). Cette démarche montre qu'un ensemble d'ateliers employant les mêmes poinçons fabrique, jusqu'au début $\mathrm{du} \mathrm{VI}^{\mathrm{e}} \mathrm{s}$., les formes emblématiques de cette production standardisée.

La production connaît un grand changement au cours $\mathrm{du} \mathrm{VI}^{\mathrm{e}} \mathrm{s}$. À partir du deuxième quart de ce siècle, on remarque la diminution du répertoire des formes qui n'est représenté presque exclusivement que par des formes utilitaires, comme les formes Rigoir 24c, 24c-36, 25 tardive, $29 \mathrm{~b}$ et 63 , et la quasi-absence des décors et de l'engobe sur les vases (Bonifay, Pelletier, 1983). Ces formes fabriquées dans une pâte dont la qualité est devenue très irrégulière montrent une ressemblance de leurs profils avec ceux de la production de céramique commune grise.

La production des DS.P. marseillaises, proprement dite, se poursuit certainement jusqu'à la fin du VII ${ }^{\mathrm{e}} \mathrm{s}$. sans grand changement, les formes Rigoir 29c, 63 et 78 étant alors les plus typiques. Il est probable que la disparition des DS.P. ne découle que d'une assimilation avec la céramique commune grise à partir du VIII ${ }^{\mathrm{e}} \mathrm{s}$.

À partir de la seconde moitié du $\mathrm{VI}^{\mathrm{e}}$ s., il est évident que les DS.P. marseillaises ne sont plus fabriquées dans un établissement «industrialisé ». Dans ce cas, quelle différence existait-il entre les ateliers de DS.P. tardives et ceux de céramique commune grise ? Certes, au point de vue céramologique, on peut percevoir une différence entre les pâtes de ces deux types de céramique gauloise. Cependant, il n'existe guère de différence dans le produit pour les consommateurs.

T. M. 